\title{
SELECTION AND CHARACTERISATION OF SINGLE-STRANDED DNA APTAMERS FOR TRICLOSAN
}

A THESIS SUBMITTED TO VICTORIA UNIVERSITY OF WELLINGTON IN PARTIAL FULFILMENT OF THE REQUIREMENTS FOR THE DEGREE OF DOCTOR OF PHILOSOPHY

BY

SHIWEI LI

2016

TE WHARE WĀNANGA O TE ÜPOKO O TE IKA A MĀUI

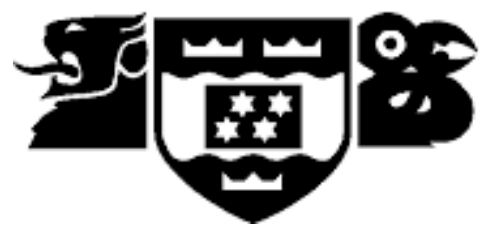


To my family 


\section{ACKNOWLEDGEMENTS}

I thank my primary supervisor Professor Kenneth McNatty for giving me the opportunity to undertake this research for my doctoral thesis in his laboratory. He was very patient and gave me thoughtful advice throughout this project. His advice was immensely useful. I also thank my secondary supervisors Dr Mark Clarkson and Associate Professor Douglas Eckery for their guidance, support and encouragement during the completion of this thesis and telling me always to believe in myself.

I thank the many past and present members of McNatty group for their aid in various aspects of the research process. In particular, I appreciate the patience and support from Adrian Bibby and Dr Shalen Kumar. Arian Bibby was irreplaceable in his assistance in the laboratory and his willingness to teach me techniques outside my discipline was invaluable. Dr Shalen Kumar was always willing to share his experience with me. His thoughtful advice helped determine how to perform aptamer selection experiments. In addition, I am deeply grateful for the many discussions and friendships cultivated during my tenure in the group, including Dr Janet Pitman, Dr Catherine Davis, Zaramasina Clark, and Derek Heath.

I wish to recognise the help from Dr Alan Clark, Dr Junsheng Lin and Dr Omar Alsager. They have always been there for me and helping take this research to a level that may not have otherwise been possible. Specifically, I thank Dr Clark for teaching me how to prepare affinity matrices. I thank Dr Alsager for showing me how to work with gold nanoparticles and sharing ideas from his experimental work. I thank Dr Lin who has guided me for the development of a novel imaging-based assay.

Above all, this thesis would have not been successful if not financially funded by my former employer, Callaghan Innovation (Wellington, New Zealand). I have enjoyed working there for five years, and I deeply appreciate the support and professional training provided by Dr Diana Siew, Dr Mark Clarkson and Dr Joseph Deere. Finally, I have relied on the support of my family to achieve all successes throughout my life. To my family I owe more than to anyone else. They have guided me, encouraged me, and sustained me. Most of all, they have always believed in me and loved me. All of this means more to me than I am able to express here in words. 


\section{TABLE OF CONTENTS}

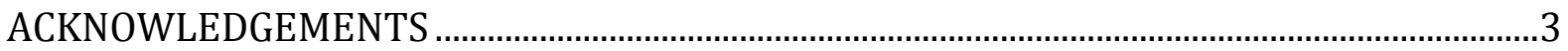

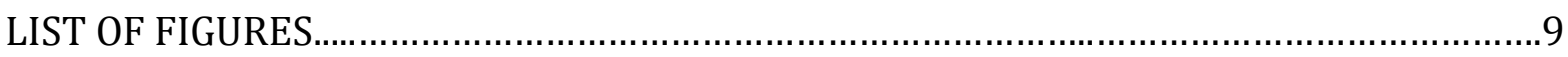

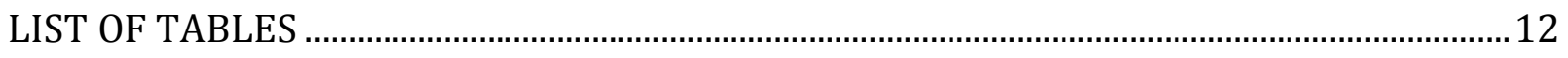

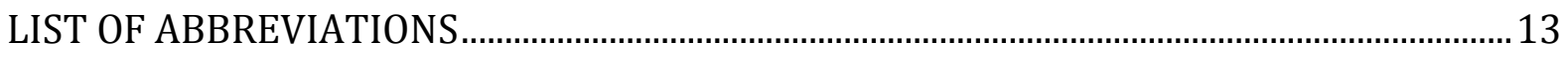

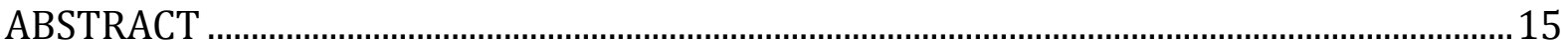

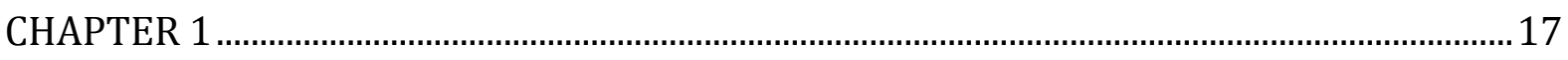

GENERAL INTRODUCTION ..............................................................................................

1.1 Endocrine-disrupting compounds (EDCs) …...................................................................17

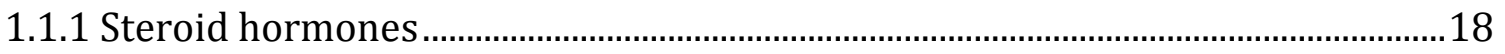

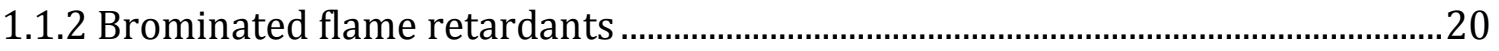

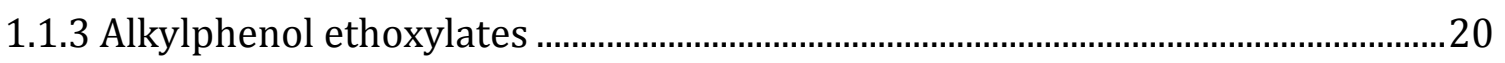

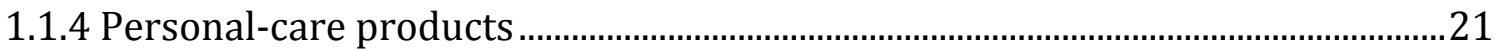

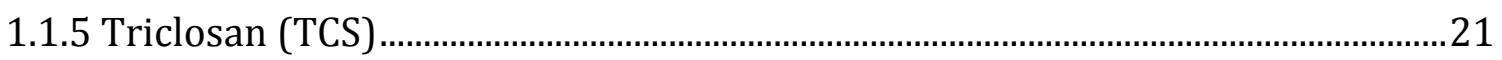

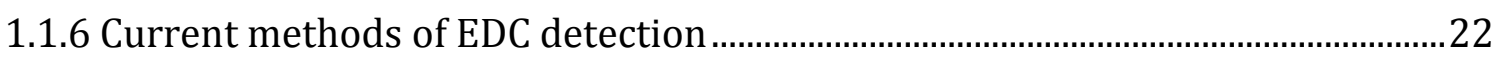

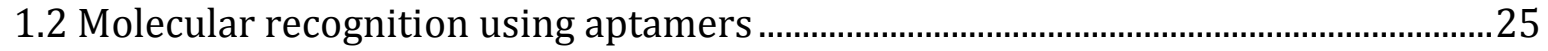

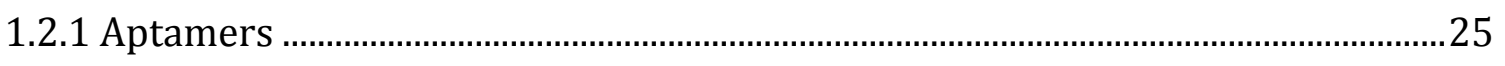

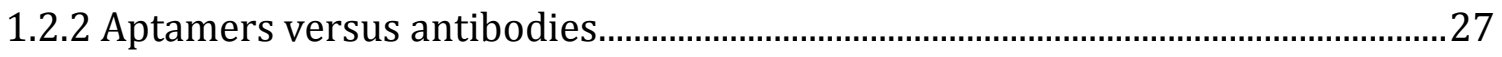

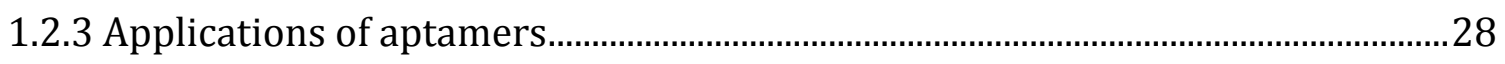

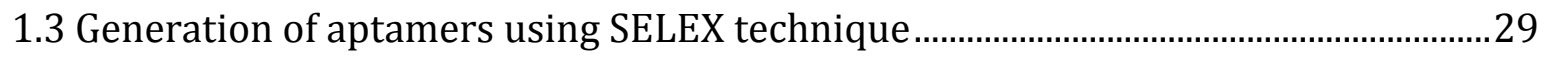

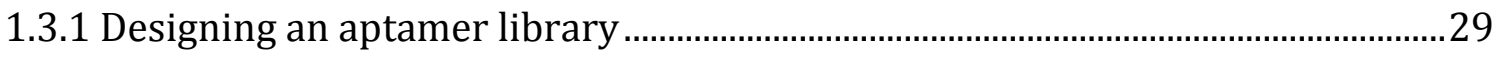

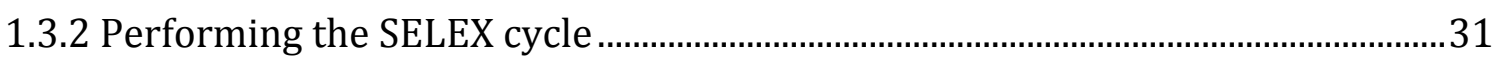

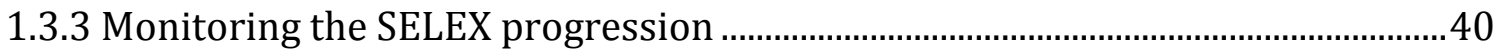

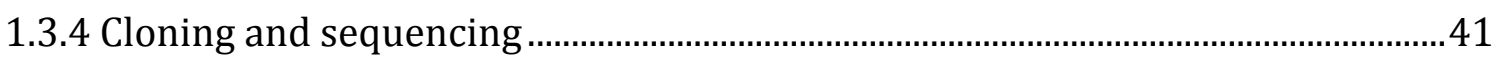

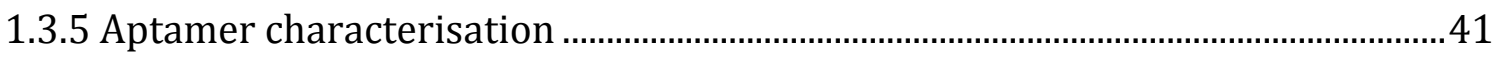

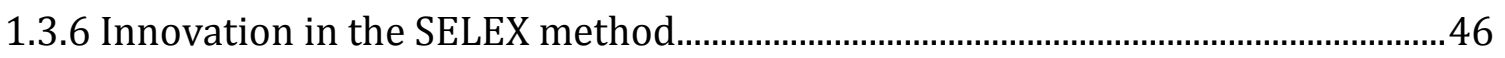

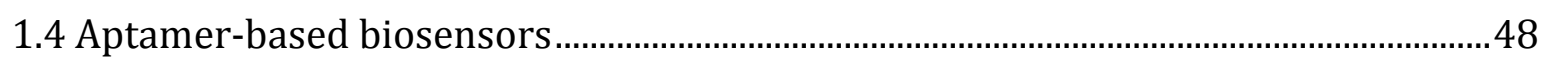

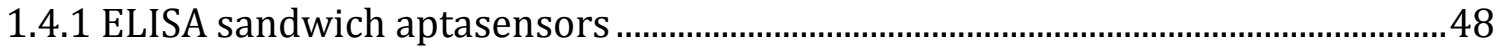

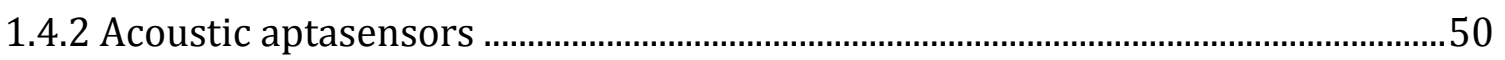

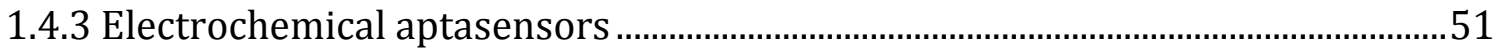

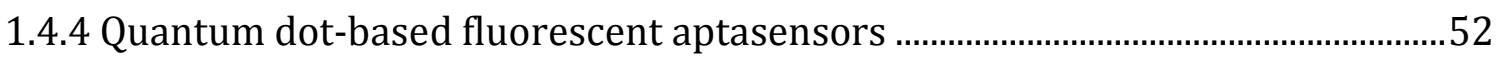

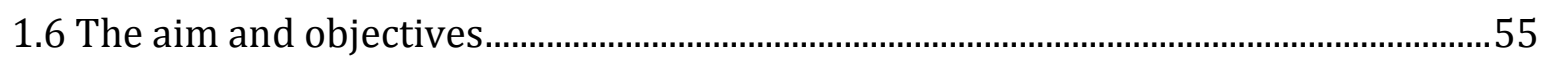

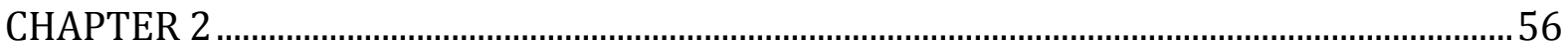

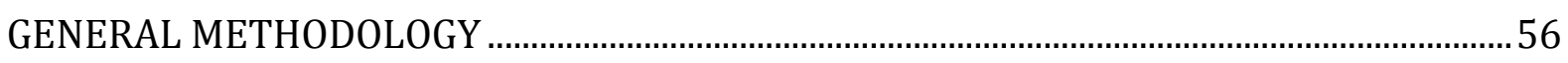

2.1 Synthesis of affinity matrices for aptamer selection ......................................................56

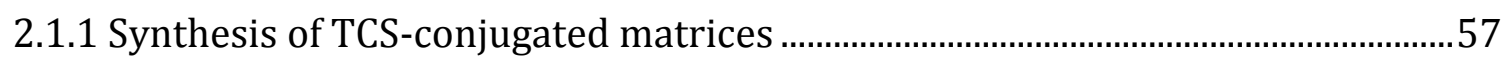


2.1.2 Synthesis of BPA- and OE2-conjugated matrices.....................................................58

2.2 Preparation of ssDNA aptamer library and primers ....................................................59

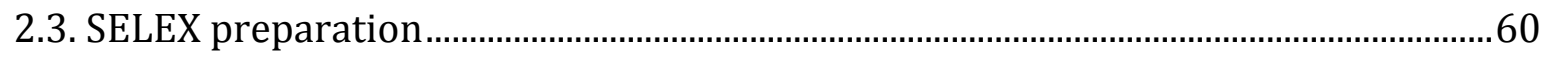

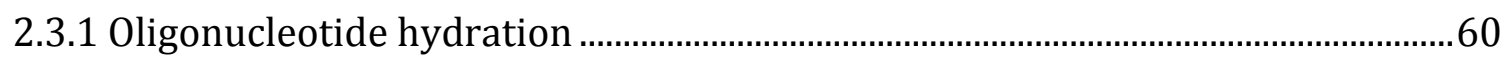

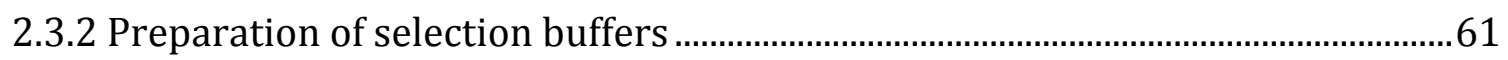

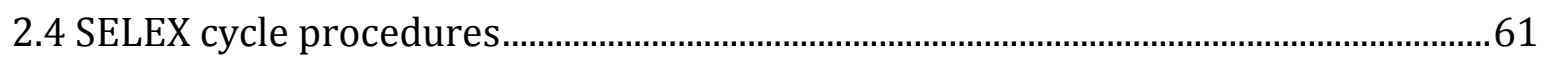

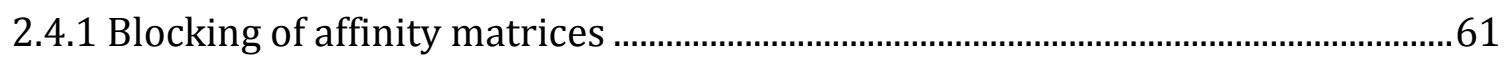

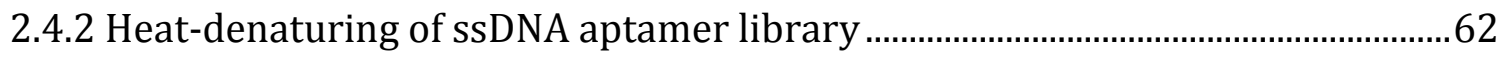

2.4.3 Co-incubation of aptamer library with affinity matrices ..........................................62

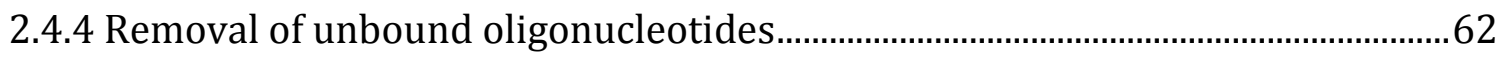

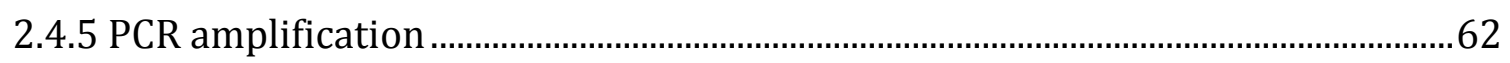

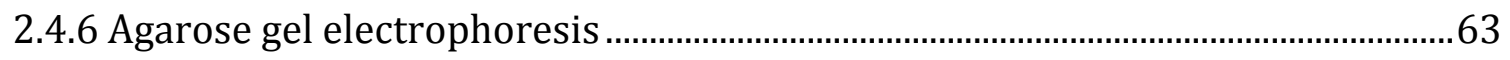

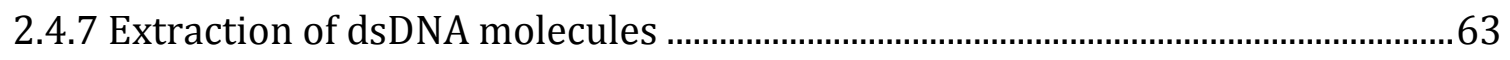

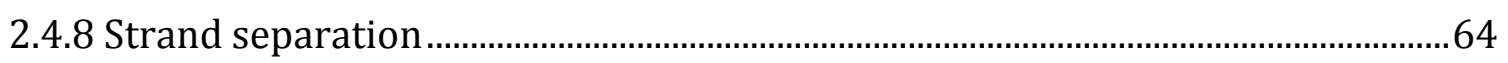

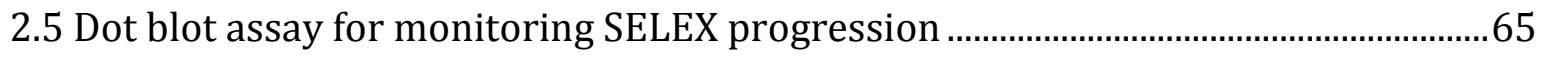

2.5.1 Preparation of nitrocellulose membranes.............................................................65

2.5.2 Incubation with biotin-labelled ssDNA aptamers ....................................................66

2.5.3 Incubation with streptavidin-horse radish peroxidase...........................................66

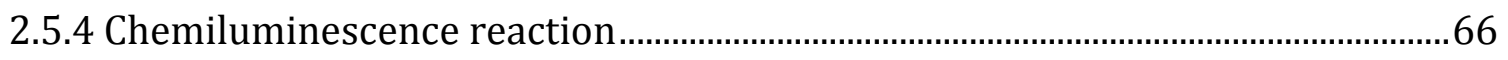

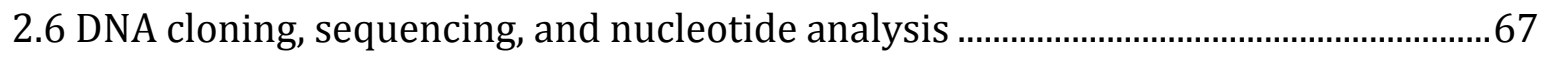

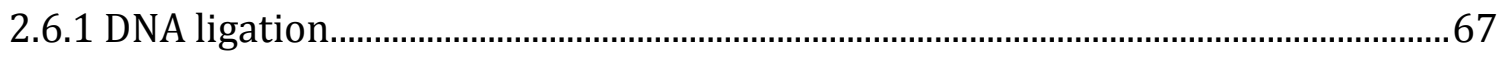

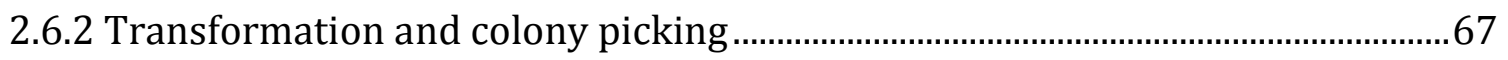

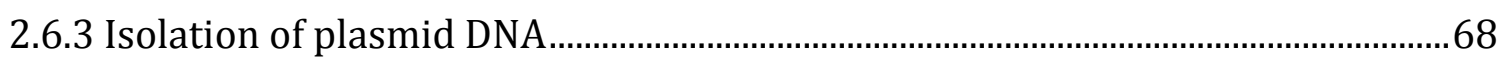

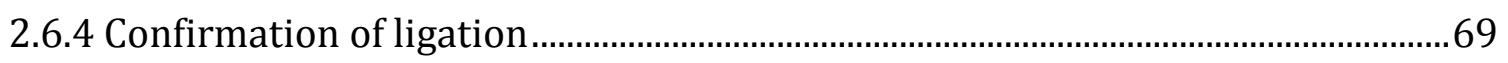

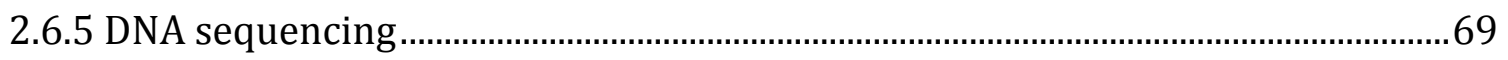

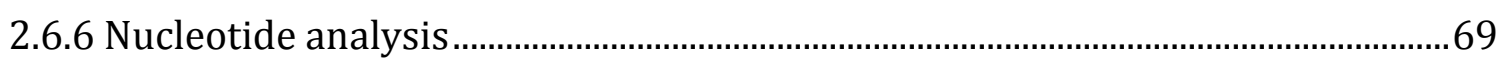

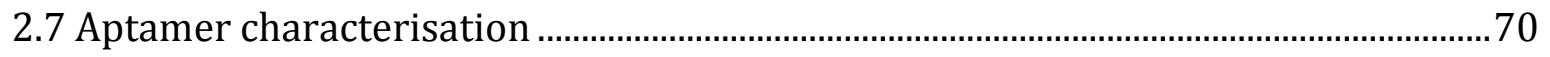

2.7.1 Dot blot assay for binding affinity and specificity ................................................... 70

2.7.2 Fluorescent binding assay for $K_{d}$ determination..................................................... 71

2.7.3 Circular dichroism for assessing conformational change........................................72

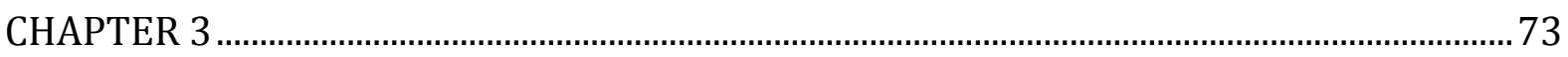

INITIAL SELEX INVESTIGATION AND THE DEVELOPMENT OF A FLUORESCENT

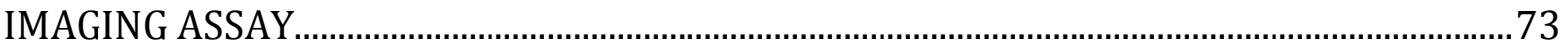

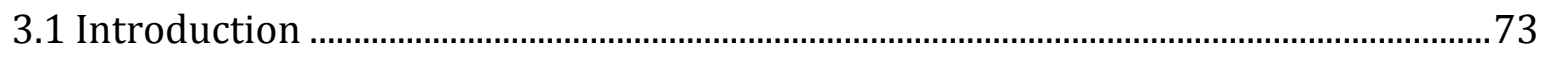

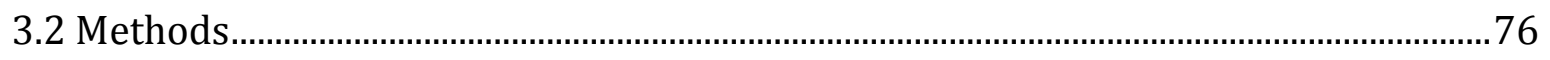

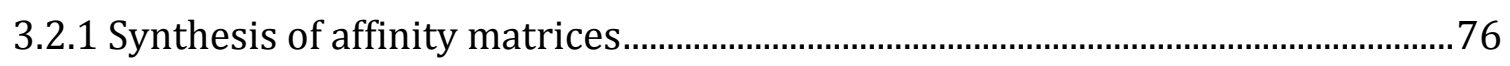

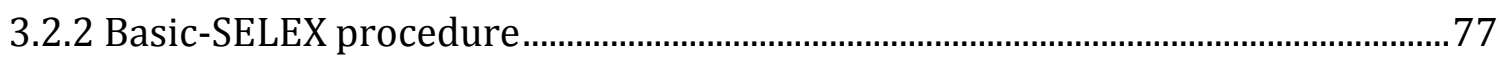

3.2.3 Progression of the basic-SELEX procedure ............................................................. 78

3.2.4 DNA cloning, sequencing and nucleotide analysis ................................................... 79

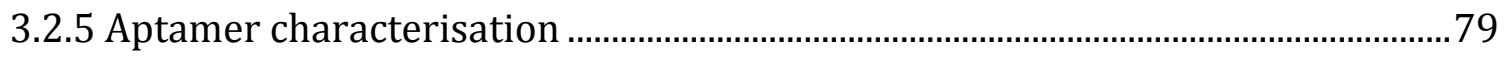


3.2.6 The development of a novel fluorescent imaging assay........................................80

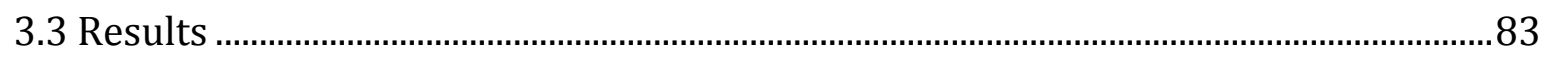

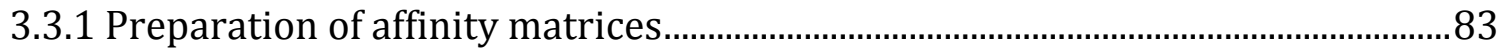

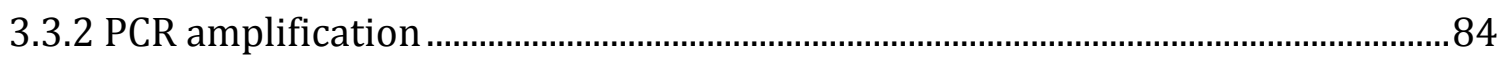

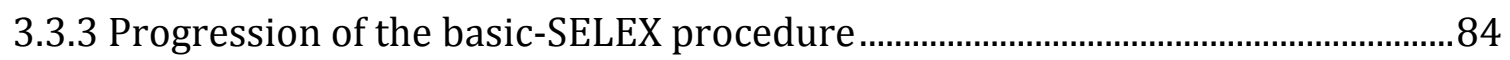

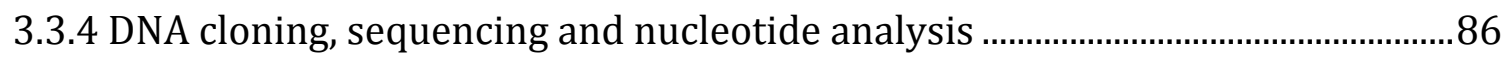

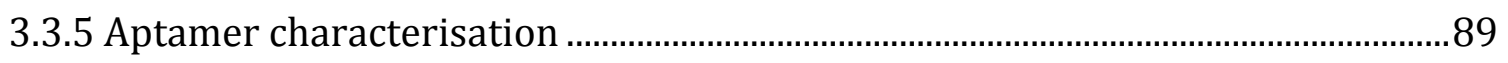

3.3.6 The development of a fluorescent imaging assay ................................................... 96

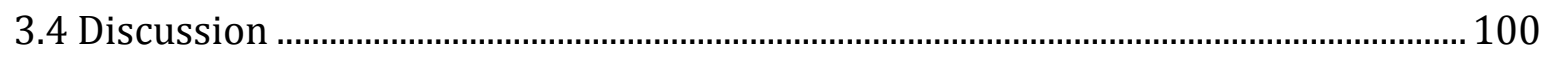

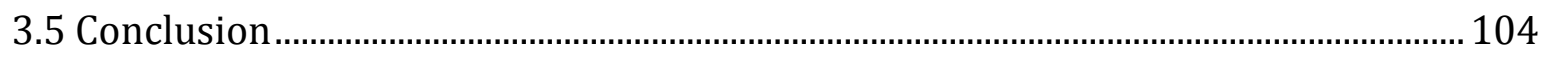

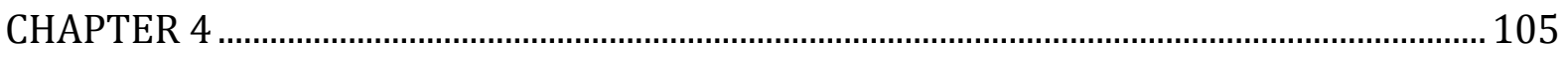

TRICLOSAN APTAMER SELECTION: REVISING THE SELEX METHOD .................................. 105

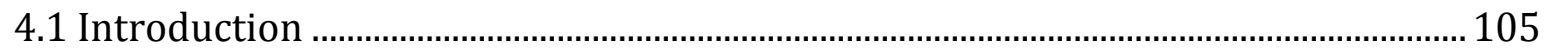

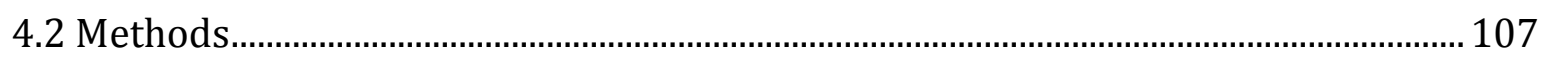

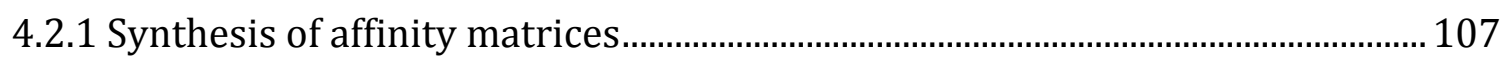

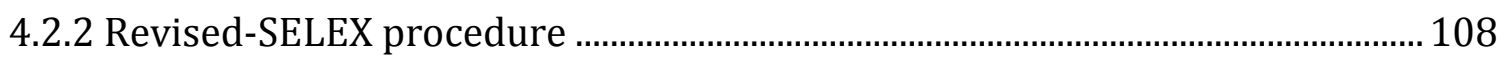

4.2.3 Progression of the revised-SELEX procedure ...................................................... 110

4.2.4 DNA cloning, sequencing and nucleotide analysis ................................................ 111

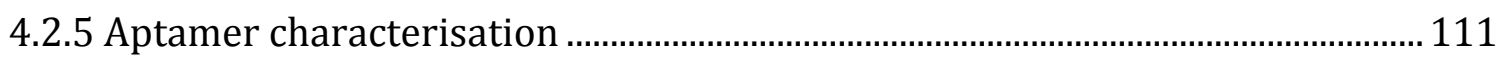

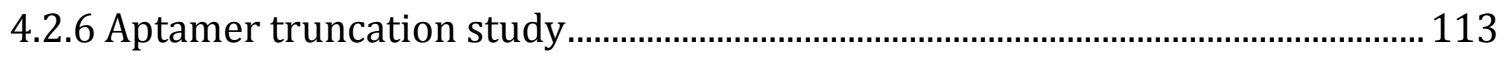

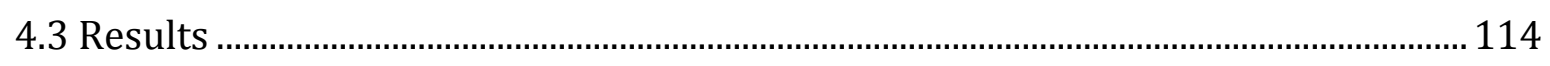

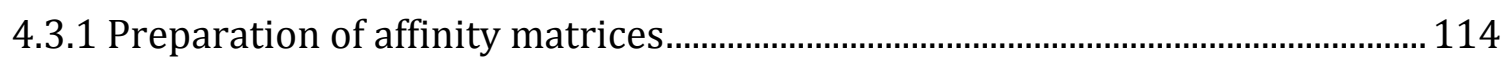

4.3.2 Elucidating the impact of negative and counter selections ................................ 114

4.3.3 Progression of the-revised SELEX procedure ......................................................... 115

4.3.4 DNA cloning, sequencing, and nucleotide analysis ............................................... 117

4.3.5 Aptamer characterisation .............................................................................. 121

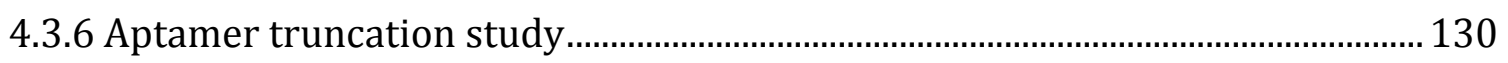

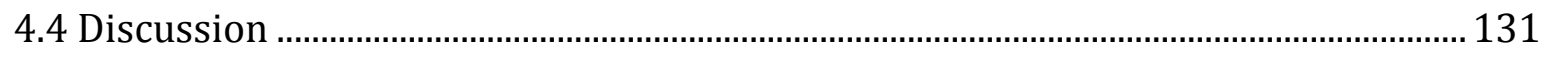

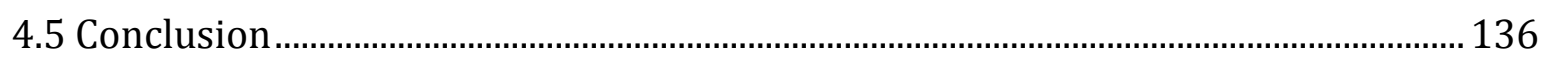

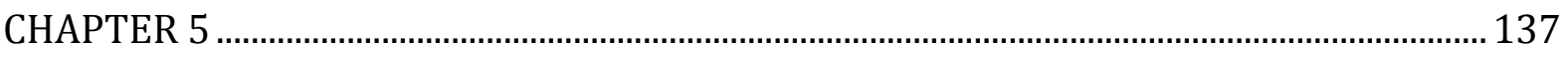

DETECTION OF TRICLOSAN USING A GOLD NANOPARTICLE COLORIMETRIC METHOD

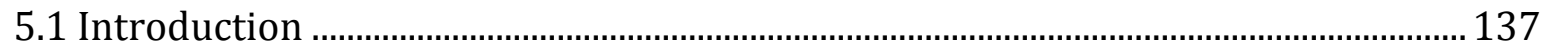

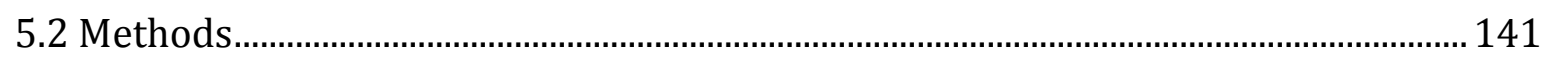

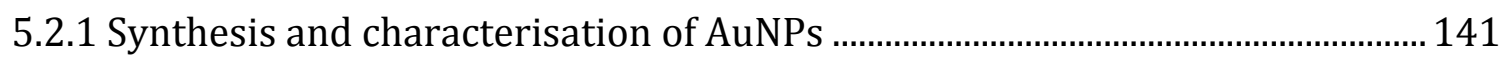

5.2.2 Optimisation of AuNPs for biosensing ……........................................................... 142

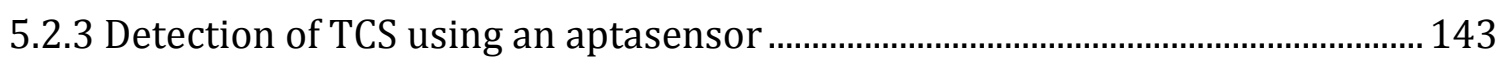

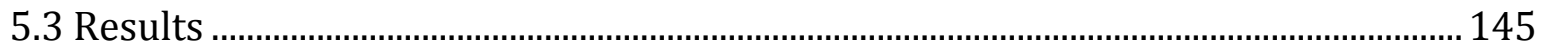

5.3.1 Spectral characteristics of the synthesised AuNPs ............................................. 145 
5.3.2 Optimisation of AuNPs for biosensing .............................................................. 145

5.3.3 Detection of TCS and structurally similar compounds ........................................ 147

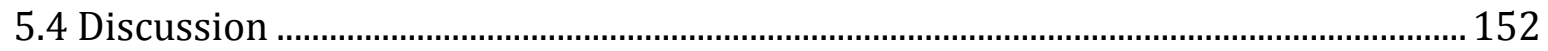

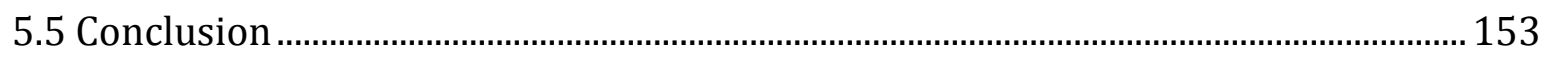

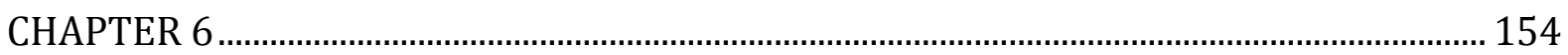

GENERAL DISCUSSION AND FUTURE PERSPECTIVES ....................................................... 154

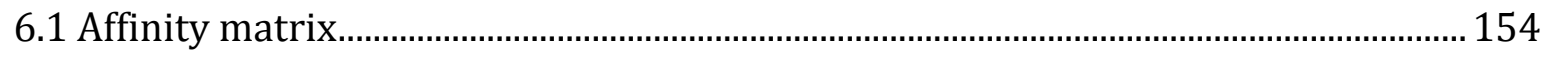

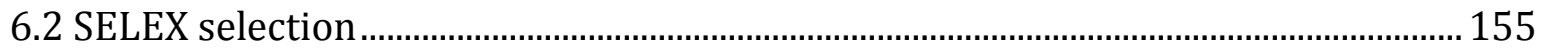

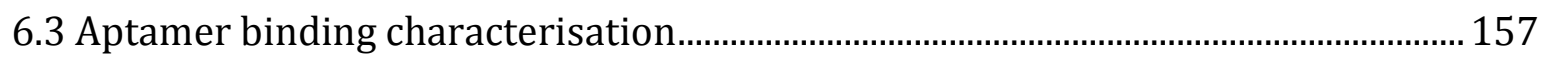

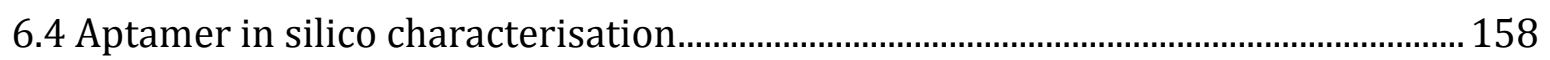

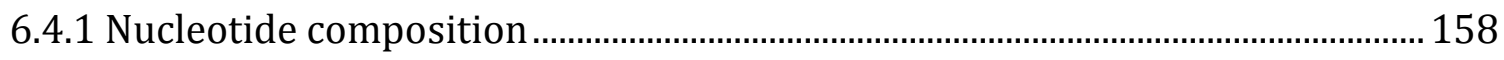

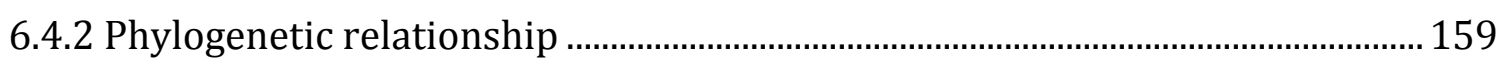

6.4.3 Sequence alignment and secondary structure prediction....................................160

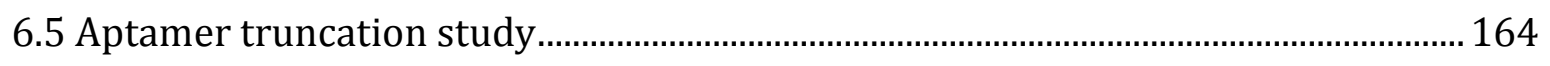

6.6 Aptasensor ..................................................................................................... 164

6.7 Future perspectives................................................................................................. 166

6.7.1 Selection using a doped aptamer library ………………………………………... 166

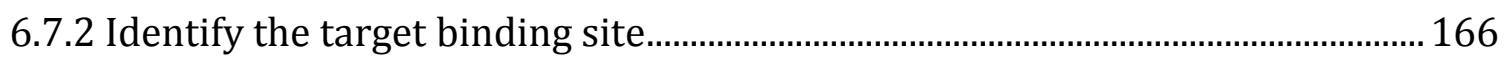

6.7.3 Further use of the developed aptasensor ......................................................... 167

LIST OF REFERENCES ............................................................................................................... 168

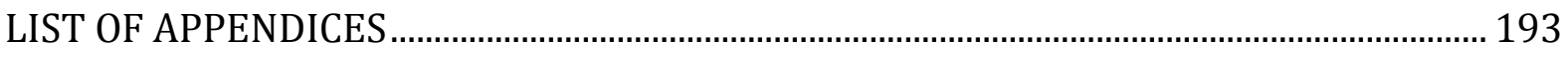

APPENDIX 


\section{LIST OF FIGURES}

Figure

Page

1.1: Molecular structures of selected endocrine disrupting compounds 19

1.2: The common three-dimensional structures assumed by ssDNA aptamers .................26

1.3: Chemical structures of theophylline, caffeine, $L$-arginine and $D$-arginine...................28

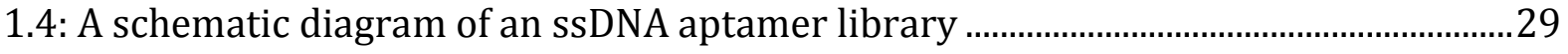

1.5: General scheme of the SELEX procedure .......................................................................... 31

1.6: Aptamer-based sandwich assays: (A) ELONA (enzyme-linked oligonucleotide assay) and (B) ALISA (aptamer-linked immobilised sorbent assay)..................................................49

1.7: General design and mechanism of an acoustic aptasensor ...............................................50

1.8: A structure-switching electrochemical aptasensor .......................................................... 51

1.9: A quantum dot-based aptasensor utilising fluorescent resonance energy transfer

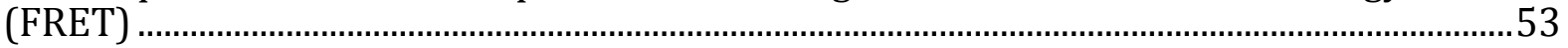

1.10: Chemiluminescence resonance energy transfer (CRET) stimulated within the hemin and G-quadruplex coated quantum dots in the presence of luminol and $\mathrm{H}_{2} \mathrm{O}_{2} \ldots \ldots \ldots \ldots \ldots \ldots . . . .54$

3.1: Compounds with structural similarities to triclosan........................................................ 74

3.2: Conjugation chemistries for the formation of TCS affinity matrices ..............................75

3.3: UV-visible spectra of (A) TCS in water; (B) unmodified sepharose beads (blue), control D matrix (red), and control E matrix (green); (C) TCS D matrix; and (D) TCS E matrix .83

3.4: PCR amplification of the basic-SELEX round 8 aptamer-bound matrices for dot blot assays

3.5: Dot blot assays for polyclonal TCS aptamers from the basic-SELEX rounds 4, 6, and 8 selected using (i) long-arm TCS D matrix; (ii) short-arm TCS E matrix and; (iii) control experiments

3.6: Phylogenetic relationships among TCS round 8 aptamers D (red) and E (blue) clones 87

3.7: Alignment of the $\mathrm{N} 40$ regions for TCS round 8 aptamers (D and E clones) .88

3.8: Secondary structures of aptamers D5 (TR8D5) and E6 (TR8E6) demonstrated a three

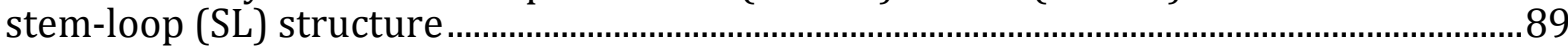

3.9: Binding intensity and sensitivity of TCS round 8 aptamers ( $\mathrm{D}$ and E clones) .............90

3.10: Dot blot assay for screening the binding specificities of TCS round 8 aptamers (i) D4, (ii) D5, (iii) D6, (iv) E5 and (v) E6 to TCS (triclosan), MTS (methyl-triclosan), BPA (bisphenol A), DCF (diclofenac) and OE2 (oestradiol). 
3.11: Saturation curves of (A) aptamer D5 to control and TCS D matrices; (B) aptamer E6 to control and TCS E matrices and; normalised saturation curves of (C) aptamer D5 and (D) aptamer E6 after subtracting the non-specific binding to control matrix from the specific binding to TCS-conjugated matrix

3.12a: Fluorescent microscope operation for capturing fluorescent images from HEXlabelled aptamer E6 (HEX-E6) binding to control and TCS E matrices 96

3.12b: Image analysis of HEX-labelled aptamer E6 (HEX-E6) binding to control and TCS E matrices

3.13a: Fluorescent images showing affinities of TCS round 8 (R8) aptamers D4, D5, D6, E5 and E6 to control and TCS-conjugated matrices 98

3.13b: Fluorescent counts representing binding affinities of TCS round 8 (R8) aptamers to control and TCS-conjugated matrices

3.14a: Fluorescent images of HEX-labelled TCS aptamer D5 (HEX-D5) binding to control and TCS D matrices at 8 concentrations ranging from 40 to $1000 \mathrm{nM}$.

3.14b: Saturation curves of (i) TCS aptamer D5 to control and TCS D matrices, and (ii) a normalised saturation curve of D5 by subtracting the non-specific binding to control matrix from the specific binding to TCS D matrix. . .99

3.15: Fluorescent images of HEX-labelled aptamers D5-bound TCS D matrix and E6bound TCS E matrix after elution. 100

4.1: UV-visible spectra of (A) free BPA in water and (B) BPA-matrix in water 114

4.2: Real-time PCR amplification of aptamer-bound positive, negative and counter selection matrices from the revised-SELEX rounds 3,6 , and 8 . 115

4.3a: Fluorescent images of the aptamer-bound TCS D matrix from each revised-SELEX round.

4.3b: Fluorescent counts representing the binding affinity of selected aptamer library at each revised-SELEX round to TCS D matrix

4.4: Phylogenetic relationships among TCS round 10 aptamers (H1 - H10) ................... 118

4.5: Alignment of $\mathrm{N} 40$ regions for TCS round 10 aptamers (H1 - H10)

4.6: Secondary structures of aptamers H2 (TR10H2), H6 (TR10H6) and H5 (TR10H5) demonstrating two, three and four stem-loop (SL) structures respectively.

4.7a: Fluorescent images showing affinities of the random (Rand) sequence and TCS round 10 (R10) aptamers (H1 - H10) to control and TCS D matrices.

4.7b: Fluorescent counts representing binding affinities of the random (Rand) sequence and TCS round 10 (R10) aptamers to control and TCS D matrices

4.8a: Fluorescent images of HEX-labelled aptamers (H2, H5 and H6) and the random sequence (Rand) prepared at 20-750 nM, binding to control and TCS D matrices........ 124

4.8b: Saturation curves of TCS aptamers H6 (i), H5 (ii), H2 (iii) and the random sequence (Rand) (iv) to TCS D matrix determined using the fluorescent imaging assay ................. 125 
4.8c: Saturation curves of TCS aptamers H6 (i), H5 (ii), H2 (iii) and the random sequence (Rand) (iv) to TCS D matrix determined using the fluorescent binding assay .................. 126

4.9: CD spectra (A to F) of $1 \mu \mathrm{M}$ TCS aptamers (H2, H5, and H6) and the random sequence (Rand), and also the independent detection of TCS at 2, 5 and $10 \mu \mathrm{M}$ 127

4.10a: Fluorescent images showing reduced fluorescent intensity of the H6 aptamerbound matrix at the presence of competitive reagents in solution 128

4.10b: Binding specificity of the H6 aptamer as assessed in competitive binding studies 129

4.11a: Fluorescent images of HEX-labelled aptamers 57H6F, 58H6R and 40H6B prepared at 20-750 $\mathrm{nM}$, binding to control and TCS D matrices 130

4.11b: Saturation curves of the truncated sequences 57H6F, 58H6R and 40H6B binding to TCS D matrix determined using the fluorescent imaging assay 131

5.1a: An aptasensor utilising cross-linked gold nanoparticles.............................................. 138

5.1b: An aptasensor utilising uncross-linked gold nanoparticles.......................................... 139

5.1c: An aptasensor utilising aptamer-protected AuNPs ...................................................... 140

5.2: Spectral characteristics of the synthesised AuNPs (A) UV-visible adsorption spectra and; (B) a transmission electron microscope image.

5.3: Salt tolerance test of the synthesised AuNPs........................................................................ 146

5.4: The effect of increasing concentrations of TCS in the presence of AuNPs ................. 146

5.5: Optimisation of the amount of the $\mathrm{H} 6$ aptamer required for protecting AuNPs against $26.7 \mathrm{mM}$ salt-induced aggregation. 147

5.6a: Qualitative detection of TCS by observing colour changes due to the formation of AuNP aggregates 147

5.6b: Quantitative UV-visible spectrophotometric detection of TCS in solution using the H6 aptamer-protected AuNPs. 148

5.6c: Correlation between the absorption ratio and the concentration of TCS (20-750 nM) 149

5.7a: Qualitative determination of the specificity of TCS aptasensor.. 150

5.7b: Quantitative UV-visible spectrophotometric detection of the specificity of TCS aptasensor 150

5.7c: Non-specific responses of TCS aptasensor to compounds (MTS, BPA and OE2) that are structurally similar to TCS. 151 


\section{LIST OF TABLES}

Table

$\underline{\text { Page }}$

1.1: Determination of endocrine-disrupting compounds in biological and environmental samples 23

2.1: Nucleotide sequences of the N40 library and primers used in the SELEX procedure 60

2.2: Primer combinations used at different stages of the basic-SELEX procedure 60

3.1: Aptamer selection conditions used in the basic-SELEX procedure .79

3.2: Nucleotide sequences of basic-SELEX round 8 TCS aptamers (Clones D and E) and the composition (\%) of individual bases (A, T, G, and $\mathrm{C}$ ) in the $\mathrm{N} 40$ random region within each aptamer. . .86

4.1: Primer combinations used at different stages of the revised-SELEX procedure..... 108

4.2: Aptamer selection conditions used in the revised-SELEX procedure 110

4.3: Nucleotide sequences of the truncated TCS aptamer H6 with their given identities (IDs): 57H6F, 58H6R and 40H6B.

4.4: Nucleotide sequence of revised-SELEX round 10 TCS aptamers (H1 - H10) and the composition (\%) of individual bases (A, T, G and C) in the $\mathrm{N} 40$ random region within each aptamer.

6.1: Summary of methods and conditions used for aptamer selection 156

6.2: Summary of $K_{d}$ values demonstrated by TCS-specific aptamers 157 


\section{LIST OF ABBREVIATIONS}

2D

3D

ALISA

APEOs

AuNP

bp

BPA

BWB

$\mathrm{Ca}^{2+}$

CD

CRET

DCF

DE

$\mathrm{dH} 2 \mathrm{O}$

dITPs

dsDNA

DNA

DNAse

dNTPs

EB

$\mathrm{ECH}$

EDCs

EDTA

ELISA

ELONA

EPA

EtOH

EU

FB1

FC

FDA

FRET

$\mathrm{g}$

GC-MS²

hrs

HPLC

$\mathrm{K}^{+}$

$K_{d}$

$\mathrm{kg}$

$\mathrm{L}$

LB two-dimensional

three-dimensional

aptamer-linked immobilised sorbent assay

alkylphenol ethoxylates

gold nanoparticle

base-pair

bisphenol A

binding and washing buffer

Calcium ions

circular dichroism

chemiluminescence resonance energy transfer

diclofenac

1,4-butanediol diglycidyl ether

distilled water

doxy inositol triphosphate

double-stranded DNA

deoxyribonucleic acid

deoxyribonuclease

deoxynucleoside triphosphates

elution buffer

epichlorohydrin

endocrine-disrupting compounds

ethylenediamine tetraacetic

enzyme-linked immunosorbent assay

enzyme-linked oligonucleotide assay

Environmental Protection Agency

ethanol

Europe Union

fumonisin B1

fluorescent counts

Food and Drug Administration

fluorescence resonance energy transfer

gravity

gas chromatography-tandem mass spectrometry

hours

high performance liquid chromatography

Potassium ions

equilibrium dissociation constant

kilogram

litre

luria broth 


\begin{tabular}{|c|c|}
\hline $\mathrm{LC}-\mathrm{MS}^{2}$ & liquid chromatography-tandem mass spectrometry \\
\hline LOD & limit of detection \\
\hline $\mathrm{Mg}^{2+}$ & Magnesium ions \\
\hline $\mathrm{Mn}^{2+}$ & Manganese ions \\
\hline mg & miligram \\
\hline mins & minutes \\
\hline $\mathrm{mL}$ & mililitre \\
\hline $\mathrm{Na}^{+}$ & Sodium ions \\
\hline NHS-biotin & N-hydroxysuccinimide long chain biotin \\
\hline NMR & nuclear magnetic resonance \\
\hline OD & optical density \\
\hline OE2 & oestradiol \\
\hline osc & oscillations \\
\hline OTA & ochratoxin A \\
\hline PBDEs & polybrominated diphenyl ethers \\
\hline PCB & polychlorinated biphenyl \\
\hline PCPs & personal-care products \\
\hline PCR & polymerase chain reaction \\
\hline QDs & quantum dots \\
\hline ROI & regions of interest \\
\hline $\begin{array}{l}\text { RT } \\
\text { rt-PCR }\end{array}$ & $\begin{array}{l}\text { room temperature } \\
\text { reverse transcription PCR }\end{array}$ \\
\hline SDS & sodium dodecyl sulphate \\
\hline sec & seconds \\
\hline SELEX & systematic evolution of ligands by exponential enrichment \\
\hline SL & stem and loop \\
\hline SMB & streptavidin-coated magnetic beads \\
\hline SPR & surface plasmon resonance \\
\hline ssDNA & single-stranded DNA \\
\hline ST-HRP & strepavidin-horse radish peroxidase \\
\hline TAE & tris-acetate-EDTA \\
\hline TCS & triclosan \\
\hline $\operatorname{Tm}$ & melting temperature \\
\hline TMB & 3,3',5,5'-Tetramethylbenzidine \\
\hline $\mathrm{v} / \mathrm{v}$ & volume per volume \\
\hline WWTPs & wastewater treatment plants \\
\hline $\mathrm{w} / \mathrm{v}$ & weight per volume \\
\hline
\end{tabular}




\section{ABSTRACT}

Triclosan (TCS) is a chlorinated organic compound which, due to its antibacterial properties in vitro, has found widespread usages in many medical and consumer products such as textiles, plastics and personal care products. Humans are directly and chronically exposed to TCS via dermal and mucosal contact from the use of TCS-formulated products such as soap and toothpaste. TCS is classified as an environmental contaminant by the European Union Water Framework Directive, whose mandatory goal is to develop new and simple-to-use analytical methodologies capable of measuring low concentrations of TCS and that are suitable for high-throughput detection.

Synthetically-derived single-stranded oligonucleotides, also known as aptamers, are superior candidates for the development of sensitive and high-throughput biosensing strategies. Biosensors utilising aptamers as molecular recognition elements have showed great promise in a variety of diagnostic and therapeutic applications, especially for the detection of small molecular weight organic compounds such as TCS. The aim of this thesis was to develop aptamers as new capture reagents for TCS, as the first step towards the development of an alternative, user-friendly, diagnostic technique for monitoring TCS in both environmental and biological samples. The objectives of the thesis were to: [i] produce by in vitro selection procedures, TCS binding single-stranded DNA (ssDNA) aptamers; [ii] characterise the selected aptamers and determine their equilibrium dissociation constant $\left(K_{d}\right)$ values and; [iii] evaluate the applicability of the selected aptamers in a biosensing platform.

To achieve these objectives, ssDNA aptamers capable of binding TCS were generated in vitro using a sequential approach known as systematic evolution of ligands by exponential enrichment (SELEX). An affinity column-based SELEX strategy together with a variety of SELEX modifications such as negative and counter selections, real-time amplification and fluorescence quantification were explored for finding TCS specific aptamers. A total of 20 TCS aptamers, ten from 8 rounds of a basic-SELEX procedure, and the other ten from 10 rounds of a revised-SELEX procedure were generated. 
In general, these aptamers showed acceptable levels of sensitivity and specificity to TCS, and the best binding aptamer demonstrated a $K_{d}$ value of $378 \mathrm{nM}$. The $K_{d}$ value is comparable to published $K_{d}$ values for compounds that share similar chemical structures to TCS. In addition, a novel fluorescent-based imaging method was developed in this dissertation. The method developed provides an alternative approach for monitoring SELEX progression and has the potential to simplify the way to characterise the binding properties of an aptamer to its cognate target. The utility of this method was compared with commonly used methods such as dot blot and fluorescent binding assays. The performance of the new imaging method was superior to the existing methods in terms of accuracy, simplicity and reproducibility. Furthermore, the best binding TCS aptamer was evaluated for its utility in an aptamer-based biosensor. The developed aptasensor, utilising a TCS aptamer as the recognition element and gold nanoparticles (AuNPs) as the signal reporter, was capable of detecting TCS in spiked-water samples at concentrations ranging from 20-750 nM with a visual detection limit of $150 \mathrm{nM}$.

In conclusion, methods were developed to select, refine, and characterise ssDNA aptamers capable of binding to TCS, and these aptamers have the potential to offer a sensitive, simple-to-use, and user-friendly analytical method for TCS detection. 


\section{CHAPTER 1 \\ GENERAL INTRODUCTION}

Global production of chemically-synthesised organic compounds has increased dramatically over the past decades. Many of these have been beneficial for mankind, but often they are potentially harmful or have toxic properties. Moreover, many persist for long periods in the environment and as a consequence can accumulate within living organisms (Daughton and Ternes 1999; Daughton 2004). In addition to their persistence, and risk of bioaccumulation, these substances are known to adversely affect biological systems in plants and animals, including humans (Crisp et al. 1998). Therefore it is important to develop suitable methods for detecting and monitoring the presence of these compounds in living organisms and ecosystems in order to support rational decisions regarding their usage. From this perspective, the work in this thesis focuses on the development of a novel aptamer-based biosensor for assessing the levels of organic compounds that exhibit varying degrees of endocrine-disrupting properties. The following describes: [i] a list of selected endocrine-disrupting compounds; [ii] a summary of using aptamers for molecular recognition; [iii] a technique for generating ssDNA aptamers; [iv] a review on aptamer-based biosensors and; [v] the aim and objectives.

\subsection{Endocrine-disrupting compounds (EDCs)}

Compounds that interfere with the endocrine system and disrupt physiological functions of endogenous hormones are called EDCs. EDCs are known to elicit their responses by modifying the synthesis, secretion, transportation and/or binding of endogenous hormones responsible for the regulation of development and maintenance of cellular homeostasis (Crisp et al. 1998). Sources of EDCs include natural and synthetic hormones, pesticides, polychlorinated biphenyls, phthalates, alkylphenol ethoxylates, dioxins, flame retardants, parabens, bisphenol A, and triclosan (Richardson and Ternes 2011). Factors contributing to the presence of EDCs in the environment are leaching or discharges from industrial or agricultural processes; transfer of contaminants from manufactured household goods and; degradation or leaching from landfills. 
Wastewater treatment plants (WWTPs) are major contributors to the EDC pollution, and these chemicals are found in effluents from WWTPs, and thus can access adjacent surfaces and the ground water (Legler et al. 2002; Schwarzenbach et al. 2006). The fact that EDCs are ubiquitous in the environment, especially in aquatic ecosystems, has raised concerns. The occurrence of chemical compounds that influence the sexual development of fish in English rivers was reported over 20 years ago (Purdom et al. 1994). Since then, many studies have demonstrated the negative effects of EDCs on wildlife and human health, which seem to occur even at concentrations as low as $0.1 \mathrm{ng} / \mathrm{L}$ (Kannan et al. 2003; Koh et al. 2005; Campbell et al. 2006). Moreover, some EDCs possess diverse characteristics such as antiandrogenic as well as steroidogenic activities (Leijs et al. 2009; Brausch and Rand 2011). There are great variabilities in molecular structures of EDCs (Figure 1.1), and the ability to monitor all of these reagents for potential adverse effects is a major challenge. In particular, this research has focussed on EDCs more commonly found in environmental and biological samples that differ in their properties and origins namely: [i] steroid hormones; [ii] brominated flame retardants; [iii] alkylphenol ethoxylates; [iv] personal-care products and; [iv] triclosan.

\subsubsection{Steroid hormones}

Natural and synthetically-derived steroid hormones such as oestrone, oestradiol (OE2), progesterone and testosterone, are considered to be the most potent EDCs present in the environment with proven effect on the biological activity of organisms upon exposure (Mills and Chichester 2005). Examples of possible adverse effects of steroid hormones include feminisation in fish, decreased sperm quality and number in human males, and increased prevalence of breast cancer and reproductive abnormalities, such as miscarriages, irregularities of the menstrual cycle, and infertility in human females (Larsson et al. 1999; Amaral Mendes 2002; Waring and Harris 2005). More detailed information concerning the fate and distribution of steroids and their metabolites (e.g. steroid conjugates) in the environment is important because of their accessibility to living organisms via sewage treatment plants or the leaching of wastewater into soils and water-ways (Brion et al. 2004; Gregoraszczuk et al. 2008). Thus, there has been increasing interest in developing more sensitive and simpler methods to detect steroidal compounds in recent years. 
Figure 1.1: Molecular structures of selected endocrine disrupting compounds.

(i) Steroid hormones

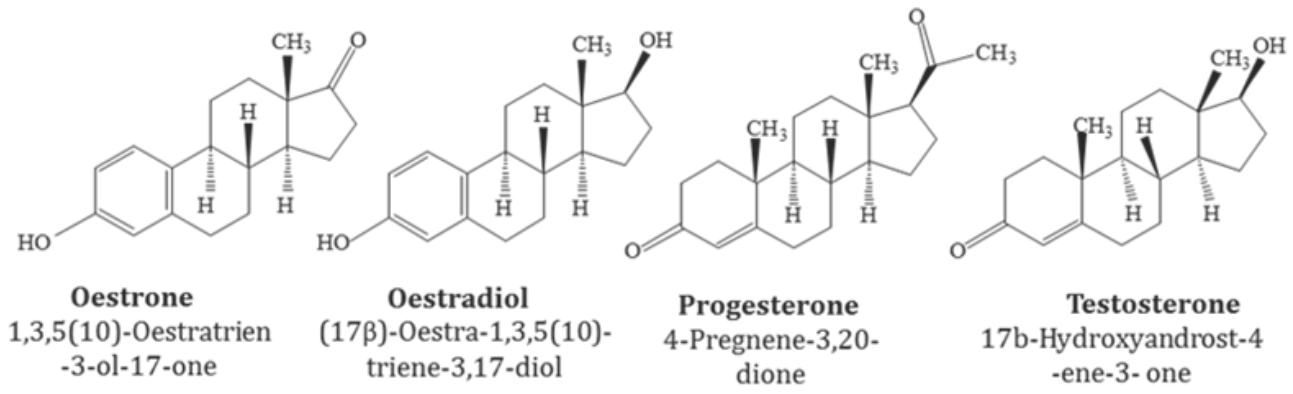

(ii) Brominated flame retardants<smiles>CC(C)(c1cc(Br)c(O)c(Br)c1)c1cc(Br)c(O)c(Br)c1</smiles>

TBBPA

$2,2^{\prime}, 6,6^{\prime}-$

TetrabromobisphenolA<smiles>BrC(Br)CCC(Br)C(Br)CCC(Br)C(Br)CCC(Br)Br</smiles>

HBCD

$1,2,5,6,9,10$

Hexabromocyclododecane<smiles>BrC1=C(Br)C(Br)C2Oc3c(Br)c(Br)c(Br)c(Br)c3[C@H](Br)C2=C(Br)C1Br</smiles>

BDE-100 $2,2^{\prime}, 4,4^{\prime}, 6-$ Pentabromodipheny ether

(iii) Alkylphenol ethoxylates<smiles>CCCCCCCCCc1ccc(O)cc1</smiles>

Nonylphenol

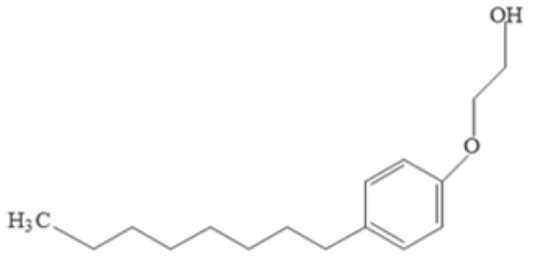

4-Octylphenol

(iv) Personal care products<smiles>O=C(Nc1ccc(Cl)cc1)Nc1ccc(Cl)c(Cl)c1</smiles>

Triclocarban

$\mathrm{N}$-(4-Chloropheny)-N-(3,4dichlorophenyl) urea<smiles>COC(=O)c1ccc(O)cc1</smiles>

Methylparaben<smiles>COc1ccc(C(=O)c2ccccc2)c(O)c1</smiles>

Benzophenone-3

2-Hydroxy-4-methoxy

benzophenone

(iv) Triclosan and its derivatives<smiles>Oc1cc(Cl)ccc1Oc1ccc(Cl)cc1Cl</smiles>

Triclosan

5-Chloro-2-(2,4dichlorophenoxy)phenol<smiles>COc1cc(Cl)ccc1Oc1ccc(Cl)cc1Cl</smiles>

Methyl tridosan

2,4,4'-Trichloro-2'methoxydiphenyl ether<smiles>Oc1ccc(Cl)cc1O</smiles>

2,4-Dichlorophenol 4-Chlorocatechol

A common characteristic to most EDCs is the presence of at least one aromatic moiety in their molecular structures. 


\subsubsection{Brominated flame retardants}

Brominated flame retardants including polybrominated diphenyl ethers (PBDEs), polybrominated biphenyls, brominated cyclohydrocarbons, decabromodiphenyl ethers, hexabromocyclododecanes, and tetrabromobisphenol A, have been used for many years in a variety of commercial products, including children's sleepwear, foam cushions in chairs, computers, plastics, and electronics (Covaci et al. 2011). The use of these flame retardants has led to their widespread presence in the environment. They are environmentally persistent and lipophilic, and therefore can bioaccumulate in both animals and humans (Vonderheide 2009). Because of their widespread presence and potential toxicity to mammals, the Euoropean Union (EU) has banned the use of pentaand octa-PBDEs in 2004. However, deca-PBDEs are still being manufactured and used. Research now shows that the deca-PBDEs can accumulate in animal and human tissues, and metabolically form the toxic penta- and octa-species (Richardson and Ternes 2011).

\subsubsection{Alkylphenol ethoxylates}

Alkylphenol ethoxylates (APEOs) are a class of non-ionic surfactants that are used extensively as detergents, emulsifiers, wetting and dispersing agents in industrial, agricultural, and household applications. Because alkylsubstituted phenols are relatively polar due to the presence of hydroxyl groups, they are highly soluble in aqueous solutions, thereby increasing their potential to pollute water sources (Ferguson and Brownawell 2003). APEOs are known to degrade in WWTPs to metabolites nonylphenols or 4octylphenols. These metabolites are more toxic, lipophilic and oestrogenic in nature, and persist in the environment than the parent compounds (Vega Morales et al. 2009). In the past 20 years, several studies have reported that this class of compounds bioaccumulate in aquatic organisms resulting in chronic toxicity (Staples et al. 2004). Moreover, these compounds are known to mimic natural hormones and disrupt endocrine functions by interacting with oestrogen receptors (Jobling et al. 2006; Lu et al. 2008). 


\subsubsection{Personal-care products}

Personal-care products (PCPs) are the focus of much research because of their potential impacts on the environment and human health, as well as the increased public awareness and concern. Despite the growing availability of PCPs, few studies have demonstrated their potential biological and ecological impacts to living organisms (Subedi et al. 2012; Ortiz de Garcia et al. 2013). Even fewer studies have addressed the potential human health relevance of these and other non-regulated xenobiotics (Kumar and Xagoraraki 2010). Through the use of PCPs, humans are continually and directly exposed to synthetic musks (e.g. nitro-musk, galaxolide, and tonalide) used in the perfume industry, preservatives (e.g. triclocarban and methyl-paraben) for personal hygiene, sunscreen filters (e.g. oxybenzone), and insect repellents (e.g. metofluthrin, and icaridin). PCPs are lipophilic and due to constant exposure persist in human tissues. Some studies have suggested that the half-life of these compounds are in the order of several months (Hutter et al. 2010).

\subsubsection{Triclosan (TCS)}

TCS is a broad spectrum antimicrobial and preservative agent that is used in a wide range of products including antibacterial soaps, toothpaste, shampoo, lotion, and deodorants. TCS can also be incorporated into polymers and fibres to provide materials with antimicrobial properties. It is frequently used in mattress pads, food cutting boards, household sponges, rubbish bags and plastic rubbish bins (Saleh et al. 2010). The annual usage of TCS is increasing with the rising consumer demand for hygienic products (Singer et al. 2002). It has been estimated that the annual usage of TCS is $>300$ tons in USA and 350 tons in Europe (Singer et al. 2002; Halden and Paull 2005). Extensive use of TCS increases the potential for general population to be exposed to TCS through ingestion or dermal contact or through consumption of contaminated food and drinking water. 
TCS has been identified in human breast milk at levels between 100 to 2,100 $\mu \mathrm{g} / \mathrm{kg}$ lipids (Dayan 2007), plasma samples at levels just under $10 \mathrm{ng} / \mathrm{mL}$ (Sandborgh-Englund et al. 2006), and in samples of urine at levels approaching $4000 \mathrm{ng} / \mathrm{mL}$ (Calafat et al. 2008). These data indicate quite clearly that humans are being exposed to TCS at levels that enable the molecule to bioaccumulate and become readily available in various excretory fluids. A cause for concern in this regard, is that both in vitro and in vivo studies show that TCS is capable of exerting adverse effects on hormonal homeostasis and metabolic mechanisms (Wong et al. 2001; Gafni et al. 2004; Chen et al. 2007; Ahn et al. 2008; Kumar et al. 2008; Kumar et al. 2009). TCS is structurally similar to polychlorinated biphenyls (PCBs) and has similarly been shown to potentially interact with ryanodine receptors and stimulate cellular $\mathrm{Ca}^{2+}$ mobilisation, thereby adversely affecting neural-development (Ahn et al. 2008; Wong et al. 2001; Gafni et al. 2004). In vitro studies of TCS shows dosedependent antagonism at low micromolar $(\mu \mathrm{M})$ levels towards oestradiol- and testosterone-mediated activation of oestrogen and androgen receptors respectively (Chen et al. 2007). Furthermore, recent studies have shown that the oral administration of TCS at doses of 10 and $20 \mathrm{mg} / \mathrm{kg} /$ day inhibits testicular androgen production in male rats (Kumar et al. 2008; Kumar et al. 2009).

\subsubsection{Current methods of EDC detection}

The detection of EDCs is an essential initial step to evaluate whether their environmental impacts are of concern. However, analyses of EDCs represent a difficult task due to the complexities of the matrices (soils, water, sediments, biological fluids or tissues) being analysed and the available concentrations are often very low (ng/L). The challenge in detecting trace-levels of EDCs in complex sample mixtures requires new analytical methods not only with high sensitivity, but also with different levels of selectivity and resolution (Aufartova et al. 2011). Chromatographic methods such as high performance liquid chromatography (HPLC), liquid chromatography-tandem mass spectrometry (LC$\mathrm{MS}^{2}$ ), and gas chromatography-tandem mass spectrometry (GC-MS${ }^{2}$ ) are the traditionally used techniques for quantitative and qualitative measurement of EDCs (Table 1.1). 
Table 1.1: Determination of endocrine-disrupting compounds in biological and environmental samples

\begin{tabular}{|c|c|c|c|c|c|}
\hline Analytes & Samples & Extraction & Methods & $\begin{array}{l}\text { Analytical } \\
\text { parameters }\end{array}$ & References \\
\hline $\begin{array}{l}26 \text { steroids including natural and synthetic estrogens, progestogens, } \\
\text { and androgens }\end{array}$ & Water samples & SPE & LC-MS² & $\begin{array}{l}\text { Recovery }>80 \% \\
\text { MDLs: } 0.1-0.73 \mathrm{ng} \cdot / \mathrm{L}\end{array}$ & (Vulliet et al. 2008) \\
\hline $\begin{array}{l}\text { Boldenone, nandrolone, testosterone, methyltestosterone, } \\
\text { epiandrosterone, androsterone, and satnozolol }\end{array}$ & Human urine & $\begin{array}{l}\text { In-tube } \\
\text { SPME }\end{array}$ & LC-MS $^{2}$ & $\begin{array}{l}\text { Recovery: } 86-117 \% \\
\text { LODs: } 9-182 \mathrm{pg} / \mathrm{mL}\end{array}$ & (Saito et al. 2010) \\
\hline $\begin{array}{l}\text { Estrone, } 17 \alpha \text {-estradiol, } 17 \beta \text {-estradiol, estriol, } 17 \alpha \text {-ethinylestradiol, } \\
\text { diethylstilbestrol, estradiol } 17 \text {-glucoronide, estrone }\end{array}$ & Sewage sludge & PLE & $\mathrm{LC}-\mathrm{MS}^{2}$ & $\begin{array}{l}\text { Recovery }>81 \% \\
\text { LODs }<26 \mathrm{ng} / \mathrm{g}\end{array}$ & (Nieto et al. 2008) \\
\hline Eighteen androgens and progestogens & $\begin{array}{l}\text { Environmental } \\
\text { waters }\end{array}$ & DLLME & HPLC-MS $^{2}$ & $\begin{array}{l}\text { Recovery: } 87-116 \% \\
\text { LODs: } 0.8-3.1 \mu \mathrm{g} / \mathrm{L}\end{array}$ & $\begin{array}{l}\text { (Chang and Huang } \\
\text { 2010) }\end{array}$ \\
\hline Octyl, nonylphenol ethoxylates, and carboxylates & Wastewater & SPE, LLE & LC-MS $^{2}$ & $\begin{array}{l}\text { Recovery: } 21-71 \% \\
\text { LODs: } 2-29 \mathrm{ng} / \mathrm{L}\end{array}$ & $\begin{array}{l}\text { (Loyo-Rosales et al. } \\
\text { 2007) }\end{array}$ \\
\hline Octyl, nonylphenol ethoxylates and bisphenol A & Bottled water & - & HPLC-MS ${ }^{2}$ & $\begin{array}{l}\text { Recovery: } 97-106 \% \\
\text { LODs: } 0.04-0.057 \mu \mathrm{g} / \mathrm{L}\end{array}$ & $\begin{array}{l}\text { (Wang and Schnute } \\
\text { 2010) }\end{array}$ \\
\hline Bisphenol A & Human milk & SPE & ${\mathrm{GC}-\mathrm{MS}^{2}}^{2}$ & $\mathrm{LOQ}=1.16 \mathrm{mg} / \mathrm{kg}$ & $\begin{array}{l}\text { (Deceuninck et al. } \\
\text { 2015) }\end{array}$ \\
\hline Bisphenol A & Bovine milk & SPE & LC-MS & $\begin{array}{l}\text { Recovery: } 71-97 \% \\
\text { LOD: } 0.20 \mu \mathrm{g} / \mathrm{L}\end{array}$ & (Yan et al. 2009) \\
\hline TBBPA and brominated bisphenol A analogues & $\begin{array}{l}\text { Sediment and } \\
\text { sludge }\end{array}$ & Soxhlet & LC-MS2 & $\begin{array}{l}\text { Recovery: } 70-105 \% \\
\text { LOQs: } 0.02-0.15 \mathrm{ng} / \mathrm{g}\end{array}$ & (Chu et al. 2005) \\
\hline PBDE28, 47, 99, 100, 153, 154 and 183 & Soil & PLE & GC-MS & $\begin{array}{l}\text { Recovery: } 88-110 \% \\
\text { LOD: } 1154 \mathrm{pg} / \mathrm{g}\end{array}$ & (Zhang et al. 2010) \\
\hline 36 halogenated flame retardants & Fish & PLE & LC-MS² & IDLs: $4.7 \mathrm{pg}$ & (Zhou et al. 2010) \\
\hline HBCDs and TBBPA & Sewage sludge & PLE & HPLC-MS ${ }^{2}$ & $\begin{array}{l}\text { Recovery: } 65-112 \% \\
\text { LOQs: } 0.005-0.14 \mathrm{ng} / \mathrm{g}\end{array}$ & $\begin{array}{l}\text { (Mascolo et al. } \\
\text { 2010) }\end{array}$ \\
\hline Triclosan and triclocarban & $\begin{array}{l}\text { Waste and tap } \\
\text { waters }\end{array}$ & DLLME & $\mathrm{LC}-\mathrm{MS}^{2}$ & $\begin{array}{l}\text { Recovery: } 70-103 \% \\
\text { LODs: } 0.04-0.58 \mu \mathrm{g} / \mathrm{L}\end{array}$ & (Zhao et al. 2010) \\
\hline $\begin{array}{l}\text { Triclocarban, triclosan, Methylparaben, ethylparaben, benzylparaben, } \\
\text { and propylparaben }\end{array}$ & $\begin{array}{l}\text { Surface and waste } \\
\text { waters }\end{array}$ & SPE & HPLC-MS ${ }^{2}$ & $\begin{array}{l}\text { Recovery: } 20-101 \% \\
\text { LODs: } 20-200 \mathrm{ng} / \mathrm{L}\end{array}$ & $\begin{array}{l}\text { (Pedrouzo et al. } \\
\text { 2009) }\end{array}$ \\
\hline $\begin{array}{l}\text { Triclosan and triclocarban, methyl paraben, ethyl paraben, propyl } \\
\text { paraben, and benzyl paraben }\end{array}$ & Sewage sludge & PLE & HPLC-MS $^{2}$ & $\begin{array}{l}\text { Recovery: } 15-100 \% \\
\text { LODs < } 8 \mathrm{ng} / \mathrm{g}\end{array}$ & (Nieto et al. 2009) \\
\hline $\begin{array}{l}\text { Benzophenone-1, -2,-3 and -4, 4,4-dihydroxybenzophenone, ethyl-4- } \\
\text { aminobenzoate, 2-ethylhexyl-4-trimethoxycinnamate,3-(4- } \\
\text { methylbenzylidene)-camphor, and 3-benzylidene-camphor }\end{array}$ & Fish & $\begin{array}{l}\text { Ethyl acetate, } \\
\text { n-heptane, } \\
\text { and water }\end{array}$ & LC-MS & $\begin{array}{l}\text { Recovery }>72 \% \\
\text { LODs: } 78-205 \mathrm{ng} / \mathrm{g} \\
1.8-5.3 \mu \mathrm{g} / \mathrm{kg}\end{array}$ & (Zenker et al. 2008) \\
\hline
\end{tabular}

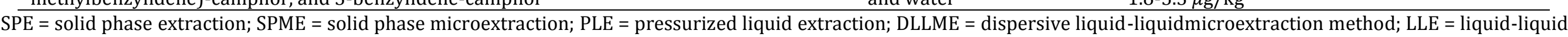

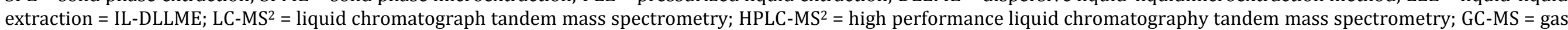

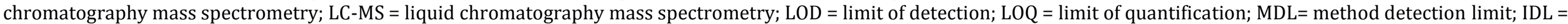
instrument detection limit; TBBPA = tetrabromobisphenol A; PBDE = polybrominated dipheny lethers and; HBCD = hexabromocyclododecanes. 
Although these technologically-sophisticated instruments are sensitive and selective, their analytical processes typically require an extraction step prior to the measurement procedure. This extraction step plays a key role in determining the overall level of analytical performance, and often constitutes the most time- and labour-intensive parts of the analytical process (Shelver et al. 2007). In addition, these methods typically require sophisticated laboratory-based environment and highly trained personnel, and are not really amendable for decentralised analyses. Alternatively, conventional receptor-based assays, such as immunoassays have been developed with some success for the analysis of some EDCs (Ebrahimi 2007; King and Hassell 2008; Cha et al. 2012). These assays utilise antibodies as molecular recognition elements, and are more cost-effective compared with chromatographic techniques, but lag in terms of stability, production time and often specificity. Burn et al. developed an enzyme-linked immunosorbent assay (ELISA) for TCS detection in water samples (Brun et al. 2008). This method not only eliminated the extraction step, but also the use of expensive equipment, and thus significantly reduced the assay costs. However, the polyclonal antibody for TCS raised in the assay is less sensitive compared with the chromatographic methods, and false positive results are inevitable due to the antibody cross-reacting with compounds that have a similar structure to TCS (Brun et al. 2008). Furthermore, antibodies are obtained by immunising animals and therefore, raise ethical concerns which are becoming more prevalent.

The limitations of these existing techniques have led to considerable research efforts towards the generation of alternative molecular recognition elements. One such alternative approach has been to evaluate the potential use of aptamers for molecular recognition due to their ease of production and modification, and be readily integrated into many biosensing platforms (Levy et al. 2005; Choi et al. 2006; Liu and Lu 2006; Freeman et al. 2013). 


\subsection{Molecular recognition using aptamers}

Molecular recognition between two biomolecules plays an essential role in various biological and biomedical applications (Dominy 2008). These molecular recognition or binding events may occur between proteins and small molecules (i.e. small molecular weight compounds) to induce signalling processes (Schoeneberg et al. 2007), between proteins and nucleic acids, such as in DNA/histone interactions (Henikoff and Ahmad 2005), and between nucleic acids and small molecules, such as those displayed by riboswitches (Serganov et al. 2006). Principles mediating these recognitions include the shape of the two interactive binding surfaces, the extent of hydrogen bonding, ionic and dipolar properties, as well as potential solvent interactions, and van der Waals forces (Dominy 2008). Researchers have taken advantage of molecular recognition principles to form the foundation of current target detection methods and modern diagnosis and/or treatment of disease. While antibodies have been the gold standard for molecular recognition elements for several decades, the relatively new technology of aptamers is emerging as a valuable alternative.

\subsubsection{Aptamers}

Aptamers are single-stranded deoxyribonucleic acids (ssDNA) or ribonucleic acids (RNA) that can bind target molecules with high affinity and specificity by folding into unique three-dimensional (3D) structures (Ellington and Szostak 1990). The ability of aptamers to fold into distinct 3D conformations allows for high affinity binding and selectivity for their target. Similar to the molecular recognition that mediates antibody-antigen binding, aptamer-target binding is through intermolecular interactions such as van der Waals forces, hydrogen bonding, electrostatic interactions between charged groups, and Pi-Pi stacking of "flat-structured" aromatic moieties (Hermann and Patel 2000). The 3D structures of ssDNA aptamers (Figure 1.2) possess hairpins (i.e. a combination of stems, loops or bulges), pseudoknots, or G-quadruplexes (Feigon et al. 1996; Piganeau and Schroeder 2003). 
Figure 1.2: The common three-dimensional structures assumed by ssDNA aptamers

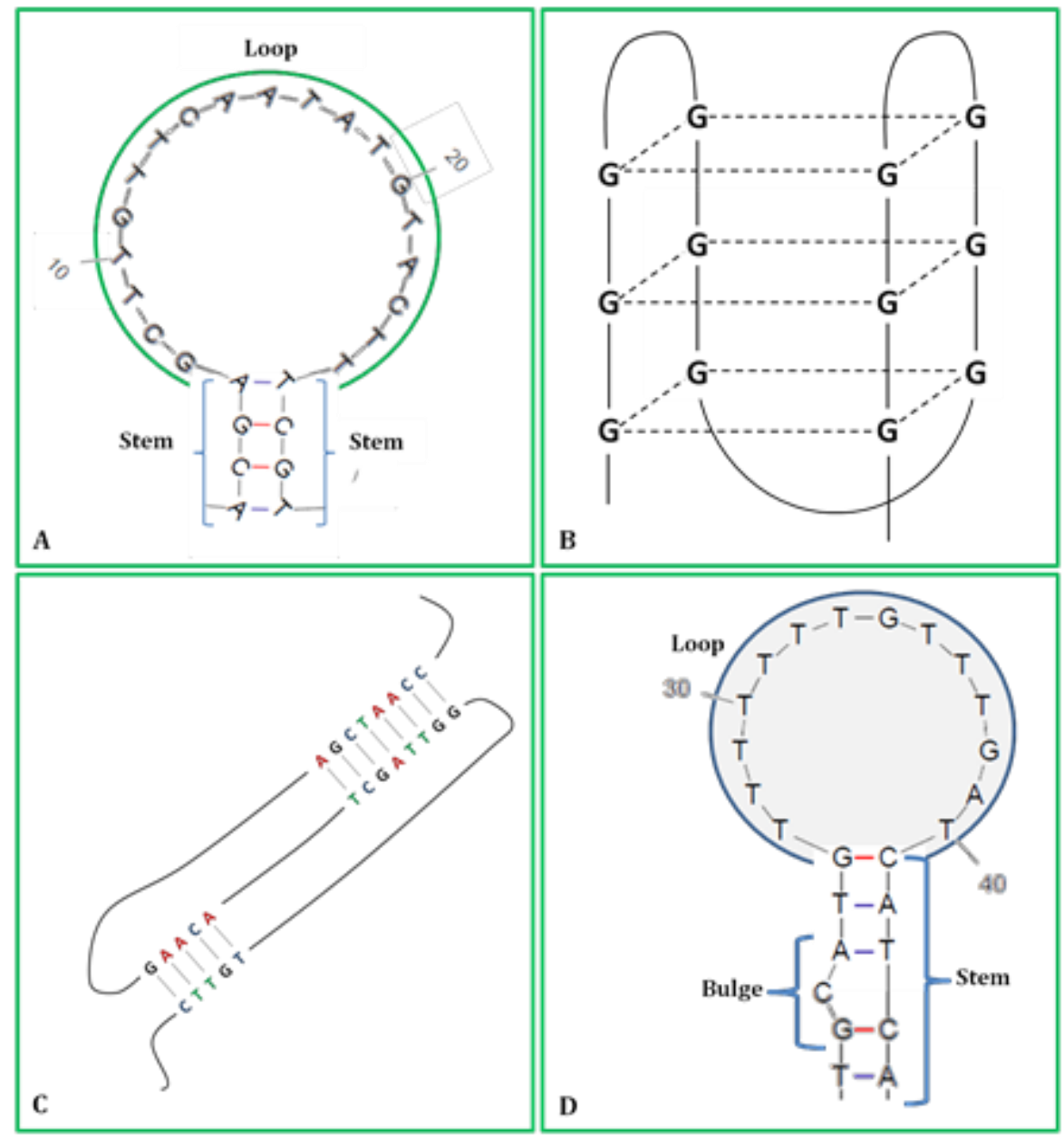

A represents the hairpin structure composed of stem and loop region; B represents the G-quadruplex structure; C represents the pseudoknot structure and; D represents the hairpin structure with a bulge on the stem region. The Figure is reprinted with permission from Dr Shalen Kumar (Victoria University of Wellington, NZ).

Aptamers were first reported 25 years ago by Ellington \& Szostak and Tuerk \& Gold, who independently developed techniques to select RNA molecules against specific organic dyes and T4 DNA polymerase respectively (Ellington and Szostak 1990; Tuerk and Gold 1990). Ellington \& Szostak named the RNA molecule as "aptamer" referring to the Latin word aptus - meaning "to fit", and the Greek word meros - meaning "region" (Ellington and Szostak 1990). Tuerk \& Gold named the technique as systematic evolution of ligands by exponential enrichment (SELEX) (Tuerk and Gold 1990). Both descriptions have become standard terms for aptamer technology. 


\subsubsection{Aptamers versus antibodies}

Aptamers are excellent alternatives or substitutes to antibodies, as they have several important features which are suitable for bioanalytical applications. Aptamers have binding affinities and specificities that are comparable to, and in some cases, even surpass those of monoclonal antibodies. For example, aptamers have been generated with high target structural specificity and enantioselectivity which make them possible to discriminate a specific molecule from its analogues (Jenison et al. 1994; Geiger et al. 1996). The theophylline aptamer developed by Jenison et al. showed an affinity for its cognate ligand that is 10,000 -fold higher than that for caffeine, which differs from theophylline by only a single methyl group at nitrogen atom N-7 (Figure 1.3) (Jenison et al. 1994). The arginine aptamer developed by Geiger et al. showed a 12, 000 fold stronger affinity with $L$-arginine than with $D$-arginine (Figure 1.3) (Geiger et al. 1996). Aptamers are not immunogenic or toxic in vivo, because nucleic acids are not typically recognised by the human immune system as foreign agents (Ireson and Kelland 2006). Thus aptamers are very useful as drug candidates. Aptamers are generally smaller in size compared with antibodies, have better tissue penetration and are more easily internalised by target cells, thereby improving their therapeutic value (Xiang et al. 2015). Aptamers can be developed in vitro against a seemingly unlimited range of targets. To date, specific aptamers against highly diverse-range targets have been developed, such as ions (Ciesiolka et al. 1995), small molecules (Li et al. 1996), neurotransmitters (Mannironi et al. 1997), organic pollutants (Joeng et al. 2009; Jo et al. 2011), drugs (Lato et al. 1995; Wallis et al. 1997), peptides (Welch et al. 1997; Fukusaki et al. 2001) , proteins (Paborsky et al. 1993; Mendonsa and Bowser 2004), and even complex cells or tissues (Daniels et al. 2003; Mallikaratchy et al. 2007). Aptamers are stable under a wide range of temperature and buffer conditions and are resistant to harsh treatments such as physical or chemical denaturation without any significant loss of activity. These features are important for long-term applications of aptamers and also require less restrictive transporting conditions compared with antibodies. 
Figure 1.3: Chemical structures of theophylline, caffeine, $L$-arginine and $D$-arginine

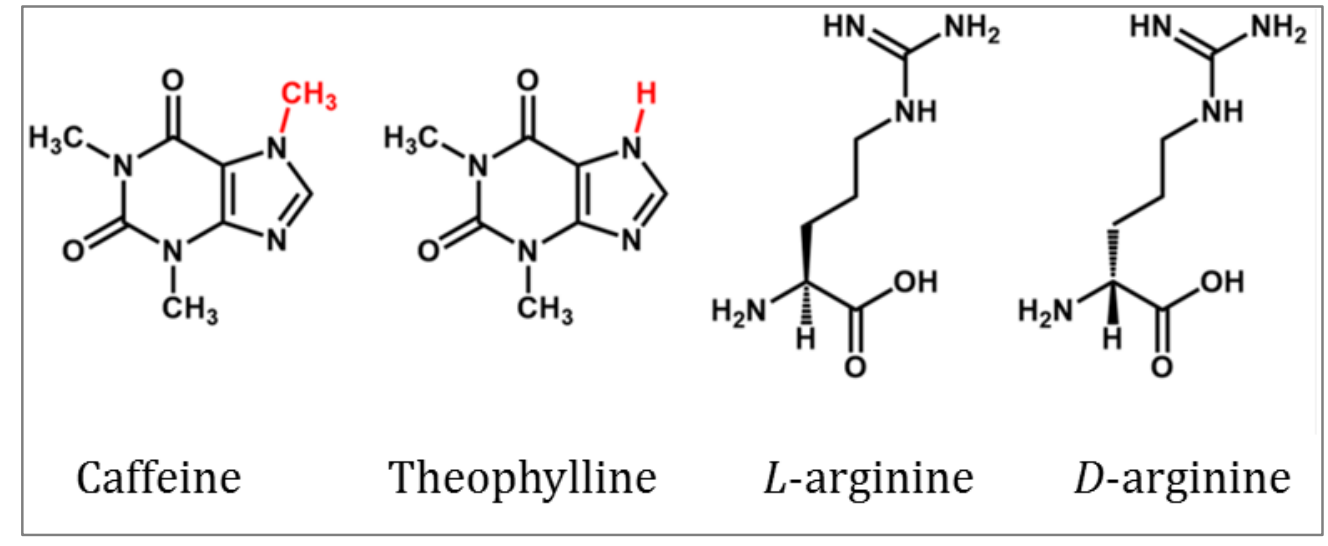

Finally, aptamers can be chemically synthesised using well-established synthetic chemical technologies and a given aptamer can be produced or modified in large scale, with minimal batch-to-batch variation.

\subsubsection{Applications of aptamers}

Applications of aptamers are so numerous that studies describing their use appear in the literature on a weekly basis. The versatility of aptamer technology is reflected in the fact that there are few areas of research to which aptamers cannot be applied. Some areas of their expanding usages include therapeutics (Rusconi et al. 2002; Farokhzad et al. 2006; Ng et al. 2006), purification and separation (Romig et al. 1999; Michaud et al. 2003; Murphy et al. 2003), disease diagnosis and biomarker discovery (Gold et al. 2010; Ostroff et al. 2010; Turner et al. 2011), cancer imaging and detection (Martin et al. 2011; Shigdar et al. 2011; Kim et al. 2012), and target validation (Fredriksson et al. 2002; Centi et al. 2007; Xiang and Lu 2011). Many reviews have been published recently that discuss the various applications of aptamers, including their usages as potential drugs for clinical applications (Proske et al. 2005; Keefe et al. 2010), molecular imaging probes for cancer detection (Liu et al. 2013; Kadioglu et al. 2015; Ma et al. 2015), capture agents for environmental and food safety monitoring (Amaya-Gonzalez et al. 2013; Wu et al. 2014) and as vehicles for targeted-drug delivery (Tombelli et al. 2007; Cao et al. 2009; Knop et al. 2010). 


\subsection{Generation of aptamers using SELEX technique}

Aptamers are generated using an in vitro process commonly known as SELEX which is a procedure where target-binding oligonucleotides are selected from a random sequence pool of oligonucleotides through iterative cycles of affinity separation and amplification (Tuerk and Gold 1990). The SELEX process can be divided into the following categories namely: [i] designing an aptamer library (pool); [ii] performing the SELEX cycle; [iii] monitoring the SELEX progression; [iv] cloning and sequencing the selected sequences and; [v] aptamer binding property characterisation. These categories are generally applicable to most aptamer selections.

\subsubsection{Designing an aptamer library}

SELEX process begins with the generation of a randomised oligonucleotide (ssDNA or RNA) sequence library, which is normally composed of $\sim 10^{15}$ different sequences that theoretically can recognise any target molecules (Ellington and Szostak 1990; Tuerk and Gold 1990). An aptamer library is chemically synthesised by multiple couplings of adenine $(A)$, thymine $(T)$, guanine $(G)$ and cytosine $(C)$ through phosphoramidite chemistry (Ho et al. 1996). Most libraries are designed to be around 70 to 120 bases in length with a randomised core region of 20 to 80 random bases flanked by customdesigned constant-primer binding regions (17-21 bases) at 5' and 3' ends (Figure 1.4) to allow for amplification using polymerase chain reaction (PCR) (Ma et al. 2015).

Figure 1.4: A schematic diagram of an ssDNA aptamer library

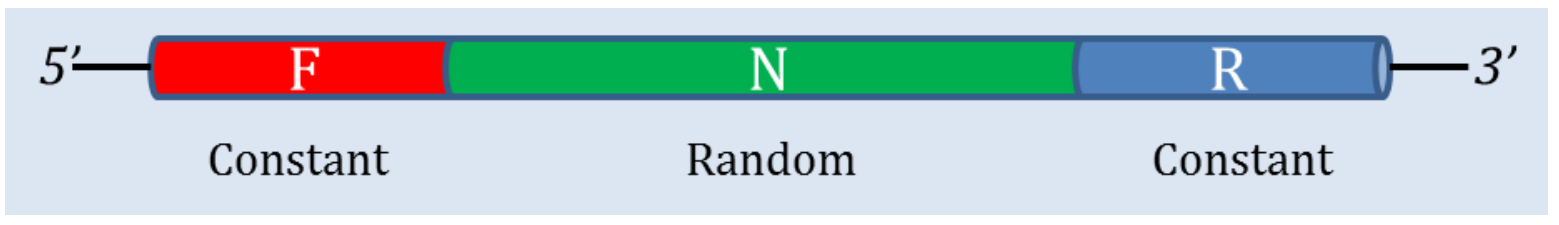

$\mathrm{F}$ and $\mathrm{R}$ represent the constant sequence regions, i.e. the forward and reverse primer binding regions respectively; $\mathrm{N}$ represents the random sequence region and; 5' and 3' refer to the terminal ends. 
When designing an aptamer library, several key parameters need to be considered; such as the length of the random core region, the size of the target molecule, and the degree of randomisation. The diversity within the library is determined by the length of the random core region. Generally, as the length of the random region increases, the structural diversity within the library also increases. This increase in diversity allows for the presence of more complex 3D structures, which are more likely to be high-affinity moieties (Luo et al. 2010). Another important parameter to consider is the size of the target molecule. The molecular size difference between proteins such as bovine serum albumin $(\sim 66 \mathrm{KDa})$ and small molecules such as TCS $(\sim 0.29 \mathrm{KDa})$ is vast. Therefore, the surface area available for an aptamer to interact with the target varies significantly between different classes of molecules. An appropriate length of the random region should strike a balance between the structural diversity required for selecting high affinity aptamers and an appropriate surface coverage of the target molecule (Marshall and Ellington 2000). Increasingly, the design of an aptamer library is driven by the structural traits of its cognate target. This may incorporate the application of computational analyses of aptamer-target interactions (Wang et al. 2009). Furthermore, when designing an aptamer library, the random core region can be either a complete randomisation or a partial (doped) randomisation (Santosh and Yadava 2014). To select aptamers against totally new targets, a completely randomised aptamer library is required. However, there may be some aptamers obtained previously that have already shown some affinity or specificity to their targets. Based on the critical motif whose structure is responsible for binding with the target molecule, a doped aptamer library can be synthesised to re-select optimal aptamers, which can usually improve their affinity to the target (Duclair et al. 2015; Lennarz et al. 2015). Finally, an aptamer library in-solution normally forms energy efficient secondary structures by self-folding and this may obstruct the availability of nucleotide bases to interact with the target molecule (Fabrizio et al. 2003). To overcome this, the aptamer library at the start of every SELEX round is normally heat-denatured (Jayasena 1999; Kim and Gu 2014). Following this heatdenaturing process, the linearised oligonucleotides are able to interact more freely with the target molecule. 


\subsubsection{Performing the SELEX cycle}

SELEX cycle usually involves five major steps of [i] co-incubation of an aptamer library with the target of interest to form aptamer-target complexes, [ii] partition of unbound sequences from the aptamer-target complexe, [iii] elution of the bound aptamers from the complexe, [iv] amplification of the eluted aptamers and [v] preparation of an enriched ssDNA aptamer library for the subsequent cycle of selection (Figure 1.5).

Figure 1.5: General scheme of the SELEX procedure

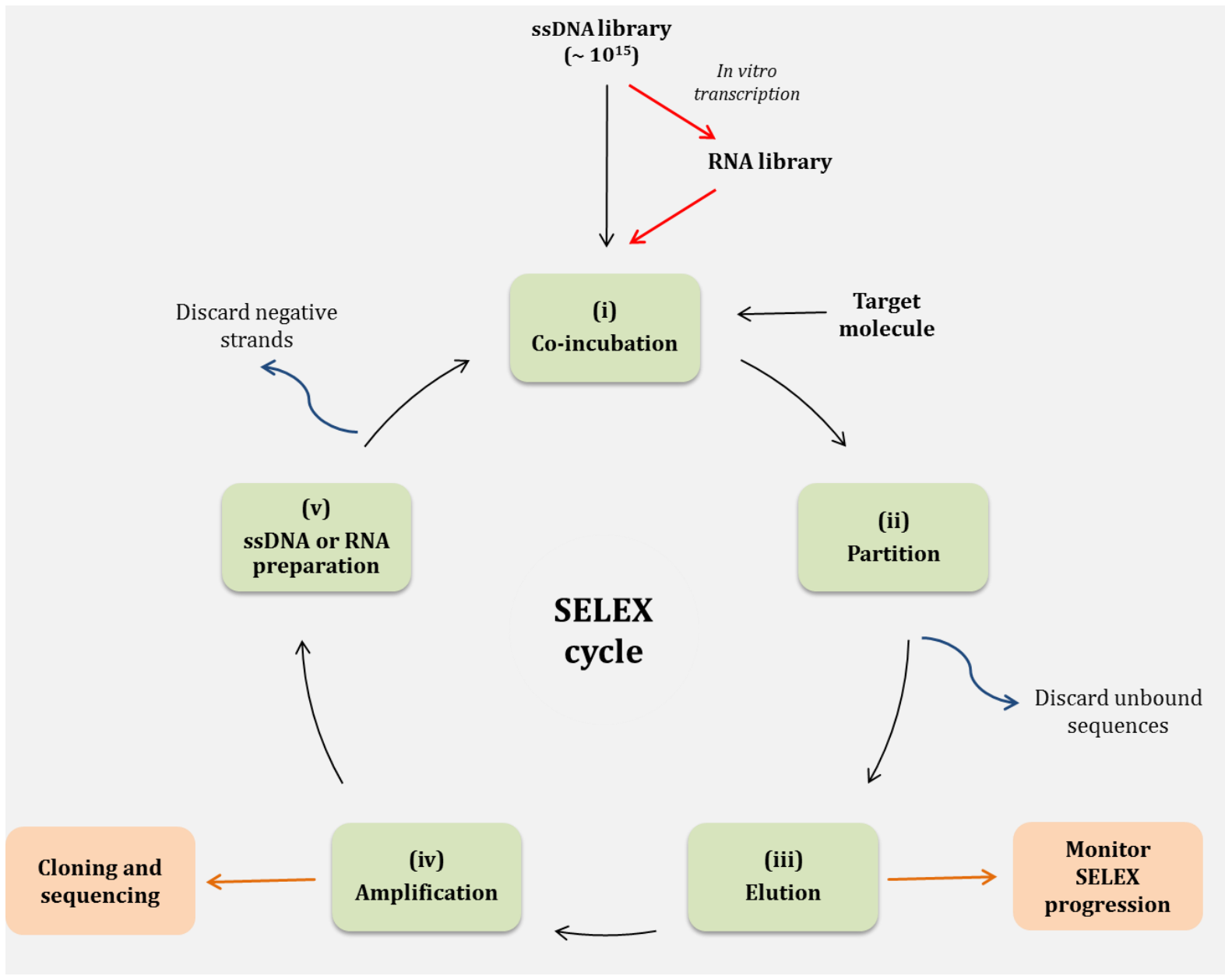

As shown in Figure 1.5, for ssDNA aptamer selection, once an ssDNA library is synthetically generated, the library is incubated with the target molecule either in a free form (i.e. in solution) or in a form that is immobilised on a solid substrate surface. Some sequences in the library will bind to the target. The unbound sequences in the library are then separated from the bound ones. 
Thereafter, the bound sequences are eluted and amplified by PCR. The amplified products are then purified and strand-separated to generate a new ssDNA library for the subsequent SELEX cycle. For RNA aptamer selection, the SELEX procedure is similar to the ssDNA aptamer selection, except that the initial ssDNA library is PCR amplified and than the PCR products are transformed into an RNA library via in vitro transcription before starting the RNA aptamer selection. During selection, the selected RNA sequences from each round are amplified by reverse transcription PCR (rt-PCR). The rt-PCR products are then transcribed in vitro again to generate a new RNA library for the subsequent round of selection.

\subsubsection{Co-incubation}

For the first step of the SELEX cycle, a synthetic aptamer library and the target of interest are co-incubated to form aptamer-target complexes in an appropriate buffering system at optimal binding conditions. An interaction between an aptamer and the target molecule induces a conformational change within the aptamer, which forms the most energy efficient 3D structure specific to the target (Foerster et al. 2012). This induced-fit mechanism can be influenced by the ionic composition, i.e. sodium $\left(\mathrm{Na}^{+}\right)$, magnesium $\left(\mathrm{Mg}^{2+}\right)$, calcium $\left(\mathrm{Ca}^{2+}\right)$ and potassium $\left(\mathrm{K}^{+}\right)$ions, as well as physicochemical properties, i.e. temperature, $\mathrm{pH}$ and ionic strength, of the buffering system.

The buffer composition can have a profound influence on the overall efficacy, function and structural conformation of aptamers (Baaske et al. 2010). Nucleic acids are polyanionic molecules and negative-negative charge repulsion may inhibit the formation of complex structures and thus impede binding with the target without the presence of counter ions. In particular, the monovalent ion $\mathrm{Na}^{+}$is effective for neutralising negative charges of the phosphate molecules on the DNA backbone, thereby preventing the negative-negative charge repulsion. The divalent ions $\mathrm{Mg}^{2+}$ and $\mathrm{Ca}^{2+}$ can influence the degree of DNA folding. Study by Carothers et al. demonstrated that performing SELEX under low $\mathrm{Mg}^{2+}$ concentrations (1-2.5 $\mathrm{mM}$ ) increased the stringency of selection, leading to the production of high-affinity binding aptamers (Carothers et al. 2010). 
Another study by Cruz-Aguado and Penner demonstrated the binding affinity of an ochratoxin A (OTA) aptamer increased when $\mathrm{Ca}^{2+}$ ions were present (Cruz-Aguado and Penner 2008). Both studies suggested that aptamers form a target binding complex in coordination with divalent ions. Many aptamers such as thrombin-binding (Paborsky et al. 1993), hematoporphyrin (Okazawa et al. 2000), HIV-1 integrase (de Soultrait et al. 2002), and adenosine triphosphate (ATP) aptamers (Huizenga and Szostak 1995) fold into G-quadruplex structures. During the generation of these aptamers, $\mathrm{K}^{+}$was included in the selection buffers, since $\mathrm{K}^{+}$is required to help stabilise $\mathrm{G}$-rich sequences to fold into the G-quadruplexes.

Both ssDNA and RNA aptamers require nuclease-free water for buffer preparation to prevent degradation by nucleases (Williams et al. 1997). However, ssDNA aptamers are less susceptible than RNA aptamers to nucleases, and thus they are applied more widely for environmental sensing applications (Kim et al. 2007; Song et al. 2008; Xu et al. 2012). Other factors, such as temperature, $\mathrm{pH}$ and ionic strength also affect the binding ability and efficiency of aptamers to form the most favoured binding complexes during selection (Hianik et al. 2007). Determining the appropriate in vitro conditions is guided primarily by aptamer endpoint applications. Moreover, it is generally recommended that these conditions remain constant throughout the selection process so optimal binding efficiency can be achieved.

Surfactants such as sodium dodecyl sulphate (SDS), Tween 20 to 80 and Nonidet P40 have been used widely in biological assays for reducing non-specific bindings, coating solid surfaces and adjusting overall charge distributions (Dias et al. 2000; Dias et al. 2004). For aptamer selection, surfactants are often included in the selection buffers to reduce nonspecific interactions (Lin and McNatty 2009). Manipulating the amount of surfactant used $(\sim 0.01-0.1 \%, w / v)$ can often improve the pooling of highly stable and avid aptamertarget complexes, thereby eliminating aptamers with weak associations (Kumar 2014). 


\subsubsection{Partition}

Following the formation of aptamer-target complexes, separation of bound sequences from unbound or weakly bound sequences is one of the most crucial steps during selection, as this enables the screening of high affinity aptamers from the library pool. The most commonly used partitioning method for protein targets is nitrocellulose membrane filtration owing to the fact that most proteins can passively be adsorbed onto the membrane surface (Bianchini et al. 2001). The first SELEX report utilised this partitioning method to separate binding and nonbinding RNA aptamers for T4 DNA polymerase (Tuerk and Gold 1990). Protein with bound RNA molecules was retained by the membrane, whilst the unbound RNA molecules was washed off. This method of partitioning is well studied and is still being used today (Gopinath et al. 2006; Sekiya et al. 2006).

Alternatively, size discrimination techniques such as gel and capillary electrophoresis have been used successfully to separate aptamer-target complexes from unbound sequences. Electrophoretic technique exploits the difference in mobility of an analyte when an electrical field is applied. The change in mobility is usually the result of the size and charge ratio of the free analyte and analyte-bound complexes as they passes through either a slab gel or capillaries. The binding of aptamers to large molecules demonstrate a significant size difference between bound and unbound sequences when compared with binding to small molecules, thus the electrophoresis technique is an ideal separation method for selecting aptamers binding to large molecules. Electrophoresis-based partition methods have been used to identify aptamers targeting a myriad of proteins, such as IgE (Mendonsa and Bowser 2004), neuropeptide Y (Mendonsa and Bowser 2005), and Muts protein (Drabovich et al. 2005).

For small molecules, size discrimination techniques are not applicable as the change in overall size of aptamers after they form binding complexes with small molecules is relatively small. Therefore, when developing aptamers against small molecules, the target of interest is often immobilised onto the surface of a solid matrix, such as sepharose, agarose or magnetic beads (Ciesiolka et al. 1995; Tombelli et al. 2005; Stoltenburg 
et al. 2005), thereby allowing easy partitioning of unwanted sequences through multiple washing steps. However, the fixation of the target molecule onto the surface of a matrix may introduce non-specific binding sites to be recognised by aptamers and/or result in structural changes of the target molecule. The SELEX technique overcomes this problem by performing multiple rounds of negative selection using unconjugated matrix (Jayasena 1999), and counter selection using structurally similar molecule-immobilised matrices (Jenison et al. 1994) to eliminate sequences displaying affinity for either the solidsupport or compounds sharing structural similarity to the target.

Apart from these conventional techniques, more advanced partitioning methods have been reported for aptamer selection, such as the use of affinity tags (Dobbelstein and Shenk 1995), centrifugation (Rhie et al. 2003), flow cytometry (Davis and Szostak 2002), and electrophoretic mobility shift assays (Tsai and Reed 1998). Several of these partitioning methods for aptamer selection have been reviewed in detail by Gopinath (Gopinath 2007).

\subsubsection{Elution}

The next step in the SELEX cycle is to elute bound aptamers from aptamer-target complexes. Interactions between the bound sequences and the target of interest are noncovalent in nature, and so several methods are possible for eluting these sequences. Elution by affinity with free target molecule is the preferred method (Bridonneau et al. 1999; Silverman 2009). In this procedure, the free target molecule in solution competes for aptamer binding with the target molecule affixed on a matrix. Typically, the target molecule used for elution should be as pure as possible, and concentrations required for a successful elution are often at milimolar (mM) levels. Studies by Huizenga et al. and Cruz-Aguado \& Penner have demonstrated the use of 3 mM ATP and 2 mM OTA to elute ATP and OTA aptamers respectively (Huizenga et al. 1993; Cruz-Aguado and Penner 2008). 
It is worth noting that the target-based elution method is restricted by the solubility of the target molecule. For some EDCs, such as TCS, bisphenol A (BPA) and OE2, their aqueous solubilities are at low $\mu \mathrm{M}$ levels, thus it is not usually feasible to prepare these molecules at the concentrations required for competitive elution. Furthermore, the cost related with the use of target-based elution can also be a limiting factor. A study by McKeague et al. demonstrated the use of free fumonisin B1 (FB1) to elute FB1 binding aptamers could cost up to $\$ 12,000$ if the FB1 is obtained through a standard chemical supply house (McKeague et al. 2010). Other commonly used methods for elution include: changes in ionic strength or the $\mathrm{pH}$ of the buffer, thermal displacement by heating (Bruno and Kiel 2002), and the use of denaturing substances such SDS, ethylenediamine tetraacetic acid (EDTA) and urea, to disrupt hydrophobic stacking of DNA bases or chelation of aptamer-target complexes (Geiger et al. 1996; Bridonneau et al. 1999; Bianchini et al. 2001). It is also worth noting that complete elution of bound sequences may not always be achievable. Some sequences possessing extremely high affinity to the target molecule are often difficult to elute, and therefore are lost during elution (Liu and Stormo 2005; Tombelli et al. 2005).

An alternative strategy for obtaining bound sequences is to use aptamer-target complexes directly as the template for PCR amplification, providing the binding complexes do not interfere with the Taq polymerase used in PCR. This strategy has been applied successfully in generating OE2-specific aptamers (Kumar 2014). In this approach, the bound OE2 aptamers dissociates from the aptamer-bound matrix during the denaturation step of the PCR cycle, and become freely available for primers to bind, thereby initiating the amplification process (Kumar 2014).

\subsubsection{Amplification}

Once separated from the target molecule, the isolated-sequences are amplified to yield an enriched-amount of selected aptamers. The amplification process is typically achieved by performing PCR for ssDNA aptamer selection or rt-PCR for RNA aptamer selection. 
These conventional techniques are, in general, effective in amplifying the isolated sequences after elution. However, this is not always true due to a plethora of diverse sequences present in the recovered pool. When using these randomised sequences as templates, the conventional PCR amplification is usually inefficient and prone to the production of non-specific by-products rather than desired aptamer sequences (Tolle et al. 2014). Although optimising PCR conditions, such as primer concentration, annealing temperature, and the number of amplification cycles can, to some extent, reduce the amount of PCR by-product formation, it is not always effective. Thus, inefficient PCR amplification is a key limitation to a successful SELEX selection. To overcome this problem, some advanced PCR techniques, such as real-time PCR and emulsion PCR have been incorporated to the SELEX procedure for aptamer selection (Wang et al. 2005; Huenniger et al. 2014; Yufa et al. 2015).

Real-time PCR is preferable over standard PCR as the optimal numbers of PCR cycles necessary to amplify the isolated sequences can be followed in real time by introducing a fluorescent dye such as SYBR green; this dye preferentially fluoresces when binding to double-stranded DNA (dsDNA) molecules as the reaction progresses (Wang et al. 2005). The graphic output of a real-time PCR can be used to determine when the amplification should be stopped. This is important as over amplification can often result in the formation of PCR by-products, thus reduce the efficiency of aptamer enrichment (Tolle et al. 2014). Emulsion PCR is another advanced PCR technique, in which the diverse aptamer sequences can be diffused to form single-sequence templates, so that each single-sequence template is specifically amplified thereby reducing the formation of PCR by-products. For example, by combining emulsion PCR amplification and capillary electrophoresis, Yufa et al. successfully developed high-affinity aptamers against AlkB homologue 2, in three rounds of selection, while SELEX with conventional PCR failed to produce the aptamer (Yufa et al. 2015). With the aim of reducing PCR by-products, some other PCR techniques, such as rolling circle PCR (Zhou et al. 2007) and high-fidelity digital PCR (Ouellet et al. 2015) have also been applied in the SELEX procedure. 
Regardless of which PCR method is chosen for aptamer discovery, designing a functional pair of primers is essential, as it determines the efficiency of PCR amplification. One consideration in primer design is nucleotide length, which is typically around 17-21 nucleotides. This length is efficient to specifically recognise the primer region of an aptamer library. Also, GC content must be carefully controlled in order to maintain a desirable melting temperature (Tm), which is approximately $\pm 5^{\circ} \mathrm{C}$ of the annealing temperature used. Primers are designed to have similar $\operatorname{Tm}$ (within $1^{\circ} \mathrm{C}$ ), and to avoid stretches of repeated bases (such as AAAAAA or CGCGCG) in order to encourage the proper primer annealing positions. Furthermore, primers should not demonstrate significant secondary structure formation (hairpins) near the Tm, or form either homoor hetero-dimers. These interactions will effectively lower the yield of PCR by decreasing the amount of primers available for annealing to the template strand.

Introducing mutations into PCR amplicons is another consideration that is important in the SELEX process, as it ultimately determines the overall diversity of aptamer libraries towards the target molecule (Jenison et al. 1994; Kim and Gu 2014). Mutations within the random region of aptamer sequences enable the production of variants that potentially enhance their binding properties through iterative cycles of selection (Lin and McNatty 2009). Mutation can be introduced by manipulating PCR conditions by: [i] varying the concentrations of individual nucleotides (dNTPs); [ii] using nucleic acid analogues such as deoxy inositol triphosphate (dITPs) and; [iii] using a DNA polymerase with low fidelity. Among these approaches, alterations to the nucleotide composition in the dNTP mix during amplification can introduce bias of nucleotides within the aptamer library (Hamula et al. 2006), whereas substitutions of dNTPs with dITPs can often lead to the biased incorporation of $C$ and G over A and T nucleotides (Kuipers 1996). Therefore, introducing mutations using low fidelity DNA polymerase is becoming increasingly popular, as a high rate of mutation can be achieved through the manipulation of $\mathrm{Mg}^{2+}$ concentration in the reaction buffer or substitution of the $\mathrm{Mg}^{2+}$ with manganese ions $\left(\mathrm{Mn}^{2+}\right)$ (McKeague et al. 2010). 


\subsubsection{Preparation of ssDNA aptamer library}

PCR amplification results in the formation of dsDNA molecules, whereas aptamer selection is usually carried out using only the positive strands of the dsDNA. Therefore, the positive strands need to be separated to start the next cycle of selection. The most commonly used technique for ssDNA preparation is alkaline denaturation utilising the natural interaction between biotin and streptavidin (Murphy et al. 2003). In this approach, a biotin tag is incorporated into the dsDNA molecules using biotinylatedprimers during amplification. Streptavidin-coated affinity matrices (e.g. agarose or magnetic beads) are then used to bind the biotinylated-strands and the unlabeled complementary strands can be eluted using an alkaline solution. The ssDNA aptamers isolated after alkaline denaturation are immediately neutralised using an acid solution to prevent degradation.

Other general techniques for ssDNA preparation include: [i] asymmetric PCR amplification, where the replication initiated by one PCR primer is relatively inefficient, leading to the accumulation of ssDNA molecules synthesised from the other primer (Ellington and Szostak 1992); [ii] the use of a hexaethyleneglycol (HEGL) spacer and an extension of several adenine nucleotides (polyA) at the 5 '-end of the reverse primer. The HEGL-spacer acts as a terminator for DNA polymerase. The elongation of positive strands stops, whilst negative strands grow further. The two strands can then be separated by size using electrophoresis (Williams and Bartel 1995) and; [iii] the use of phage lambda exonuclease to cleave the phosphorylated strands of dsDNA molecules (Fitter and James 2005). Avci-Adali et al. have provided a detailed review of the commonly used methods for ssDNA preparation (Avci-Adali et al. 2010).

Generation of ssDNA is the last step of a SELEX cycle. The resulting ssDNA molecules are then subjected to the subsequent SELEX cycle that serves to either increase their affinity for the target molecule (positive selection) or eliminate sequences that have affinity for undesirable compounds (negative or counter selections). Typically, the SELEX cycle is repeated for 6-20 rounds in order to generate and isolate target specific aptamers with high binding affinities (Gopinath 2007). 


\subsubsection{Monitoring the SELEX progression}

The evolution of aptamers with increasing affinity towards a target molecule is progressively monitored throughout selection to determine if SELEX conditions are appropriate and also to establish when the polyclonal aptamer library has achieved sufficient sensitivity to the target molecule. Techniques used for assessing sensitivity are normally associated with aptamer partitioning methods. In a nitrocellulose membranebased SELEX system, dot blotting (or Eastern blotting) is an easy and convenient method for characterising aptamer affinity from each round of selection. Based on the dot blotting method, target molecules, either proteins or small molecules, are dissolved in an appropriate solvent and spotted onto a membrane surface or a surface capable of retaining the target molecules. The membrane is then exposed to the isolated aptamers that are modified with a signaling molecule such as a fluorophore or a biotin. Subsequently, the aptamer-target binding event is visualised by fluorescence, luminescence or chemiluminescence (Navani et al. 2009). Dot blotting has been successfully applied to the SELEX technology for identifying ovine follicle-stimulating hormone (Lin and McNatty 2009), OE2 and BPA-specific aptamers (Kumar 2014).

In an affinity column-based SELEX procedure, affinity column elution is the preferred method for monitoring SELEX performance (Ciesiolka et al. 1995; Stoltenburg et al. 2005; Tombelli et al. 2005). This can be achieved by labeling aptamers with either a radioisotope or fluorescent tag. After the labelled aptamers have bound to the column matrix, the attached aptamers can then be eluted and quantified by either using a radioisotope counter or a fluorescent reader. Subsequently, the amount of aptamers recovered is compared to the amount of aptamers added before binding to the matrix. Thereafter, the percentage of aptamers recovered after progressive cycles of selection can be monitored. An increase in the percentage of aptamers recovered when compared to the initial library indicates that more of the aptamers are binding to the target, resulting in an increasingly enriched pool. Typically, less than $5 \%$ of recovery is observed for the first few rounds of selection because the aptamer pools from earlier rounds are generally not enriched enough (Stoltenburg et al. 2005). 
However, as the selection progresses past the initial rounds, one usually observes that the pool of aptamers gradually increases with respect to the efficiency of recovery, indicating that selection conditions are appropriate for enriching the pool. The selection process is considered complete when the following conditions are met: [i] there is a significant increase in the aptamer recovery between the initial library and the selected library and; [ii] further rounds of selection do not increase the aptamer recovery. Once these criterias are met, the recovered aptamers are ready for cloning and sequencing to identify monoclonal aptamer sequences.

\subsubsection{Cloning and sequencing}

The SELEX process is designed so that the amount of aptamers with the highest binding affinity to the target molecule reaches a saturation level after several rounds of selection. In general, individual aptamer sequences (also known as monoclonal aptamers) can be identified from the last round of the aptamer library using DNA cloning and sequencing technology. Subsequently, the identified nucleotide sequences of each individual aptamer can be evaluated using in silico analytical tools such as Clustal Omega (Larkin et al. 2007) and Geneious (Kearse et al. 2012). These tools enable the identification of key nucleotide regions within each aptamer sequence, and the demonstration of their evolutionary relationships. The identified sequences can also be evaluated using mFold to predict their two-dimensional (2D) structures (Zuker 2003). The predicted 2D structures can be further analysed to identify key motifs that are perhaps crucial for aptamer-target interactions. Cloning and sequencing are the last steps of the SELEX cycle.

\subsubsection{Aptamer characterisation}

Following aptamer generation, [i] assessing binding affinity and specificity of the monoclonal sequences, [ii] determining $K_{d}$ values, and [iii] confirming in-solution binding capability are important steps to be undertaken for aptamer characterisation. Sequences capable of binding to the target molecule are considered to be "aptamers". Nonbinding sequences are considered to be those oligonucleotides that either bind to non-target entities or have low affinity to the target. 


\subsubsection{Assessing binding affinity and specificity}

Methods used for monitoring the progression of SELEX, such as dot blot assay and affinity column elution (see Section 1.3.3) are generally applicable for screening binding affinity and specificity of the identified aptamers. Instead of using the isolated sequences (which are polyclonal) after elution, monoclonal aptamers can be chemically synthesised and modified to perform target binding assays. Once the binding assays are completed, the binding affinity of each monoclonal aptamer is ranked, so that aptamers with low affinity can be excluded from further studies, and high affinity aptamers are then subjected to determine their $K_{d}$ values.

\subsubsection{2 $K_{d}$ determination}

In the SELEX study, $K_{d}$ is a reliable measure to evaluate the strength of aptamer-target interactions. The $K_{d}$ is a calculated value that describes the ratio of unbound and bound aptamers, expressed in molar units (M) corresponding to the concentration at which half of the saturation binding of an aptamer to the target molecule occurs. In order to determine the $K_{d}$ value, the amount of aptamer-target complexes formed over a range of starting concentrations must be measured. Generally, this is achieved when the concentration of one of the two variables (i.e. aptamer or target molecule) is kept constant while the other is serially varied. General methods used for determing the $K_{d}$ value include: [i] surface plasma resonance (Tombelli et al. 2005), [ii] aptamer-based ELISA (Woo et al. 2013), [iii] equilibrium filtration or dialysis (Koizumi and Breaker 2000; Geiger et al. 1996), and [iv] fluorescent binding assay (Mann et al. 2005).

\section{[i] Surface plasma resonance}

Surface plasma resonance (SPR) is a commonly used technique to determine kinetic parameters such as, equilibrium constants and stoichiometry of a binding reaction (Di Primo et al. 2011). When light strikes the surface of a metal (usually silver or gold), at a particular angle, it can excite electrons on the metal surface, causing an electrodensity wave to propagate along the surface. This phenomenon, known as SPR, allows for precise measurements of surface molecular interactions in real-time (Zeng et al. 2014). 
Instruments based on the SPR phenomenon can be used for label-free detection between targets immobilised at a solid-liquid interface and their binding partners in solution. In case of aptamer-target interaction, SPR has been used to determine $K_{d}$ values for aptamers targeting either proteins such as HIV-1 tat protein (Tombelli et al. 2005), vascular endothelial growth factor (Li et al. 2007), and retinol binding protein 4 (Lee et al. 2008), or small molecules such as kanamycin B (Kwon et al. 2001), codeine (Win et al. 2006), and S-adenosylhomocysteine (Gebhardt et al. 2000). Though the concept of SPR is remarkable, the instrumentation associated with the SPR technology is expensive, which restricts the use of SPR in many laboratories. Therefore, there is a niche market to develop simple, low cost and user-friendly assay for $K_{d}$ determination.

\section{[ii] Aptamer-based ELISA}

The concept for aptamer-based ELISA is to replace antibodies with aptamers for biorecognition. In most cases, this assay is performed by coating a fixed amount of protein target in a microtiter plate, and then increasing the concentrations of target specific aptamers (labelled with a biotin tag) being added to each well. Thereafter, unbound aptamers are washed away, and an enzyme, usually streptavidin-horse radish peroxidase (ST-HRP) is added to each well for binding with the aptamers. Finally, the enzyme substrate, such as $3,3^{\prime}, 5,5^{\prime}$-Tetramethylbenzidine (TMB) is added for colour development. The amount of bound aptamers can then be quantified by measuring the optical density (OD) of the colored solution in each well, so that a binding isotherm can be plotted based on the OD reading at each aptamer concentration. The aptamer-based ELISA has been utilised to determine the $K_{d}$ values for protein-specific aptamers, such as influenza virus NS1 protein (Woo et al. 2013), epidermal growth factor receptor variant III (Tan et al. 2013), and Ras protein (Wang et al. 2014). However, the assay procedure usually involves multiple binding and washing steps, which can be time consuming. In addition, the enzyme substrate, TMB, is carcinogenic and therefore not user-friendly. 


\section{[iii] Equilibrium filtration or dialysis}

Equilibrium filtration or dialysis is commonly used method to study the affinity of aptamers for small molecules. In both approaches, an aptamer and its target are mixed in a series of microfuge tubes, in which the amount of aptamer is kept constant, but the amount of target is increased incrementally. These samples are allowed to reach equilibrium. Thereafter, separation of unbound target molecules from aptamer-target complexes is performed by applying a molecular weight cut-off membrane such as a size exclusion filter or a dialysis chamber. Both methods utilise radio-isotope labelled target molecule, and the exact amount of unbound target is quantified by measuring the amount of radioactivity inside the filter or dialysis chamber using a scintillation counter. Since input concentrations of the aptamer and target analyte are known, a series of numbers that describes the amount of aptamer bound at each target concentration can be calculated to plot a binding curve for $K_{d}$ determination.

Equilibrium-based methods have been applied in many aptamer studies. Koizumi and Breaker demonstrated the use of equilibrium filtration to determine the $K d$ value for an cAMP specific aptamer using ${ }^{32} \mathrm{P}$-labelled cAMP and a centrifugal filter that had a molecular weight cut-off of 3000 Da (Koizumi and Breaker 2000). Geiger et al. used equilibrium dialysis to determine the $K_{d}$ value of an $L$-arginine specific aptamer using a dialysis chamber with a molecular weight cut-off of 5000 Da to separate $\mathrm{L}-\left(2,3,4,5-{ }^{3} \mathrm{H}\right)$ arginine at different concentrations (Geiger et al. 1996). It is worth noting that equilibrium-based methods assume free crossing of small molecules to a membrane, which may not always be the case for some molecules, such as ATP. A study by Huizenga and Szostak demonstrated an incomplete passing of ATP through the membrane (Huizenga and Szostak 1995). Moreover, both methods utilise radio-isotope labelled targets, thus a radio-isotope certified laboratory is required to undertake these assays which is not always available. 


\section{[iv] Fluorescent binding assay}

Fluorescent binding assays, first described by Mann et al. for the selection of ethanolamine-binding aptamers, have attracted a lot of attention in the aptamer field due to simplicity, low cost, and user-friendly features (Mann et al. 2005). The method has been used to determine $K_{d}$ values for aptamers targeting either proteins such as streptavidin (Stoltenburg et al. 2005), platelet derived growth factor BB (Cho et al. 2010), and anthrax protective antigen (Choi et al. 2011), or small molecules such as, chloramphenicol (Mehta et al. 2011), L-tryptophan (Yang et al. 2011), polychlorinated biphenyls (Mehta et al. 2012), and okadaic acid (Eissa et al. 2013).

The assay utilises convenient chemical synthesis and modification of oligonucleotides. By labelling an aptamer with a fluorophore, such as FAM (Bing et al. 2011), Alexa488 (Qian et al. 2009) or Cy3 (Choi et al. 2011), quantifications of aptamers can be achieved through the measurement of fluorescent intensity. The assay requires the immobilisation of a target molecule to a solid support (e.g. polystyrene microtiter plates, magnetic beads, agarose beads and sepharose beads). Then, a constant amount of the immobilised target can be incubated with a fluorophore-labelled aptamer at variable concentrations. Following incubation, any excess unbound aptamers can be removed by multiple washing steps, and bound aptamers are then eluted from aptamer-target complexes through the use of denaturing substances such as SDS, EDTA or urea. Fluorescent intensity of the eluted aptamers can then be determined by fluorometry using appropriate excitation and emission wave-lengths corresponding to the fluorophore used for aptamer labelling. Thereafter, the amount of eluted aptamers can be calculated by using fluorophore calibration plots. Saturation curves can then be generated by plotting the amount of eluted aptamers versus the amount of total incubated aptamers.

\subsubsection{Aptamer in-solution binding activity}

Aptamers are normally used to measure free targets in solution, thus the in-solution binding activity of an aptamer needs to be characterised before applying the aptamer in a sensing application. 
As mentioned earlier, aptamers exhibit specific binding ability to target molecules which is a result of conformational changes from a random coiled structure to rigid tertiary structure such as hairpin or G-quadruplex structures (Wu et al. 2010; Foerster et al. 2012). It has been reported that there is a mixture of aptamers of both random coil and rigid tertiary structures before binding to target molecules and there is an equilibrium between the two conformations (Wei et al. 2007). Therefore, assessing the level of conformational change upon an aptamer binding to its target is a useful indicator for confirming the in-solution binding activity of the aptamer.

Circular dichroism (CD) spectroscopy, an optical technique that measures the difference in the absorption of left and right circularly polarised light, is a widely used technique for estimating secondary structures and conformations adopted by nucleic acids and polypeptides. In case of nucleic acids, CD spectroscopy measures the asymmetry from the asymmetric backbone sugars and helical structures adopted by oligonucleotides. CD spectroscopy is sensitive to oligonucleotide conformation within the 180 to $320 \mathrm{~nm}$ wavelength range, and particularly powerful for monitoring structural changes resulting from target-induced binding (Fasman 1996). It was reported that upon binding to the target, an aptamer with a hairpin structure possessed a CD spectrum characterised by a positive ellipticity maximum around $280 \mathrm{~nm}$ and a negative minimum around $248 \mathrm{~nm}$ (Nakano et al. 2002). CD spectroscopy is also a powerful tool for identifying aptamers with G-quadruplex structures (Paramasivan et al. 2007). For parallel G-quadruplexes, in which all four strands are in the same orientation, CD minima and maxima are typically near 240 and $264 \mathrm{~nm}$, respectively. For antiparallel G-quadruplexes, in which strands orientation alternates, the corresponding values are typically near 265 and $295 \mathrm{~nm}$, respectively (Balagurumoorthy et al. 1992; Dapic et al. 2003).

\subsubsection{Innovation in the SELEX method}

Several strategies to innovate the SELEX technique have been reported. These include: toggle-SELEX (White et al. 2001), cell-SELEX (Mallikaratchy et al. 2007), mirror imageSELEX (Eulberg and Klussmann 2003), and epitope-specific SELEX (Lao et al. 2014). 
Toggle-SELEX alternates aptamer selection between a human clinical target and an animal disease target, creating aptamers that are specific for both. Aptamers generated from toggle-SELEX can be tested in the animal target first and then evaluated for future human treatments (White et al. 2001). Cell-SELEX is essentially SELEX but with whole cells as enrichment targets. It is often combined with the toggle-SELEX, to positively enrich aptamers against disease cells expressing a target of interest, and then negatively against normal cells without the presence of the target molecule (Mallikaratchy et al. 2007). Mirror image-SELEX utilises naturally occurring $D$-chiral oligonucleotides to perform SELEX against an $L$-chiral target. At the end of aptamer generation, the $D$-chiral groups of the identified aptamer were converted to $L$-chrial groups. As a result, the $L$ chiral aptamer is capable of recognising the $D$-chiral target. Aptamers generated from the mirror image-SELEX are termed as "spiegelmers". These are extremely resistant to nuclease degradation, but retain the function, low immunogenicity, and low toxicity of their aptamer counterparts (Eulberg and Klussmann 2003). Most SELEX processes are considered to operate within a black box in that the target binding site of an aptamers is not known in advance. To overcome this problem, Lao et al. developed an epitope-specific SELEX procedure that can select aptamers binding to a desired epitope (Lao et al. 2014). This technique makes the selection of aptamers with predicted functions possible.

Another new direction of SELEX innovation is automation and high-throughput selection. The objective of automating the development of aptamers is to integrate different molecular biological methods to perform the SELEX cycle automatically. This is clearly a very complex challenge. Nevertheless, Eulberg et al. have developed an automated SELEX procedure with high flexibility and versatility in terms of choice of reagents and buffers used for selection, as well as the stringency of selection conditions (Eulberg et al. 2005). This robotic procedure can perform two selection rounds per day with proven successful outcomes. Another form of automated SELEX integrated microfluidic selection, highthroughput sequencing, and in situ-synthesised aptamer arrays (Cho et al. 2013). This selection robot can simultaneously measure the affinity and specificity for thousands of candidate sequences in parallel. 


\subsection{Aptamer-based biosensors}

An aptamer-based biosensor, often known as an aptasensor, is a compact analytical device that is used to detect the presence of a given target analyte by incorporating an aptamer as the sensing element either integrated within or intimately associated with a physiochemical transducer surface (e.g. gold nanoparticles or quantum dots), which translates the binding event into a measurable physical signal (e.g. mass, charge, heat or light) (Hayat and Marty 2014). Principles behind the use of aptamers for biosensing purposes exploit their high target affinity, as well as target-induced conformational change (Foerster et al. 2012). The change in conformation by aptamers forms the foundation for many sensing approaches, including fluorescence (Levy et al. 2005; Choi et al. 2006; Freeman et al. 2013), colorimetric (Liu and Lu 2006), and electrochemical methods (Liu et al. 2011). This versatility makes aptamers suitable candidates for the development of simple-to-use devices for analytical applications. In theory, aptamerbased biosensors can be fabricated to respond to any target for which an aptamer exists (Wang et al. 2011; Tang et al. 2014). Examples of commonly used aptasensors include ELISA sandwich, acoustic, electrochemical, quantum dot-based fluorescent and gold nanoparticle-based colorimetric aptasensors. Each of these aptasensors is explained in detail next, except for the gold nanoparticle-based colorimetric aptasensors, as it will be addressed later in Chapter 5.

\subsubsection{ELISA sandwich aptasensors}

ELISAs have been widely used in research and diagnostic medicine (Engvall and Perlmann 1971). One approach of this assay, commonly known as a sandwich-ELISA, involves the simultaneous use of two antibodies or analyte-binding receptors to firstly capture the analyte of interest and secondly to report target detection. Following this idea, Drolet et al. developed a new detection method that is appropriately named as enzymelinked oligonucleotide assay (ELONA) (Drolet et al. 1996). 
In ELONA (Figure 1.6 A), the reporting antibody was substituted for a fluorescein-tagged aptamer specific for detecting the target of interest. Using an aptamer against human VEGF as a model, this group showed that the developed ELONA can be applied to quantify human VEGF in sera over an range of 31.25 to $8,000 \mathrm{pg} / \mathrm{mL}(\mathrm{R} 2=0.99)$, and the accuracy and specificity of the ELONA was comparable to that of the well-established ELISA (Drolet et al. 1996).

Figure 1.6: Aptamer-based sandwich assays: (A) ELONA (enzyme-linked oligonucleotide assay) and (B) ALISA (aptamer-linked immobilised sorbent assay)

A. Enzyme-linked oligonucleotide assay (ELONA)
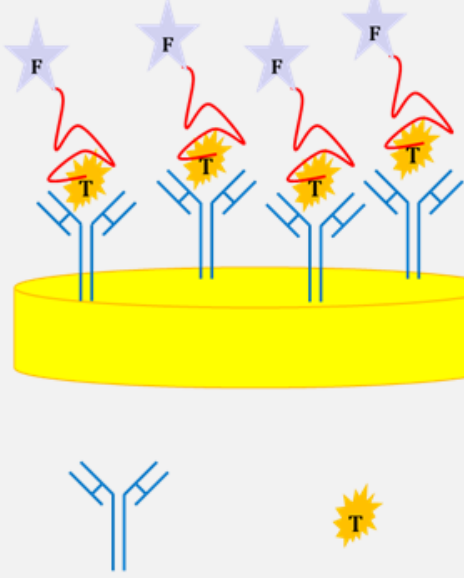

antibody

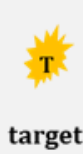

target

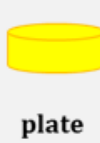

plate
B. Aptamer-linked immobilized sorbent assay (ALISA)
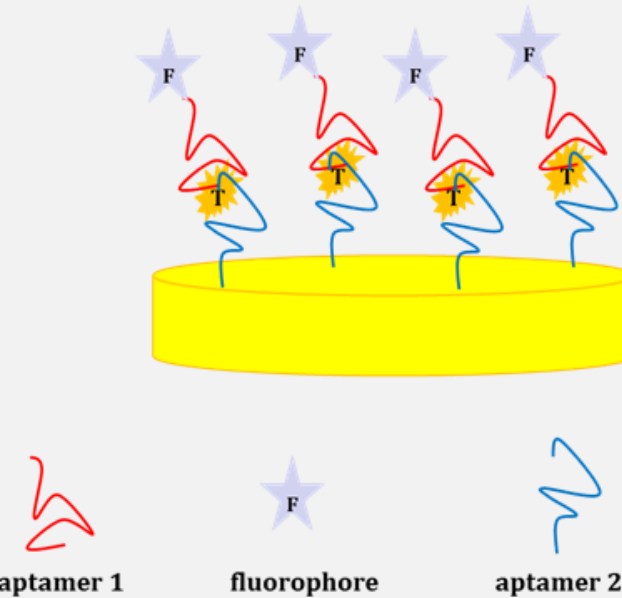

To further exclude the use of antibodies in the sandwich-ELISA, Vivekananda and Kiel devised a method that employs aptamers as both the target capturing and reporting elements (Vivekananda and Kiel 2006). This technique (Figure 1.6 B) was named as aptamer-linked immobilised sorbent assay (ALISA), and was applied by the researchers to examine the specificity of an aptamer against a bacteria surface antigen. Their results showed that the target antigen is readily detected by the ALISA at $25 \mathrm{ng} / \mathrm{mL}$ of total antigen, and the sensitivity and detection of the target is attained as low as $1.7 \times 10^{3}$ cell/mL of bacteria (Vivekananda and Kiel 2006). 
Results from both studies demonstrated that aptamers can be used in sandwich-ELISA format for the detection of specific targets. As mentioned earlier, aptamers tend to have a longer shelf-life and are easier to produce compared with antibodies. Considering that aptamers are approximately 10 times smaller than antibodies, they are also more facile to modification and labelling. These advantages render the use of the ELONA and ALISA for analyte detection even more appealing than using ELISA for similar purposes.

\subsubsection{Acoustic aptasensors}

Acoustic aptasensors utilise the change in properties of waves travelling along the surface of a signal transducer to communicate the presence of targets. In these sensors, a piezoelectric material, such as quartz crystals, is used as the signal transducer (Figure 1.7). These surfaces are frequently coated with gold and modified by coating a monolayer of self-assembled linkers to allow for the association of aptamers to the transducer surface.

Figure 1.7: General design and mechanism of an acoustic aptasensor

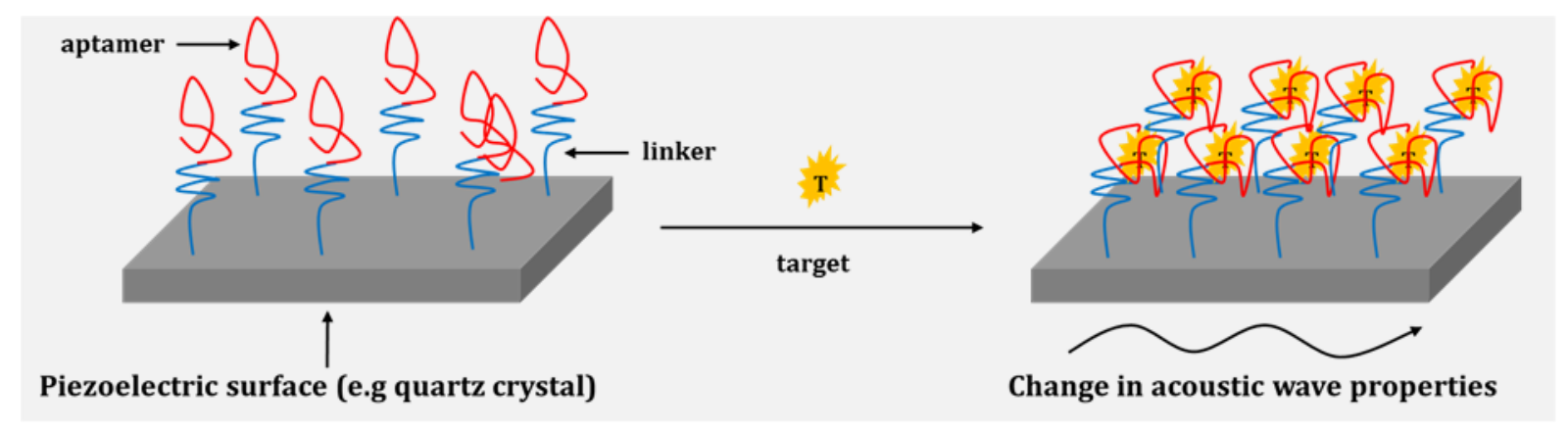

Mass loading resulting from the target binding to aptamers decreases the velocity of surface acoustic waves propagating along the transducer surface, consequently decreasing the resonance frequency of the waves or changing the phase shift between input and output waves. Thus, monitoring the change in one of these two properties would provide an indication of the absence or presence of the target molecule. 
Schlensog et al. have developed acoustic aptasensors that utilises Love waves, a form of horizontally propagating surface acoustic waves (Schlensog et al. 2004). This group coupled ssDNA aptamers against human thrombin and HIV-1 Rev proteins onto a gold layer atop a quartz crystal. The binding of thrombin or Rev proteins to their corresponding aptamers resulted in a detectable phase shift of the Love wave. The sensors developed are capable of accurately detecting their relevant target with a limit of detection (LOD) of 70 to $80 \mathrm{pg} / \mathrm{cm}^{2}$ (Schlensog et al. 2004)

\subsubsection{Electrochemical aptasensors}

Electrochemical aptasensors for molecular recognition are based mainly on the detection of electric current produced by redox reactions resulting from interactions occurring at electrode transducer surfaces (Hayat et al. 2013). Rational design of an electrochemical aptasensor usually involves: [i] choosing a transducer surface (e.g. gold and carbon surfaces); [ii] coating an aptamer onto the transducer surface (Xiao et al. 2005) and; [iii] introducing a redox label (e.g. ferrocene or methylene) to the sensor surface. This is generally performed by the use of a redox-labelled signalling oligonucleotide that has a sequence that is complimentary to the distal terminus of an aptamer, thus the labelledoligonucleotide can hybridise to the aptamer, thereby changing the electrical properties of the aptamer (Figure 1.8).

Figure 1.8: A structure-switching electrochemical aptasensor

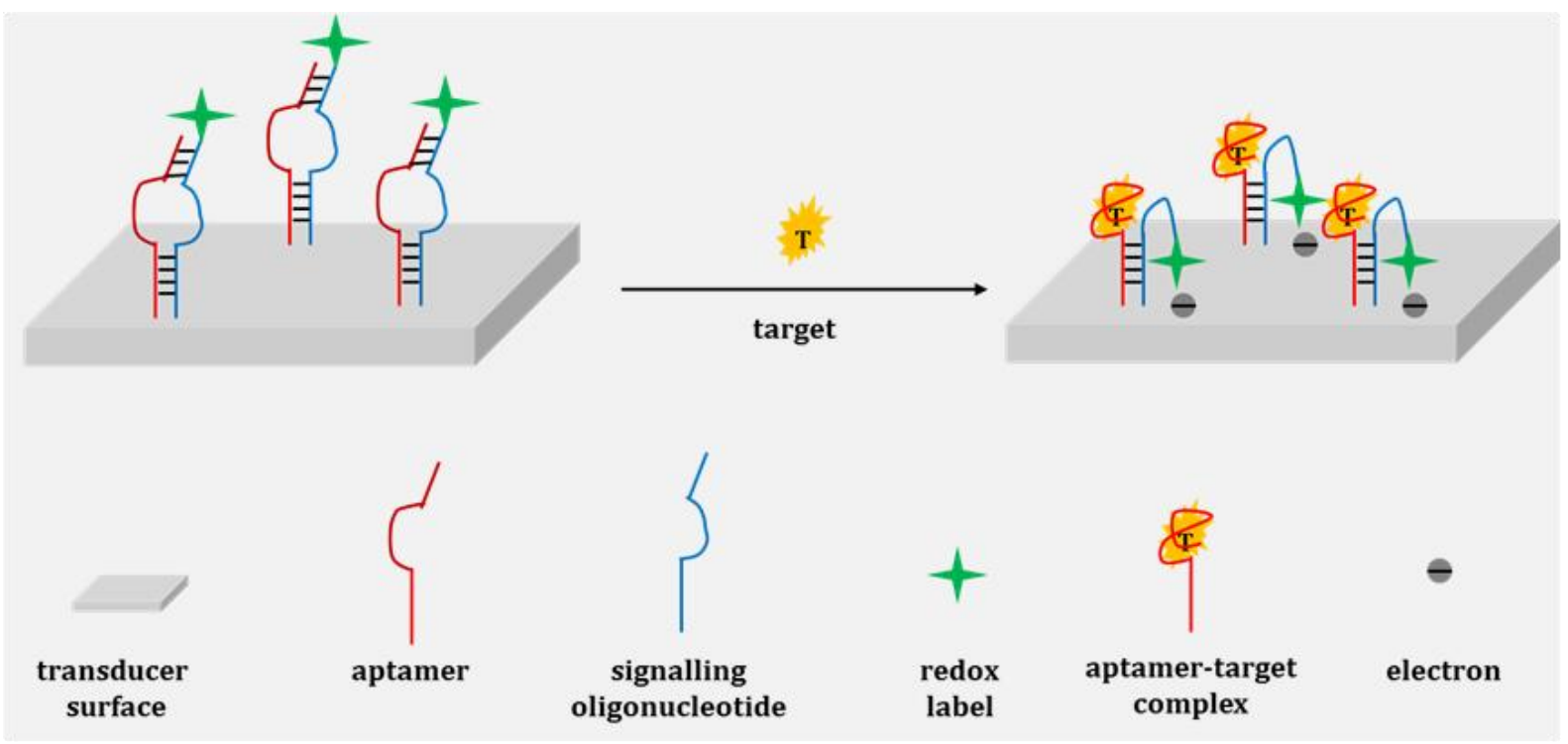


When the target is not bound to the aptamer, the two strands hybridise causing the labelled-oligonucleotide to be more rigid resulting in an increased distance between the redox molecule and the electrode, thereby preventing electron transfer. Upon binding to the target, the aptamer undergoes a series of conformational changes and releases the signalling oligonucleotide allowing it to be more flexible and accessible to the electrode surface, thereby generating a current on the electrode. Electrochemical aptasensors have been used to detect small molecules, such as cocaine in foetal calf serum or human saliva (Baker et al. 2006), OTA in wine samples (Barthelmebs et al. 2011) and okadaic acid in mussel extracts (Hayat et al. 2011), with good sensitivities and specificities.

\subsubsection{Quantum dot-based fluorescent aptasensors}

Quantum dots (QDs) are nanocrystals that are usually comprised of atoms from elemental groups II and VI (e.g. cadmium sulphide and cadmium selenide) or groups III and V (e.g. indium phosphide and indium arsenide) (Chan et al. 2002). The small radii (in the nanometer scale) of these structures leads to electron confinement causing a small and finite separation between energy levels which give rise to their unique optical properties, such as broad adsorption spectrum, narrow emission spectrum and tuneability by adjusting their size (Medintz et al. 2005). The use of QDs as a signal transducer offers numerous advantages over standard fluorescent dyes including greater photo-stability, longer fluorescent lifetimes and sharper emission bands (Medintz et al. 2005). Recent studies have demonstrated that QDs can be used in the construction of aptasensors (Levy et al. 2005; Choi et al. 2006; Huang et al. 2006; Liu et al. 2011). Levy et al. created a QD-based aptasensor (Figure 1.9), utilising fluorescence resonance energy transfer (FRET) (Levy et al. 2005), a mechanism describing energy transfer between twolight sensitive molecules referred to as a donor and acceptor (Cheng 2006). 
Figure 1.9: A quantum dot-based aptasensor utilising fluorescent resonance energy transfer (FRET)

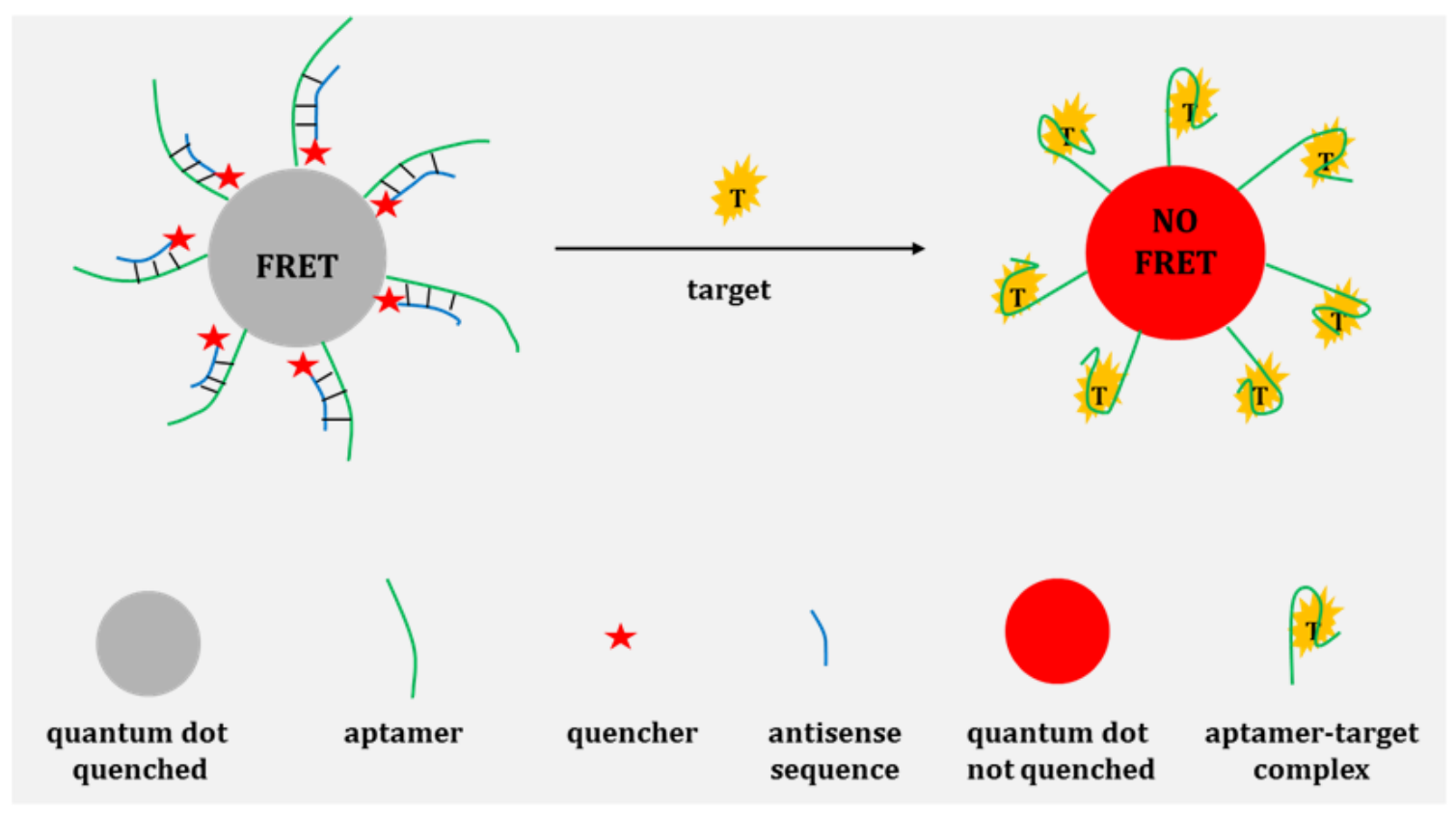

QDs used in this study are decorated with multiple thrombin-binding aptamers. These aptamers are bound by a quencher-modified antisense sequence. In the absence of thrombin, the signal emitted from the QDs is dampened due to a FRET effect from the QDs (donor) to the quencher (acceptor). Upon binding to thrombin, the conformational change of the aptamer results in the release of the quencher-modified strand, thereby switching off the FRET effect and resulting in fluorescence signal from the QDs.

A closely related method to the FRET mechanism for the development of QD-based aptasensors utilises a chemiluminescence resonance energy transfer (CRET) process. This mechanism (Figure 1.10) involves the transfer of chemically-generated chemiluminescence energy to the QDs, resulting in the photo-excitation of the QDs (Huang et al. 2006; Liu et al. 2011). 
Figure 1.10: Chemiluminescence resonance energy transfer (CRET) stimulated within the hemin and G-quadruplex coated quantum dots in the presence of luminol and $\mathrm{H}_{2} \mathrm{O}_{2}$

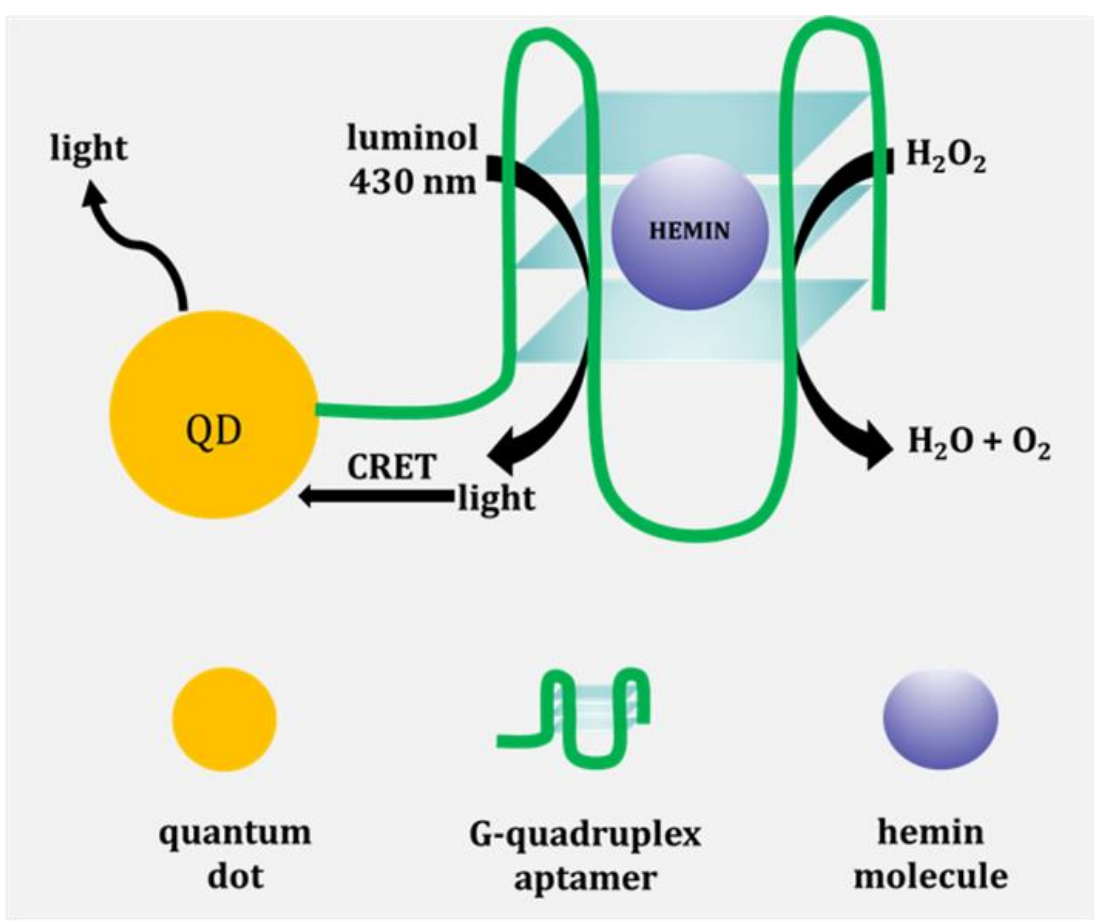

This method makes use of the fact that many aptamer sequences (e.g. thrombin-binding, hematoporphyrin, HIV-1 integrase and ATP aptamers) form G-quadruplex structures upon binding to their targets (Paborsky et al. 1993; Huizenga and Szostak 1995; Okazawa et al. 2000; de Soultrait et al. 2002). Thus, the incorporation of hemin molecules into such G-quadruplex structures can often lead to the formation of catalytically-active oligonucleotides, commonly known as DNAzymes, which are able to mimic horseradish peroxidase by catalysing the oxidation of luminol by $\mathrm{H}_{2} \mathrm{O}_{2}$, with the concomitant generation of chemiluminescence (Travascio et al. 1998; Travascio et al. 1999). Liu et al. exploited this idea by incorporating the hemin molecule into a thrombin-binding aptamer for the detection of thrombin (Liu et al. 2011). Their findings showed that the resulted DNAzyme was catalytically active and able to generate chemiluminescence on a QD surface upon the addition of the target molecule, thereby resulting in photo-excitation of the QDs by CRET effects. This method enabled sensitive detection of thrombin with a detection limit that corresponds to $1.4 \mathrm{nM}$ (Liu et al. 2011). 


\subsection{The aim and objectives}

As discussed earlier, TCS is a potential risk factor to human health because of its endocrine-disrupting properties, and also because this molecule is extensively used today. TCS is now a major concern world-wide, and recently it has been listed as an environmental contaminant by the EU Water Framework Directive (Aldous et al. 2009). In addition, Australia's industrial chemical regulatory agency declared TCS a priority existing chemical (Commonwealth of Australia 2003). The long term effects of this molecule are unknown due to the lack of suitable detecting methods to monitor the presence of TCS on a regular basis, thus its potential impact to the environment and humans remains to be evaluated. Therefore, the aim of this thesis was to develop aptamers as new capture reagents for TCS, as a first step towards the development of an alternative, user-friendly, diagnostic technique for monitoring TCS in both environmental and biological samples. With this aim, the objectives of this thesis were: [i] in vitro selection of TCS binding ssDNA aptamers; [ii] characterisation of the selected aptamers and determination of their $K_{d}$ values and; [iii] evaluating the applicability of TCS-specific aptamers in a biosensing platform. 


\section{CHAPTER 2 \\ GENERAL METHODOLOGY}

The methodology described herein refers to that for generating aptamers for TCS using an affinity column-based SELEX strategy including affinity matrix preparation and isolation, identification and characterisation of polyclonal and selected monoclonal sequences. The latter includes studies using dot blot assay for aptamer characterisation, fluorescent binding assay to determine the $K_{d}$ values and circular dichroism (CD) spectroscopy to determine potential conformational changes after binding of aptamers to TCS. Methods related to the development of a fluorescent imaging assay and gold nanoparticle-based aptasensor is described in Chapter 3 and 5 respectively. The various steps involved in the SELEX procedure are outlined in the subheadings below. All materials, reagents and suppliers are shown in Appendix I.

\subsection{Synthesis of affinity matrices for aptamer selection}

To undertake the selection of aptamers capable of binding TCS, an affinity matrix capture system was developed by conjugating TCS to sepharose beads using two conjugation strategies. Two different linker molecules 1,4-butanediol diglycidyl ether (DE) (Sundberg and Porath 1974) and epichlorohydrin (ECH) (Porath and Fornsted.N 1970) were used. TCS-matrices prepared using DE were named TCS D matrix, whereas those prepared using ECH were named TCS E matrix. To eliminate the potential of non-specific binding by aptamers to the matrix or compounds with similar chemical structures to the target, unconjugated matrices subjected to either DE or ECH chemistry, as well as BPA- and OE2conjugated matrices were also prepared. The BPA-matrix was prepared using the DE chemistry. The OE2-matrix synthesised using an amine coupling chemistry was kindly provided by Dr Shalen Kumar (Victoria University of Wellington, NZ). The following protocol describes the methods for preparing these matrices for aptamer selection. 


\subsubsection{Synthesis of TCS-conjugated matrices}

\subsubsection{Preparation of TCS solution}

A working solution containing $1.6 \mathrm{mmol}$ of TCS was prepared using $30 \mathrm{~mL}$ of $1 \mathrm{M} \mathrm{NaOH}$, and the $\mathrm{pH}$ of the solution was adjusted to $11.0 \mathrm{using} 1 \mathrm{M} \mathrm{HCl}$. The solution was filtered using a $0.22 \mu \mathrm{m}$ minispin filter to remove any precipitates formed during $\mathrm{pH}$ adjustment.

\subsubsection{Preparation of sepharose beads}

For each conjugation procedure, a $16 \mathrm{ml}$ aliquot of sepharose beads was pipetted into a sintered glass funnel and washed sequentially with $500 \mathrm{~mL}$ of $0.5 \mathrm{M} \mathrm{NaCl}, 1000 \mathrm{~mL}$ of deionised water $\left(\mathrm{dH}_{2} \mathrm{O}\right)$, and $200 \mathrm{~mL}$ of $1 \mathrm{M} \mathrm{NaOH}$. Following these washing steps, the beads were dried under a vacuum for 10 minutes (mins) before being weighed.

\subsubsection{Matrix activation}

The vacuum-dried beads were then transferred into a $50 \mathrm{~mL}$ round bottom flask. For TCS D matrix preparation, $8 \mathrm{~mL}$ of $1 \mathrm{M} \mathrm{NaOH}, 5.6 \mathrm{~mL}$ of $\mathrm{dH}_{2} \mathrm{O}$, and $2.4 \mathrm{~mL}$ of DE (13 mmol) was added for DE activation reaction and then the flask was incubated at room temperature (RT) for 8 hours (hrs) with gentle stirring.

For TCS E matrix preparation, $8 \mathrm{~mL}$ of $1 \mathrm{M} \mathrm{NaOH}, 7 \mathrm{~mL}$ of $\mathrm{dH}_{2} \mathrm{O}$, and $1 \mathrm{~mL}$ of ECH (12.8 mmol) was added for ECH activation reaction and the flask was heated at $60{ }^{\circ} \mathrm{C}$ for two hrs with gentle stirring.

Thereafter, these matrices were each placed into a sintered glass funnel and washed thoroughly using $500 \mathrm{~mL}$ of $1 \mathrm{M} \mathrm{NaOH}$. This was followed by $2000 \mathrm{~mL}$ of $\mathrm{dH}_{2} \mathrm{O}$ and then both preparations were dried under a vacuum at RT. Once completely dried, a portion (i.e. $3 \mathrm{~g}$ ) of each activated matrix was immediately blocked using $15 \mathrm{ml}$ of $1 \mathrm{M}$ ethanolamine for future use as control matrices: these are referred to as control D and E matrices. In addition to preparing control matrices, other portions of the activated beads were used for preparing either TCS D or E matrices. 


\subsubsection{TCS conjugation}

The TCS solution prepared in Section 2.1.1.1 was added to each activated matrix $(20 \mathrm{~mL}$ per matrix) and incubated overnight at RT with gentle stirring. Following overnight incubation, these matrices were washed and dried described in Section 2.1.1.3. Thereafter, any residual active groups on the matrices were blocked by overnight incubation with $15 \mathrm{~mL}$ of $1 \mathrm{M}$ ethanolamine. Following blocking, the blocked TCSconjugated and control matrices were washed with $2000 \mathrm{~mL}$ of $\mathrm{dH}_{2} \mathrm{O}$ and resuspended in $5 \%(\mathrm{v} / \mathrm{v})$ ethanol solution for long term storage at $4{ }^{\circ} \mathrm{C}$.

\subsubsection{Ultraviolet-visible (UV-visible) characterisation}

Both control and TCS-conjugated matrices (each $0.5 \mathrm{~g}$ ) were washed with $500 \mathrm{~mL}$ of $\mathrm{dH}_{2} \mathrm{O}$ and resuspended in $5 \mathrm{~mL}$ of $\mathrm{dH}_{2} \mathrm{O}$. Each matrix suspension was loaded into a $1 \mathrm{~mm}$ thick quartz cuvette. The presence of TCS molecule on the conjugated matrices was determined by scanning each matrix suspension over a wavelength range of 200-800 $\mathrm{nm}$ using a CARY 500 SCAN UV-visible spectrophotometer (Agilent Technologies, Santa Clara, California, USA). The presence of an absorption peak at $\sim 280 \mathrm{~nm}$ indicated the successful conjugation of TCS onto the surface of sepharose beads.

\subsubsection{Synthesis of BPA- and OE2-conjugated matrices}

\subsubsection{Synthesis of BPA-matrix}

BPA-matrix was prepared as followed: epoxy activated (via DE) sepharose beads were washed and dried (see Section 2.1.1.3). The activated beads were then transferred into a $50 \mathrm{~mL}$ round bottom flask. Subsequently, $20 \mathrm{~mL}$ of BPA solution ( $0.8 \mathrm{mmol}$ ) prepared in $1 \mathrm{M} \mathrm{NaOH}$ was added for conjugation. The resulting mixture was then incubated overnight at RT with gentle stirring. Following incubation, the BPA-matrix was washed, dried and blocked (see Section 2.1.1.4). Thereafter, UV-visible characterisation of the BPA-matrix was carried as described for TCS-conjugated matrices (see Section 2.1.1.5). Successful conjugation was confirmed by the presence of absorbance peaks at $\sim 225$ and $\sim 280 \mathrm{~nm}$. 


\subsubsection{Synthesis of OE2-matrix}

OE2-matrix was prepared and characterised by Dr Shalen Kumar. In brief, 17 $\beta$ oestradiol-17-hemisuccinate (OE2) was prepared in $5 \mathrm{mg} / \mathrm{mL}$ in $50 \%(\mathrm{v} / \mathrm{v})$ of 1,4dioxane in $\mathrm{dH}_{2} \mathrm{O}$, and the $\mathrm{pH}$ was adjusted to between 4 and 5 using $0.1 \mathrm{M} \mathrm{HCl}$. Then, $8 \mathrm{~mL}$ of 1,6-diaminohexane-agarose matrix was pipetted into a sintered glass funnel and washed with $100 \mathrm{~mL}$ of $0.9 \%(\mathrm{w} / \mathrm{v}) \mathrm{NaCl}$ followed by an equal volume of $\mathrm{dH}_{2} \mathrm{O}$ before adjusting the $\mathrm{pH}$ of the matrix suspension to 5.0 using $0.1 \mathrm{M} \mathrm{HCl}$. Subsequently, the OE2 solution was added to the matrix suspension and mixed by rotating the flask for 5 mins. $\mathrm{N}$-hydroxysuccinimide was added at a final concentration of $1 \mathrm{mM}$ to catalyse the reaction. Thereafter, 1-ethyl-3-(3-dimethyl-aminopropyl)-carbodiimide was added at a final concentration of $0.1 \mathrm{M}$ for conjugation reaction. The $\mathrm{pH}$ of the suspension was maintained between 5 and 6 for $2 \mathrm{hrs}$ using either $0.1 \mathrm{M} \mathrm{HCl}$ or $0.1 \mathrm{M} \mathrm{NaOH}$. Thereafter, the suspension was incubated overnight at RT with gentle stirring. Following incubation, the matrix was washed to remove any traces of un-reacted reagents using $100 \mathrm{~mL}$ of $50 \%$ (v/v) 1,4-dioxane in $\mathrm{dH} 2 \mathrm{O}$, followed by $500 \mathrm{~mL}$ of $\mathrm{dH}_{2} \mathrm{O}$. Thereafter, the matrix was resuspended in $20 \mathrm{~mL}$ of $\mathrm{dH}_{2} \mathrm{O}$. UV-visible characterisation of the OE2-matrix was carried out as stated for the BPA-matrix (see Section 2.1.2.1), and the presence of an absorption peak at $\sim 280 \mathrm{~nm}$ indicated successful conjugation of OE2 onto the surface of agarose beads.

\subsection{Preparation of ssDNA aptamer library and primers}

In this study, an N40 library consisting of 40 random nucleotides was chosen to strike a balance between structural diversity and an appropriate surface coverage of the target molecule. The N40 library (75 bases in length) contained a central random region of 40 bases, a forward and reverse region of 18 and 17 bases respectively. Forward (Pf) and reverse (Pr) primer sequences were designed by Oligo Analyzer Software provided by Integrated DNA Technologies (http://sg.idtdna.com/site). Nucleotide sequences of the N40 library and primers used in this study are summarised in Table 2.1. 
Table 2.1: Nucleotide sequences of the N40 library and primers used in the SELEX procedure

\begin{tabular}{ll}
\hline Oligonucleotide & Nucleotide sequence (5'-3') \\
\hline N40 library & ATACGAGCTTGTTCAATA-N40-TGAT AGTAAGAG CAATC-3' \\
Forward primers & ATACGAGCTTGTTCAATA \\
Reverse primers & GATTGCTCTTACTATCA \\
\hline
\end{tabular}

"N40" represents nucleic acids $\mathrm{A}, \mathrm{C}, \mathrm{T}$, and G organised in a randomised order.

For aptamer generation and characterisation purposes, a biotin tag was introduced to the 5'-end of primer sequences. Primer combinations for PCR amplification at different stages of the SELEX procedure are summarised in Table 2.2.

Table 2.2: Primer combinations used at different stages of the basic-SELEX procedure

\begin{tabular}{lll}
\hline Stage & Forward & Reverse \\
\hline Selection & Pf & biotin-Pr \\
Dot blot assay & biotin-Pf & $\mathrm{Pr}$ \\
Cloning and sequencing & Pf & $\mathrm{Pr}$ \\
\hline
\end{tabular}

$\mathrm{Pf}=$ forward primer and; $\mathrm{Pr}=$ reverse primer.

The N40 library and primers were commercially synthesised by Life Technologies (Carlsbad, California, USA). The library was synthesised on a $1 \mu \mathrm{mol}$ scale. The labelled and unlabelled primers were synthesised on a $50 \mathrm{nmol}$ scale. All synthesised oligonucleotides were purified using denaturing polyacrylamide gel electrophoresis by the manufacturer.

\subsection{SELEX preparation}

\subsubsection{Oligonucleotide hydration}

Vials containing commercially synthesised oligonucleotides received from the manufacturer were centrifuged for 30 seconds (sec) at 13, $000 \mathrm{xg}$. The vials were opened inside a Type A2 Biosafety Laminar Flow Cabinet (Labconco, Kansas City, Missouri, USA). 
An appropriate amount of DNAse free water was added to each vial to obtain a final concentration of $100 \mu \mathrm{M}$. These stock solutions were incubated at RT for 30 mins before being further diluted to make $10 \mu \mathrm{M}$ working solutions. Both the stock and working solutions were stored at $-20^{\circ} \mathrm{C}$ for future use.

\subsubsection{Preparation of selection buffers}

\subsubsection{Binding and washing buffer (BWB)}

A 10X stock solution of BWB consisting of $1000 \mathrm{mM} \mathrm{NaCl}, 50 \mathrm{mM} \mathrm{KCl}, 20 \mathrm{mM} \mathrm{MgCl} 2$, $10 \mathrm{mM} \mathrm{CaCl}_{2}$, and $200 \mathrm{mM}$ Tris- $\mathrm{HCl} \mathrm{pH} 7.5$ was prepared using $\mathrm{dH}_{2} \mathrm{O}$ and was sterilised by autoclaving. For subsequent use, the $10 \mathrm{X}$ BWB was diluted to $1 \mathrm{X}$ using $\mathrm{dH}_{2} \mathrm{O}$ and analytical grade surfactant, IGEPAL CA 630, was added to the 1X BWB to obtain a final concentration of $0.1 \%(\mathrm{v} / \mathrm{v})$ unless stated otherwise.

\subsubsection{Blocking buffer (Blotto)}

A Blotto solution was prepared fresh before immediate use by adding appropriate volume of $1 \mathrm{X}$ BWB to $99.9 \%$ fat free skim milk powder at a final concentration of $5 \%$ $(\mathrm{w} / \mathrm{v})$. The Blotto solution was vortexed until all milk powder was dissolved and then stored at $4{ }^{\circ} \mathrm{C}$ before use.

\subsection{SELEX cycle procedures}

\subsubsection{Blocking of affinity matrices}

Before each SELEX cycle, fresh aliquots of TCS D and E matrices were washed three times with $200 \mu \mathrm{L}$ of $1 \mathrm{X}$ BWB and individually resuspended in $200 \mu \mathrm{L}$ of Blotto solution for two hrs at RT to reduce the likelihood of non-specific binding by the oligonucleotides to the matrix. The amount of TCS-conjugated matrices was adjusted for each cycle of selection depending on the amount of selection pressure desired for the cycle. 


\subsubsection{Heat-denaturing of ssDNA aptamer library}

The synthetic N40 library ( $3 \mathrm{nmol}$ ) was resuspended in $200 \mu \mathrm{L} 1 \mathrm{X}$ BWB. Thereafter, the library solution was heat-denatured for 10 mins at $90{ }^{\circ} \mathrm{C}$ and then cooled at $4{ }^{\circ} \mathrm{C}$ for 15 mins before further incubation at RT for 10 mins. The heat-denaturing step was also utilised to denature aptamer libraries (each $\sim 50 \mathrm{pmol}$ ) from subsequent SELEX rounds.

\subsubsection{Co-incubation of aptamer library with affinity matrices}

Following heat-denaturing step, the aptamer library was added into each TCS-conjugated matrix solution $(100 \mu \mathrm{L}$ per matrix $)$ and the resulting solutions were then incubated at $4{ }^{\circ} \mathrm{C}$ with gentle shaking. The incubation time was adjusted for each cycle of selection depending on the amount of selection pressure required.

\subsubsection{Removal of unbound oligonucleotides}

Following incubation, each aptamer-bound matrix was transferred into a filtered syringe column. Unbound oligonucleotides were removed by thoroughly washing aptamerbound matrices using $1 \mathrm{X}$ BWB. The washing volume was increased for each cycle of selection in order to increase the selection pressure. The resulting matrices were each resuspended in $65 \mu \mathrm{L}$ of DNAse free water and were used as templates for subsequent PCR amplification.

\subsubsection{PCR amplification}

PCR was undertaken to amplify aptamer-bound matrices for selection, dot blot assay, and cloning and sequencing procedures using different primer combinations (Table 2.2). A PCR sample was prepared at a final reaction volume of $50 \mu \mathrm{L}$ using HotMaster ${ }^{\mathrm{TM}}$ Taq kit. The PCR buffer was composed of $10 \mu \mathrm{M}$ dNTPs, $1.1 \mu \mathrm{M}$ of each primer, $2.5 \mathrm{mM} \mathrm{Mg}^{2+}$, and 2 units of Taq DNA polymerase. PCR cycles were carried out using the following steps: an initial denaturing step of $95{ }^{\circ} \mathrm{C}$ for 2 mins to ensure complete denaturation of the template; followed by 35 cycles of $94{ }^{\circ} \mathrm{C}$ for $40 \mathrm{sec}$ (denaturing), $55{ }^{\circ} \mathrm{C}$ for $20 \mathrm{sec}$ (annealing), and $72{ }^{\circ} \mathrm{C}$ for $30 \sec$ (extension); and a final extension step at $72{ }^{\circ} \mathrm{C}$ for 2 mins. 


\subsubsection{Agarose gel electrophoresis}

Agarose gel electrophoresis was used to separate and analyse PCR products following amplification. The PCR products were first incubated with 1.6 $\mu \mathrm{L}$ of 100X SYBR green dye for 8 mins at RT. Then, 6X DNA loading dye was added to the products to give a final concentration of $1 \mathrm{X}$ before loading the products into the wells of a $3 \%(\mathrm{w} / \mathrm{v})$ agarose gel prepared using 1X TAE buffer. The gel was run in a Mini-Sub Cell GT Gel Tank (Bio-Rad, Hercules, California, USA) submerged in $1 \mathrm{X}$ TAE buffer for 75 mins at 95 volts. Thereafter, the gel was visualised using a UVsolo TS stand-alone gel documentation system (CORE Life Sciences, Laguna Niguel, California, USA), and the presence of 75 mer dsDNA bands which contained the desired aptamer sequences were confirmed by size comparison with a low range DNA ladder. The identified bands of interest were excised from the gel using a scalpel blade and stored at $-20^{\circ} \mathrm{C}$ until required.

\subsubsection{Extraction of dsDNA molecules}

The desired aptamer sequences were extracted from the excised gels using a Qiagen mini elute dsDNA extraction kit. The protocol used for extraction was specified in the manufacturer's guidelines with following modifications: after dissolving the excised gels in QG buffer and addition of isopropanol, the resulting solution was vortexed for $10 \mathrm{sec}$ before being centrifuged at 6,000 x g for 1 minute using mini elute columns. To maximise the retention of aptamers on the column matrix, each aliquot of the flow-through solutions was passed through 5 times before being discarded. This was followed by washing the column with $500 \mu \mathrm{L}$ buffer $Q G$ and then centrifuged at 6,000 x g for $1 \mathrm{~min}$. Thereafter, all column washing and centrifugation steps were carried out as stated in manufacturer's guidelines. The dsDNA preparations were eluted from each column membrane, using a 1:50 dilution of EB buffer. The first elution step was carried out using $50 \mu \mathrm{L}$ of EB followed by a second elution step using $30 \mu \mathrm{L}$ of EB. To stabilise the dsDNA products and to enable an improved strand separation yield, $80 \mu \mathrm{L}$ of $2 \mathrm{X}$ BWB was added to the eluate. The eluted dsDNA preparations were then stored at $-20^{\circ} \mathrm{C}$ until future use or were immediately subjected to strand separation. 


\subsubsection{Strand separation}

Alkaline denaturation of dsDNA preparations was the method of choice for generating ssDNA aptamers for either the sequential round of SELEX or an intended-dot blot assay.

\subsubsection{Strand separation to generate ssDNA aptamers for selection}

To carry out the next round of SELEX, dsDNA preparations were subjected to a strand separation process, since only forward strands are utilised during SELEX. In brief, a $30 \mu \mathrm{L}$ aliquot of streptavidin-coated magnetic beads (SMBs) was pipetted into a microcentrifuge tube before being recovered using a magnetic pipette (PickPen) with silicone tips and transferred to a microcentrifuge tube containing $50 \mu \mathrm{L}$ of $1 \mathrm{X}$ BWB. The SMBs were stirred using pipette tip for a thorough washing and then transferred to a new tube. This washing step was repeated three times, and the washed SMBs were incubated with $160 \mu \mathrm{L}$ of each biotin-labelled dsDNA preparation. The resulting solution was incubated at RT for 20 mins with periodic mixing to allow for the formation of SMB-biotin-dsDNA complexes. Following incubation, the complexes were washed with $50 \mu \mathrm{L}$ of $1 \mathrm{X}$ BWB three times, and then mixed with $30 \mu \mathrm{L}$ of $0.3 \mathrm{M} \mathrm{NaOH}$. The mixture was then incubated for 3 mins at RT, followed by removing SMB complexes (i.e. the SMB-biotin-reverse strands) using the PickPen. The leftover solution containing only the forward strands was neutralised immediately by adding $8.5 \mu \mathrm{L}$ of $1 \mathrm{M} \mathrm{HCl}$. The resulting solution containing ssDNA aptamers was used as the aptamer library for the subsequent round of selection.

\subsubsection{Strand separation to generate ssDNA aptamers for dot blot assay}

Preparation of biotin-labelled ssDNA aptamers for dot blot assay was similar to that for selection (see Section 2.4.8.1) with minor modifications. In brief, the biotin-labelled dsDNA preparations (each $160 \mu \mathrm{L}$ ) were incubated with the washed SMBs (30 $\mu \mathrm{L}$ for each preparation). The protocol for SMB washing and incubation with the biotin-labelled dsDNA molecules was performed as described previously (see Section 2.4.8.1). After formation of SMB-biotin-dsDNA complexes, strand separation was performed by adding $30 \mu \mathrm{L}$ of $0.3 \mathrm{M} \mathrm{NaOH}$, which produced the SMB bound forward strands (retained on the beads) and the unbound reverse strands (in solution). 
The forward strand-biotin-SMB complexes were then transferred to a new tube and treated with $30 \mu \mathrm{L}$ of $0.3 \mathrm{M} \mathrm{NaOH}$ for a further 1 min to ensure complete dissociation of the reverse strands from the complexes. Thereafter, the forward strand-SMB complexes

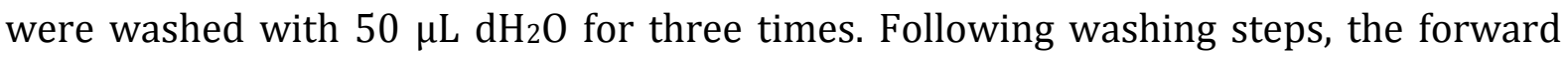
strands were dissociated from the SMB-biotin forward strand complexes by heating the complexes in an $85^{\circ} \mathrm{C}$ water bath for 2 mins to break biotin-streptavidin bonds. The SMBs were removed using the PickPen and the leftover solution containing the biotin-labelled forward strands was then used for the subsequent dot blot assay.

\subsection{Dot blot assay for monitoring SELEX progression}

TCS binding ability of polyclonal aptamers selected from the SELEX cycle was tested using dot blot assay developed in the Reproduction Laboratory (Victoria University of Wellington, NZ).

\subsubsection{Preparation of nitrocellulose membranes}

Nitrocellulose membranes for blotting the target compound were prepared by soaking the membranes 10 mins in $12 \mathrm{~mL}$ of $1 \mathrm{X}$ BWB before being completely air-dried under the laminar flow workstation. Thereafter, serial dilutions $(6.25-50 \mathrm{mg} / \mathrm{mL})$ of the target molecule prepared using analytical grade ethanol were each spotted onto the membranes at $2 \mu \mathrm{L}$ per spot. The amount of target molecules presented on the membrane was between $75-600 \mathrm{nmol}$. The ethanol was spotted $(2 \mu \mathrm{L})$ as a negative control. Serial dilutions of N-hydroxysuccinimide long chain biotin (NHS-biotin) were also spotted (2 $\mu \mathrm{L}$ per spot) at a final amount ranging from 0.1-10 nmol: these were used as positive controls to determine the consistency between dot blot assays. After spotting, the membranes were air-dried and blocked at RT using $12 \mathrm{~mL}$ of the Blotto solution. The blocked-membranes were then incubated for 2 hrs on a Cole-Parmer Rocking Shaker at 15 osc/min (Thermofisher Scientific, Waltham, Massachusetts, USA). 


\subsubsection{Incubation with biotin-labelled ssDNA aptamers}

Following blocking, biotin-labelled ssDNA aptamers (each $\sim 50$ pmol suspended in $12 \mathrm{~mL}$ of Blotto) prepared from the strand separation process (see Section 2.4.8.2) were added to sample membranes and an equal volume of Blotto solution (without any aptamers) was added to control membranes. Both membranes (i.e. sample and control) were then placed on the orbital shaker (at $15 \mathrm{osc} / \mathrm{min}$ ) for overnight incubation at $4{ }^{\circ} \mathrm{C}$. Following incubation, both membranes were rinsed three times for 10 mins with $12 \mathrm{~mL}$ of $1 \mathrm{X}$ BWB on the orbital shaker (at $40 \mathrm{osc} / \mathrm{min}$ ) to remove any unbound aptamers.

\subsubsection{Incubation with streptavidin-horse radish peroxidase}

A final conentration of $167 \mathrm{ng} / \mathrm{mL}$ ST-HRP solution was prepared using 1X BWB. Then, $12 \mathrm{~mL}$ of the ST-HRP solution was added to each membrane (sample and control). Thereafter, both membranes were incubated at RT on the orbital shaker (15 osc/min) for $2 \mathrm{hrs}$. Following incubation, each membrane was washed with $12 \mathrm{~mL}$ of $1 \mathrm{X}$ BWB for three times (each 10 mins) before being subjected to a chemiluminescence reaction.

\subsubsection{Chemiluminescence reaction}

For visualisation, $20 \mathrm{~mL}$ of chemiluminescence reaction solution $(0.1 \mathrm{M}$ Tris- $\mathrm{HCl} \mathrm{pH}$ 8.5, $25 \mu \mathrm{M}$ luminol, $396 \mu \mathrm{M}$ p-coumaric acid, and $0.01 \%$ of hydrogen peroxide in $\mathrm{dH} 20$ ) was prepared immediately before use. Both sample and control membranes were incubated together in the reaction solution for $1 \mathrm{~min}$ RT in a darkroom. The membranes then immediately placed on a clear plastic backing, wrapped using clear plastic wrap and then inserted into an x-ray cassette. X-ray film was placed on top of the membranes and exposed for 7 mins. Thereafter, the film was developed by soaking in GBX developing solution for 3 mins, and then rinsed for 1 min using $\mathrm{H}_{2} \mathrm{O}$ before a further 3 mins soaking in the GBX fixing solution prior to a final rinse in $\mathrm{H}_{2} \mathrm{O}$. The film was air dried and then evaluated to determine the binding intensity and sensitivity of the selected aptamers. 


\subsection{DNA cloning, sequencing, and nucleotide analysis}

Following dot blot assay, once the selected aptamers demonstrated successful binding to TCS, they were cloned and sequenced to identify their nucleotide compositions.

\subsubsection{DNA ligation}

To identify monoclonal forms of TCS aptamers, PCR products amplified from the final selection round using primers Pf and Pr, were purified and extracted as outlined in Section 2.4.6 and 2.4.7. Then, $3 \mu \mathrm{L}$ of each purified PCR product was added to $5 \mu \mathrm{L}$ of $2 \mathrm{X}$ ligation buffer (final concentration of $1 \mathrm{X}$ ) with the addition of $50 \mathrm{ng}$ of pGEM-T Easy cloning vector and 3 units of T4 DNA ligase in a final reaction volume of $10 \mu \mathrm{L}$. Thereafter, the reaction mixture was stirred for $30 \mathrm{sec}$ and then kept on ice for $2 \mathrm{hrs}$.

\subsubsection{Transformation and colony picking}

The ligated plasmids were transformed into chemically competent Escherichia coli (E coli) DH5- $\alpha$ cells by co-incubation on ice with the cells for 30 mins. Thereafter, the cells were subjected to "heat shock" treatment at $37^{\circ} \mathrm{C}$ for 2 mins. This was followed by a further 2 mins incubation of the cells on ice. The transformed cells were allowed to grow in $950 \mu \mathrm{L}$ of Luria broth (LB) media for $1 \mathrm{hr}$ at $37^{\circ} \mathrm{C}$ before being centrifuged at 3,000 x g for 5 mins. The resulting supernatant was discarded and the leftover pellet was then resuspended in $100 \mu \mathrm{L}$ LB media. Thereafter, the LB pellet solution was plated aseptically onto LB agar plates containing $2 \% \mathrm{X}$-gal and $50 \mu \mathrm{g} / \mathrm{mL}$ ampicillin. Following plating, the agar plates were incubated first at RT for $2 \mathrm{hrs}$, and then at $37{ }^{\circ} \mathrm{C}$ for overnight. Following overnight incubation, distinct white colonies containing the desired aptamer sequences were each aseptically picked and transferred into a glass vial containing $10 \mathrm{~mL}$ LB media and $50 \mu \mathrm{g} / \mathrm{mL}$ ampicillin. Each colony containing solution was incubated overnight at $37^{\circ} \mathrm{C}$ with gentle shaking. 


\subsubsection{Isolation of plasmid DNA}

Plasmid DNA containing the inserted aptamer sequence from the cells was purified using QIAprep Spin Kit following the manufacturer's instructions. Briefly, vials containing $10 \mathrm{~mL}$ overnight cultures were each centrifuged at 3,000 x g for 10 mins before discarding the supernatant from each vial. The pelleted cells were resuspended in $250 \mu \mathrm{L}$ of P1 buffer before transferring the suspension individually from each vial into microcentrifuge tubes. Subsequently, $250 \mu \mathrm{L}$ of P2 buffer was added into each tube and mixed gently by inverting the tubes six times. The resulting suspension in each tube was then incubated at RT for 5 mins to lyse the cells. Thereafter, $350 \mu \mathrm{L}$ of neutralisation buffer (N3) was added into each tube and mixed by inverting the tubes six times before centrifugation at $13,000 \mathrm{x}$ g for 10 mins. The resulting supernatant from each tube was then transferred individually into QIAprep spin columns and centrifuged for $1 \mathrm{~min}$ at $13,000 \times \mathrm{g}$. The flow-through from each column was discarded and the columns were washed using $500 \mu \mathrm{L}$ of PB buffer. Thereafter, $750 \mu \mathrm{L}$ of PE buffer were added to each column to precipitate the plasmid DNA. This was followed by centrifuging the columns at $13,000 \mathrm{x} g$ for $1 \mathrm{~min}$ to remove the PE buffer. After a further centrifugation of the columns at $13,000 \mathrm{x}$ g for $1 \mathrm{~min}$, the collection tube from each column was discarded and the columns were placed individually into microcentrifuge tubes. The plasmid DNA from each column was eluted using $50 \mu \mathrm{L}$ of EB which was added with care directly onto the column filter and incubated at RT for $1 \mathrm{~min}$ before centrifugation at 13,000 x $\mathrm{g}$ for $1 \mathrm{~min}$. Following centrifugation, the columns were discarded and the eluted plasmid DNA from each column was quantified by measuring absorption spectra at $260 \mathrm{~nm}$ using a WPA Biowave II UV-visible Spectrophotometer (Biochrom Ltd, Cambridge, England, UK). The purified plasmid DNA from each column was stored at $-20^{\circ} \mathrm{C}$ until further use. 


\subsubsection{Confirmation of ligation}

Before sequencing, successful insertion of the selected aptamers was confirmed by restriction enzyme digestion of the inserted-plasmid DNA. The digested products were separated by agarose gel electrophoresis, and visualised using the Gel Doc system. Briefly, $10 \mu \mathrm{L}$ of each purified plasmid DNA was added individually into microcentrifuge tubes. This was followed by the addition of $1.5 \mu \mathrm{L}$ of restriction digest buffer (Buffer $\mathrm{H}$ ) and $3.5 \mu \mathrm{L}$ of EcoRI restriction enzyme (containing 10 units) into each tube. Thereafter, all tubes were incubated at $37^{\circ} \mathrm{C}$ for 30 mins. The digestion reaction was stopped by heating each tube to $65^{\circ} \mathrm{C}$ for 5 mins to denature the restriction enzyme. Following denaturation, the digested DNA from each tube was stained using 1.6 $\mu \mathrm{L}$ of 100X SYBR Green dye and subsequently separated by electrophoresis using a $2.5 \%$ agarose gel. The 75 mer DNA bands containing the desired aptamer sequences were confirmed by comparison with a low range DNA ladder when visualised using the Gel Doc system.

\subsubsection{DNA sequencing}

All aptamer samples were sequenced at the DNA sequencing facility of Waikato University (Hamilton, NZ) using an ABI 3130XL instrument fitted with $50 \mathrm{~cm}$ capillary arrays and using Applied Biosystems Big Dye v3.1 dye terminator chemistry.

\subsubsection{Nucleotide analysis}

Nucleotide analysis was undertaken to characterise the isolated aptamers and identify sequence homologies and structural motifs.

\subsubsection{Generation of a phylogenetic tree}

A phylogenetic tree was generated using default Neighbour-Joining option in the Geneious software (http://www.geneious.com) to evaluate the evolutionary lineage within each aptamer population as a result of the SELEX process. 


\subsubsection{DNA alignment}

DNA nucleotide alignment was undertaken using default settings in the Clustal Omega software (https://www.ebi.ac.uk/Tools/msa/clustalo/) to identify key nucleotide regions (homologous sequences) from each aptamer sequence.

\section{$\underline{\text { 2.6.6.3 Two-dimensional (2D) structural analysis }}$}

Web-based mFold, DNA structural folding program, provided by the Zuker laboratory (http://mfold.rna.albany.edu/?q=mfold/DNA-Folding-Form), was used for 2D structural analyses of all identified aptamers. All parameters for the predictions were set as default except for temperature which was set at $23{ }^{\circ} \mathrm{C}$ and ionic condition settings were $150 \mathrm{mM}$ and $2 \mathrm{mM}$ for $\mathrm{Na}^{+}$and $\mathrm{Mg}^{2+}$, respectively.

\subsection{Aptamer characterisation}

Monoclonal TCS aptamers identified from the SELEX procedure were characterised with respect to their [i] target binding affinities and specificities, [ii] $K_{d}$ values, and [iii] insolution conformational change. All monoclonal TCS aptamers utilised in the characterisation studies were commercially synthesised by Life Technologies (Carlsbad, California, USA) on a $1 \mu \mathrm{mol}$ scale with a biotin tag for dot blot assay, or a HEX tag for fluorescent binding assay at the 5'-end of each oligonucleotide sequence, or unlabelled for CD spectroscopy.

\subsubsection{Dot blot assay for binding affinity and specificity}

Binding affinity and specificity of each monoclonal TCS aptamer were characterised using the dot blot assay as described in Section 2.5. The amount of each monoclonal aptamer used in the assay was 50 pmol. 


\subsubsection{Fluorescent binding assay for $K_{d}$ determination}

Monoclonal TCS aptamers with the desired binding properties were subjected to a fluorescent binding assay (Mann et al. 2005) to determine their $K_{d}$ values. In brief, fresh aliquots (each $1 \mathrm{mg}$ ) of TCS D and E matrices were washed three times with $200 \mu \mathrm{L}$ of $1 \mathrm{X}$ BWB and resuspended in $100 \mu \mathrm{L}$ of Blotto solution for 2 hrs at RT. Eight different concentrations, ranging from 80 to $2000 \mathrm{nM}$, of each HEX-labelled aptamer were prepared duplicate in $100 \mu \mathrm{L} 1 \mathrm{X}$ BWB. This was followed by heat-denaturing of each aptamer solution for 10 mins at $90{ }^{\circ} \mathrm{C}$, cooling at $4{ }^{\circ} \mathrm{C}$ for 15 mins and then incubated at RT for 10 mins. Thereafter, the heat-treated aptamer solutions were incubated with aliquots of both TCS D and E matrices for 60 mins at RT with gentle shaking. Unboundaptamers were then discarded by washing with $200 \mu \mathrm{L}$ of $1 \mathrm{X}$ BWB three times. Boundaptamers however were eluted from the aptamer-bound matrices, once with $200 \mu \mathrm{L} 1 \mathrm{X}$ BWB at $95^{\circ} \mathrm{C}$ for 9 mins, and once with $200 \mu \mathrm{LEB}$ at $80^{\circ} \mathrm{C}$ for 9 mins. Both eluates were combined and fluorescent intensity of each eluted aptamer was determined using a PerkinElmer EnSpire Fluorescent reader (PerkinElmer, Waltham, Massachusetts, USA) with excitation and emission wave-lengths of 485 and $535 \mathrm{~nm}$, respectively. As references, the fluorescent binding assay were also performed exactly as described but with the use of control D and E matrices in order to assess non-specific binding of the assayed aptamers to the matrices. The sample volume used in the fluorescent measurement was $180 \mu \mathrm{L}$ per well in a 96-well flat-bottomed polystyrene microtiter plate. Fluorescent readings obtained from each well were used to calculate the amount of eluted aptamers using calibration plots generated from each HEX-labelled aptamer. Based on these data, saturation binding curves for each aptamer being tested were obtained by plotting the amount of eluted aptamer versus the concentration of the incubated aptamer. Assuming an aptamer and its cognate target are binding at 1:1 ratio, the apparent $K_{d}$ value for an aptamer is determined by nonlinear regression for one-site binding according to the equation " $\mathrm{Y}=\mathrm{B}_{\max }{ }^{*} \mathrm{X} /\left(K_{d}+\mathrm{X}\right)$ ", where $\mathrm{Y}$ is degree of saturation, $\mathrm{B}_{\max }$ is the maximum binding sites and $\mathrm{X}$ is the molar concentration of the aptamer. This analysis was performed using Graphpad Prism software (Mehta et al. 2011). 


\subsubsection{Circular dichroism for assessing conformational change}

Monoclonal TCS aptamers with the highest $K_{d}$ values were subjected to CD spectroscopy for assessing the levels of conformational change upon binding to the target. CD measurements of each aptamer were performed using a Chirascan ${ }^{\mathrm{TM}}$ CD Spectrometer (Applied Photophysics, Cambridge, England, UK) at a temperature holding constant at $25^{\circ} \mathrm{C}$ in a quartz cell with an optical path length of $10 \mathrm{~mm}$. The CD spectra were obtained by taking a scan at a scanning rate of $100 \mathrm{~nm} / \mathrm{min}$. Each tested aptamer was suspended in $1 \mathrm{X}$ BWB with $5 \%(\mathrm{v} / \mathrm{v})$ ethanol at $\mathrm{pH} 7.4$ and prepared at $1 \mu \mathrm{M}$ for all CD experiments. TCS was prepared in the same buffer at concentrations of 2,5 , and $10 \mu \mathrm{M}$. In a typical experiment, the CD spectra of each aptamer sample were recorded from 325 to $205 \mathrm{~nm}$ before and after binding to TCS for 60 mins. Buffer baseline spectra were collected in the same cuvette and subtracted from that determined for the sample spectra. Contributions of target spectra were also eliminated by subtraction from the corresponding sample spectra. For comparison, TCS was also incubated with a 75 base-random sequence prepared at $1 \mu \mathrm{M}$ and measured using the same conditions as each tested aptamer. The data were collected in units of millidegrees versus wavelength and were normalised to have zero ellipticity at $325 \mathrm{~nm}$. 


\section{CHAPTER 3}

\section{INITIAL SELEX INVESTIGATION AND THE DEVELOPMENT OF A FLUORESCENT IMAGING ASSAY}

\subsection{Introduction}

This chapter describes experiments using an affinity column-based SELEX procedure for generating ssDNA aptamers to TCS. Specifically, the work presented here outlines the results of experiments using a basic-SELEX procedure which involved the use of a sepharose bead-based partitioning method to capture TCS-specific binding sequences and subsequently, separating them from nonbinding sequences. In addition, the applicability of each method used in the SELEX procedure was evaluated. These investigations included: [i] preparation of target-immobilised affinity matrices; [ii] performing conventional PCR amplifications to enrich selected sequences; [iii] an alkaline denaturation step for preparing ssDNA molecules; [iv] monitoring the SELEX progression using dot blot assay and; [v] characterising selected aptamers using dot blot and fluorescent binding assays. Also investigated was a novel fluorescent imaging method for aptamer characterisation. This imaging method is potentially a more powerful and simpler procedure for assessing binding affinities of newly-generated aptamers. The reasons behind investigating each of the aforementioned methods are explained as followed.

Affinity columns play an important role in generating aptamers binding to small molecules, and there have been recent reports (Kim et al. 2007; Joeng et al. 2009; Jo et al. 2011; Mehta et al. 2012) utilising affinity columns for producing aptamers against targets that are structurally similar to TCS, such as polychlorinated biphenyls (PCBs), BPA, OE2 and diclofenac (DCF) (Figure 3.1). 
Figure 3.1: Compounds with structural similarities to triclosan

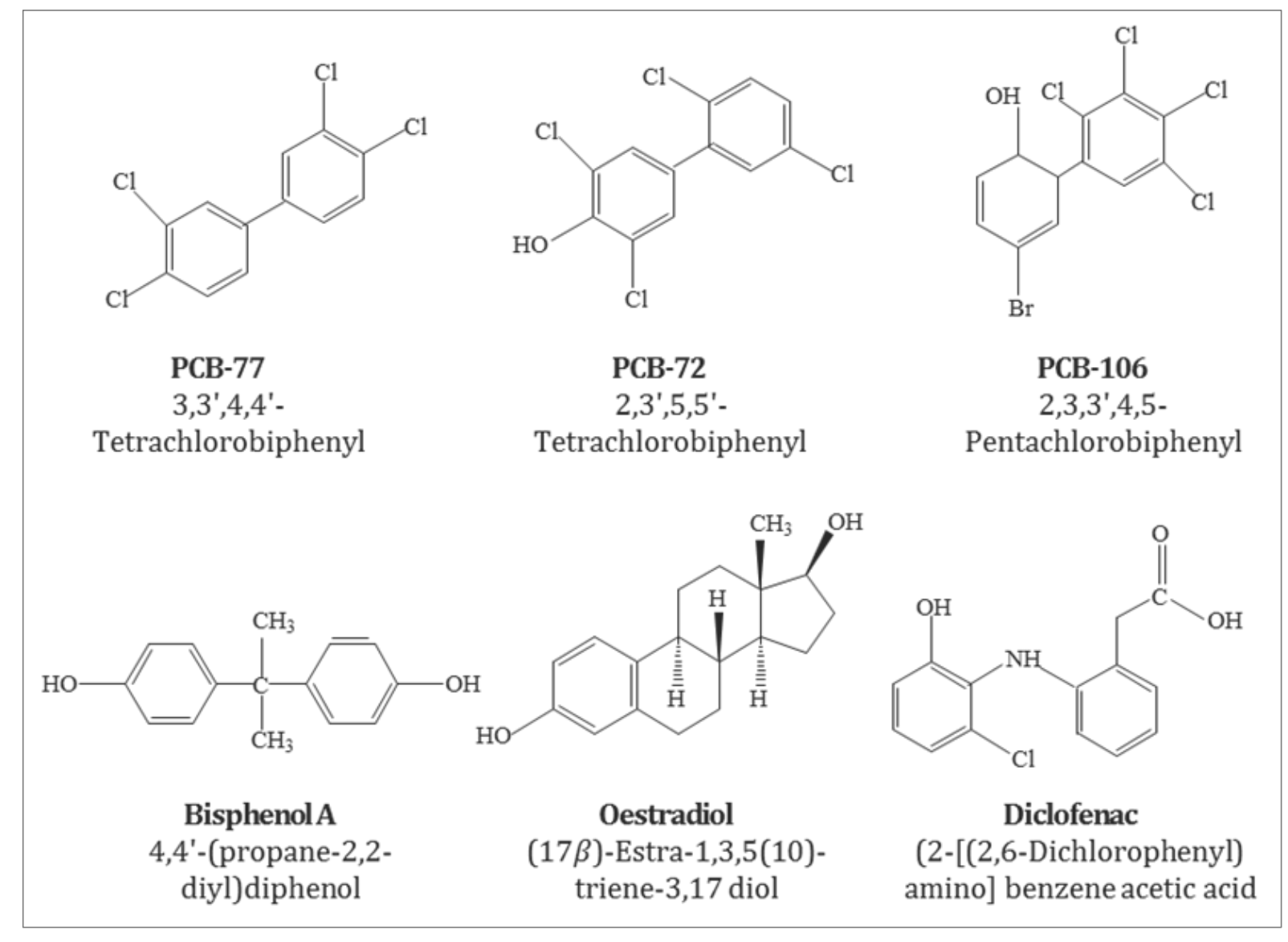

Mehta et al. reported the isolation of an 80 mer ssDNA aptamer capable of binding PCB72 and 106 using a magnetic bead-based SELEX methodology. This aptamer had $K_{d}$ values of 284 and 128 nM for PCB72 and 106 respectively (Mehta et al. 2012). Xu et al. also developed a 76 mer DNA aptamer against PCB77 using an agarose bead-based SELEX methodology, and this aptamer had a micromolar $K_{d}$ value for the target molecule $(\mathrm{Xu}$ et al. 2012). Other examples of using affinity columns for selection include the development of aptamers against DCF (Joeng et al. 2009), BPA (Jo et al. 2011), and OE2 (Kim et al. 2007).

Based on these reports, it was reasoned that a similar strategy might successfully be applied for selecting TCS-specific aptamers. Therefore, TCS was immobilised onto the surface of epoxy-modified sepharose beads using two linker molecules (i.e. DE or ECH) (Figure 3.2). 
Figure 3.2: Conjugation chemistries for the formation of TCS affinity matrices

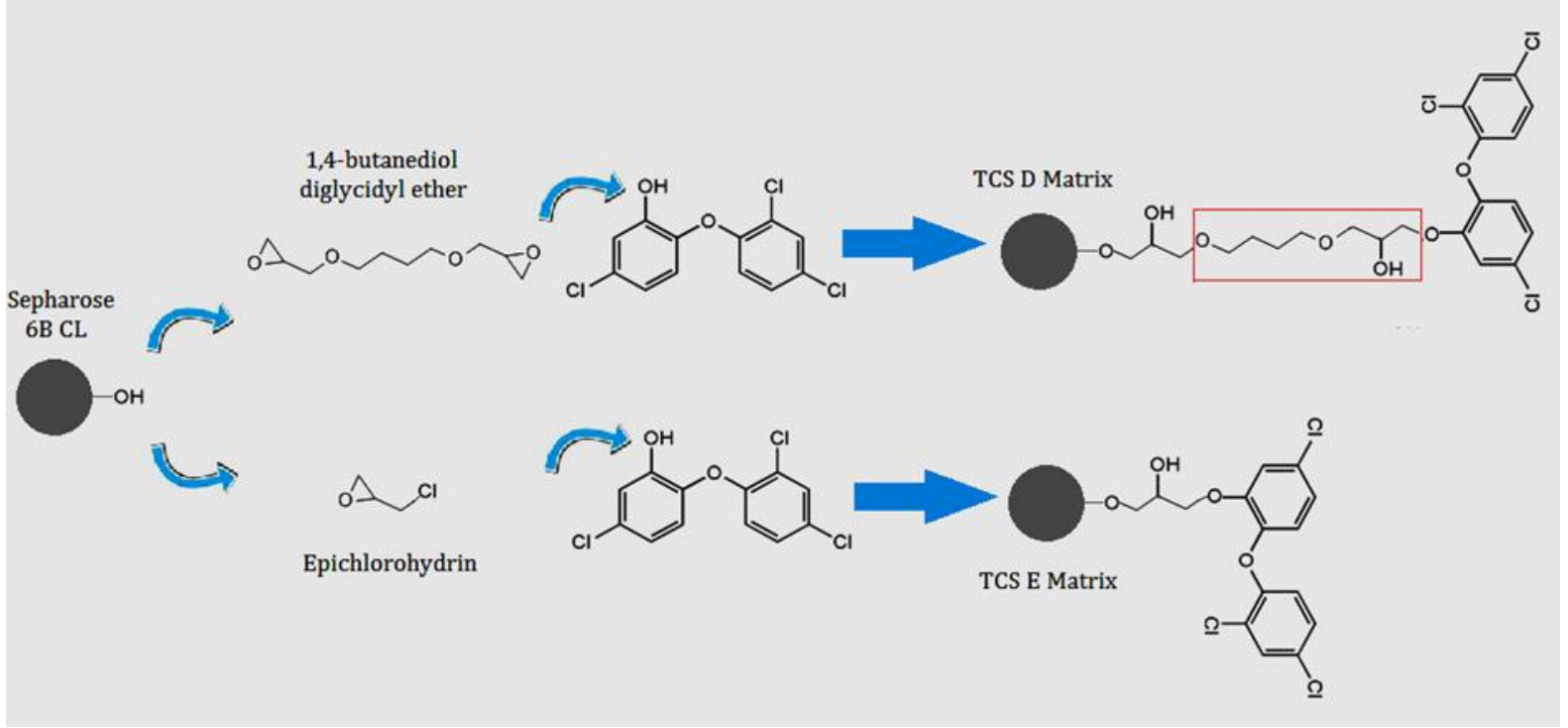

As a result of conjugation, the TCS molecule is nine-atoms further away from the matrix surface in the TCS D matrix (highlighted in Figure 3.2) compared with the TCS E matrix. Both matrices were applied in the basic-SELEX procedure for aptamer selection to investigate whether a longer spacer-arm in the D matrix compared with the E matrix would result in the generation of better quality aptamers (i.e. higher specificity, better binding characteristics, etc). The rationale here was that if the target molecule was kept further away from the surface of the D matrix, then there was likely to be less steric hindrance for recognition by aptamers during selection.

In the-basic SELEX procedure, conventional PCR was chosen due to its availability in the laboratory, and also because the cost for running this form of PCR is lower compared with other PCR techniques such as real-time PCR, emulsion PCR and digital droplet PCR. In addition, the aptamer-bound matrices from each cycle of selection were used as the template for PCR amplification without eluting the bound-aptamers off the matrices. An earlier study by Dr Shalen Kumar reported that the presence of a matrix had no effect on the performance of DNA polymerase used during amplification (Kumar 2014). Another important advantage of not eluting the bound aptamers was that it prevented the loss of highly specific sequences, which might otherwise occur during any elution step. 
As addressed in Chapter 1, alkaline denaturation and fluorescent binding assays have the advantages of convenience and low cost without the need for expensive equipment. Therefore, both methods were chosen for ssDNA preparation and $K_{d}$ determination respectively. The dot blot assay, which was used successfully for characterising aptamers binding to small molecules (i.e. BPA and OE2), was chosen to monitor the progression of the basic-SELEX procedure, and also for aptamer characterisation (Kumar 2014). Finally, experiments utilising a novel fluorescent imaging-based technique were undertaken for aptamer characterisation, but these will be described later.

In summary, the aim of this chapter was to establish a practical laboratory procedure for generating ssDNA aptamers capable of binding to TCS with the objectives of: [i] investigating the utility of a spacer-arm for affinity matrix preparation; [ii] evaluating the dot blot assay for aptamer characterisation and; [iii] determining the $K_{d}$ values for the selected TCS aptamers.

\subsection{Methods}

The methodology described herein refers to that for generating aptamers for TCS using a basic-SELEX procedure including affinity matrix preparation, as well as selection and characterisation of polyclonal and monoclonal aptamers. The methodology also includes the development of a novel fluorescent imaging assay for aptamer characterisation.

\subsubsection{Synthesis of affinity matrices}

Preparation of TCS-conjugated matrices for aptamer selection is described in Chapter 2 (see Section 2.1.1). The resulting TCS D and E matrices, as well as free TCS molecule in water, unmodified sepharose beads and unconjugated matrices (i.e. control D and E matrices) were characterised using the UV-visible spectroscopy (see Section 2.1.1.5, Chapter 2). 


\subsubsection{Basic-SELEX procedure}

The N40 aptamer library and primer sequences used for aptamer selection are listed in Table 2.1 (see Chapter 2). Preparation of all synthesised oligonucleotides and buffers were carried out as outlined in Chapter 2 (see Section 2.3). The various steps of the basicSELEX procedure are described in detail in Chapter 2 (see Section 2.4). The following section provides a summary for selecting TCS-specific aptamers.

\subsubsection{Blocking of affinity matrices}

TCS D and E matrices used for each SELEX cycle were washed and blocked as described in Chapter 2 (see Section 2.4.1).

\subsubsection{Denaturation of ssDNA aptamer library}

For the first round selection, two aliquots (each $3 \mathrm{nmol}$ ) of the synthetic N40 aptamer library were individually suspended in $100 \mu \mathrm{L}$ of $1 \mathrm{X}$ BWB. Thereafter, the resulting library solutions were heat-denatured as described in Chapter 2 (see Section 2.4.2). For subsequent rounds of selection, each aptamer library was subjected to the same treatment.

\subsubsection{Co-incubation of the aptamer library with the affinity matrices}

During the first cycle of SELEX, heat-denatured synthetic library solutions were incubated with Blotto-treated TCS matrices (one aliquot of the N40 library per TCS matrix) overnight at $4{ }^{\circ} \mathrm{C}$ with gentle shaking. For subsequent rounds of selection, the aptamer library generated using TCS D matrix was incubated with the blocked TCS D matrix. Similarly, the library generated using TCS E matrix was incubated with the blocked TCS E matrix.

\subsubsection{Removal of unbound oligonucleotides}

Following incubation, sequences with little or no affinity for TCS were removed by washing each of the aptamer-bound matrices as described in Chapter 2 (see Section 2.4.4). 


\subsubsection{PCR amplification}

For the first round, the entire aptamer-bound TCS D and E matrix preparations were amplified in two parallel PCR reactions (one for each matrix). For subsequent rounds, only half of each matrix preparation was amplified with the remaining half set aside for analysis and in case of PCR failure. The procedures for PCR sample preparation and cycle amplification are outlined in Chapter 2 (see Section 2.4.5). After amplification, the PCR products from each matrix preparation were purified, extracted and strand-separated (see Section 2.4.6-8, Chapter 2) to generate a new aptamer library pool for the next cycle of selection.

\subsubsection{Selection conditions for the basic-SELEX procedure}

The basic-SELEX cycle was repeated eight times to generate TCS-specific aptamers. In order to select high affinity aptamers, the stringency of the SELEX procedure was gradually increased through each cycle by reducing the amount of TCS-conjugated matrices from $50 \mathrm{mg}$ for the first cycle to $10 \mathrm{mg}$ for the last cycle. The decrease in the target molecule is thought to permit greater competition amongst sequences for fewer available targets, and this competition should in theory yield tighter-binding aptamers. Moreover, it is thought that decreasing the incubation time from the initial overnight interval to just $1 \mathrm{hr}$ at the end, would result in newly-generated sequences with the most favourable binding characteristics. Finally, increasing the washing volume after the second cycle of selection by $5 \mathrm{ml}$ per cycle is thought to likely remove weakly-bound sequences from the target molecule. All manipulated selection conditions used in the basic-SELEX procedure are summarised in Table 3.1.

\subsubsection{Progression of the basic-SELEX procedure}

The dot blot assay described in Chapter 2 (see Section 2.5) was utilised to monitor the success of aptamer generation during the basic-SELEX procedure. Polyclonal aptamer libraries (one from each TCS-conjugated matrix) from selection rounds 4, 6 and 8 were subjected to the dot blot assay to assesse their target binding ability. 
Table 3.1: Aptamer selection conditions used in the basic-SELEX procedure

\begin{tabular}{ccccc}
\hline $\begin{array}{c}\text { SELEX } \\
\text { round }\end{array}$ & $\begin{array}{c}\text { Affinity column } \\
\text { (mg) }\end{array}$ & $\begin{array}{c}\text { Incubation } \\
\text { time (hr) }\end{array}$ & $\begin{array}{c}\text { Incubation } \\
\text { temperature }\left({ }^{\circ} \mathbf{C}\right)\end{array}$ & $\begin{array}{c}\text { Wash volume } \\
\text { (mL) }\end{array}$ \\
\hline 1 & 50 & 12 & 4 & 5 \\
2 & 40 & 8 & 4 & 5 \\
3 & 40 & 8 & 4 & 10 \\
4 & 30 & 4 & 4 & 15 \\
6 & 30 & 4 & 4 & 20 \\
6 & 20 & 2 & 4 & 25 \\
7 & 20 & 2 & 4 & 30 \\
8 & 10 & 1 & 4 & 35 \\
\hline
\end{tabular}

\subsubsection{DNA cloning, sequencing and nucleotide analysis}

Following the dot blot assay, and provided the selected aptamers demonstrated successful binding to TCS, the aptamer libraries from round 8 in the basic-SELEX procedure were cloned and sequenced to identify their nucleotide compositions (see Section 2.6.1-5, Chapter 2). A total of twelve plasmid DNA molecules (six from each matrix) were subjected to sequencing, and ten unique sequences (each contained 75 bases) were identified from the sequencing step. These monoclonal sequences were referred to as TCS round 8 (R8) aptamers. Thereafter, nucleotide analyses were performed by: [i] sequence alignment using Clustal Omega software; [ii] generation of a polygenic tree using Geneious software and; [iii] secondary structure prediction using mFold software (see Section 2.6.6, Chapter 2).

\subsubsection{Aptamer characterisation}

Following the evolution of the basic-SELEX process, TCS R8 aptamers were characterised with respect to binding affinity and specificity, as well as the determination of $K_{d}$ values.

\subsubsection{Binding affinity and specificity}

Binding affinity and specificity of TCS R8 aptamers were screened using the dot blot assay described in Section 2.5 (see Chapter 2), except that commercially synthesised R8 aptamers (with a biotin tag at the $5^{\prime}$-end) were utilised in each assay. 
For the binding affinity studies, each biotin-labelled TCS R8 aptamer (50 pmol) was incubated overnight at $4{ }^{\circ} \mathrm{C}$ with nitrocellulose membranes that had been spotted with different amounts of TCS (from 75-600 nmol). For binding specificity studies, TCS R8 aptamers with desired binding properties were also subjected to dot blot assays using compounds sharing structural similarities to TCS. These related molecules were methyltriclosan (MTS), BPA, DCF and OE2. The amount of positive control (i.e. NHS-biotin) varied from 16-2000 pmol.

\subsubsection{Fluorescent binding assay for $K_{d}$ determination}

Following dot blot characterisation, TCS R8 aptamers with the most desirable binding properties were subjected to the fluorescent binding assay to determine their $K_{d}$ values. These experiments were performed exactly as described in Chapter 2 (see Section 2.7.2).

\subsubsection{The development of a novel fluorescent imaging assay}

The development of a fluorescent imaging assay for aptamer characterisation was inspired by Dr Junsheng Lin (Huaqiao University, China) who had selected aptamers against human sperm antigens (personal communication). In Dr Lin's approach, an aptamer specific to sperm surface antigens was labelled with a fluorophore molecule. Upon binding to sperm cells, the resulting aptamer-sperm complexes were then visualised under a fluorescent microscope. Dr Lin found that the labelled-aptamer gathered around each sperm cell and giving rise to fluorescence around the cells. A halo ring around each sperm was observed under the microscope. Similar aptamer-based imaging technique, has been used previously for cancer cell detection and diagnostics (Ma et al. 2015). The present work utilised micrometre-sized sepharose beads, a similar size to sperm cells, for affinity column preparation. It was thought that these beads might be more suitable for developing an imaging-based technique for aptamer characterisation. Preliminary studies established that some of TCS R8 aptamers bound to these beads. Therefore, a simple visualisation of aptamer-bound beads was then used to measure the binding affinity of the selected aptamers. 
The following sections describe a proof of concept experiment for the development of a quantitative procedure, and validation of the fluorescent imaging method for aptamer characterisation.

\subsubsection{Proof of concept}

Aptamer-bound matrices used for imaging experiments were prepared similar to those used in the fluorescent binding assay (see Section 2.7.2, Chapter 2). In brief, a HEXlabelled TCS R8 aptamer (500 nM) was prepared, in duplicate, in $100 \mu \mathrm{L}$ of 1XBWB, and heat-denatured. Thereafter, the aptamer solutions were each added to aliquots (each 1 $\mathrm{mg} / 100 \mu \mathrm{L}$ ) of the blocked control and TCS-conjugated matrices. The resulting aptamermatrix suspensions were incubated at RT for 60 mins. Following incubation, unboundaptamers were discarded by washing the aptamer-bound matrices with $200 \mu \mathrm{L}$ of $1 \mathrm{X}$ BWB three times. The washed aptamer-bound matrices were then resuspended in $30 \mu \mathrm{L}$ 1X BWB. Thereafter, these matrix suspensions were each loaded onto a glass slide with a cover slip on top before being visualised under a Leica DM6000 FS fixed stage fluorescent microscope (Leica Microsystems, Buffalo, Illinois, USA). The microscope was operated first under bright light to find an appropriate region for observation, and then switched to fluorescent mode to acquire fluorescent images. In this initial experiment, the HEX tag was excited under UV, blue and green light sources with the aim of finding the most appropriate light source for excitation. Images were captured under same conditions at each excitation state with $1 \mathrm{sec}$ of exposure time, and the acquired images saved as tagged image file format (TIFF) were then subjected to image analysis.

\subsubsection{Image analysis}

The captured images were subjected to image analyses to provide quantitative information regarding binding affinity of each aptamer to the control and TCS-conjugated matrices. The image analysis process was performed using Igor Pro 6 (Wavemetrics, https://www.wavemetrics.com/index.html), a scientific graphic and image processing software used widely by researchers (Gomez et al. 2002). 
In detail, each captured image was loaded into the software as a TIFF file. Then the image was divided into three layers corresponding to red, green and blue colour code. The layer with highest fluorescent intensity was chosen for the analytical process. In the chosen layer, fluorescent beads were presented as white spots, whereas surrounding buffer region remained black. Thereafter, regions of interest (ROI) on the beads were chosen randomly as circles with a sum of $100 \pm 3 \mathrm{~cm}$ in diameter (which remained constant for each analysed image) and light intensity within ROI was automatically averaged by the

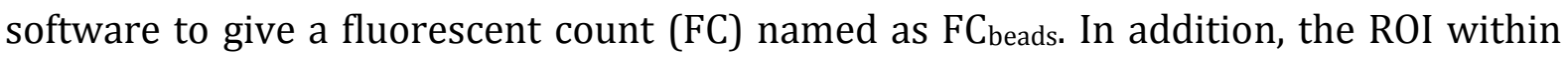
surrounding buffer was also chosen as random shapes and light intensities were also averaged to give a FC named as FC buffer. The mean FC (FCmean) defined as the difference between the ROI of beads and buffer, was then calculated by subtracting $\mathrm{FC}_{\text {buffer }}$ from $\mathrm{FC}_{\text {beads. }}$ The $\mathrm{FC}_{\text {mean }}$ representing the strength of fluorescent intensity within each image was used to indicate the affinity strength of an aptamer to its cognate target.

\section{$\underline{3.2 .6 .3 \text { Validation }}$}

The applicability of the fluorescent imaging method for aptamer characterisation was validated by applying the method to assess the binding affinities of TCS R8 aptamers to both the control and TCS-conjugated matrices. The $K_{d}$ value of a chosen aptamer was also determined using the imaging method. In both studies, preparation of aptamer-bound matrices was carried out as described Section 3.2.6.1, except that each aptamer being tested in the binding affinity study was prepared at one concentration of $500 \mathrm{nM}$, whereas the aptamer used in the $K_{d}$ study was prepared at eight different concentrations ranging from 80-2000 nM. Fluorescent images captured from both studies were analysed using the image analysis methodology (see Section 3.2.6.2). Binding affinty of each aptamer in the affinity study was ranked based on the values of $\mathrm{FC}_{\text {mean. }}$. Binding saturation curves for the $K_{d}$ study were plotted using $\mathrm{FC}_{\text {mean }}$ versus aptamer concentration, and the $K_{d}$ value was then determined using GraphPad Prism software (see Section 2.7.2, Chapter 2). 


\subsection{Results}

\subsubsection{Preparation of affinity matrices}

Successful conjugation of TCS to the sepharose beads was confirmed by obtaining the UVvisible absorption spectra of TCS D and E matrices (Figure 3.3).

Figure 3.3: UV-visible spectra of (A) TCS in water; (B) unmodified sepharose beads (blue), control D matrix (red), and control E matrix (green); (C) TCS D matrix; and (D) TCS E matrix
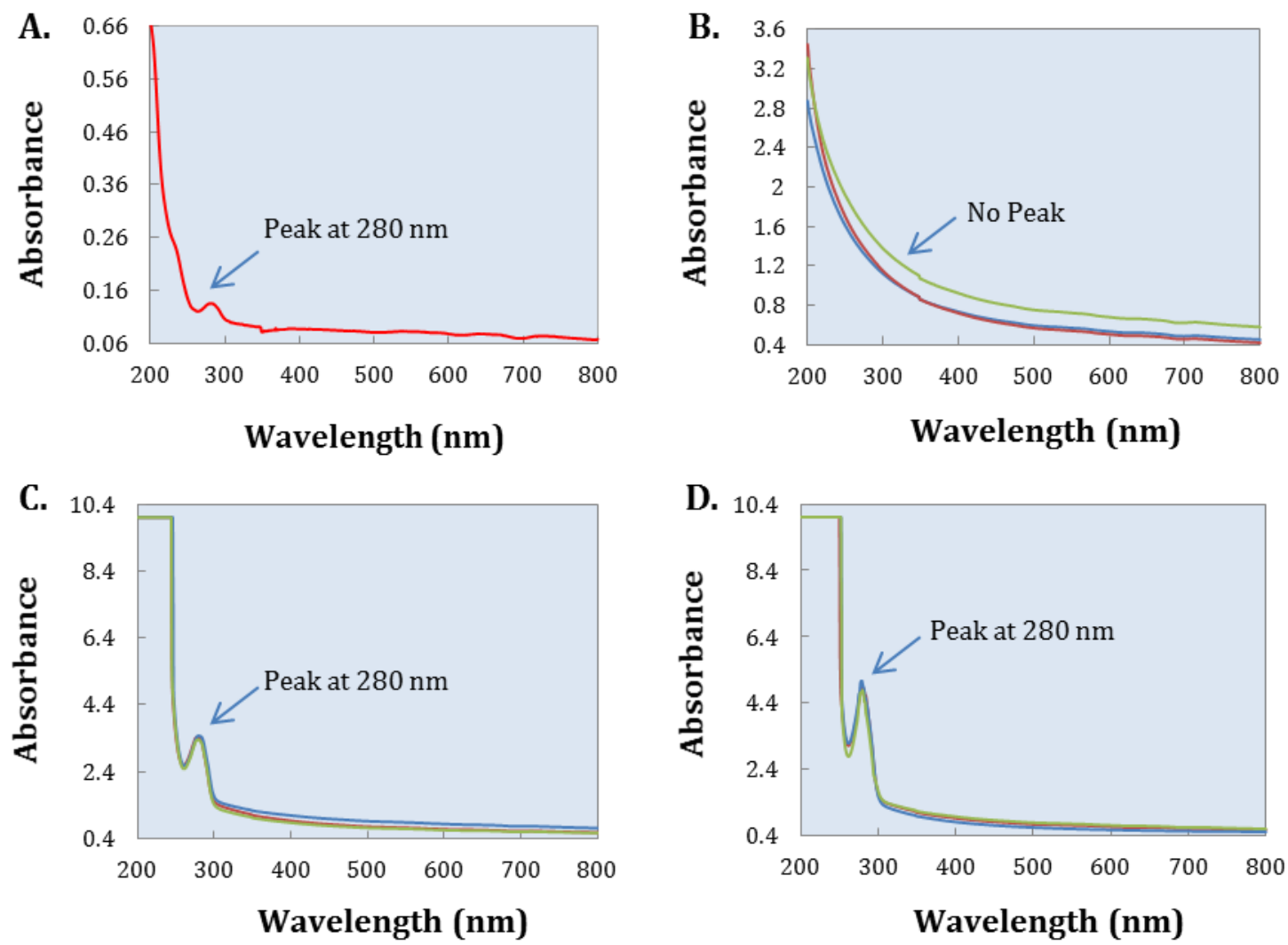

The key characteristic difference between TCS-conjugated (Figure 3.3 C and D) and unconjugated (Figure $3.3 \mathrm{~B}$ ) matrices was the presence of an absorption peak at $280 \mathrm{~nm}$ in the conjugated matrices. The absorption peak was also observed in free TCS prepared in water (Figure $3.3 \mathrm{~A}$ ), denoting the presence of TCS molecules on the surface of the conjugated matrices. 


\subsubsection{PCR amplification}

Conventional PCR was carried out throughout the basic-SELEX procedure to enrich the selected aptamer libraries. One example of the PCR results is shown in Figure 3.4.

Figure 3.4: PCR amplification of the basic-SELEX round 8 aptamer-bound matrices for dot blot assays

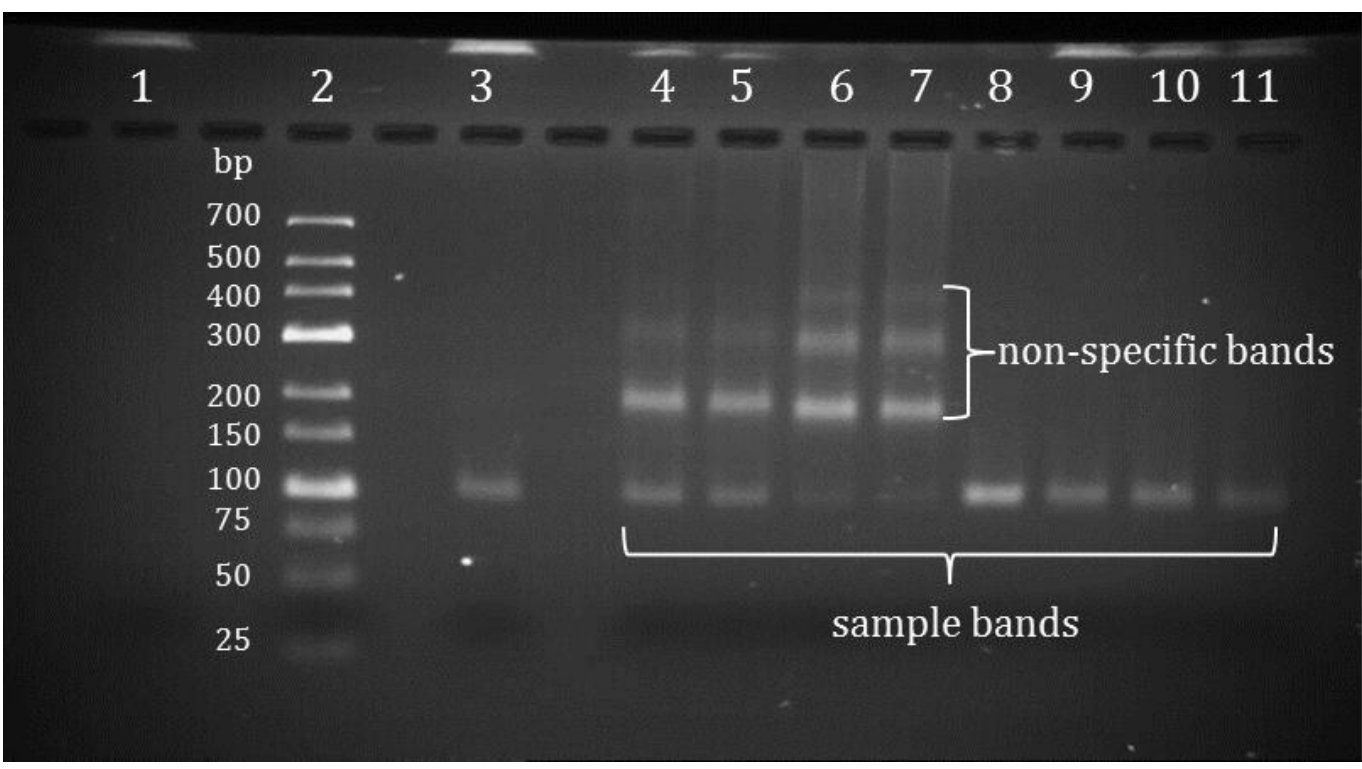

Lane 1: -ve control (no template); lane 2: DNA ladder; lane 3: +ve control (1 nM N40 library); lanes 4-7: sample bands amplified from TCS D matrix; lanes 8-11: sample bands amplified from TCS E matrix and; bp = base pair.

Non-specific PCR by-products were formed during the amplification of aptamer-bound TCS D matrix (Figure 3.4, lanes 4-7). Only sample bands (75-bp) were excised for dot blot assay. When amplifying the R8 aptamer-bound TCS D and E matrices for selection, nonspecific bands were also observed (data not shown), resulting in a significant loss in the recovery of 75 mer aptamers for the subsequent round of selection.

\subsubsection{Progression of the basic-SELEX procedure}

Polyclonal aptamer libraries generated from selection rounds 4, 6 and 8 were subjected to dot blot assays to evaluate their binding affinity to TCS (Figure 3.5). 
Figure 3.5: Dot blot assays for polyclonal TCS aptamers from the basic-SELEX rounds 4, 6, and 8 selected using (i) long-arm TCS D matrix; (ii) short-arm TCS E matrix and; (iii) control experiments

\section{(i) Polyclonal TCS D aptamers}

\begin{tabular}{|c|r|r|r|r|r|}
\hline \multirow{2}{*}{$\begin{array}{c}\text { TCS D } \\
\text { aptamers }\end{array}$} & \multicolumn{5}{|c|}{ TCS dot blots (nmol) } \\
\cline { 2 - 6 } & 600 & 300 & 150 & 75 & 0 \\
\hline Round 4 & & & & & \\
\hline Round 6 & & & & & \\
\hline Round 8 & & & & & \\
\hline
\end{tabular}

\section{(ii) Polyclonal TCS E aptamers}

\begin{tabular}{|c|r|r|r|r|r|}
\hline \multirow{2}{*}{$\begin{array}{c}\text { TCS E } \\
\text { aptamers }\end{array}$} & \multicolumn{5}{|c|}{ TCS dot blots (nmol) } \\
\cline { 2 - 6 } & 600 & 300 & 150 & 75 & 0 \\
\hline Round 4 & & & & & \\
\hline Round 6 & & & & & \\
\hline Round 8 & & 0 & & & \\
\hline
\end{tabular}

\section{(iii) Positive and negative controls}

\begin{tabular}{|c|c|c|c|c|c|c|}
\hline \multirow{2}{*}{$\begin{array}{r}(+v e) \\
\text { Control }\end{array}$} & \multicolumn{5}{|c|}{ NHS-biotin dot blots (nmol) } & \multirow{2}{*}{$\begin{array}{c}\text { (-ve) } \\
\text { control }\end{array}$} \\
\hline & 10 & 1 & 0.1 & 0.01 & 0 & \\
\hline Round 4 & & & & & & \\
\hline Round 6 & & & 0 & & & \\
\hline Round 8 & & & & & & \\
\hline TCS & - & - & - & - & - & + \\
\hline Aptamer & - & - & - & - & - & - \\
\hline ST-HRP & + & + & + & + & + & + \\
\hline
\end{tabular}

TCS = triclosan; ST-HRP = streptavidin-horseradish peroxidase; NHS = N-hydroxysuccinimide; "+" means the presence of and; "-" means not present. 
As shown in Figure $3.5 \mathrm{i}$ and ii, there were no positive dot blot signals after 4 rounds of selection using either TCS D or E matrix. However, visible dots began to develop at round 6, and became intense at round 8 . These polyclonal R8 aptamers from both matrices showed strong binding to TCS at 600 and $300 \mathrm{nmol}$. Control samples showed that the dot blot experiments had similar sensitivities with a $L O D$ down to $0.1 \mathrm{nmol}$, and there were no non-specific bindings by the enzyme ST-HRP to TCS without aptamers (Figure 3.5 iii).

\subsubsection{DNA cloning, sequencing and nucleotide analysis}

Twelve plasmid DNA (each containing one monoclonal aptamer insertion) from the basicSELEX procedure were sequenced. Clones D1 to D6 were produced using TCS D matrix, whilst E1 to E6 were the products of TCS E matrix. Two clones, D3 and E4, were more than 75 bases in length, and were therefore excluded from further studies (data not shown). The remaining clones (each 75 bases) are shown in Table 3.2.

Table 3.2: Nucleotide sequences of basic-SELEX round 8 TCS aptamers (Clones D and E) and the composition (\%) of individual bases (A, T, G, and $\mathrm{C}$ ) in the $\mathrm{N} 40$ random region within each aptamer

\begin{tabular}{clcccc}
\hline Clone & \multicolumn{1}{c}{ Full sequence 5' to 3' $^{\prime}$} & A & T & G & C \\
\hline D1 & $\begin{array}{l}\text { ATACGAGCTTGTTCAATAGGATCAATTTCTGAAGCCTG } \\
\text { TTATTGTAAGGTCGTTCGCATGATAGTAAGAGCAATC }\end{array}$ & 22.5 & 35 & 25 & 17.5 \\
\hline $\mathbf{D 2}$ & $\begin{array}{l}\text { ATACGAGCTTGTTCAATAGTGAATTTTGTGTACGCACA } \\
\text { CAGCTGTGCTCGATGTCTGGTGATAGTAAGAGCAATC }\end{array}$ & 17.5 & 32.5 & 30 & 20 \\
\hline $\mathbf{D 4}$ & $\begin{array}{l}\text { ATACGAGCTTGTTCAATATGTAGTGATTAAATGTCCAG } \\
\text { TTGGGTCCAATAGCATTCCGTGATAGTAAGAGCAATC }\end{array}$ & 25 & 32.5 & 25 & 17.5 \\
\hline $\mathbf{D 5}$ & $\begin{array}{l}\text { ATACGAGCTTGTTCAATATTAATGCTTCTGCGTAGAAG } \\
\text { TGCGGTAGGATGCGTATGGGTGATAGTAAGAGCAATC }\end{array}$ & 20 & 30 & 37.5 & 12.5 \\
\hline $\mathbf{D 6}$ & $\begin{array}{l}\text { ATACGAGCTTGTTCAATAGCAGTGACTTTCCTGGTATC } \\
\text { TTGTTAACAGGTTGTCTGTATGATAGTAAGAGCAATC }\end{array}$ & 17.5 & 40 & 25 & 17.5 \\
\hline $\mathbf{E 1}$ & $\begin{array}{l}\text { ATACGAGCTTGTTCAATACTGTGTGTTGTTATCGTTCA } \\
\text { GCAAGTGGAATGGGTATGTGTGATAGTAAGAGCAATC }\end{array}$ & 17.5 & 37.5 & 35 & 10 \\
\hline $\mathbf{E 2}$ & $\begin{array}{l}\text { ATACGAGCTTGTTCAATACGCGATCGTTCGATGCGTTA } \\
\text { TGTATTTGTGTGGCGCCGTGTGATAGTAAGAGCAATC }\end{array}$ & 10 & 35 & 35 & 20 \\
\hline $\mathbf{E 3}$ & $\begin{array}{l}\text { ATACGAGCTTGTTCAATACTTGCGTTAGTTTTGCTTGG } \\
\text { GACGGGGTGCTTGTTGTTGATGATAGTAAGAGCAATC }\end{array}$ & 7.5 & 42.5 & 37.5 & 12.5 \\
\hline $\mathbf{E 5}$ & $\begin{array}{l}\text { ATACGAGCTTGTTCAATATTGGAACCGAAATGTTGATG } \\
\text { TGTGGGTGTTTCGTTTGGAATGATAGTAAGAGCAATC }\end{array}$ & 20 & 37.5 & 35 & 7.5 \\
\hline $\mathbf{E 6}$ & $\begin{array}{l}\text { ATACGAGCTTGTTCAATACATGTTTCATGTTGGTTATC } \\
\text { TTTTGGACTTCAATGCGGTGTGATAGTAAGAGCAATC }\end{array}$ & 15 & 45 & 25 & 15 \\
\hline
\end{tabular}

Highlighted nucleotides represent forward (red) and reverse (blue) primer regions. 
Analysis of the N40 random region for each TCS R8 aptamer indicated an enrichment of $\mathrm{T}$ and $\mathrm{G}$ nucleotides with $\mathrm{T}$ being the most abundant occupying over $30 \%$ of the $\mathrm{N} 40$ region (Table 3.2).

Phylogenetic analysis of TCS R8 aptamers was undertaken to elucidate their evolutionary relationships (Figure 3.6).

Figure 3.6: Phylogenetic relationships among TCS round 8 aptamers D (red) and E (blue) clones

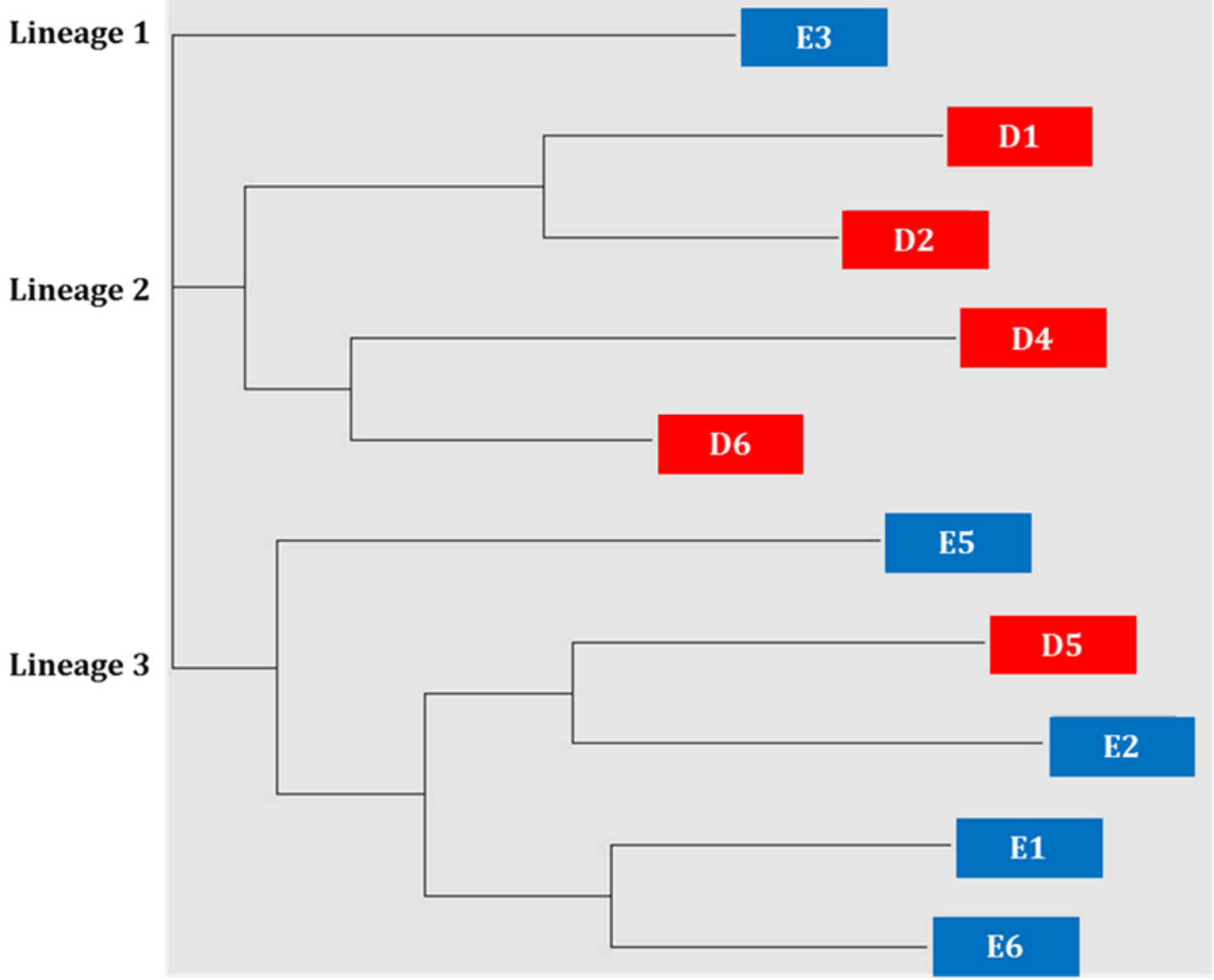

The phylogenetic tree (Figure 3.6) indicated one distinct aptamer E3 alone in lineage 1, whereas the others were incorporated into two major branches of evolutionary lineage (i.e. 2 \& 3). Aptamers D1, D2, D4 and D6 were grouped together in lineage 2: these were generated using TCS D matrix for selection. Surprisingly, aptamer D5, which was selected using the TCS D matrix, showed more evolutionary similarity with aptamers from the TCS E matrix, and grouped with E1, E2, E5 and E6 in lineage 3. 
Nucleotide alignment in the N40 region for TCS R8 aptamers and positions with conserved sequences were then analysed (Figure 3.7).

Figure 3.7: Alignment of the N40 regions for TCS round 8 aptamers (D and E clones)

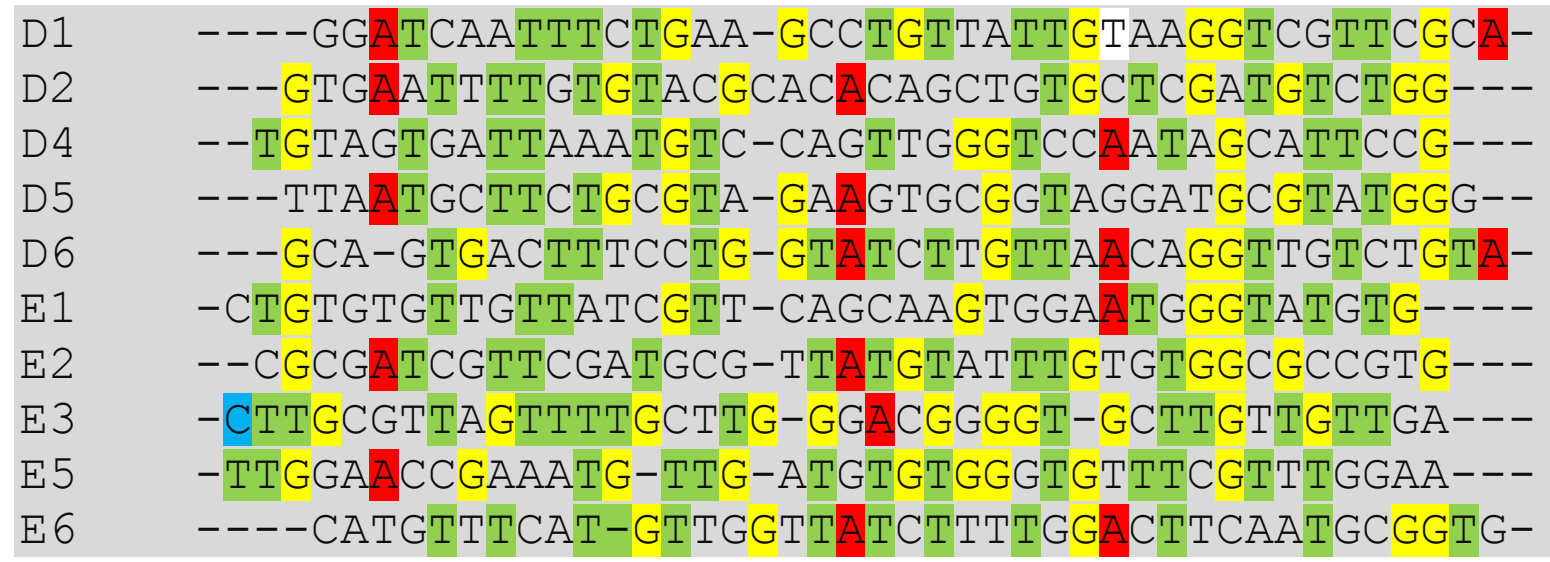

Highlighted sequences denoted the regions of homology.

Analysis of the N40 regions revealed no conserved regions among the R8 TCS aptamers, but duplicate, triplicate and even quadruplet copies of $\mathrm{T}$ and $\mathrm{G}$ nucleotides, as well as duplicates and triplicates of A nucleotides were common features (Figure 3.7).

Secondary 2D structures of TCS R8 aptamers were predicted in order to find their folding characteristics between the primer and random regions. The secondary structures of aptamers D5 and E6 are shown in Figure 3.8, and the complete folding are shown in Appendix II. 
Figure 3.8: Secondary structures of aptamers D5 (TR8D5) and E6 (TR8E6) demonstrated a three stem-loop (SL) structure

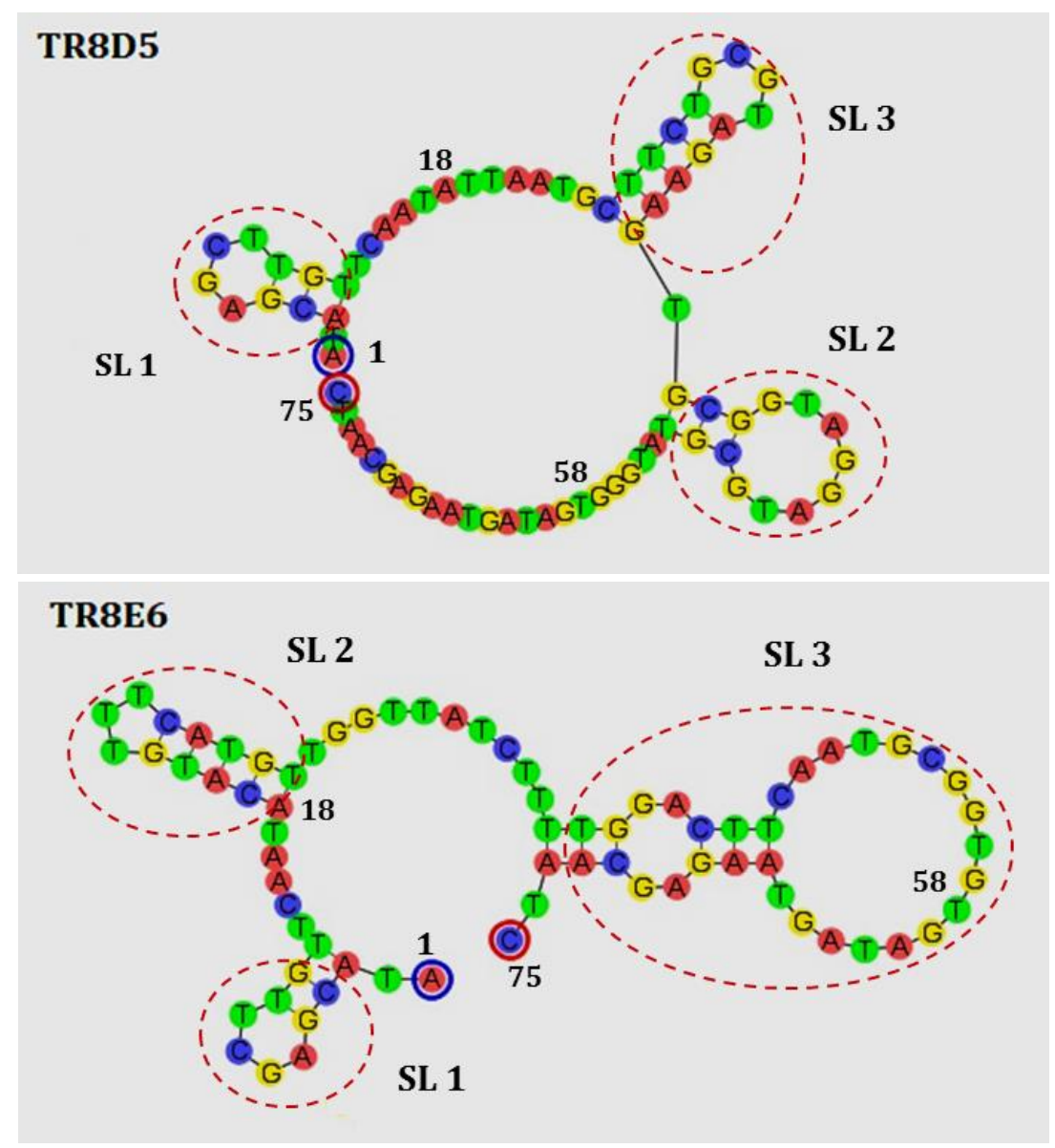

According to the folded-secondary structures (Figure 3.8 and Appendix II), three aptamers (i.e. D1, E2, and E3) were folded into similar linear stem-loop structures. Two aptamers (i.e. D6 and E5) showed two stem-loop structures, four aptamers (i.e. D2, D5, E1 and E6) showed three stem-loop structures, and aptamer D4 showed a four stem-loop structure.

\subsubsection{Aptamer characterisation}

TCS R8 aptamers (i.e. D1, D2, D4, D5, D6, E1, E2, E3, E5 and E6) were characterised with respect to their binding intensity and sensitivity (i.e. detection limits), and aptamers (i.e. D4, D5, D6, E5 and E6) were also tested for their binding specificity to TCS using the dot blot assay. Subsequently, the $K_{d}$ values of two aptamers D5 and E6 were determined using the fluorescent binding assay. 


\subsubsection{Binding intensity and sensitivity}

The binding of each TCS R8 aptamer was tested over different amounts of TCS (75 to 600 nmol). The results from dot blot assays are summarised in Figure 3.9, and the results for control experiments are shown in Appendix III.

Figure 3.9: Binding intensity and sensitivity of TCS round 8 aptamers (D and E clones)

\begin{tabular}{|c|c|c|c|c|c|}
\hline \multirow{2}{*}{$\begin{array}{l}\text { Aptamer } \\
\text { clone }\end{array}$} & \multicolumn{5}{|c|}{ TCS dot blots (nmol) } \\
\hline & 600 & 300 & 150 & 75 & 0 \\
\hline D1 & & & & & \\
\hline D2 & & & & & \\
\hline D4 & & & & & \\
\hline D5 & & & & & \\
\hline D6 & C & & & & \\
\hline E1 & & & & & \\
\hline E2 & & & & & \\
\hline E3 & 3 & & & & \\
\hline E5 & & & & & \\
\hline E6 & $\smile$ & 6 & & & \\
\hline
\end{tabular}


TCS R8 aptamers were classified into three categories based on the intensity and sensitivity of dot blot signals (Figure 3.9). The best candidates were aptamers D4, D5, D6, E5 and E6 as these showed strong positive binding at 600 and $300 \mathrm{nmol}$ of TCS. The next best candidates were aptamers D2 and E3 which also showed positive binding down to $300 \mathrm{nmol}$, but with signal intensity much weaker compared with the aforementioned aptamers. Finally, aptamers D1, E1 and E2 were considered to be the poorest of those selected because of their weak binding signals with only light spots at $600 \mathrm{nmol}$. Furthermore, none of the aptamers showed binding to TCS at 150 and $75 \mathrm{nmol}$.

\subsubsection{Binding specificity}

To verify binding specificity, the best TCS R8 aptamers (i.e. D4, D5, D6, E5 and E6 ) were each subjected to additional dot blot assays (Figure 3.10) using structurally similar compounds (i.e. MTS, BPA, DCF and OE2).

Figure 3.10: Dot blot assay for screening the binding specificities of TCS round 8 aptamers (i) D4, (ii) D5, (iii) D6, (iv) E5 and (v) E6 to TCS (triclosan), MTS (methyltriclosan), BPA (bisphenol A), DCF (diclofenac) and OE2 (oestradiol).

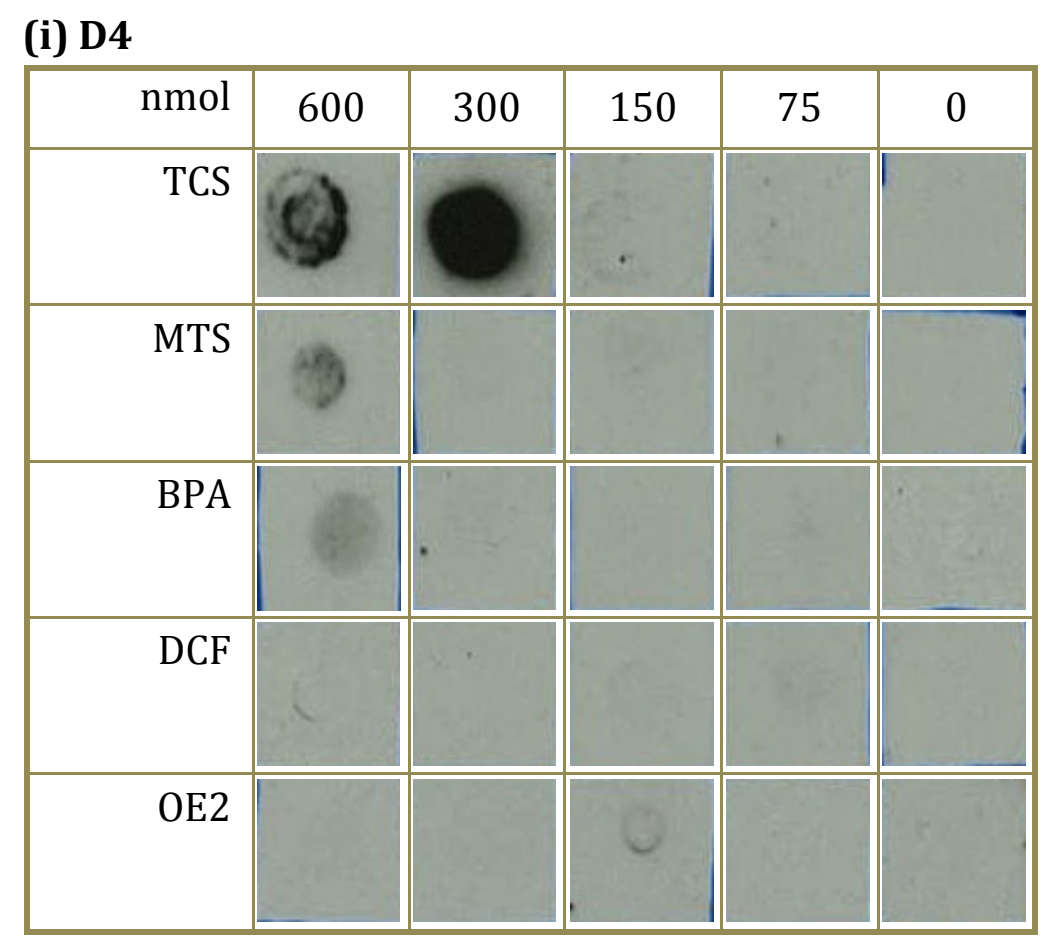

Aptamer D4 showed positive binding to TCS at 600 and 300 nmole. Non-specific interaction was observed with MTS and BPA at 600 nmoles, with very faint signals observed with DCF at $600 \mathrm{nmol}$ and OE2 at $150 \mathrm{nmole}$. 
(ii) D5

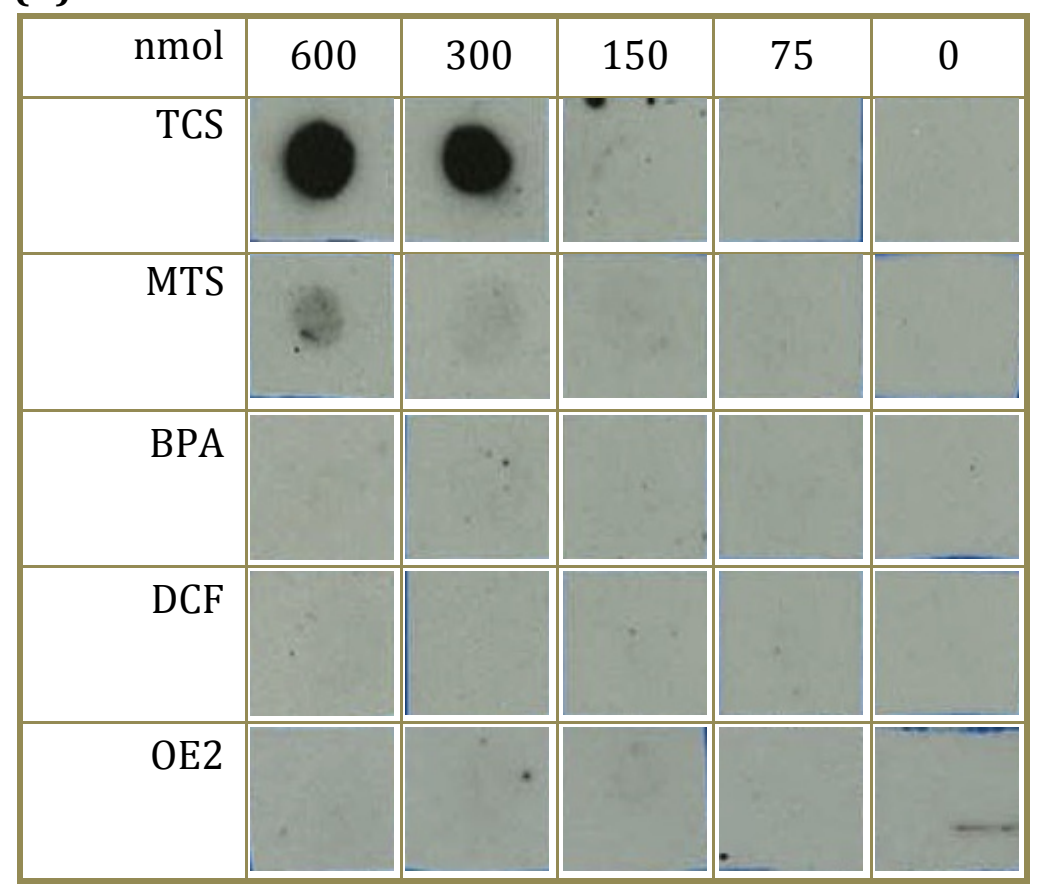

Aptamer D5 showed positive binding to TCS at 600 and 300 nmol. Non-specific interaction was observed with MTS at 600,300 , and $150 \mathrm{nmol}$. No visible signals were observed with BPA, DCF and OE2. (iii) D6

\begin{tabular}{|r|r|r|r|r|r|}
\hline nmol & 600 & 300 & 150 & 75 & 0 \\
\hline TCS & \multirow{2}{*}{6} & & & & \\
\hline MTS & & & & & \\
\hline BPA & & & & & \\
\hline DCF & & & & & \\
\hline & & & & & \\
\hline OE2 & $*$ & & & & \\
\hline & & & & & \\
\hline
\end{tabular}

Aptamer D6 showed positive binding at 600 and $300 \mathrm{nmol}$ to TCS, MTS, BPA, DCF and OE2. In addition, visible signals were observed with $150 \mathrm{nmol}$ of TCS, MTS and BPA. Faint signals were also observed at 75 nmoles of MTS and BPA. 


\section{(iv) E5}

\begin{tabular}{|c|c|c|c|c|c|}
\hline nmol & 600 & 300 & 150 & 75 & 0 \\
\hline TCS & & & & & \\
\hline MTS & & & & & \\
\hline BPA & & & & & \\
\hline DCF & & & & & \\
\hline OE2 & & & & & \\
\hline & & & & & \\
\hline
\end{tabular}

Aptamer E5 showed positive binding to TCS at 600 and 300 nmol. Non-specific interaction was observed with MTS at $600 \mathrm{nmol}$ and BPA at 600, 300, and $150 \mathrm{nmol}$.

\section{(v) E6}

\begin{tabular}{|r|r|r|r|r|r|}
\hline nmol & 600 & 300 & 150 & 75 & 0 \\
\hline TCS & & & & & \\
\hline MTS & & & & & \\
\hline BPA & $\cdot$ & & & & \\
\hline DCF & & & & & \\
\hline OE2 & & & & & \\
\hline & & & & & \\
\hline
\end{tabular}

Aptamer E6 showed positive binding to TCS at 600 and 300 nmol. Non-specific interaction was observed with MTS at 600 and $300 \mathrm{nmol}$. 
Based on results from Figure 3.10, aptamer D6 showed binding to all reagents tested (Figure $3.10 \mathrm{iii}$ ), and was therefore excluded for further characterisation. For the remaining aptamers tested, weak dot blot signals were observed with DCF and OE2, but strong binding was noted for MTS (Figure $3.10 \mathrm{i}$, ii, iv, and iv). In addition, aptamers D4 and E5 also showed affinity to BPA (Figure $3.10 \mathrm{i}$ and iv) with E5 being able to detect BPA at concentrations as low as $150 \mathrm{nmol}$. Only aptamers D5 and E6 showed the least amount of binding to the structurally similar compounds, and both aptamers also demonstrated strong affinities to TCS (Figure $3.10 \mathrm{ii}$ and v). Therefore, aptamers D5 and E6 were chosen for further characterisation to determine their $K_{d}$ values.

\subsubsection{Fluorescent binding assay for $K_{d}$ determination}

The $K_{d}$ values for the chosen aptamers D5 and E6 were determined using the fluorescent binding assay. The concentrations of each aptamer being tested varied from 40 to 1000 $\mathrm{nM}$, whilst the amount of TCS-conjugated matrices used in each assay was kept constant. Control matrices (D \& E) were also assayed as references to assess the extent of nonspecific binding by both aptamers. Saturation binding curves were plotted by measuring the amount of matrix-bound aptamer versus the corresponding input-aptamer concentration used in the assay (Figure 3.11). 
Figure 3.11: Saturation curves of (A) aptamer D5 to control and TCS D matrices; (B) aptamer E6 to control and TCS E matrices and; normalised saturation curves of (C) aptamer D5 and (D) aptamer E6 after subtracting the non-specific binding to control matrix from the specific binding to TCS-conjugated matrix

A.

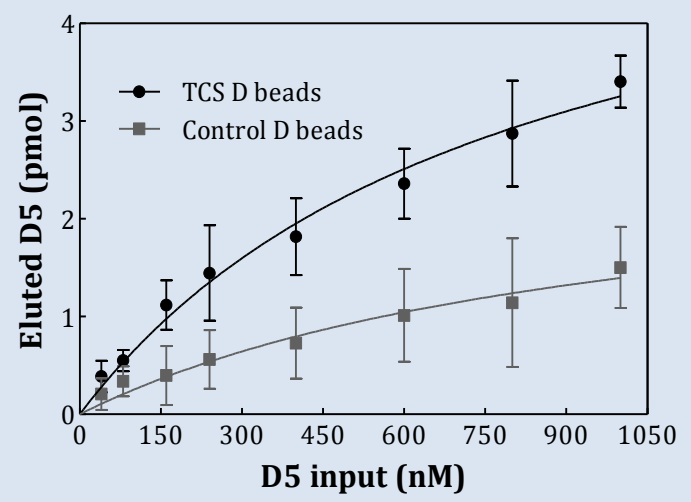

C.

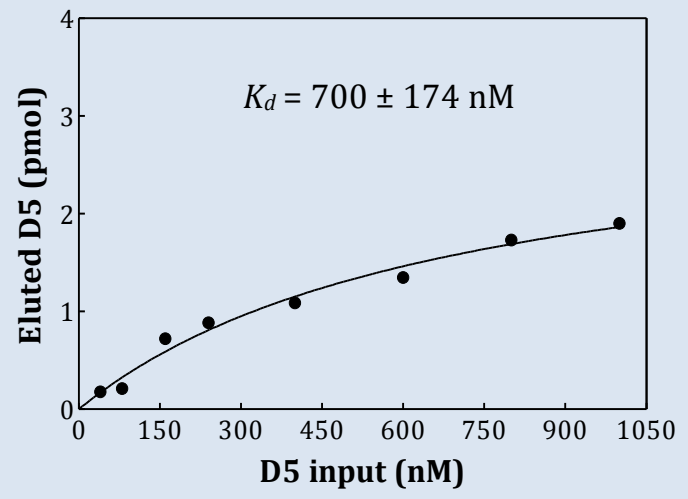

B.

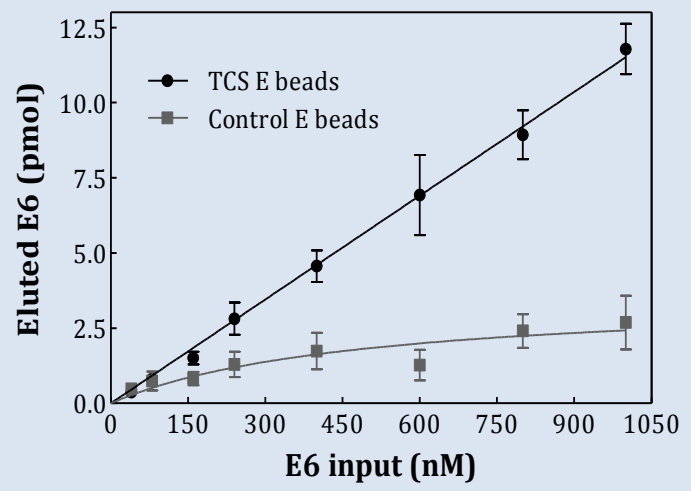

D.

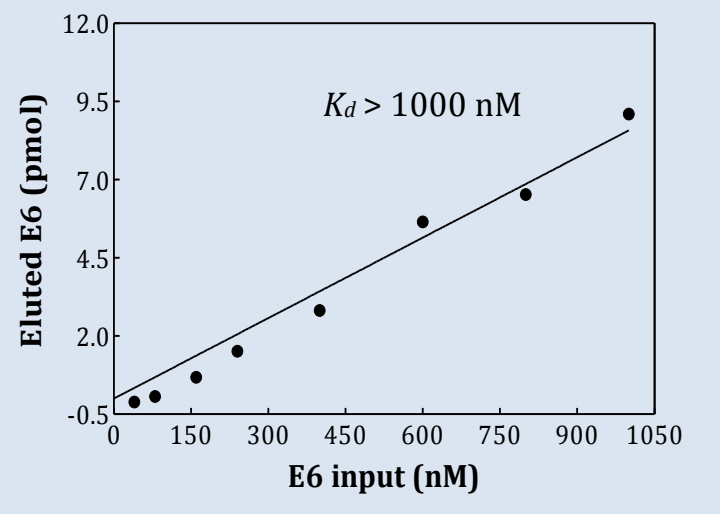

The error bars in A and B represent the standard deviation of the amount of eluted aptamers measured via four independent experiments.

Under the assay conditions, aptamer D5 showed a higher affinity to TCS compared with aptamer E6, as the binding curves of D5 approached saturation, whereas E6 showed a linear increase with no signs of saturation up to $1000 \mathrm{nM}$ aptamer (Figure 3.11 A and B). The mean \pm SD $K_{d}$ value for aptamer D5 was determined to be $700 \pm 174 \mathrm{nM}$, whereas the $K_{d}$ value for E6 was estimated to be more than $1 \mu \mathrm{M}$ (Figure 3.11 C and D). 


\subsubsection{The development of a fluorescent imaging assay}

The applicability of a fluorescent imaging method for aptamer characterisation was investigated after first confirming that the TCS-conjugated matrix elicited fluorescent signals upon binding with a HEX-labelled TCS aptamer. The HEX-labelled aptamer E6 (HEX-E6) was used for binding studies with both control and TCS E matrices. The resulting aptamer-bound matrices were visualised under differed light sources (i.e. UV, blue and green). Fluorescent images were taken under each light excitation, and the captured images are summarised in Figure 3.12a.

Figure 3.12a: Fluorescent microscope operation for capturing fluorescent images from HEX-labelled aptamer E6 (HEX-E6) binding to control and TCS E matrices

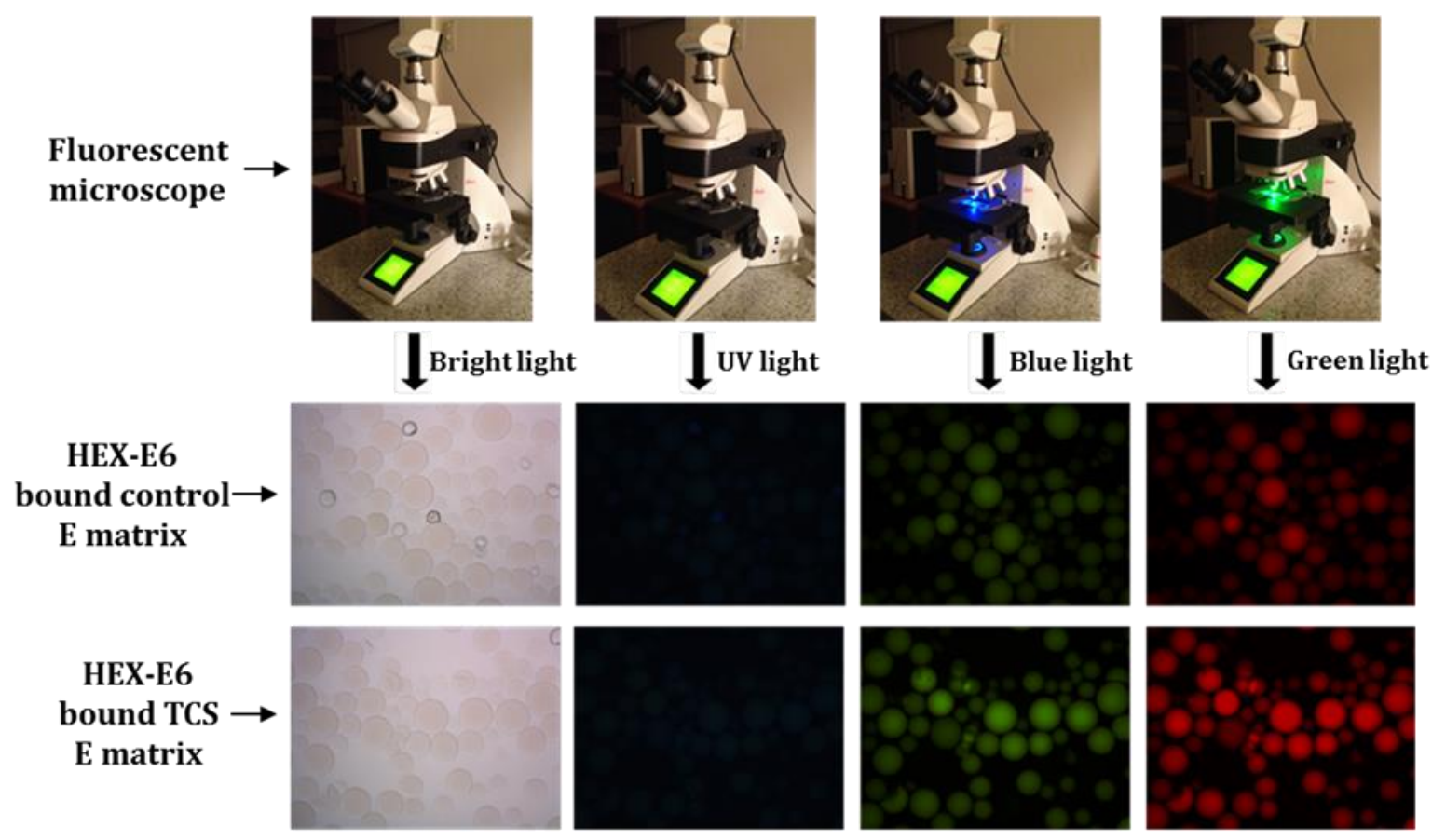

Fluorescent images were captured under different light sources with 1 second of exposure time and; UV = ultraviolet.

From the images in Figure 3.12a, it is evident that upon binding with the HEX-E6, both control and TCS E matrix were capable of fluorescence under either blue or green light excitation. In addition, it was observed that fluorescent signals emitted from the TCS E matrix were stronger when compared with the control E matrix. Furthermore, the green light was consistently better than blue light for exciting the HEX molecules, and thus was chosen for the subsequenct fluorescence-based studies. 
In order to obtain quantitative information regarding the strength of fluorescence from each matrix, the captured fluorescent images (green light excitation) were subjected to the imaging analysis methodology described in Section 3.2.6.2 and shown in Figure 3.12b.

Figure 3.12b: Image analysis of HEX-labelled aptamer E6 (HEX-E6) binding to control and TCS E matrices

\begin{tabular}{|c|c|c|c|c|c|}
\hline $\begin{array}{l}\text { HEX- } \\
\text { E6 }\end{array}$ & $\begin{array}{l}\text { Fluorescent } \\
\text { image }\end{array}$ & $\begin{array}{l}\text { Black and } \\
\text { white image }\end{array}$ & $\begin{array}{l}\text { ROI } \\
\text { Beads }\end{array}$ & $\begin{array}{l}\text { ROI } \\
\text { Buffer }\end{array}$ & $\mathrm{FC}_{\text {mean }}$ \\
\hline $\begin{array}{l}\text { control } \\
\text { E } \\
\text { matrix }\end{array}$ & & & & & 10427 \\
\hline $\begin{array}{c}\text { TCS } \\
\text { E } \\
\text { matrix }\end{array}$ & & & & & 35552 \\
\hline
\end{tabular}

$\mathrm{ROI}=$ regions of interest; $\mathrm{FC}_{\text {beads }}=$ average fluorescent counts on beads; $\mathrm{FC}_{\text {buffer }}=$ average fluorescent counts on buffer and; $\mathrm{FC}_{\text {mean }}=$ mean fluorescent counts, which defined as $\mathrm{FC}_{\text {beads }}-\mathrm{FC}_{\text {buffer. }}$

After choosing regions of interest (ROI) on both the beads and buffer solution, average fluorescent counts (FC) within each ROI was then determined by the IGOR PRO software.

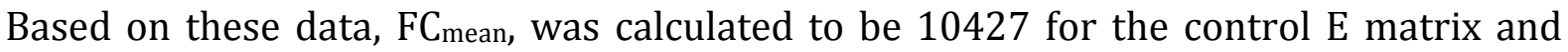
35552 for the TCS E matrix (Figure 3.12b). These values represented the strength of fluorescent intensity within each matrix, and were used to indicate binding affinities of HEX-E6 aptamer to TCS.

The fluorescent imaging method was applied to assess binding affinity of TCS R8 aptamers D4, D5, D6, E5 and E6. The fluorescent images captured from these experiments are summarised in Figure 3.13a and analysed to obtain $\mathrm{FC}_{\text {mean }}$ value for each image (data not shown). Thereafter, the $\mathrm{FC}_{\text {mean }}$ from an average of three independent experiments was plotted for each aptamer being tested (Figure 3.13b).

As shown in Figure 3.13a and 3.13b, aptamers D5 and E6 showed a higher level of binding to TCS-conjugated matrices than to control matrices, indicating that these two aptamers had higher affinities for TCS than D4, D6 and E5 aptamers. 
Figure 3.13a: Fluorescent images showing affinities of TCS round 8 (R8) aptamers D4, D5, D6, E5 and E6 to control and TCS-conjugated matrices

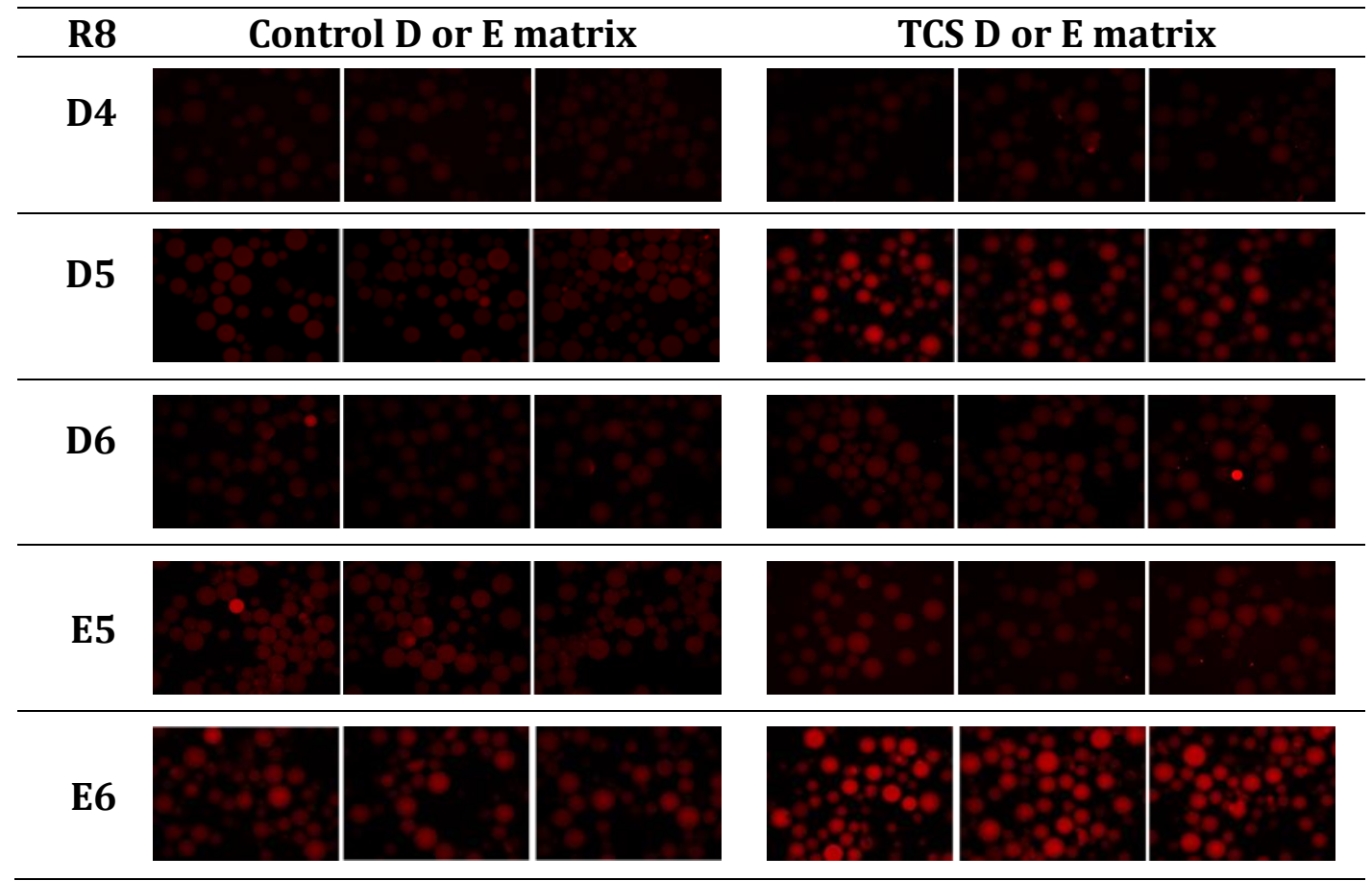

Images were captured under same conditions with 1 sec of exposure time via three independent experiments.

Figure 3.13b: Fluorescent counts representing binding affinities of TCS round 8 (R8) aptamers to control and TCS-conjugated matrices

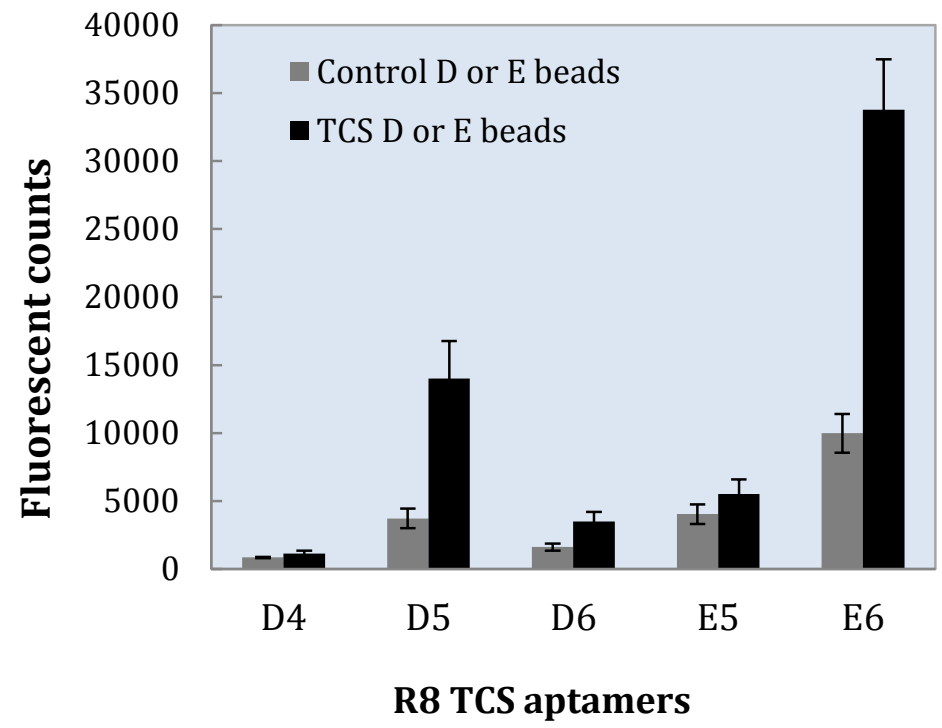

The error bars represent the standard deviation of fluorescent counts from three independent experiments. 
The fluorescent imaging method was also utilised to determine the $K_{d}$ value for aptamer D5. The resulting images from the $K_{d}$ experiment are summarised in Figure 3.14a, and then analysed to obtain $\mathrm{FC}$ mean value for each image (data not shown).

Figure 3.14a: Fluorescent images of HEX-labelled TCS aptamer D5 (HEX-D5) binding to control and TCS D matrices at 8 concentrations ranging from 40 to $1000 \mathrm{nM}$

\begin{tabular}{|c|c|c|c|c|c|c|c|c|}
\hline$(\mathrm{nM})$ & 1000 & 800 & 600 & 400 & 250 & 160 & 80 & 40 \\
\hline $\begin{array}{r}\text { D5- } \\
\text { control } \\
\text { D matrix }\end{array}$ & & & & & & & & \\
\hline $\begin{array}{r}\text { D5-TCS } \\
\text { D matrix }\end{array}$ & & & & & & & & \\
\hline
\end{tabular}

Each image was taken under same conditions with $1 \mathrm{sec}$ of exposure time.

Binding saturation curves for aptamer D5 against control and TCS D matrices were plotted (Figure 3.14b i). Subsequently, the mean \pm SD $K_{d}$ value for aptamer D5 was determined to be $584 \pm 251 \mathrm{nM}$ based on the normalised binding curve (Figure 3.14b ii).

Figure 3.14b: Saturation curves of (i) TCS aptamer D5 to control and TCS D matrices, and (ii) a normalised saturation curve of D5 by subtracting the non-specific binding to control matrix from the specific binding to TCS D matrix
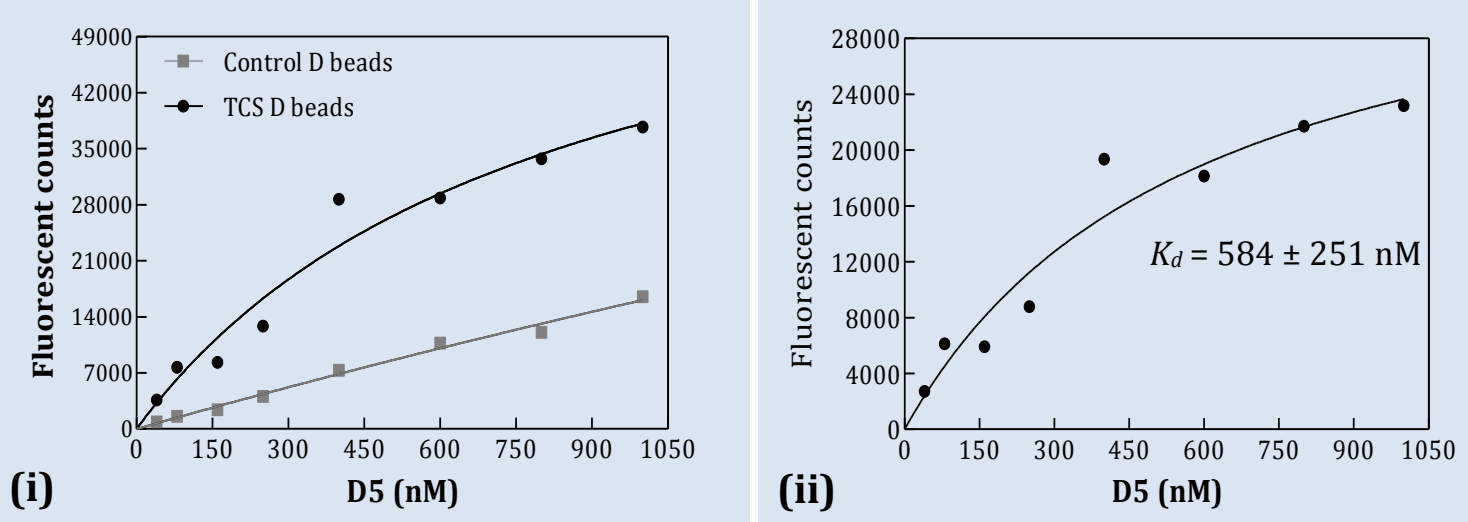

In the final experiments, HEX-D5 bound TCS D matrix and HEX-E6 bound TCS E matrix were each visualised after eluting both aptamers from the matrices in order to check if the elution steps used in the fluorescent binding assay preparation were capable of fully recovering aptamers D5 and E6 from the aptamer-bound matrices (Figure 3.15). 
Figure 3.15: Figure 3.15: Fluorescent images of HEX-labelled aptamers D5-bound TCS D matrix and E6-bound TCS E matrix after elution

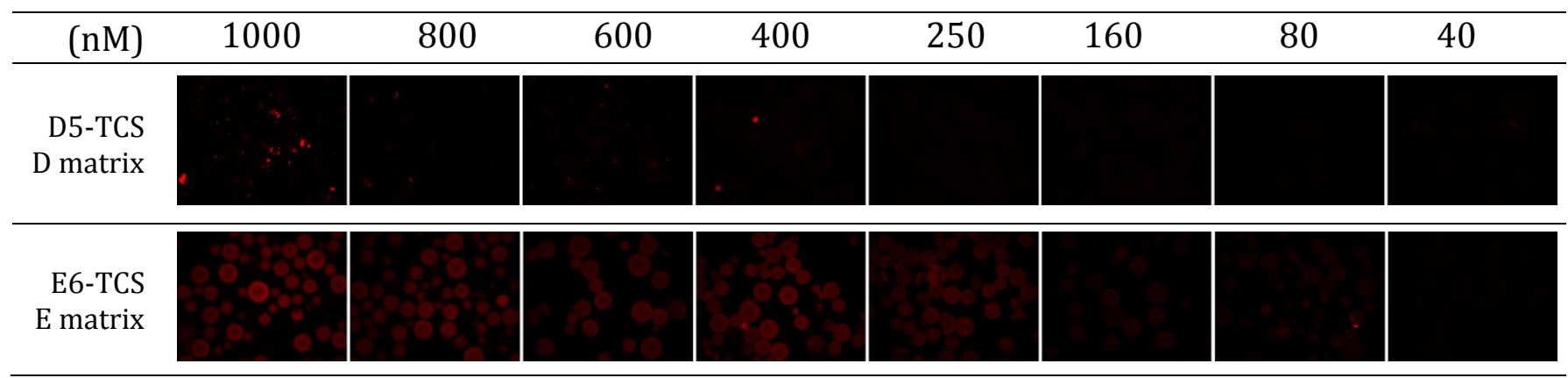

Each image was taken under same conditions with 1 sec of exposure time.

As shown in Figure 3.15, no observable fluorescent signals were left on HEX-D5 bound matrix. However, HEX-E6 bound matrix showed a relatively high fluorescent signals indicating either current elution conditions were not stringent enough to fully recover the aptamer E6, or that this aptamer was too strong to be eluted.

\subsection{Discussion}

The work in this chapter describes the generation of ssDNA aptamers capable of binding TCS using an affinity column-based SELEX strategy referred to as the basic-SELEX. A total of ten monoclonal TCS aptamers were generated after eight rounds of selection (Table 3.2). These aptamers are then subjected to a number of in silico characterisation, such as nucleotide sequence analyses, phylogenetic evolutionary relationship and 2D structure prediction. Discussion of these results will be made in general discussion (see Chapter 6).

The selection procedure was restricted to 8 rounds due to the formation of non-specific PCR by-products (generally larger than 75 bases) during conventional PCR amplification (Figure 3.4). The undesired PCR by-products are thought to be the consequence of over amplification. The amount of aptamers captured by each affinity matrix generally increases with increasing rounds of selection, thus requiring reduced numbers of PCR cycle amplification. However, PCR cycle number was kept constant in this study resulting in unnecessary cycles of amplification which can often lead to non-specific annealing of the template by primers, thereby forming PCR products with variable lengthes. 
The binding capability of TCS R8 aptamers to TCS was validated using the dot blot assay, and this method successfully allowed for the identification of the desired aptamer candidates to only two sequences (i.e. aptamers D5 and E6). Initial binding affinity studies revealed five aptamers (i.e. D4, D5, D6, E5 and E6) that had superior binding affinity, as the intensity of their dot blot signals were the strongest among all aptamers tested (Figure 3.9). At this stage, it was uncertain as to which of the five sequences would turn out to be the best TCS aptamer. With this in mind, these five aptamers were further examined for their binding specificity by testing their ability to recognise compounds (i.e. MTS, BPA, OE2 and DCF) sharing structural similarities with TCS. Three candidates D4, D6, and E5 showed higher levels of binding affinity to the compounds tested indicating they were less specific to TCS (Figure $3.10 \mathrm{i}$, iii and iv), thus they were excluded for further studies. Only two aptamers (D5 and E6) were considered suitable for further evaluation (Figure 3.10 ii and v).

The aforementioned experiments demonstrated the screening power of the dot blot assay. However, it is worth noting that this method utilises nitrocellulose membranes as a carrier for holding TCS. The membrane is generally designed to hold macromolecules such as proteins, and their ability to retain small molecules like TCS is unclear. As evidenced from the dot blot results, small molecules like TCS and other structurally similar compounds need to be prepared in large quantities, i.e. up to $50 \mathrm{mg} / \mathrm{mL}$, in order to get sufficient signals (Figure $3.10 \mathrm{i}-\mathrm{v}$ ). Additionally, the dot blot signals do not always follow a titration trend in signal strength corresponding to an increase in the amount of molecules being spotted onto the membrane. There were no observable signals when TCS was spotted at 150 and $75 \mathrm{nmol}$ (Figure 3.9). This is unlikely to be due to the low affinity of the aptamers being tested, as the intensity of the dot blot signals is not proportional to the amount of TCS being spotted even at large quantities. For example, the aptamers D6 and E6 showed weaker signals at $600 \mathrm{nmol}$ when compared with $300 \mathrm{nmol}$ (Figure 3.9). Therefore, it was more likely that TCS when spotted at low quantities completely dissociated from the membrane during washing steps, whereas some of the TCS molecules remain attached to the membrane when large quantities were spotted. The lack of signal titration is an issue when using the dot blot assay for aptamer characterisation. 
During SELEX experiments, $K d$ determination provides an indicator for the likely performance of selected aptamers. The fluorescent binding assay was utilised to determine the $K_{d}$ values of aptamers D5 and E6. These two aptamers demonstrated notable differences in their relative binding capacities to TCS (Figure 3.11 A and B). The mean \pm SD $K d$ value for the aptamer D5 was determined to be $700 \pm 174$ nM (Figure 3.11 C), and was comparable to the published $K_{d}$ values for PCBs (Mehta et al. 2012; Xu et al. 2012). In contrast, aptamer E6 under current assay conditions failed to reach saturation (Figure $3.11 \mathrm{D}$ ). Based on these results, aptamer D5 was considered to have the most avid binding to TCS with respect to its $K_{d}$ value. In addition, both aptamers displayed some non-specific binding to unconjugated matrices (Figure $3.11 \mathrm{~A}$ and $\mathrm{B}$ ). This result compromised their binding specificity to TCS. The observation of non-specific binding could be due to either non-specific electrostatic interaction between an aptamer and the surface of a matrix or specific recognition of the matrix by aptamers. As a consequence, the future practical use of aptamers D5 and E6 might be questionable unless a method can be found to eliminate their non-specific binding to the matrix.

One of the key steps in the basic-SELEX procedure is the conjugation of TCS to column matrix. As mentioned in the introduction, it was assumed that the long-arm matrix should have less steric hindrance upon aptamer binding, thus be more efficient in selecting TCS specific aptamers when compared with the short-arm matrix. This indeed was the case, as evidenced during the progression of the SELEX selection. After 6 rounds of selection, polyclonal aptamers generated using TCS D matrix showed apparent dot blot signals at 600 and $300 \mathrm{nmol}$, whereas the use of TCS E matrix required at least 8 rounds of selection to get decent dot blot signals (Figure $3.5 \mathrm{i}$ and ii). In addition, when screening the binding affinities of TCS R8 aptamers, TCS D aptamers, in general, showed more intense dot blot signals than TCS E aptamers (Figure 3.9). However, the most convincing evidence supporting the use of a long-arm matrix for selection came from the $K_{d}$ measurements (Figure 3.11 C and D). Aptamer D5 generated using the long-arm matrix showed lower a $K_{d}$ value than aptamer E6 which was produced by the short-arm matrix. Therefore, the long-arm TCS D matrix is suggested to be the preferred matrix for future selection. 
The development of a fluorescent imaging-based technique could potentially simplify the way to characterise aptamer binding properties. From this perspective, the fluorescent imaging assay was subjected to an initial "proof of concept" experiment with the use of aptamer E6. The experiment demonstrated that TCS-conjugated matrices can elicit fluorescent signals upon binding by the HEX-labelled aptamer. By evaluating the fluorescent intensity of each image, the method is capable of distinguishing a difference between aptamer-bound control matrix and TCS-conjugated matrix (Figure 3.12a and $3.12 \mathrm{~b}$ ) thus verifying that the aptamer E6 was specifically binding to TCS. In addition, binding affinity of aptamers D4, D5, D6, E5 and E6 were also screened using the fluorescent imaging method. From the earlier studies using the dot blot assay, it was established that these aptamers were capable of binding to TCS. The purpose for using the fluorescent imaging assay was to evaluate whether this method might be suitable for aptamer characterisation and for quantitating aptamer binding affinities. As shown in Figure 3.13a and 3.13b, aptamer D5 and E6 showed a higher level of binding to TCSconjugated matrices than to control matrices, indicating the two aptamers had higher affinities than the other aptamers. These results agreed with those obtained from the dot blot assay. Furthermore, the $K_{d}$ value of $584 \pm 251 \mathrm{nM}$ for aptamer D5 determined using the imaging method (Figure 3.14b ii) was comparable to that obtained from the elutionbased method $\left(K_{d}=700 \pm 174 \mathrm{nM}\right)$.

It is noteworthy that the standard deviation of the $K_{d}$ from the imaging method is relatively large indicating that more data points are required to minimise the variation. However, further experiments were not carried out, since results from both the dot blot and fluorescent binding assays showed that most of TCS R8 aptamers had either low affinity (e.g. D1, D2, D3, E1 and E3) or poor specificity (e.g. D4, D6 and E5). From the remaining two aptamers (i.e. D5 and E6), there was evidence of high levels of non-specific binding. Therefore, it was considered that none of the R8 aptamers were ideal candidates to be used for thoroughly investigating the potential usages of the fluorescent imaging method. Finally, the imaging method also revealed that the elution steps performed in the fluorescent binding assay procedure was unable to fully remove the bound-aptamer E6 (Figure 3.15), thus compromising the accuracy of using this method for $K_{d}$ determination. To sum up, these preliminary studies demonstrated that the fluorescent imaging method provided a simple-to-use assay for aptamer characterisation. 


\subsection{Conclusion}

Based on the work presented here, TCS specific aptamers were generated and characterised with acceptable binding affinity and specificity to the target molecule. Aptamer D5 was proven to be the best candidate with a $K_{d}$ value of $700 \mathrm{nM}$ similar to some of the published $K_{d}$ values for structurally similar molecules. The long-arm TCS D matrix proved to be more effective than the short-arm matrix in generating aptamers with better quality. Aptamer characterisation by dot blot and fluorescent binding assays have shown some promising results in the basic-SELEX study, despite each of them has its own limitations. In contrast, the fluorescent imaging assay explored in this chapter can potentially solve these problems, as this method not only avoids the use of nitrocellulose membrane, but also eliminates the need to elute bound aptamers. Furthermore, this method alone can potentially provide a comprehensive methodology for aptamer characterisation including the determination of $K_{d}$ values. Finally, the basic-SELEX procedure was found to have limitations in generating TCS specific aptamers as evidenced by the observation of non-specific binding to the surface of a matrix by aptamers. Besides, the selection process was restricted from going beyond eight rounds due to the formation of non-specific PCR by-products.

Overall, it was concluded that aptamers generated during the basic-SELEX procedure are unsuitable for applications in aptamer-based biosensors for TCS detection. To advance the utility of TCS aptamers for biosensing, it is suggested that the basic-SELEX procedure needs to be modified in order to generate aptamers with higher affinity and better specificity to TCS. 


\section{CHAPTER 4 \\ TRICLOSAN APTAMER SELECTION: REVISING THE SELEX METHOD}

\subsection{Introduction}

The basic-SELEX procedure was found to have limitations in generating TCS aptamers with high affinity and specificity (see Chapter 3). These included: [i] non-specific binding to the matrix by aptamers; [ii] the lack of capability for conventional PCR to specifically amplify the selected aptamer sequences and; [iii] limitations when using dot blot and fluorescent binding assays for aptamer characterisation. To mitigate these problems, a few modifications were introduced to the selection process, and a revised-SELEX procedure was investigated in this chapter aiming to generate aptamers with improved binding properties. These modifications involved: [i] the use of transfer RNA (tRNA) formulated Blotto for matrix blocking; [ii] the inclusion of negative and counter selection strategies; [iii] replacing conventional PCR with real-time PCR for amplification and; [iv] utilising a fluorescent imaging assay for aptamer characterisation.

To minimise electrostatic interactions between oligonucleotides and the matrix, a new Blotto solution was prepared by adding tRNA to the old Blotto solution formulated using skim milk powder. Being composed of nucleic acids, it was postulated that tRNA would be more appropriate for reducing non-specific adsorptions caused by oligonucleotides when compared with the milk, as the latter is often effective in reducing non-specific binding by proteins. The presence of tRNA in large quantities can potentially restrict the access of oligonucleotides to those polar-regions presented on the surface of a matrix (Lin and McNatty 2009). This new blocking strategy was applied for blocking all matrices.

During earlier studies, it became increasingly evident that any aptamer sequences with non-specific binding properties towards matrix and/or linker arm needed to be excluded when performing an affinity column based-SELEX procedure. This is because aptamers are normally used to measure free target in solution. Thus, any non-specific affinity to the matrix is likely to reduce the functionality of an aptamer in sensing applications. 
For example, the aptamer for rhodamine displayed a weaker binding to rhodamine when in solution compared to when it was immobilised on a matrix (Wilson and Szostak 1998). To eliminate sequences that display binding or partial recognition to the matrix, SELEX experiments sometimes included a negative selection step with unconjugated matrix before a positive round of selection with the target-immobilised matrix (Jayasena 1999). In addition, to further enhance aptamer selectivity, it is common to perform selections with compounds having similar molecular structures to the target molecule (Jenison et al. 1994). This selection strategy is referred to as counter selection, as it is used to remove any sequences from the pool that may have affinity to structurally similar molecules, which might well be found in samples containing the target of interest. Therefore, both negative and counter selection strategies were introduced for aptamer selection.

Conventional PCR was replaced by a real-time PCR in the revised-SELEX procedure to amplify aptamer-bound matrices, as the levels of fluorescent signal from the real-time PCR can be used to follow the amplification process. Once sufficient amplification is reached, the reaction can be manually stopped to avoid over-amplification, thus reducing the likelyhood of forming any undesired PCR by-products. The fluorescent imaging assay developed in Chapter 3 was the method of choice for aptamer characterisation, as the dot blot and fluorescent binding assays used in the basic-SELEX procedure showed some limitations. For example, aptamer characterisation by dot blot assay can only provide qualitative measures regarding binding affinity and specificity of an aptamer. The lack of quantitation restricts the use of this assay for $K_{d}$ determination. In the fluorescent binding assay, an elution step is inevitable in order to get quantitative information. The efficiency of this elution step is not easy to measure routinely. Thus, more often than not, it is assumed that no detectable fluorescent signals in the eluates means no aptamers left over on the matrix. As demonstrated in Chapter 3, this is not always the case since the E6 aptamer showed incomplete elution from the aptamer-bound matrix.

In addition, aptamers generated using the revised-procedure were subjected to insolution characterisation of the target binding activity using CD spectroscopy, as conformational change induced by aptamer-target interactions forms the foundation for many biosensing approaches (Levy et al. 2005; Liu and Lu 2006; Liu et al. 2011). 
Morover, truncation of aptamer sequences based on in silico characterisation can have a profound effect on the target binding ability of aptamers. Lin and McNatty demonstrated that the target binding characteristics of an aptamer can be improved when specific regions of the aptamer identified from the predicted 2D structure were utilised in Eastern blotting assay (Lin and McNatty 2009). The LOD was significantly improved when using the selected regions instead of the parent aptamer as the probing agent for the assay. In addition, Alsager et al. reported an OE2 aptasensor showed an increased $L O D$ when using truncated versions of the OE2 aptamer based on the 2D structural analysis (Alsager et al. 2015). The applicability of the truncation method to TCS-specific aptamers is yet to be determined. Furthermore, in employing the revised-SELEX procedure, only the long-arm TCS D matrix was investigated, as the evidence from Chapter 3 suggesting it was more suitable for aptamer selection when compared with the short-arm TCS E matrix.

To sum up, the objectives of this chapter were to: [i] assess the levels of improvement in aptamer selection using the aforementioned SELEX modifications; [ii] investigate the performance of the fluorescent imaging assay; [iii] select TCS aptamers with improved affinity and specificity as assessed by their $K_{d}$ values and in-solution binding studies and; [iv] investigate the efficacy of truncated sequences of the best TCS aptamer.

\subsection{Methods}

Apart from the aforementioned SELEX-modifications, all other steps in the revised-SELEX procedure were performed as described for the basic-SELEX procedure (see Section 3.2, Chapter 3). The following method section describes how the modifications were incorporated into the selection process for generating TCS-specific aptamers.

\subsubsection{Synthesis of affinity matrices}

Preparation of the TCS D matrix is described in Chapter 2 (see Section 2.1.1). Three other matrices were also included in the revised-SELEX procedure, namely control D matrix, BPA- and OE2-matrices. The control D matrix synthesised during the preparation of the TCS D matrix was used for negative selection. The BPA- and OE2-matrices synthesised as described in Chapter 2 (see Section 2.1.1) were used for counter selections. 


\subsubsection{Revised-SELEX procedure}

The N40 aptamer library and primer sequences used in the revised-SELEX procedure were the same as the basic-SELEX procedure, except for the forward primer was labelled with a HEX tag, and it was incorporated to the forward strands of dsDNA during amplification, thereby providing aptamers with fluorescence capability. Primer combinations used in the revised-SELEX procedure are summarised in Table 4.1.

Table 4.1: Primer combinations used at different stages of the revised-SELEX procedure

\begin{tabular}{lll}
\hline Stage & Forward & Reverse \\
\hline Selection & HEX-Pf & biotin-Pr \\
Cloning and sequencing & Pf & Pr \\
\hline
\end{tabular}

$\mathrm{Pf}=$ forward primer and; $\mathrm{Pr}=$ reverse primer.

Preparations of all synthesised oligonucleotides and buffers were carried out as outlined in Chapter 2 (see Section 2.3). The following section provides a summary of the revisedSELEX procedure for selecting TCS-specific aptamers.

\subsubsection{Blocking of affinity matrices}

A new Blotto solution prepared by adding tRNA to the Blotto solution at $10 \mathrm{mg} / \mathrm{mL}$ was utilised to block all matrices (i.e. control and TCS D matrices, as well as BPA- and OE2matrices). The process for washing and blocking these matrices was performed as described in Chapter 2 (see Section 2.4.1).

\subsubsection{Denaturation of ssDNA aptamer library}

The initial synthetic aptamer library ( $3 \mathrm{nmol}$ ), and those libraries (each $\sim 50 \mathrm{pmol}$ ) used for subsequent rounds of selection were subjected to a heat-denaturing process (see Section 2.4.2, Chapter 2) before being incubated with either TCS D matrix for positive selections or appropriate matrices for negative and counter selection. 


\subsubsection{Co-incubation of the aptamer library with the affinity matrices}

For the first two cycles, the aptamer library for each cycle was only incubated with TCS D matrix. Before starting the third cycle, a negative selection was performed by incubating the aptamer library with control D matrix for $60 \mathrm{mins}$ at RT with gentle shaking. Then, the aptamer-control matrix suspension was centrifuged for 2 mins at $13,000 \times \mathrm{g}$. Thereafter, the supernatant was collected and added to TCS D matrix to start the third round of selection. Counter selections using OE2- and BPA-matrices were performed before selection rounds 6 and 8, respectively. These selections were performed as described for the negative selection.

\subsubsection{Removal of unbound oligonucleotides}

Following incubation with TCS D matrix, sequences with little or no affinity for TCS were removed during multiple washing steps (see Section 2.4.4, Chapter 2). The resulting aptamer-bound matrices as well as those obtained from negative and counter selections were subjected to real-time PCR amplification.

\subsubsection{Real-time PCR amplification}

A real-time PCR sample was prepared as for a standard PCR sample (see Section 2.4.5, Chapter 2), except for the addition of SYBR Green (1X final concentration), and the use of different primer combinations (Table 4.1). Furthermore, the real-time amplification process was stopped immediately after the signal output reached a plateau, as assessed by SYBR green binding to dsDNA molecules. Following amplification, the real-time PCR products from positive, negative and counter selections were purified and visualised (see Section 2.4.6, Chapter 2). Only the $75 \mathrm{bp}$ bands from positive selections were excised, extracted and strand-separated as described in Chapter 2 (see Section 2.4.6-8).

\subsubsection{Selection conditions for the revised-SELEX procedure}

Ten cycles of positive selections were undertaken which involved one negative selection and two counter selection steps in the revised-SELEX procedure. The stringency of selection was controlled in a similar fashion as for the basic-SELEX by altering the amount of TCS D matrix used, the time of incubation and the volume of washing within each cycle (Table 4.2). 
Table 4.2: Aptamer selection conditions used in the revised-SELEX procedure

\begin{tabular}{cccccc}
\hline $\begin{array}{c}\text { SELEX } \\
\text { round }\end{array}$ & $\begin{array}{c}\text { Affinity column } \\
(\mathbf{m g})\end{array}$ & $\begin{array}{c}\text { Incubation } \\
\text { time (hr) }\end{array}$ & $\begin{array}{c}\text { Incubation } \\
\left.\text { temperature } \mathbf{(}^{\circ} \mathbf{C}\right)\end{array}$ & $\begin{array}{c}\text { Wash volume } \\
(\mathbf{m L})\end{array}$ \\
\hline 1 & TCS & 50 & 12 & 4 & 5 \\
2 & TCS & 40 & 12 & 4 & 5 \\
$\mathbf{3}$ & Control & $\mathbf{2 0}$ & $\mathbf{1}$ & $\mathbf{2 3}$ & $\mathbf{n} / \mathbf{a}$ \\
& TCS & $\mathbf{3 0}$ & $\mathbf{1 2}$ & $\mathbf{4}$ & $\mathbf{1 0}$ \\
4 & TCS & 30 & 8 & 4 & 15 \\
5 & TCS & 30 & 8 & 4 & 20 \\
$\mathbf{6}$ & OE2 & $\mathbf{2 0}$ & $\mathbf{1}$ & $\mathbf{2 3}$ & $\mathbf{n} / \mathbf{a}$ \\
& TCS & $\mathbf{2 0}$ & $\mathbf{8}$ & $\mathbf{4}$ & $\mathbf{2 5}$ \\
7 & TCS & 20 & 4 & 12 & 30 \\
$\mathbf{8}$ & BPA & $\mathbf{2 0}$ & $\mathbf{1}$ & $\mathbf{2 3}$ & $\mathbf{n} / \mathbf{a}$ \\
& TCS & $\mathbf{2 0}$ & $\mathbf{4}$ & $\mathbf{1 2}$ & $\mathbf{3 5}$ \\
9 & TCS & 10 & 1 & 4 & 40 \\
10 & TCS & 10 & 1 & 4 & 45 \\
\hline
\end{tabular}

The bold represents negative and counter selections undertaken at the respective SELEX round.

\subsubsection{Progression of the revised-SELEX procedure}

The fluorescent imaging assay was utilised to assess the progression of the revised-SELEX procedure. In brief, $5 \mu \mathrm{L}$ of aptamer-bound TCS D matrix from each cycle was resuspended in $25 \mu \mathrm{L}$ of $1 \mathrm{X}$ BWB, and the resulting matrix suspension was loaded onto a glass slide with a cover slip on top. Thereafter, the glass slide was visualised using fluorescent microscopy. For each cycle, three images were captured corresponding to three different regions of the slide under blue light excitation. The captured images were analysed using the methodology described in Chapter 3 (see Section 3.2.6.2). Enrichment of the aptamer library was assessed at each selection round by monitoring average fluorescent signals from the aptamer-bound TCS D matrix via the measurement of three images per round. The selection process was stopped when no increase in fluorescence was observed. 


\subsubsection{DNA cloning, sequencing and nucleotide analysis}

Following the fluorescent imaging assay, and provided no increase in fluorescence was observed, the selected aptamer library from round 10 was cloned and sequenced to identify their nucleotide compositions as outlined in Chapter 2 (see Section 2.6.1- 5). Ten unique sequences (each 75 bases), referred to as TCS round 10 (R10) aptamers, were identified from the sequencing step. Thereafter, nucleotide analyses of TCS R10 aptamers were performed as described in Chapter 2 (see Section 2.6.6).

\subsubsection{Aptamer characterisation}

The binding affinity and specificity of TCS R10 aptamers were characterised. In addition, their $K_{d}$ values, as well as the degree of conformational change in-solution were detemined. All aptamers being characterised were synthesised without labelling (CD spectroscopy), or synthesised and modified at the 5 '-end with either a biotin tag (fluorescent binding assay) or HEX tag (fluorescent imaging assay). Furthermore, a 75 base-long random sequence synthesised and modified the same as aptamers was included as a control to assess non-specific binding of oligonucleotides during the following characterisation process.

\subsubsection{Binding affinity}

The fluorescent imaging assay was utilised to screen binding affinity of TCS R10 aptamers. The assays were performed as described in Chapter 3 (see Section 3.2.6.1), except that: [i] control and TCS D matrices were utilised for binding with aptamers; [ii] each aptamer being tested was prepared at a final concentration of $150 \mathrm{nM}$; [iii] random sequence (75 mer) was included as a control and; [iv] green light was utilised to excite the fluorophore. The fluorescent images captured from both control and TCS D matrices were then analysed using the methodology described in Chapter 3 (see Section 3.2.6.2).

\subsubsection{2 $K_{d}$ Determination using both fluorescent imaging and binding assays}

The $K_{d}$ values of TCS R10 aptamers were determined firstly using the fluorescent imaging assay, and secondly using the fluorescent binding assay under identical conditions to establish if the imaging method was suitable for $K_{d}$ determination. Preparation of aptamer-bound matrices for the $K_{d}$ experiments was performed as described in Chapter 2 (see Section 2.7.2) with modifications: 
[i] the use of control and TCS D matrices for binding with aptamers tested; [ii] each aptamer was prepared at a final concentration range of 20-750 nM; [iii] in the imaging method, aptamer-bound matrices were visualised under the microscope, whereas in the affinity procedure, aptamer-bound matrices were subjected to elution and; [iv] random sequence was included in both assays as a control, and was prepared the same as each aptamer tested.

The fluorescent images captured from the imaging method were analysed as described in Chapter 3 (see Section 3.2.6.2), and the binding saturation curve for each sequence tested was generated by plotting the concentrations of assayed sequences versus their fluorescent counts following image analyses. The data obtained from the fluorescent binding assay was analysed as described in Chapter 2 (see Section 2.7.2), and the binding saturation curve for each sequence tested was generated by plotting the amount of eluted aptamer versus the concentration of the incubated aptamer. The $K_{d}$ values from both methods were determined using the GraphPad Prism software as described in Chapter 2 (see Section 2.7.2).

\subsubsection{In-solution conformational change}

The method for obtaining CD spectra is outlined in Chapter 2 (see Section 2.7.3). In brief, both the random sequence and TCS R10 aptamers with preferred binding properties were prepared at final concentration of $1 \mu \mathrm{M}$ with $1 \mathrm{X}$ BWB containing $5 \%(\mathrm{v} / \mathrm{v})$ ethanol, whereas TCS was prepared in the same buffer at concentrations of 2, 5 and $10 \mu \mathrm{M}$. The CD spectra of each tested sequence were recorded before and after mixing with TCS for 60 mins.

\subsubsection{Binding specificity}

The binding specificity of TCS R10 aptamers with the most desirable binding properties were investigated using the fluorescent imaging assay applied via an in-solution competitive binding strategy. The rationale here was that free TCS or structurally similar molecules in solution will compete with the immobilised TCS (i.e. on the matrix) for aptamer binding sites and cause matrix bound-aptamers to dissociate, thereby reducing the fluorescent signal of the aptamer-bound matrix. 
In brief, HEX-labelled aptamer aliquots (each $100 \mu \mathrm{L}$ ) were prepared using 1X BWB at $1200 \mathrm{nM}$, and each aliquot was heat-denatured (see Section 2.4.2, Chapter 2). TCS D matrix aliquots (each 1mg) were washed and blocked (see Section 2.4.1, Chapter 2). Thereafter, each aptamer aliquot was incubated with each matrix aliquote for 60 mins at RT. The resulting aptamer-bound matrix preparations were washed two times with 200 $\mu \mathrm{L}$ of ethanol-treated BWB (i.e. 1X BWB containing $5 \%$ ethanol). Following matrix washing, each aptamer-bound matrix preparation was resuspended in $200 \mu \mathrm{L}$ of the ethanol-treated BWB containing one of the competitive reagents (i.e. TCS, MTS, BPA and OE2) each prepared at $10 \mu \mathrm{M}$. A reference sample containing only ethanol-treated BWB was also prepared as a control. Thereafter, each matrix suspension was incubated at RT for 30 mins with gentle shaking. Following incubation, two washings using $200 \mu \mathrm{L}$ ethanol-treated BWB was carried out, and the resulting matrices were each resuspended in $30 \mu \mathrm{L}$ ethanol-treated BWB before being visualised under the fluorescent microscope. The extent of the reduction in the fluorescent intensity was used to evaluate the degree of non-specific interaction with each competitive reagent.

\subsubsection{Aptamer truncation study}

In order to locate the target binding site and evaluate the effect of different regions (i.e. primer and random region) of an aptamer on its target binding capability, TCS R10 aptamers with the most desirable binding properties were truncated by either removing forward, reverse or both primer regions. The resulting truncated versions of the aptamer (Table 4.3) were chemically synthesised and modified with a HEX tag at the 5'-end, and subjected to $K_{d}$ determination using the fluorescent imaging assay (see Section 4.2.5.2).

Table 4.3: Nucleotide sequences of the truncated TCS aptamer H6 with their given identities (IDs): 57H6F, 58H6R and 40H6B

\begin{tabular}{clc}
\hline ID & \multicolumn{1}{c}{ Sequence (5' - 3') } & Length \\
\hline $\mathbf{5 7 H 6 F}$ & GTCTGAAGGGATTGAGTATCCGGGGAGATGATGTCGTTGGTGAT & 57 \\
& AGTAAGAGCAATC & \\
$\mathbf{5 8 H 6 R}$ & $\begin{array}{l}\text { ATACGAGCTTGTTCAATAGTCTGAAGGGATTGAGTATCCGGGGA } \\
\text { GATGATGTCGTTGG }\end{array}$ & 58 \\
$\mathbf{4 0 H 6 B}$ & GTCTGAAGGGATTGAGTATCCGGGGAGATGATGTCGTTGG & 40 \\
\hline
\end{tabular}

The table shows sequences of 57H6F (forward primer deletion), 58H6R (reverse primer deletion), and 40H6B (both primer deletion); the red and blue highlighted nucleotides represent forward and reverse primer regions of $\mathrm{H} 6$ aptamer respectively. 


\subsection{Results}

\subsubsection{Preparation of affinity matrices}

Conjugation of BPA onto the surface of sepharose beads was confirmed by obtaining UVvisible absorption spectra of free BPA and BPA-matrix in water (Figure 4.1). Conjugation and characterisation of OE2-matrix were performed by Dr Shalen Kumar (data not shown).

Figure 4.1: UV-visible spectra of (A) free BPA in water and (B) BPA-matrix in water

A.

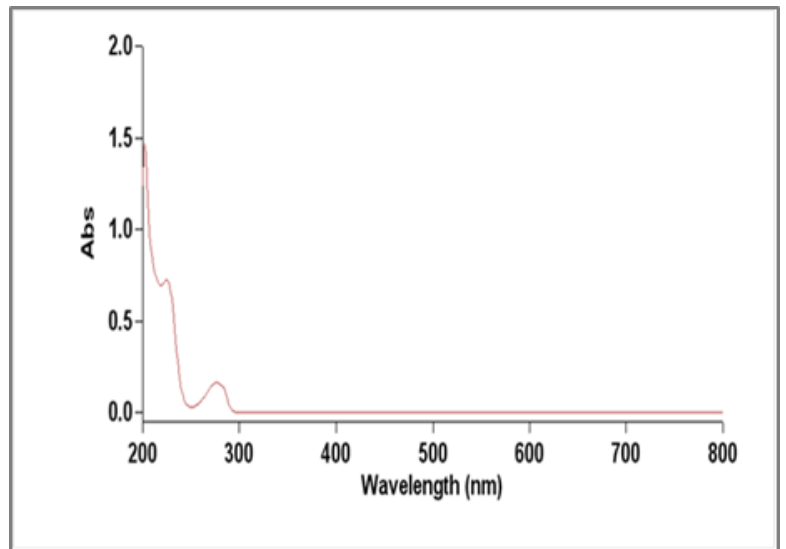

B.

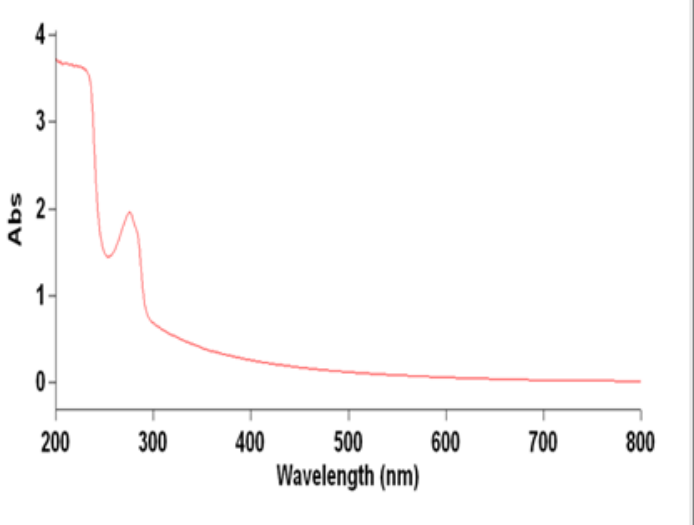

The presence of absorption peak at wavelengths 276 was observed for both BPAconjugated matrix and BPA in water thus confirming the successful conjugation of BPA onto the matrix.

\subsubsection{Elucidating the impact of negative and counter selections}

The effect of the negative and counter selections in the revised-SELEX procedure was assessed based on the band intensity of the amplified PCR products. Specifically, the aptamer-bound positive selection matrices (from rounds 3, 6 and 8), the negative selection matrix before round 3, and the counter selection matrices before rounds 6 and 8 were amplified under identical conditions using real-time PCR. Thereafter, the PCR products from these amplifications were subjected to agarose gel electrophoresis and visualised under UV light (Figure 4.2). 
Figure 4.2: Real-time PCR amplification of aptamer-bound positive, negative and counter selection matrices from the revised-SELEX rounds 3,6 , and 8

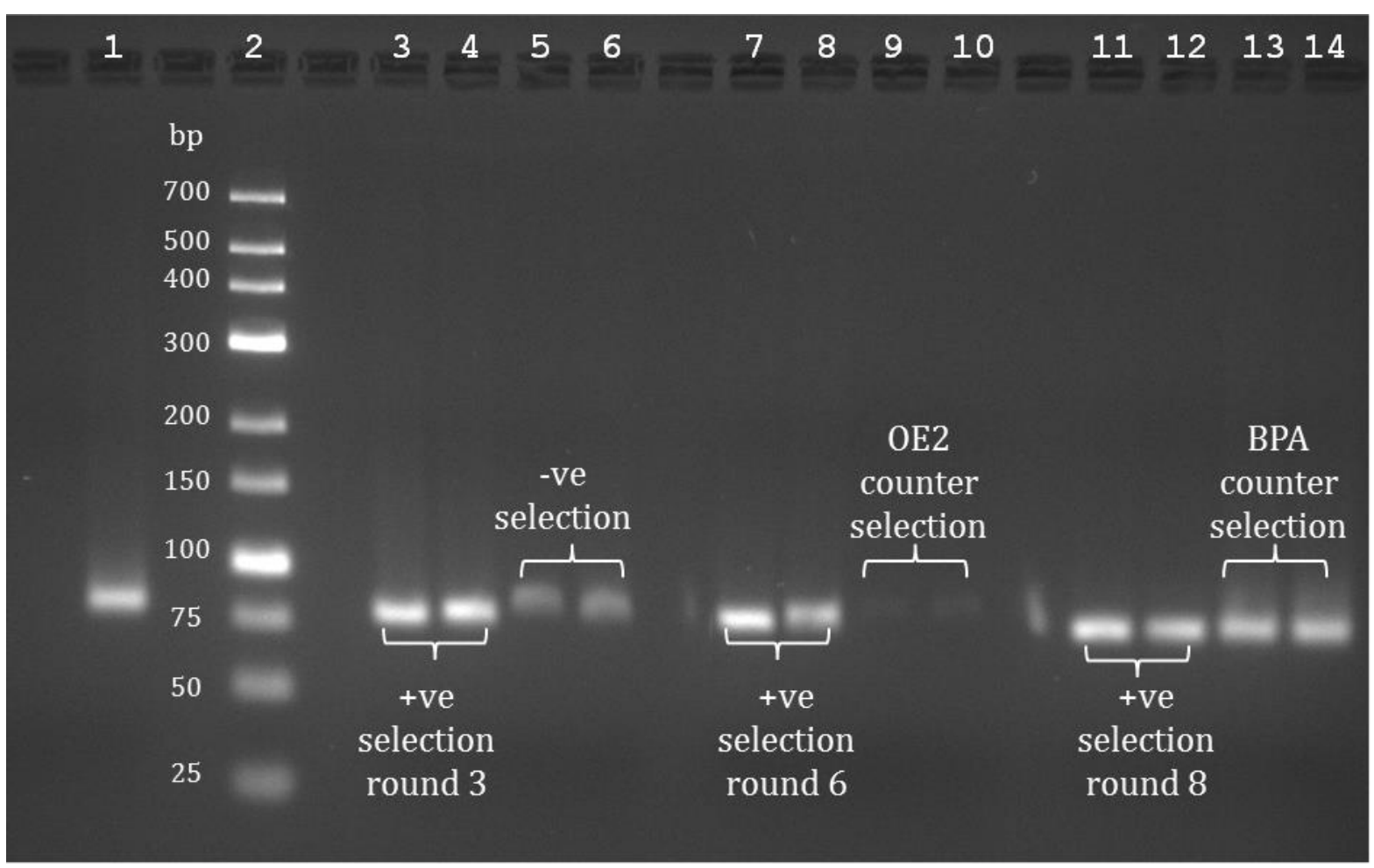

Lane 1: +ve control (1 nM N40 library); lane 2: DNA ladder; lanes $3 \& 4,7 \& 8$, and 11 \&12: the amplification of positive selection matrices from selection rounds 3,6 and 8 , respectively; lanes 5 \& 6: the amplification of unconjugated matrix for negative selection; lanes $9 \& 10$, and $13 \& 14$ : the amplification of OE2- and BPA-matrices respectively for counter selections; $\mathrm{bp}=$ base pair; $\mathrm{BPA}=$ bisphenol $\mathrm{A}$ and; $\mathrm{OE} 2=17-\beta$ oestradiol.

No non-specific PCR by-products formed after 8 rounds of positive selection (Figure 4.2, lanes 11 and 12). The intensity of 75 bp bands (Figure 4.2, lanes 5\&6, 9\&10, and 13\&14) were analysed to estimate the amount of oligonucleotide sequences removed from the aptamer pool by negative and counter selections.

\subsubsection{Progression of the-revised SELEX procedure}

To monitor the efficiency of the revised-SELEX procedure and to establish when the SELEX cycle can be stopped, the aptamer-bound TCS D matrix from each cycle was subjected to the fluorescent imaging assay (Figure 4.3a). 
Figure 4.3a: Fluorescent images of the aptamer-bound TCS D matrix from each revisedSELEX round

\begin{tabular}{|c|c|c|c|c|c|}
\hline Round & 1 & 2 & 3 & 4 & 5 \\
\hline Round & 6 & 7 & 8 & 9 & 10 \\
\hline
\end{tabular}

Fluorescent intensity of the aptamer-bound matrix at each SELEX round was analysed to quantify TCS binding affinity of each selected aptamer library (Figure 4.3b).

Figure 4.3b: Fluorescent counts representing the binding affinity of selected aptamer library at each revised-SELEX round to TCS D matrix

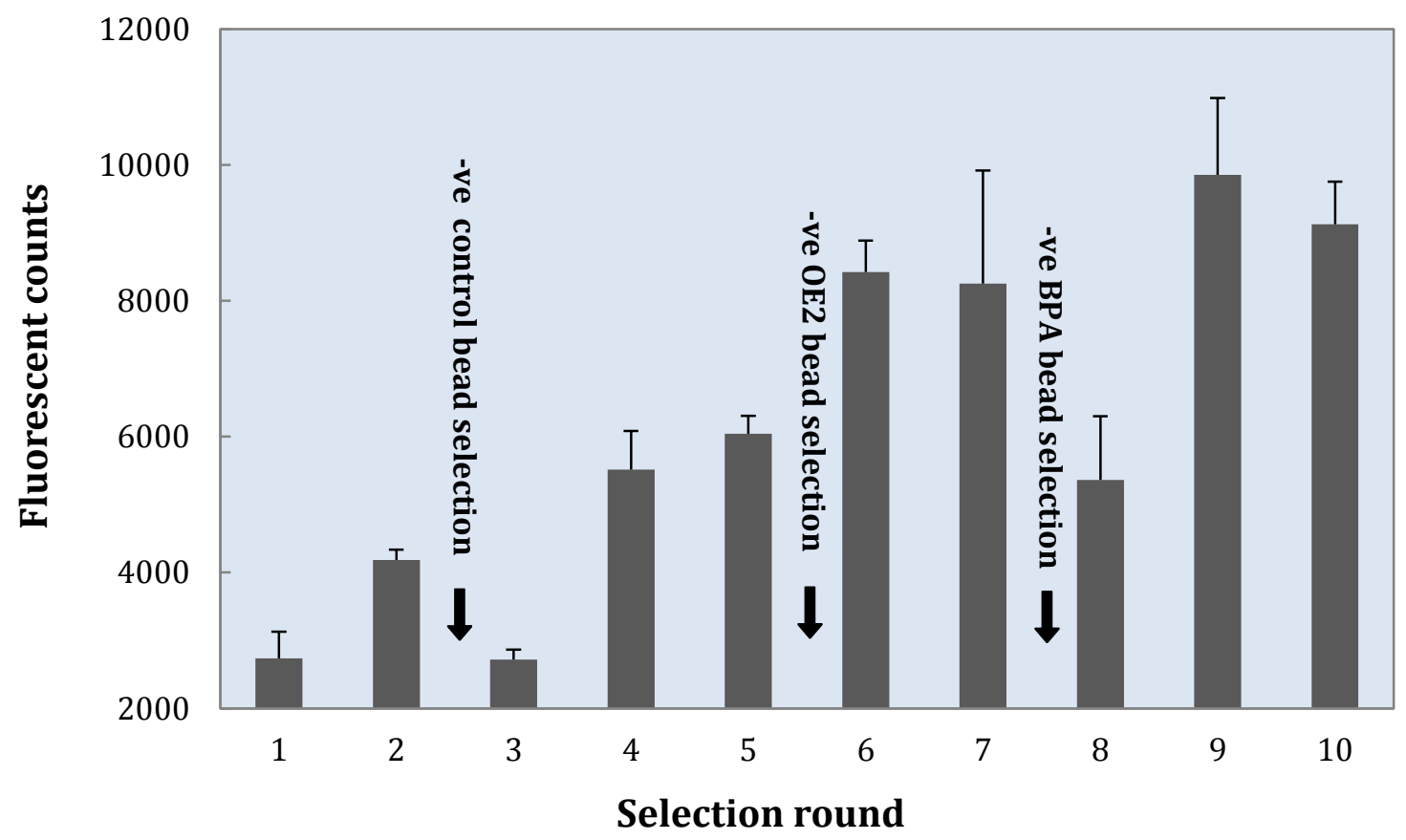

The error bars represent the standard deviation of the fluorescent counts measured from three separate images at each SELEX round.

As shown in Figure 4.3a and 4.3b, negative selections using control D matrix before round 3 showed a reduction in the amount of aptamer recovered indicating the removal of oligonucleotide sequences that non-specifically interact with the matrix surface. 
In contrast, there was no signal reduction when using OE2-matrix for counter selection before round 6. However, counter selection using BPA-matrix caused a significant reduction in the amount of aptamer recovered, and therefore indicationg the eliminating of oligonucleotide sequences capable of binding to BPA. Over all, no significant enrichment occurred at $9^{\text {th }}$ and $10^{\text {th }}$ rounds. Therefore, selection experiments were stopped after 10 cycles.

\subsubsection{DNA cloning, sequencing, and nucleotide analysis}

Ten monoclonal TCS aptamers (named as $\mathrm{H} 1-\mathrm{H} 10$ ) from the $10^{\text {th }}$ round (R10) of the revised-SELEX procedure were cloned and sequenced (Table 4.4).

Table 4.4: Nucleotide sequence of revised-SELEX round 10 TCS aptamers (H1 - H10) and the composition (\%) of individual bases (A, T, G and C) in the $\mathrm{N} 40$ random region within each aptamer

\begin{tabular}{clcccc}
\hline Clone & \multicolumn{1}{c}{ Full sequence 5' to 3' } & A (\%) & T (\%) & G (\%) & C (\%) \\
\hline $\mathbf{H 1}$ & $\begin{array}{l}\text { ATACGAGCTTGTTCAATACATTGCGGCGAGTGATGAGG } \\
\text { TTCGAAGGGGTGGGGGCGGGTGATAGTAAGAGCAATC }\end{array}$ & 15 & 17.5 & 55 & 12.5 \\
\hline $\mathbf{H 2}$ & $\begin{array}{l}\text { ATACGAGCTTGTTCAATACGGGGGAGGTGGGTTAAGG } \\
\text { GGGTGGAGAGTGTAGGTGTG TGATAGTAAGAGCAATC }\end{array}$ & 15 & 20 & 60 & 5 \\
\hline $\mathbf{H 3}$ & $\begin{array}{l}\text { ATACGAGCTTGTTCAATAGACGTTTGGGTCAGGTGGGT } \\
\text { GGTGCGGGGACGGTGTGTGGTGATAGTAAGAGCAATC }\end{array}$ & 7.5 & 25 & 57.5 & 10 \\
\hline $\mathbf{H 4}$ & $\begin{array}{l}\text { ATACGAGCTTGTTCAATAGGGTGGGGAGGTTAAAGGGC } \\
\text { CGGGGGTGGTGTCAGTTGTGTGATAGTAAGAGCAATC }\end{array}$ & 12.5 & 22.5 & 55 & 10 \\
\hline $\mathbf{H 5}$ & $\begin{array}{l}\text { ATACGAGCTTGTTCAATAGTTGGTAGGATGTTTGGACG } \\
\text { GAAGGGATGGGGGCTGGTGGTGATAGTAAGAGCAATC }\end{array}$ & 15 & 25 & 55 & 5 \\
\hline $\mathbf{H 6}$ & $\begin{array}{l}\text { ATACGAGCTTGTTCAATAGTCTGAAGGGATTGAGTATC } \\
\text { CGGGGAGATGATGTCGTTGGTGATAGTAAGAGCAATC }\end{array}$ & 20 & 27.5 & 42.5 & 10 \\
\hline $\mathbf{H 7}$ & $\begin{array}{l}\text { ATACGAGCTTGTTCAATACGGGTGGTTGAGGTGGGGCG } \\
\text { GGTTGGTGGAGGTTGTGGGGTGATAGTAAGAGCAATC }\end{array}$ & 5 & 25 & 62.5 & 7.5 \\
\hline $\mathbf{H 8}$ & $\begin{array}{l}\text { ATACGAGCTTGTTCAATATGTGATTGTGGATGTTGGGT } \\
\text { GTGATGGTTTTGGGCTGTGGTGATAGTAAGAGCAATC }\end{array}$ & 7.5 & 42.5 & 47.5 & 2.5 \\
\hline $\mathbf{H 9}$ & $\begin{array}{l}\text { ATACGAGCTTGTTCAATATCGGGCGGGTGGACGTGGTG } \\
\text { AGTTGGGGCGTTGTGGTGGGTGATAGTAAGAGCAATC }\end{array}$ & 5 & 25 & 57.5 & 12.5 \\
\hline $\mathbf{H 1 0}$ & $\begin{array}{l}\text { ATACGAGCTTGTTCAATATGGTCGTGGGGGAGGTGGTG } \\
\text { GGTAGGACGGTTATGGTGTGTGATAGTAAGAGCAATC }\end{array}$ & 10 & 27.5 & 57.5 & 5 \\
\hline
\end{tabular}

Highlighted nucleotides represent forward (red) and reverse (blue) primer regions

Analyses of the N40 random region for each TCS R10 aptamers indicated an enrichment of $\mathrm{G}$ and $\mathrm{T}$ nucleotides across all sequences with $\mathrm{G}$ being the most abundant occupying over $40 \%$ of the $\mathrm{N} 40$ region. 
The evolutionary relationship between the identified aptamers was further investigated using phylogenetic analyses (Figure 4.4).

Figure 4.4: Phylogenetic relationships among TCS round 10 aptamers (H1 - H10)

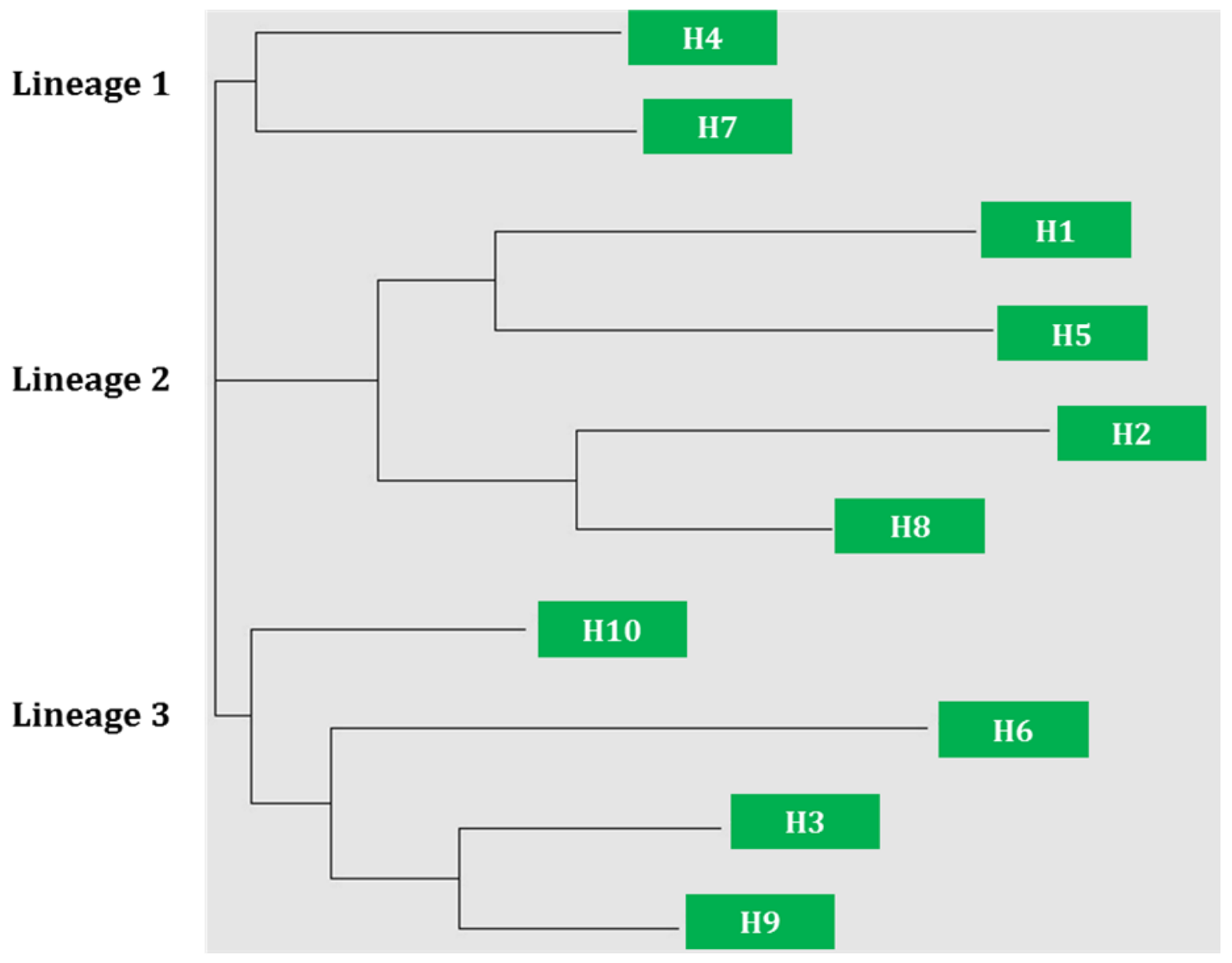

The phytogenic tree (Figure 4.4) indicated three major branches of evolutionary lineage. Aptamers H4 and H7 were grouped in lineage 1, which showed the closest relationship, and was also evident from their similar nucleotide compositions in the N40 random region (Table 4.4). Aptamers $\mathrm{H} 1, \mathrm{H} 5, \mathrm{H} 2$ and $\mathrm{H} 8$ were grouped in lineage 2, which when compared with lineage 1, appeared rather closely related each other although H8 was more distinct from the others. In lineage 3, aptamer H6 was more distinct compared with aptamers H3, H9 and H10, with H3 and H9 the most closely related. 
Nucleotides within the N40 region of each TCS R10 aptamer were then aligned to identify regions of homology (Figure 4.5) to assist in identifying shared conserved sequences.

Figure 4.5: Alignment of N40 regions for TCS round 10 aptamers (H1 - H10)

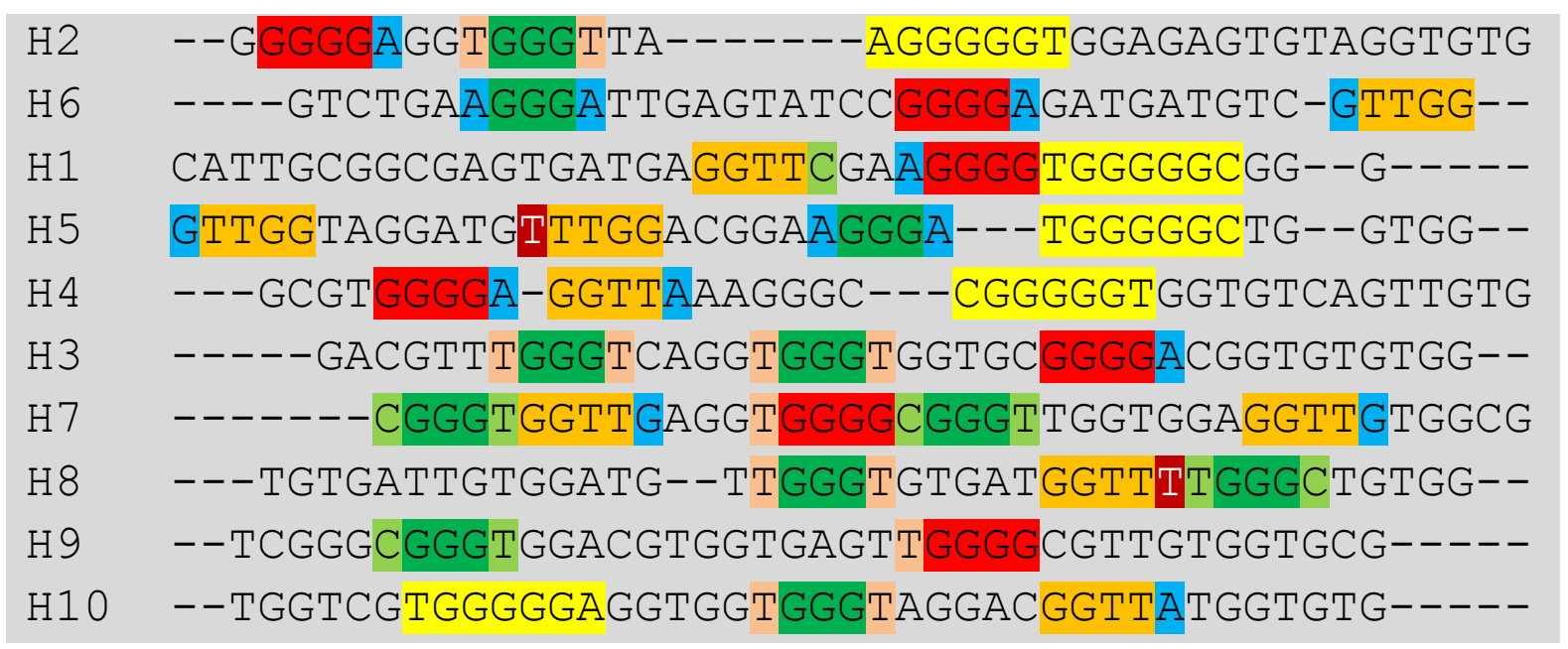

Highlighted nucleotides denoted conserved sequences.

Analysis of the N40 regions when aligned showed some conserved sequences such as: "XGGGX", where X can be both T, both A or C and T" (8 aptamers), "GGTTX", where X can be either A, T, C or G" (7 aptamers), "GGGGX” where X can be either A or T" (7 aptamers), and "TGGGGGX", where X can be either C or A" (5 aptamers) (Figure 4.5).

Secondary structures of TCS R10 aptamers were then predicted in order to find their folding characteristics between primer and random regions. Secondary structures of aptamers H2, H5 and H6 are shown in Figure 4.6, and the complete folding are shown in Appendix IV. 
Figure 4.6: Secondary structures of aptamers H2 (TR10H2), H6 (TR10H6) and H5 (TR10H5) demonstrating two, three and four stem-loop (SL) structures respectively

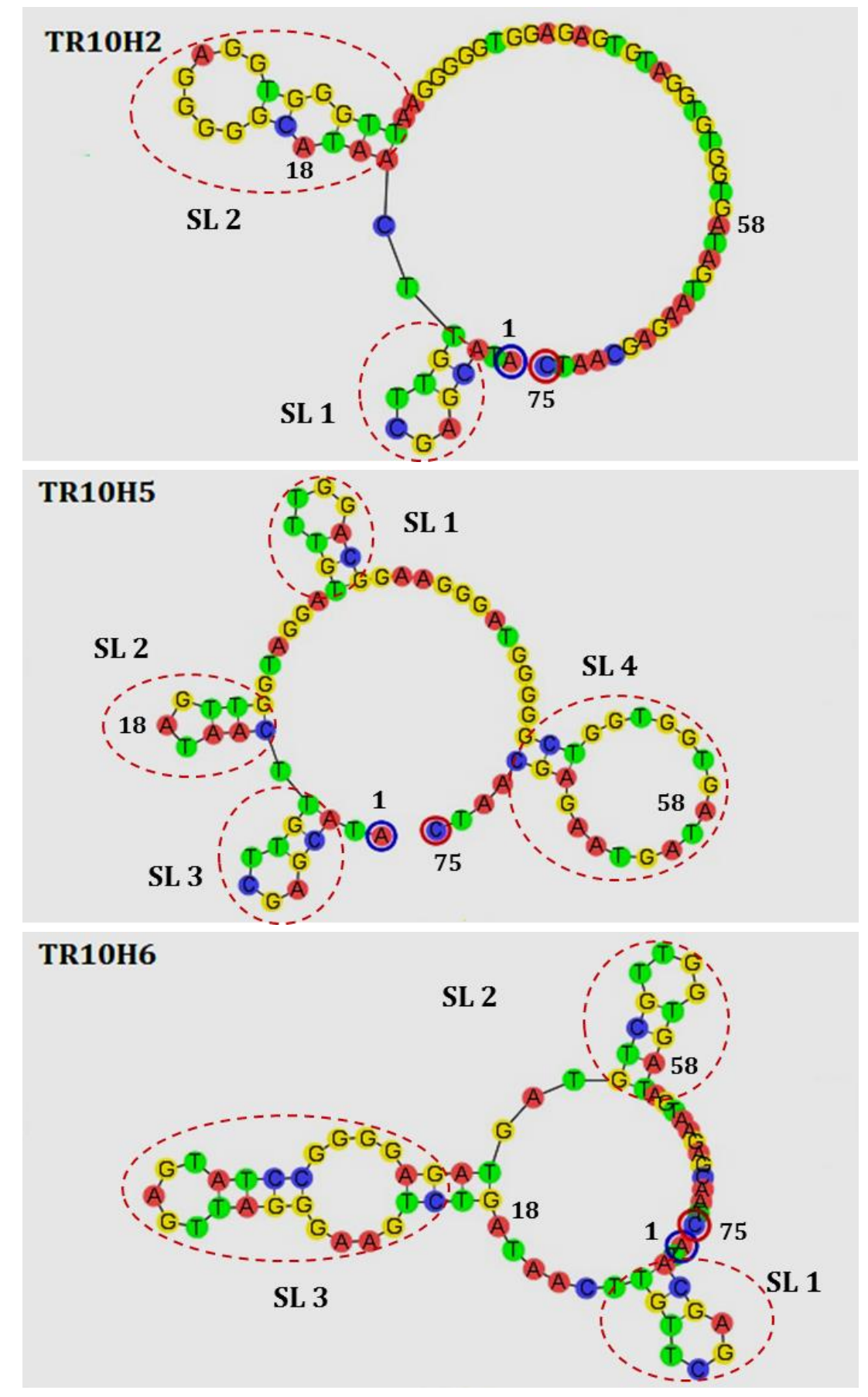

According to the folded-secondary structures (Figure 4.6 and Appendix IV), two variants (H7 and H9) were folded into similar linear hairpin loop-stem structures. Three variants, H2, H4 and H10 displayed two stem-loop structures. Another three variants H1, H6 and H8 displayed three stem-loop structures. Aptamer H5 displayed a four stem-loop structure. 


\subsubsection{Aptamer characterisation}

TCS R10 aptamers (H1 - H10) were initially characterised using the fluorescent imaging assay, and three aptamers H2, H5 and H6 were chosen to determine their $K_{d}$ values using both the fluorescent imaging and binding assays. Thereafter, the chosen aptamers were subjected to CD spectroscopy for assessing their levels of conformational change upon target bininding. Furthermore, the binding specificity of aptamer H6 was determined using the competitive binding assay.

\subsubsection{Binding affinity}

TCS R10 aptamers and the random sequence were screened individually for their affinity towards both control and TCS D matrices (Figure 4.7a).

As shown in Figure 4.7a, no visual fluorescent signals were observed from control D matrix for all sequences tested. The random sequence along with aptamers H7, H8, and H9 showed very little affinity towards TCS D matrix, whereas aptamers H1, H3, H4 and H10 all showed some affinity. In contrast, aptamers H2, H5 and H6 showed relatively high affinity with $\mathrm{H} 6$ being the highest amongst all aptamers tested. 
Figure 4.7a: Fluorescent images showing affinities of the random (Rand) sequence and TCS round 10 (R10) aptamers (H1 - H10) to control and TCS D matrices

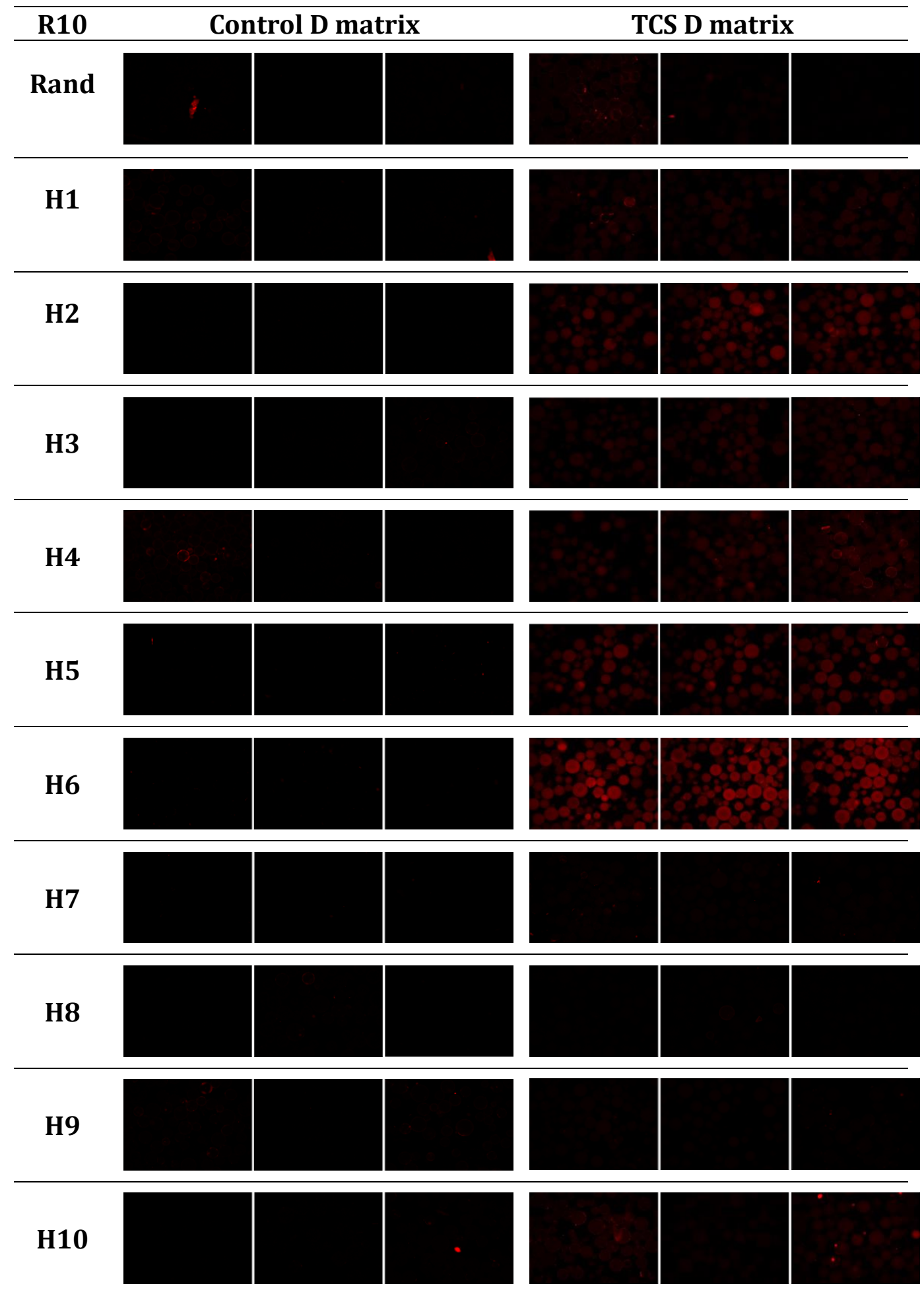

Images were captured under the same conditions via three independent experiments. 
The fluorescence intensity of each oligonucleotide-bound matrix was analysed to quantify TCS binding capabilities of TCS R10 aptamers (Figure 4.7b).

Figure 4.7b: Fluorescent counts representing binding affinities of the random (Rand) sequence and TCS round 10 (R10) aptamers to control and TCS D matrices

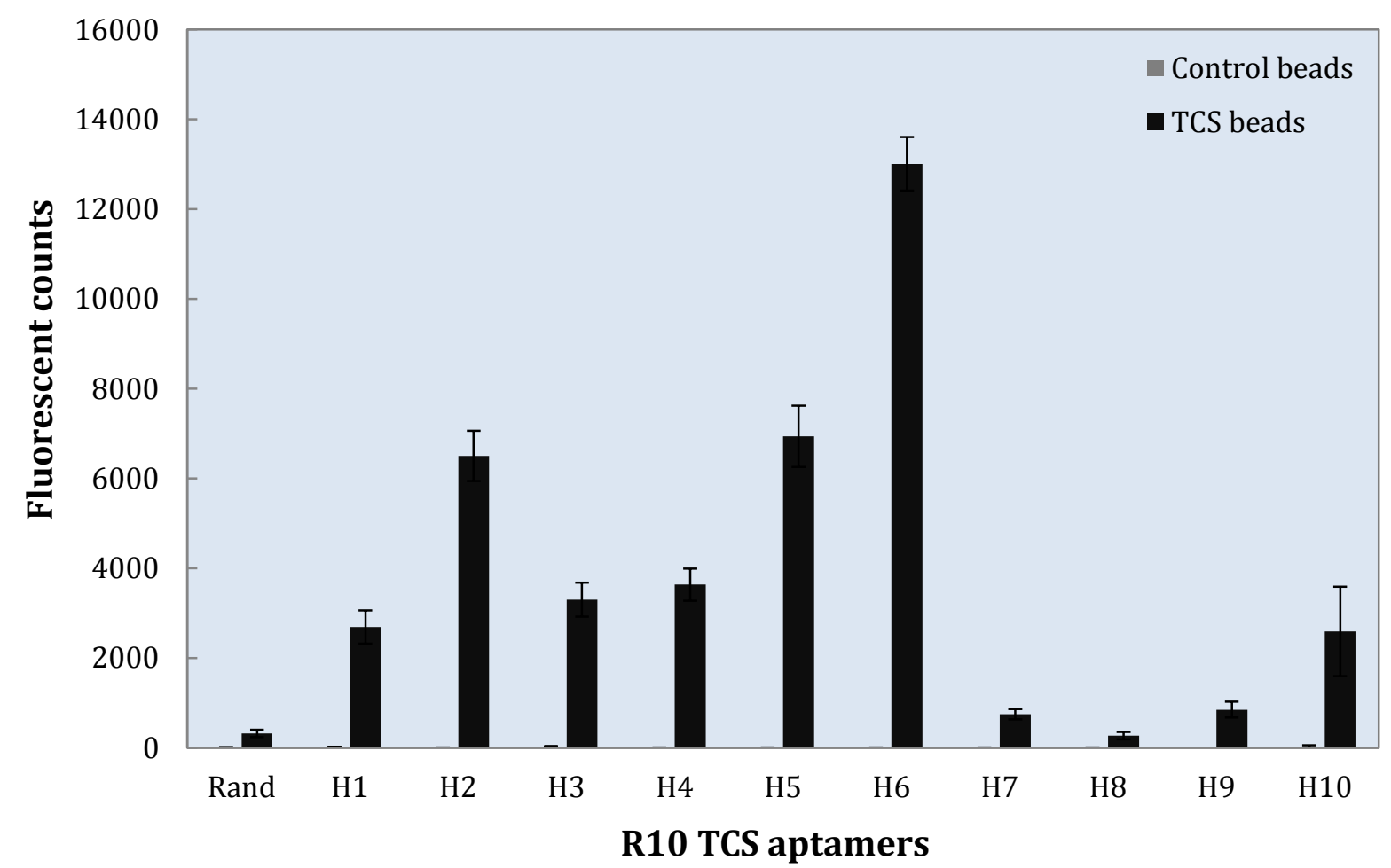

The error bars represent the standard deviation of the fluorescent counts measured via three independent experiments.

Using the fluorescent image analysis methodology, no fluorescent signals observed from control D matrix, and TCS aptamers H2, H5 and H6 showed highest affinity to TCS D matrix when compared with the remaining sequences being tested (Figure 4.7b). Therefore, these aptamers were chosen for $K_{d}$ determination.

\subsubsection{2 $K_{d}$ determination}

The $K_{d}$ values of aptamers $\mathrm{H} 2, \mathrm{H} 5$, and $\mathrm{H} 6$ were determined using both the fluorescent imaging and binding assays to establish the utility of the imaging method for $K d$ determination. 
In the fluorescent imaging assay, the determination of a $K d$ value for each sequence was repeated five times. Figure 4.8a summarises fluorescent images from one of the five experiments for each sequence and the complete images are shown in Appendix V.

Figure 4.8a: Fluorescent images of HEX-labelled aptamers (H2, H5 and H6) and the random sequence (Rand) prepared at $20-750 \mathrm{nM}$, binding to control and TCS D matrices

\begin{tabular}{|c|c|c|c|c|c|c|c|}
\hline $\mathbf{n M}$ & 750 & 750 & 550 & 350 & 150 & 50 & 20 \\
\hline H2 & & & & & & & \\
\hline
\end{tabular}
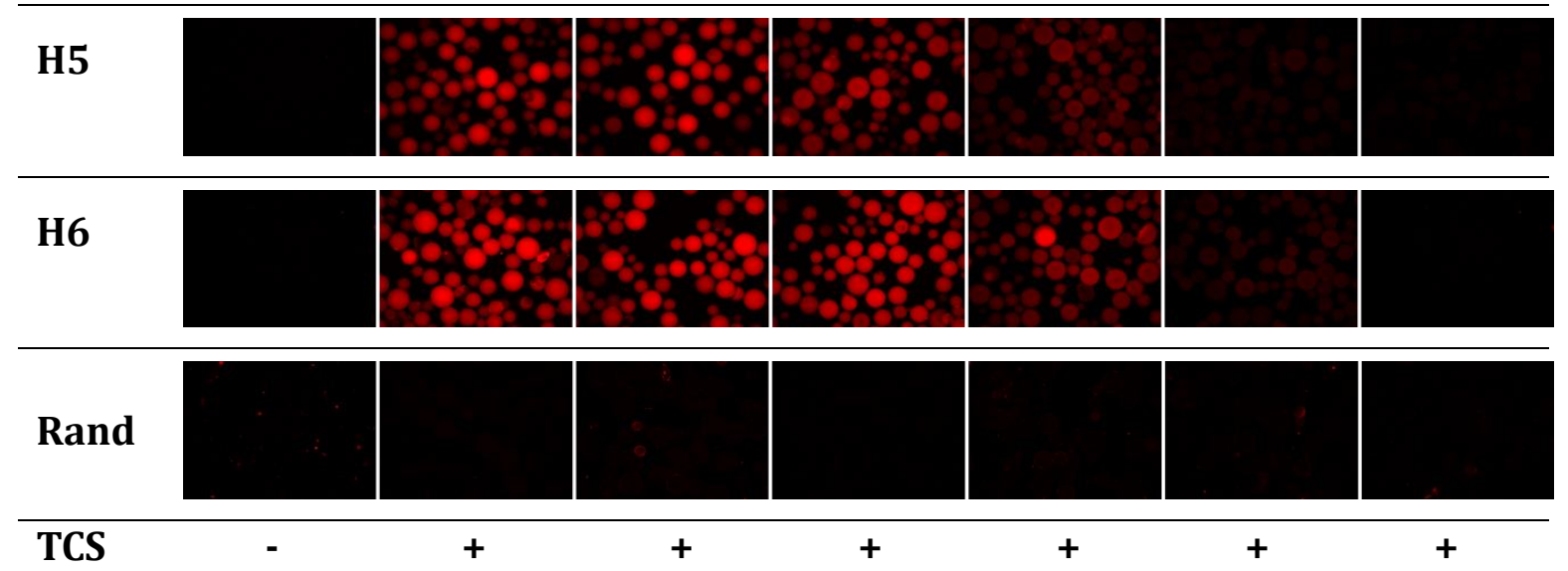

TCS (-) = control D matrix; TCS (+) = TCS D matrix and; each image was captured under the same conditions with an exposure time of 1 sec.

From visual examination (Figure 4.8a), the random sequence showed no affinity towards TCS D matrix even at the highest concentration of $750 \mathrm{nM}$. In contrast, aptamers H2, H5 and H6 showed binding to the TCS D matrix at $150 \mathrm{nM}$, and thereafter the binding increased progressively with increasing concentrations of aptamers. Furthermore, no fluorescent signals were observed by any sequences to the control D matrix. Thus, the negative image from the highest concentration only is shown in Figure 4.8a.

Thereafter, image analysis was performed to determine fluorescent intensity (Appendix VI). Based on image analysis, binding saturation curves for H2, H5, H6 and control (random) aptamers were generated to determine their $K_{d}$ values (Figure $4.8 \mathrm{~b}$ ). 
Figure 4.8b: Saturation curves of TCS aptamers H6 (i), H5 (ii), H2 (iii) and the random sequence (Rand) (iv) to TCS D matrix determined using the fluorescent imaging assay

i.

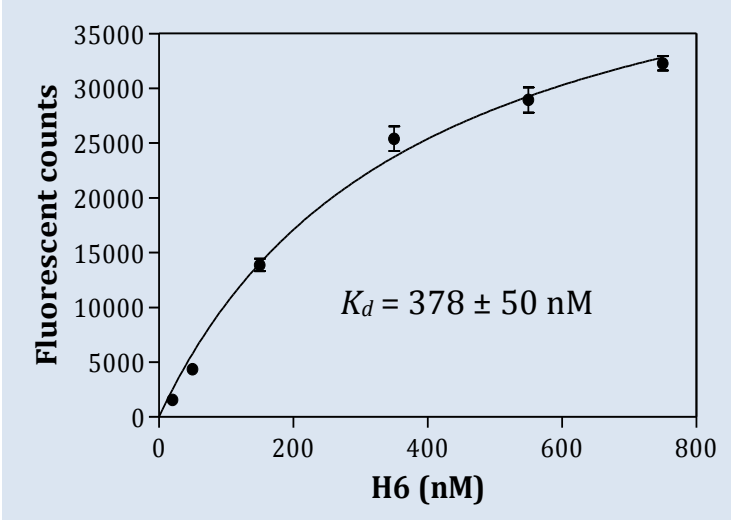

iii.

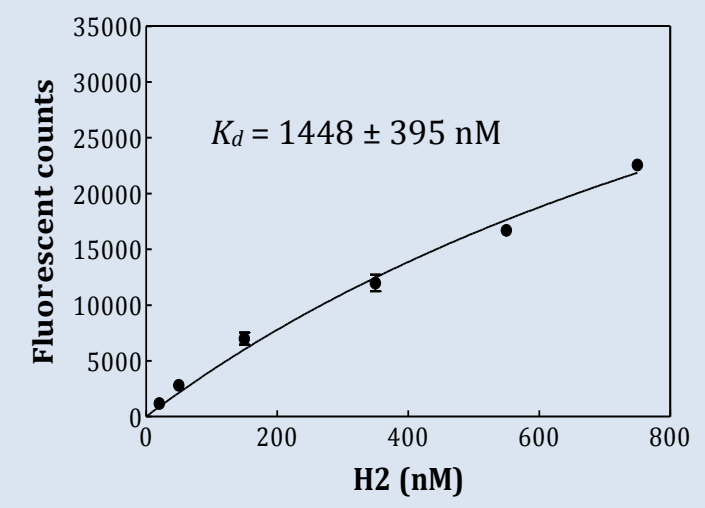

ii.

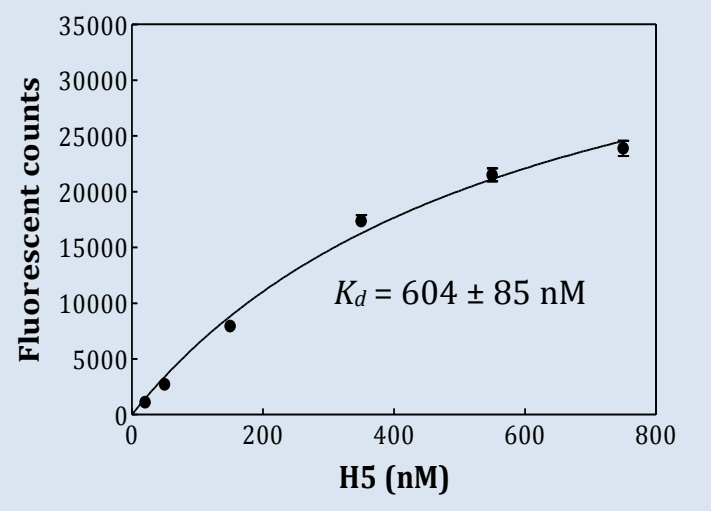

iv.

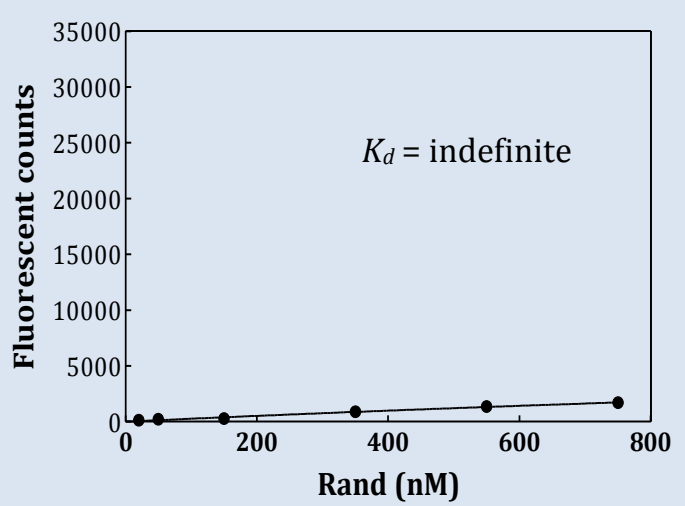

The error bars represent the standard deviation of the fluorescent counts measured via five independent experiments.

The mean \pm SD $K d$ values for aptamers $\mathrm{H} 6$ and $\mathrm{H} 5$ were determined to be $378 \pm 50$ and $604 \pm 85 \mathrm{nM}$ respectively (Figure 4.8b i and ii). Binding of TCS ligand to increasing amount of aptamer $\mathrm{H} 2$ did not reach saturation up to $750 \mathrm{nM}$ (Figure $4.8 \mathrm{~b}$ iii). This result suggests that higher concentration of the aptamer was required to reach saturation. Since the other two aptamers demonstrated better affinity than $\mathrm{H} 2$, no further increases in aptamer concentration were carried out for $\mathrm{H} 2$. However, the $K_{d}$ value for $\mathrm{H} 2$ was estimated by the Prism software to be $1448 \pm 395 \mathrm{nM}$. The random sequence (Figure $4.8 \mathrm{~b}$ iv) showed no significant binding to the TCS D matrix over all concentrations tested.

Aptamers H2, $\mathrm{H} 5$ and $\mathrm{H} 6$ as well as the random sequence were also assessed for their $K_{d}$ values (Figure 4.8c) using the fluorescent binding assay. 
Figure 4.8c: Saturation curves of TCS aptamers H6 (i), H5 (ii), H2 (iii) and the random sequence (Rand) (iv) to TCS D matrix determined using the fluorescent binding assay

i.

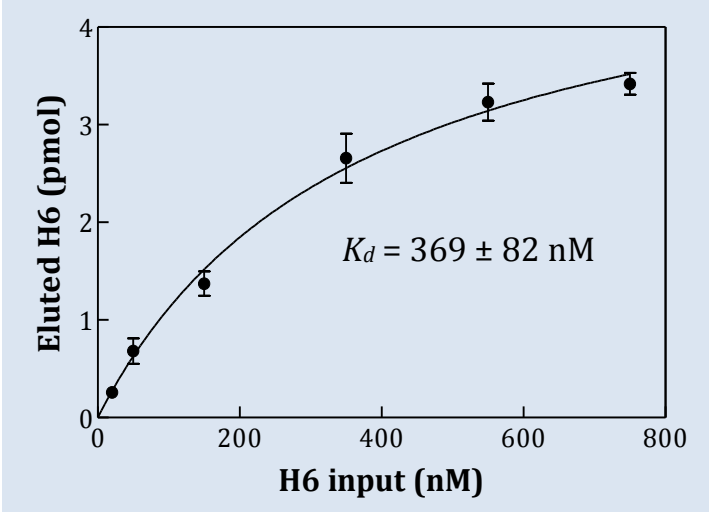

iii.

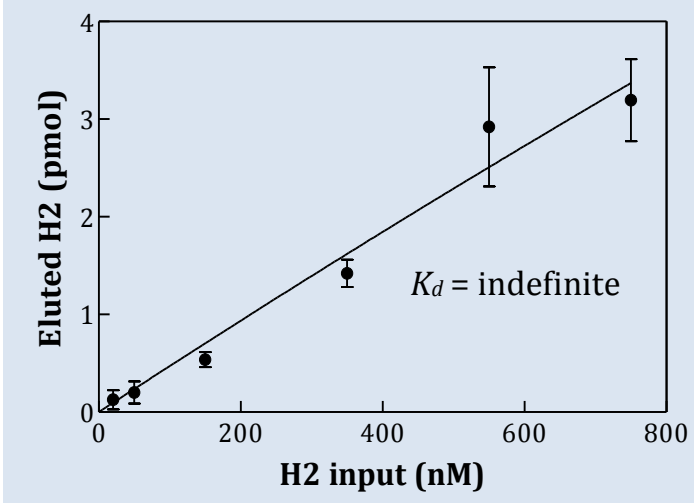

ii.

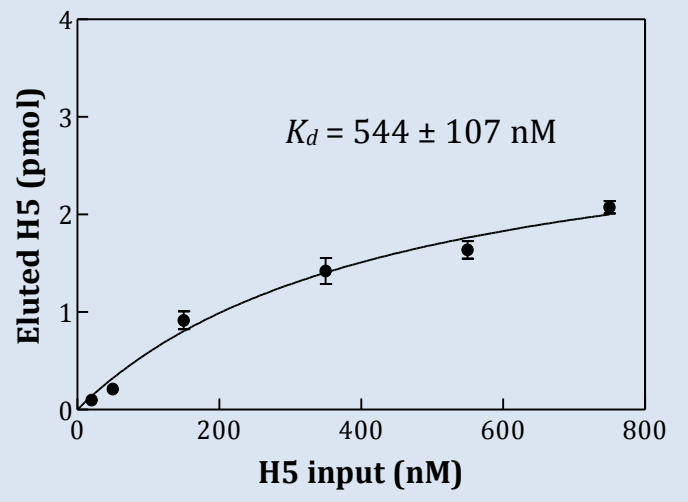

iv.

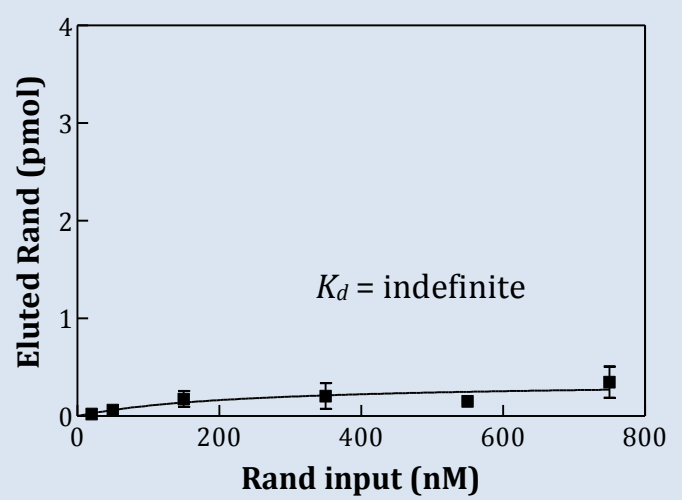

The error bars represent the standard deviation of the eluted aptamers measured via five independent experiments.

The $K_{d}$ value of H6 (369 $\pm 82 \mathrm{nM}$ ) determined by the affinity method (Figure 4.8c i) was similar to that determined by the imaging method (378 $\pm 50 \mathrm{nM}$ ), and the $K_{d}$ value of H5 (544 $\pm 107 \mathrm{nM}$ ) (Figure 4.8c ii) was also comparable to that found for the imaging assay (604 \pm 85$)$. No evidence that saturation binding was achieved with H2 (Figure 4.8c iii), similar to that found for the imaging method. Moreover, only a very low level of binding was evident with the random sequence (Figure $4.8 \mathrm{c}$ iv).

\subsubsection{Conformational change}

CD spectroscopy was used to investigate the in-solution binding activity of aptamers $\mathrm{H} 2$, H5 and H6 to TCS. CD spectra of these aptamers as well as the random sequence were recorded before and after binding to TCS for 60 mins (Figure 4.9 A to F). 
Figure 4.9: CD spectra (A to F) of $1 \mu \mathrm{M}$ TCS aptamers (H2, H5, and H6) and the random sequence (Rand), and also the independent detection of TCS at 2, 5 and $10 \mu \mathrm{M}$

A.

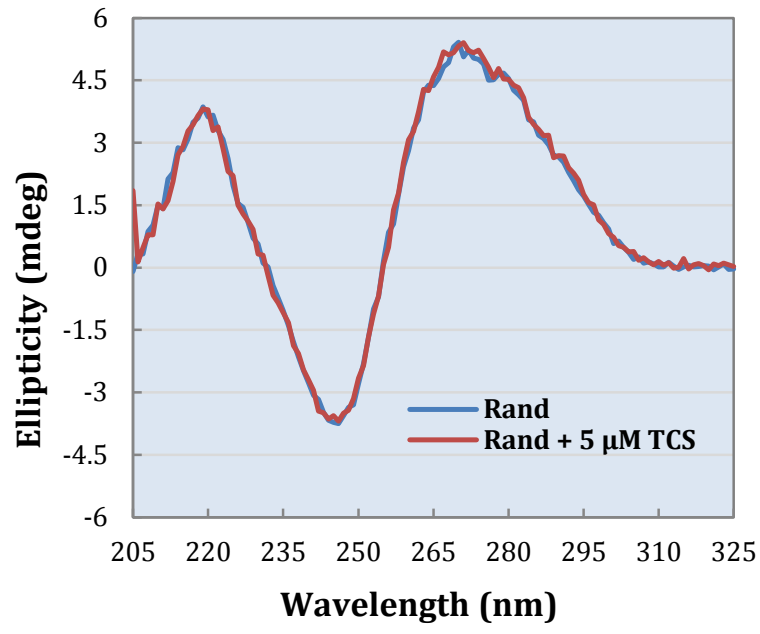

C.

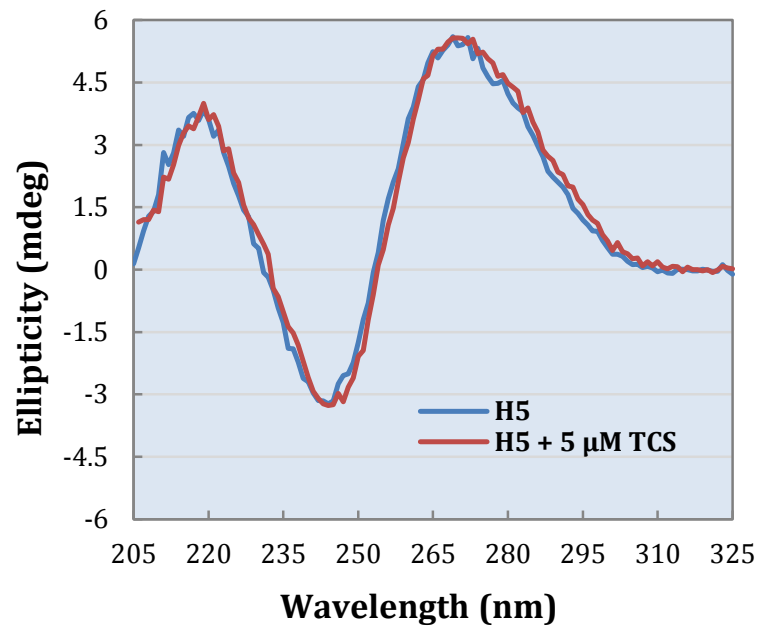

E.

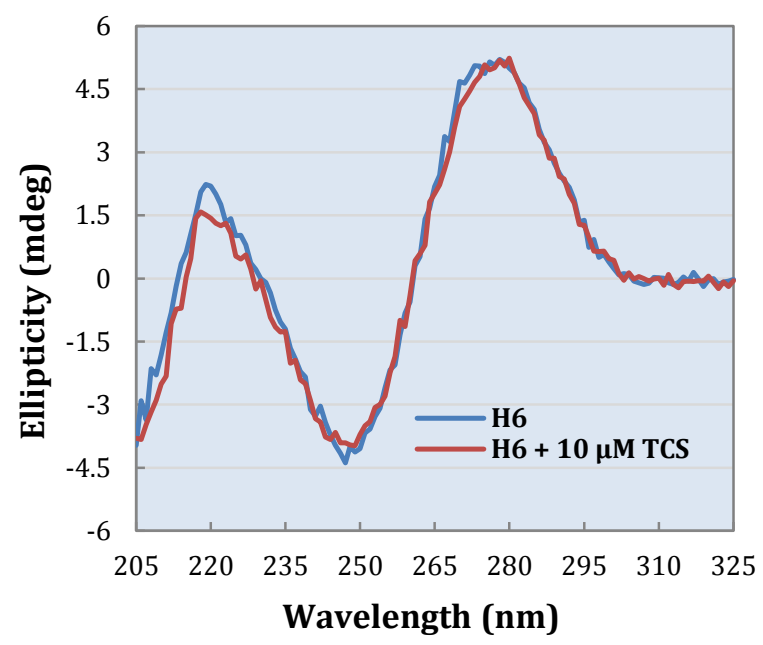

B.

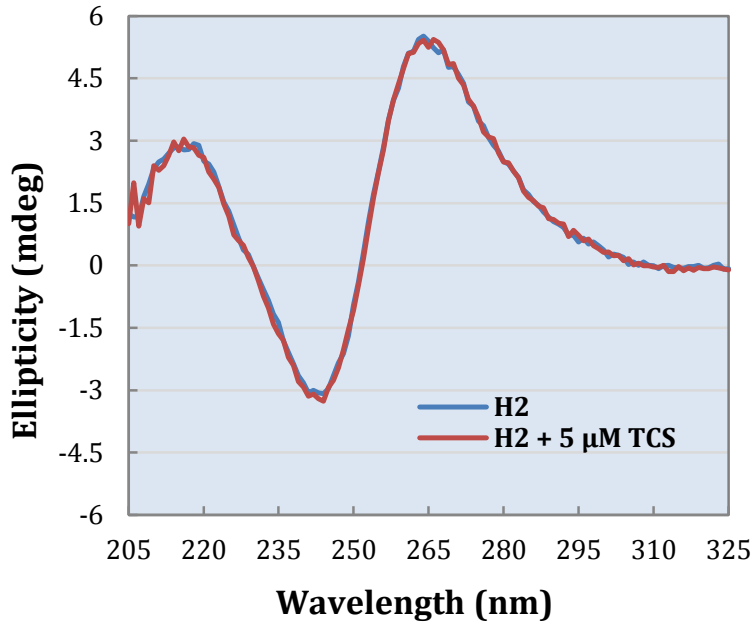

D.

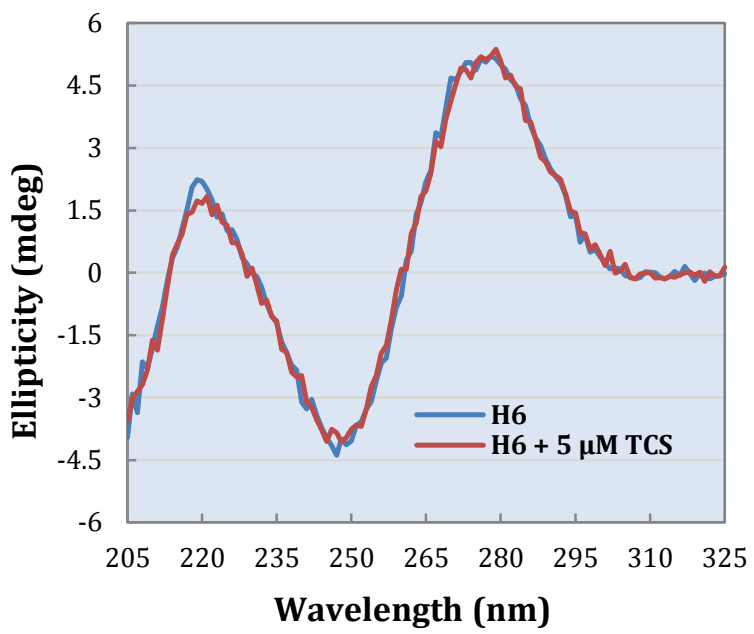

F.

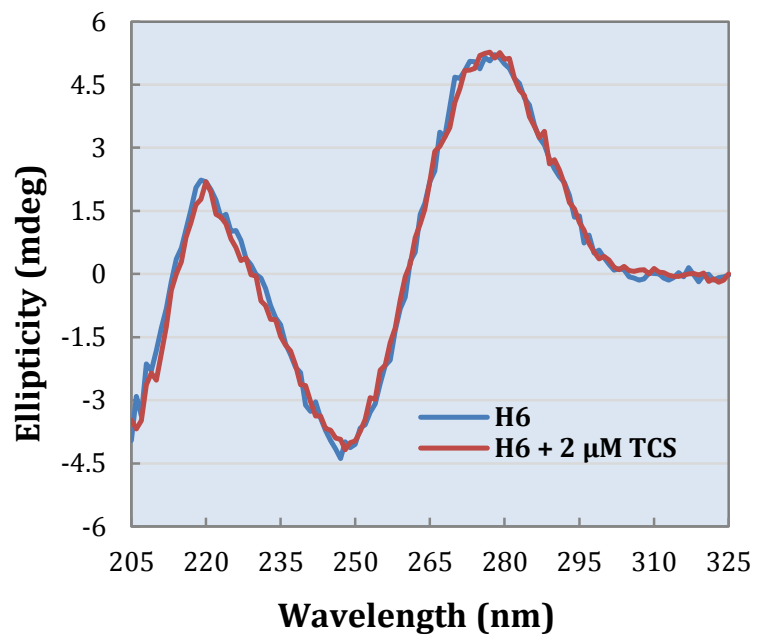


CD spectra of the random sequence and aptamer $\mathrm{H} 2$ overlapped each other before and after the present of TCS at $5 \mu \mathrm{M}$ (Figure $4.9 \mathrm{~A}$ and B), indicating no conformational change possessed by the sequences upon interaction with TCS. There was a very small shift to the right in the CD spectra of aptamer H5 after binding with TCS (Figure $4.9 \mathrm{C}$ ), but no overall changes in the ellipticity. Aptamer H6 showed a very small reduction of the ellipticity at 220 and $244 \mathrm{~nm}$ upon binding to $5 \mu \mathrm{M}$ TCS (Figure $4.9 \mathrm{D}$ ), and it appeared that the reduction increased in the presence of $10 \mu \mathrm{M}$ TCS (Figure $4.9 \mathrm{E}$ ), but decreased at $2 \mu \mathrm{M}$ TCS (Figure $4.9 \mathrm{~F}$ ). However, it cannot be conclusively determined whether the small changes was due to the aptamer associating with TCS or due to the increase of background noise observed in the CD spectra.

\subsubsection{Binding specificity}

Binding specificity of TCS aptamer H6 was investigated using the in-solution competitive binding assay. If the aptamer has better affinity towards competitive reagents (i.e. TCS, MTS, BPA and OE2), the aptamer will dissociate from the aptamer-bound matrix, thereby causing a reduction in the fluorescent signal (Figure 4.10a).

Figure 4.10a: Fluorescent images showing reduced fluorescent intensity of the H6 aptamer-bound matrix at the presence of competitive reagents in solution

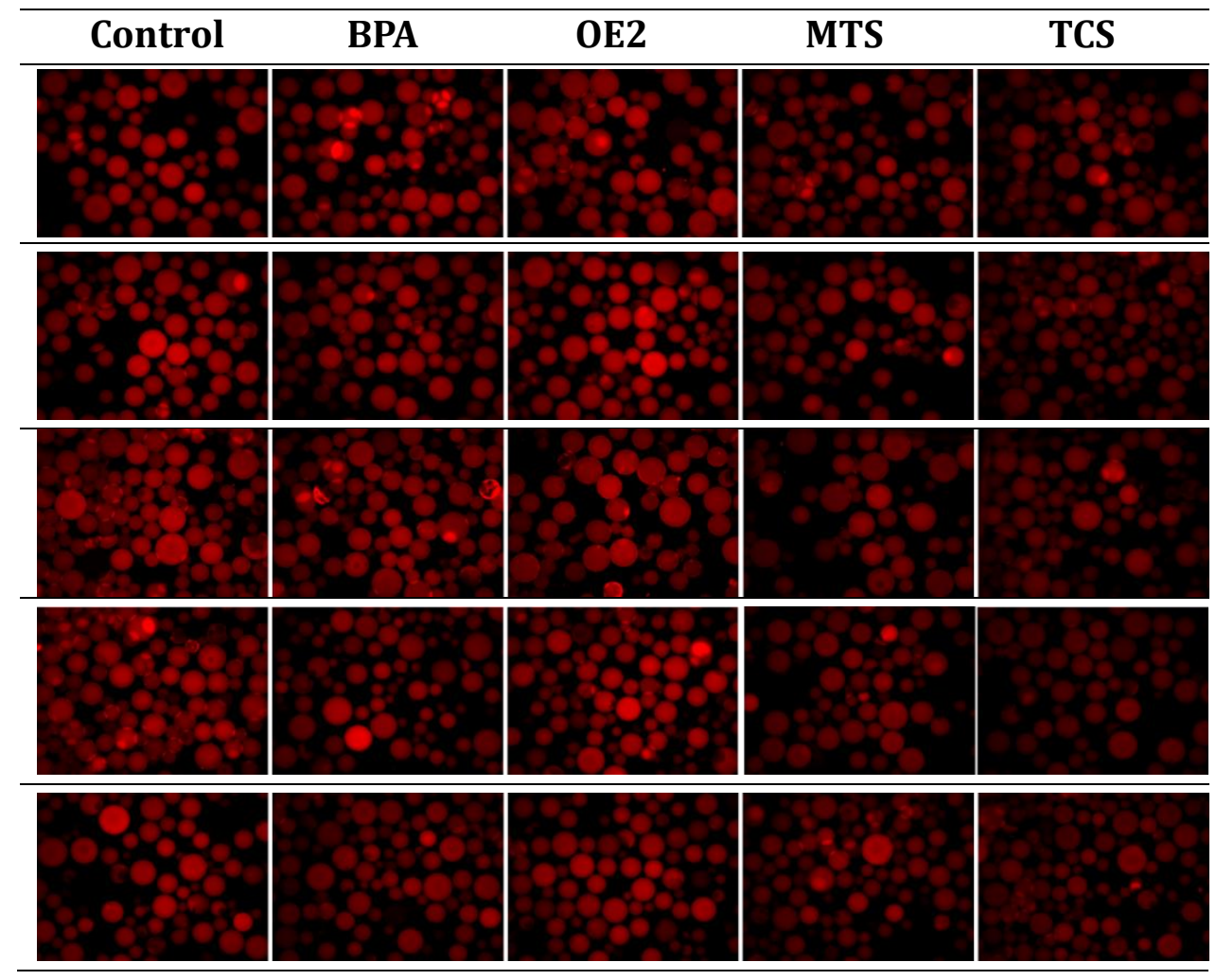

$\mathrm{BPA}=$ bisphenol A, OE2 = oestradiol, MTS = methyl triclosan and TCS $=$ triclosan. 
Visually, the competitive assay showed alterations to the fluorescence intensity of the aptamer-bound TCS D matrix following the introduction of MTS and TCS compared to the control (Figure 4.10a).

The fluorescence intensity was further evaluated using image analysis methodology (Figure 4.10b).

Figure 4.10b: Binding specificity of the H6 aptamer as assessed in competitive binding studies

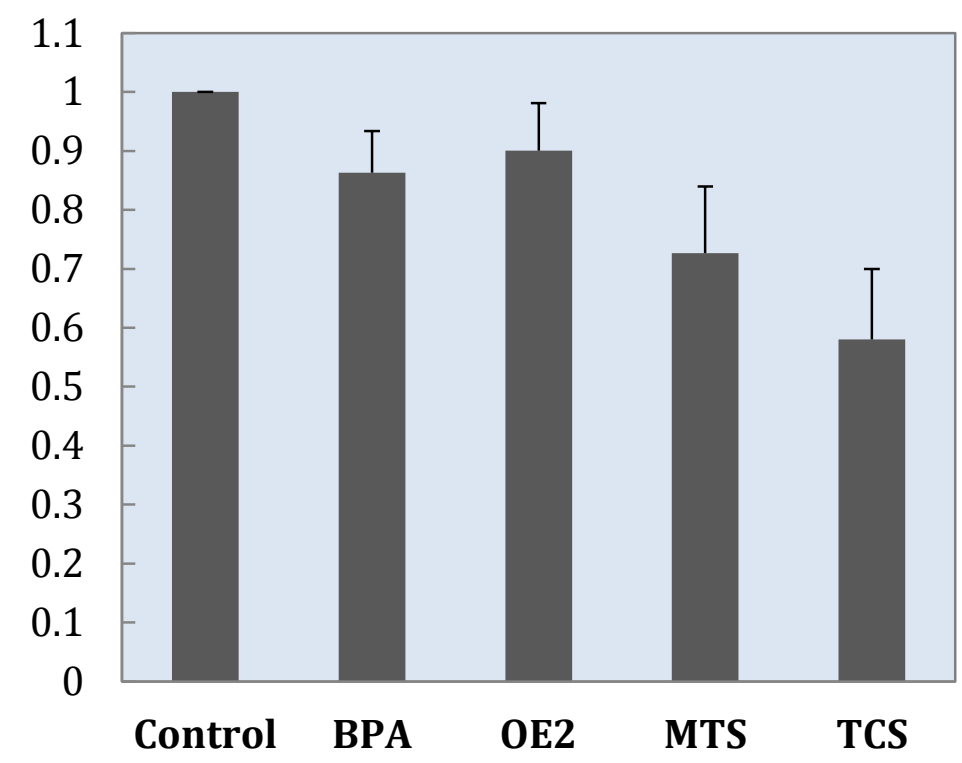

The error bars represent the standard deviation of the fluorescence reduction from 5 independent experiments with the sample reading normalised to the control values; BPA = bisphenol A, OE2 = oestradiol, MTS = methyl triclosan and TCS = triclosan.

TCS is the most specific target for aptamer H6 with over $40 \%$ reduction in the fluorescent signal (Figure $4.10 \mathrm{~b}$ ). The results also show that the aptamer has a binding affinity to MTS with around $25 \%$ fluorescent reduction, wherease the aptamer showed little binding to either BPA or OE2 as there was only $\sim 10 \%$ reduction observed for these molecules (Figure 4.10b). 


\subsubsection{Aptamer truncation study}

The $K_{d}$ values of the truncated aptamers were determined using the fluorescent imaging assay and by replicating the experiments five times. Figure $4.11 \mathrm{a}$ summarises fluorescent images for each aptamer from one of the five experiments and complete images are shown in Appendix VII.

Figure 4.11a: Fluorescent images of HEX-labelled aptamers 57H6F, 58H6R and 40H6B prepared at 20-750 $\mathrm{nM}$, binding to control and TCS D matrices

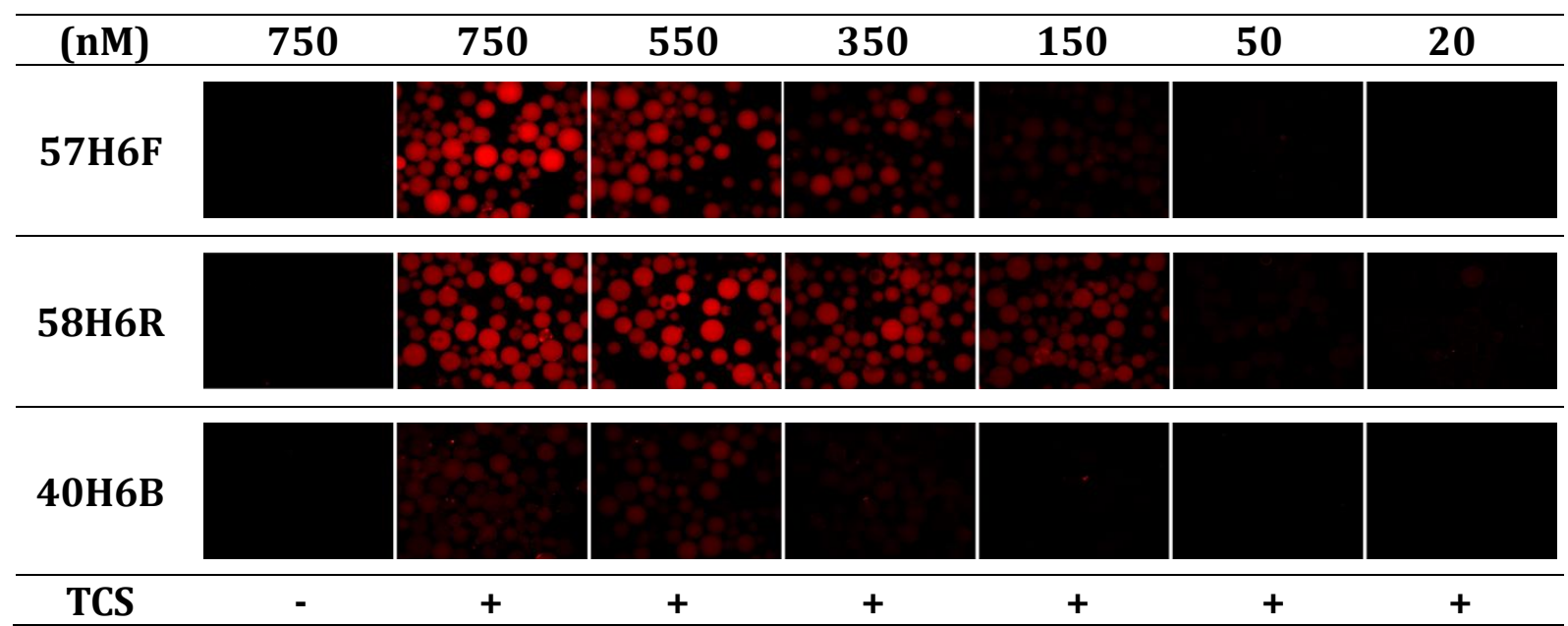

TCS $(-)=$ control D matrix; TCS $(+)=$ TCS D matrix and; each image was captured under the same conditions with an exposure time of $1 \mathrm{sec}$.

From visual examination (Figure 4.11a), the 40H6B had lost its affinity to TCS matrix even at the highest concentration of $750 \mathrm{nM}$. In contrast, the 57H6F and 58H6R showed binding to the matrix at 300 and $150 \mathrm{nM}$, respectively. Thereafter, binding increased progressively with increasing concentrations of the aptamers. Furthermore, no fluorescent signals observed from any sequences tested to the control matrix at $750 \mathrm{nM}$. Image analysis was performed to generate binding saturation curves for the truncated sequences in order to determine their $K_{d}$ values (Figure $4.11 \mathrm{~b}$ ). 
Figure 4.11b: Saturation curves of the truncated sequences 57H6F, 58H6R and 40H6B binding to TCS D matrix determined using the fluorescent imaging assay

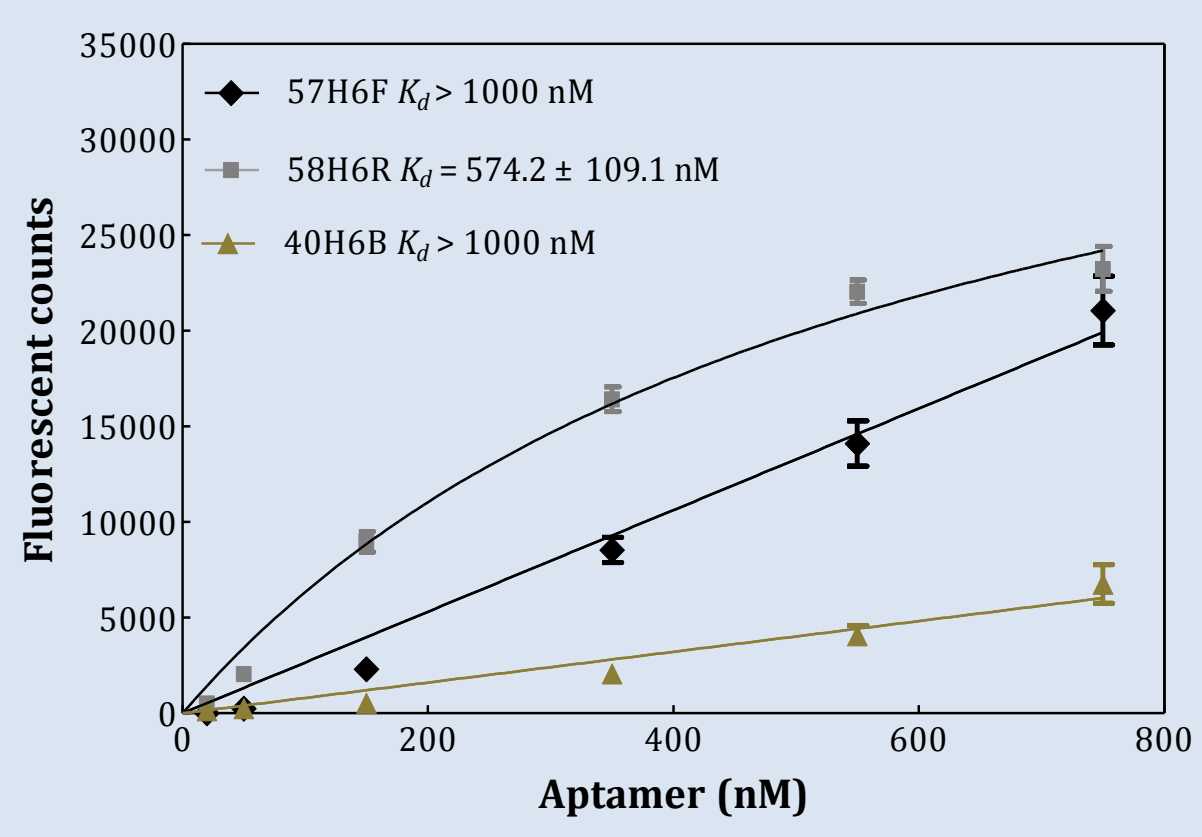

The error bars represent standard deviation of the fluorescent counts measured via five independent experiments.

As shown in Figure 4.11b, the binding of the truncated sequences $57 \mathrm{H} 6 \mathrm{~F}$ and $40 \mathrm{H} 6 \mathrm{~B}$ failed to reach saturation, whereas the $K_{d}$ value of $58 \mathrm{H} 6 \mathrm{R}(574 \pm 109 \mathrm{nM})$ is increased by almost two-fold than that for the full length sequence (378 $\pm 50 \mathrm{nM}$ ) (Figure 4.8b i).

\subsection{Discussion}

This chapter explored a revised-SELEX procedure for generating TCS specific ssDNA aptamers. The ten monoclonal TCS aptamers (named as H1 - H10) were generated after ten cycles of selection (Table 4.4). The aptamers showed improved binding affinity and specificity as a consequence of introducing modifications, such as the use of tRNA for matrix blocking, negative and counter selections, as well as real-time amplifications.

The fluorescent imaging assay proved to be suitable for aptamer characterisation. Utilising this method, three acceptable aptamers H2, H5 and H6 were identified, and their $K_{d}$ values were determined to be 1448, 603 and 378 nM (Figure 4.8b) respectively. 
Aptamer H6 showed an almost two-fold improvement in binding affinity with TCS compared with the best binding aptamer D5 $\left(K_{d}=700 \mathrm{nM}\right)$ from the basic-SELEX procedure. These three candidates H2, H5 and H6 were assessed for their in-solution binding activity using CD spectroscopy and competitive binding assay. These experiments suggested that aptamer H6 was the best candidate for binding to TCS. Although truncated versions of this aptamer showed reduced binding affinities to TCS, future investigation of $\mathrm{H} 6$ as a capture molecule is worthwhile.

In the revised-SELEX procedure, the use of tRNA formulated Blotto for matrix blocking proved to be effective in minimising non-specific adsorption caused by electrostatic interactions between oligonucleotides and the matrix. This is evidenced in the experiments, where the binding affinity of a 75 base-random sequence to both control and TCS D matrices was completely restricted in the presence of tRNA (Appendix VIII). The application of a $10 \mathrm{mg} / \mathrm{mL}$ tRNA Blotto formulation was standard practice in the revised-SELEX procedure. With this formulation, the random sequence and aptamers $\mathrm{H} 2$, H5 and H6 showed little non-specific adsorptions to control matrix even at the highest concentration of $750 \mathrm{nM}$ (Figure 4.8a).

Negative and counter selection strategies were applied in the revised-SELEX procedure to eliminate nucleotide sequences that bound to either the matrix or compounds sharing structural similarity to TCS. Typically, SELEX experiments perform a negative selection step using unconjugated matrix before the first round of selection (Silverman 2009). However, as individual sequences in the initial synthetic library are single copies, performing negative selection before the first round of SELEX can cause a significant reduction in the complexity (i.e composition) of the nucleotide pool. Therefore, in the revised-SELEX procedure used herein, a negative selection step with control D matrix was performed before the third round of SELEX, after the aptamer library had already been enriched twice. It was anticipated that this would ensure the presence of multiple copies for each putative aptamer sequence. To further enhance aptamer selectivity, counter selections with OE2- and BPA-matrices were also performed prior to selection rounds 6 and 8 respectively. OE2 and BPA have similar structures to TCS, thus removing aptamers that bind to these structures should increase the binding specificity for TCS. 
The performance of both negative and counter selection strategies were assessed during the progression of the selection procedure (Figure 4.3b). A gradual increase in aptamer affinity was noted for most positive selection rounds, except for the rounds 3 and 8 , where there was reduced fluorescent intensity compared with the previous rounds 2 and 7 respectively. This was not unexpected as negative selection and counter selection with control D and BPA-matrices were applied before these two rounds to remove non-specific sequences. Therefore, there was less aptamer bound to TCS D matrix and so that the matrices showed a reduced fluorescent signal.

In contrast, the use of OE2-matrix for counter selection before round 6 did not result in reduced fluorescent intensity. This indicated that only a few of the nucleotide sequences at the SELEX round 5 were binding with OE2. This is reasonable given the fact that the chemical structure of OE2 (a four ring-structure) is somewhat different from TCS (a two ring-structure) when compared with BPA (also a two ring-structure) (Figure 3.1, Chapter 3). After five rounds of positive selection, it was safe to assume that the selected polyclonal aptamer library contained sequences preferentially binding to the two-ring structure molecules, with very few sequences remaining that were specific to the four ringstructures such as OE2. The results are in agreement with the observation from the PCR amplification where only faint bands were observed when amplifying aptamer-bound OE2-matrix (Figure 4.2, lanes $9 \& 10$ ), whereas bands with reasonable intensity (Figure 4.2 , lanes $5 \& 6$ and $13 \& 14$ ) were observed with aptamer-bound control D and BPAmatrices indicating some sequences specific to these matrices have been removed from the polyclonal aptamer libraries. It is worth mentioning that counter selection using MTS was not performed in this study, as not only are the structural difference between MTS and TCS minimal, but also that MTS is a metabolite of TCS. It was considered that aptamers demonstrating affinity towards MTS would be also a useful indicator for the presence of TCS. The fluorescence imaging method showed that no further enrichment occurred during the last two rounds of selection (Figure 4.3b). Therefore, the selection was stopped at round 10, and the aptamer library from round 10 was cloned and sequenced to identify TCS specific monoclonal aptamers. 
Replacing conventional PCR amplification of an aptamer-bound matrix by real-time PCR amplification was a major improvement in the revised-SELEX procedure. Using real-time PCR, there were no signs of undesired PCR by-products formation after eight rounds of selection (Figure 4.2, lanes $11 \& 12$ ). Therefore, the revised-SELEX cycle proceeded beyond eight rounds. The only difference between performing a standard PCR and a realtime PCR is that the amplification process in the real-time procedure is stopped immediately after the real-time signal reaches a plateau, whereas a fixed 35 cycles of amplification was applied in the standard PCR process. This confirmed that over amplification of the aptamer-bound matrix was the cause for the formation of nonspecific PCR by-products.

Some potential applications of the fluorescent imaging assay were evaluated in therevised SELEX procedure. This method was used for monitoring the SELEX progression, characterising binding affinity and specificity of the selected aptamers, and determining their $K_{d}$ values. Specifically, the imaging method can be applied for cycle-based surveillance when monitoring the SELEX performance. In contrast, when using the dot blot assay in the basic-SELEX procedure, the first positive result was not evident until the sixth round of selection. And, beforehand it wasn't clear what was taking place during the SELEX procedure. Often, it is the initial rounds of SELEX which are critical in determining whether the conditions are appropriate for aptamer selection. Moreover, it was found that the imaging method required less effort when compared with dot blot assays, and the relatively simple visualisation of aptamer-bound matrices from each cycle provided useful information regarding the SELEX performance. When applying the imaging method for aptamer characterisation, the binding affinity of the R10 aptamers were ranked based on their fluorescent counts, and three promising aptamer candidates $\mathrm{H} 2$, $\mathrm{H} 5$ and H6, were identified in the initial affinity screening studies (Figure 4.7b). Later, the $K d$ values of these candidates were determined using both fluorescent imaging and binding methods. Results from both methods agreed each other (Figure 4.8b and 4.8c) confirming the novel fluorescent imaging assay is a suitable method for determining the $K_{d}$ values. 
In addition, the random sequence was included as a control in the aforementioned experiments to assess non-specific binding. This is a critically important step, as it confirms that the selected aptamer candidates are specific to TCS molecule instead of other moieties on the matrix surface. The resulting binding data that can be generated represent specific binding by aptamers, when the random sequence shows little or no affinity to TCS D matrix at all concentrations tested (Figure $4.8 \mathrm{~b}$ iv and $4.8 \mathrm{c}$ iv).

In-solution binding activities of the chosen aptamers H2, H5 and H6 were assessed using CD spectroscopy. As shown in Figure 4.9, aptamer H2 had no response in the CD spectrum at the presence of TCS. This is consistent with the finding that the H2 is a low affinity aptamer failing to reach saturation during the $K_{d}$ study. It is most likely that the affinity of H2 is matrix-based, just like the rhodamine case mentioned in the introduction to this chapter. The CD results for aptamer H5 were ambiguous, and the reason for the shift in its spectra (Figure $4.9 \mathrm{C}$ ) was unclear, whereas aptamer H6 showed a minimal change in the ellipticity in the CD spectra (Figure $4.9 \mathrm{D}, \mathrm{E}$ and F). Overall, the CD results are informative to conclude the binding activity of the chosen aptamers in solution. Therefore, the $\mathrm{H} 6$ aptamer was subjected to further characterisation using the competitive assay. In these studies, the H6 aptamer-bound matrix were subjected to an in-solution competition with TCS and structurally similar compounds (i.e. MTS, BPA and OE2). Dissociation of aptamer H6 from the matrix complex was observed when using TCS and MTS for competition as large fluorescent signal reductions observed (Figure 4.10a and 4.10b). In contrast, only small reductions (Figure 4.10a and 4.10b) observed with the use of BPA and OE2 for competition indicating that H6 is specific to TCS and also it's metabolite MTS. Although the competitive binding study has the potential to be developed as a sensing assay for TCS, the major drawback is that the sepharose beads are not an ideal carrier for such an assay. This is because the beads are not of uniform-size. Moreover, the synthesis of these beads leads to large batch to batch variation, and thus inconsistent assay performance. Therefore, it is reasonable to suggest that aptamers such as $\mathrm{H} 6$ will require a different assay platform for sensing applications. 
Truncation studies based on the use of aptamer H6 were carried out to identify TCS binding motifs. As evidenced from Figure 4.11a and 4.11b, the truncated versions aptamer H6 showed reduced affinity to TCS, and by removing both primer regions, the aptamer (i.e. 40H6B) lost its affinity to TCS completely. The message derived from these results is that just simply chopping off primer regions does not in itself help to identify the exact binding pocket for a selected aptamer, and further discussion on the aptamer truncation study will be addressed in Chapter 6. With respect to the information concerning nucleotide analyses, phylogeny and 2D structures of TCS R10 aptamers, discussion of these results in detail will be made in general discussion (see Chapter 6).

\subsection{Conclusion}

Selection modifications applied in the revised-SELEX procedure have led to the generation of TCS-specific aptamers with improved binding affinity and specificity. The best aptamer candidate H6 was fully characterised with respect to its $K_{d}$ value determination, in-solution conformational change and binding specificity, as well as through the truncation studies. These results suggest that this aptamer may well be an appropriate candidate for the development of an aptamer-based biosensor for detecting TCS. Finally, the fluorescent imaging assay was proved to be a superior technique for SELEX applications. 


\section{CHAPTER 5}

\section{DETECTION OF TRICLOSAN USING A GOLD NANOPARTICLE COLORIMETRIC METHOD}

\subsection{Introduction}

The use of gold nanoparticles (AuNPs) with ssDNA aptamers to measure binding interactions with small molecules is becoming an increasingly popular detection method. The chemical and optical properties of AuNPs make these nanoparticles an ideal candidate for use with ssDNA aptamers. In particular, AuNPs offer options of both chemical attachment of ssDNA molecules modified with reactive groups (e.g. -NH2 or $\mathrm{SH}$ ) and/or non-specific adsorption of ssDNA molecules onto their negatively-charged surface (Li and Rothberg 2004). In addition, the colour of a colloidal gold solution changes from red to violet during aggregation or from violet to red during disaggregation due to changes in inter-particle plasmon coupling and the resulting surface plasmon band shift (Elghanian et al. 1997). The change in the surface plasmon coupling of AuNPs allows for both qualitative (visual change in colour) and quantitative (UV-visible spectroscopy) measurements (Wang et al. 2007; Wei et al. 2007). Based on these properties, AuNPs have been utilised in the development of aptasensors by using the nanoparticles as the signal transducer with aptamers being the receptor. A number of AuNP-based aptasensors have been developed to detect either proteins or small molecules (Cheng et al. 2011; Li et al. 2011; Mei et al. 2013).

For example, by making use of chemical conjugation of oligonucleotides onto the gold surface and DNA hybridisation technology, Liu et al. designed ATP and cocaine aptasensors that use cross-linked AuNPs as the signal transducer (Liu and Lu 2006). In this system, ATP or cocaine aptamers is connected to a linker sequence. The AuNPs are functionalised with either a short oligo (oligo 1) whose sequence is complementary to the $5^{\prime}$ end of the linker sequence or another short oligo (oligo 2) whose sequence is complementary to the 3 ' end of the linker sequence and part of the aptamer sequence (Figure 5.1a). 
Figure 5.1a: An aptasensor utilising cross-linked gold nanoparticles

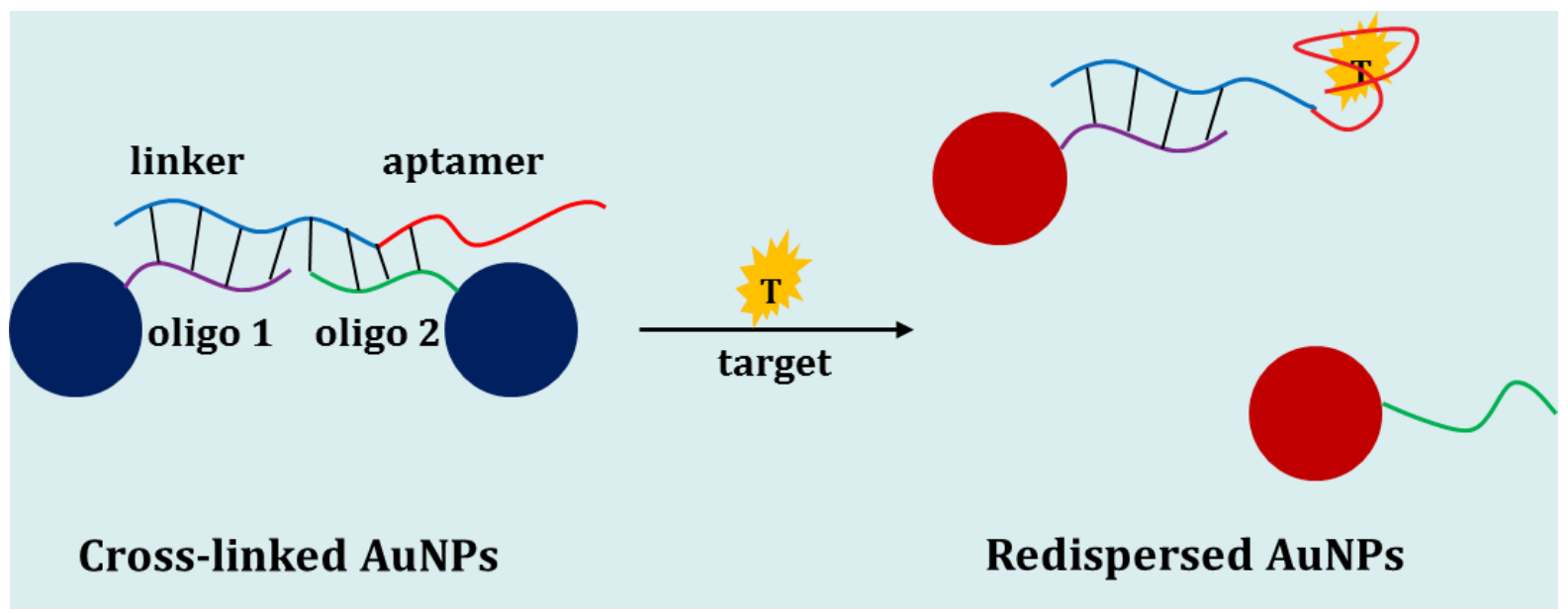

When the target is absent, the aptamer with the linker sequence hybridised to both oligo 1 and oligo 2, thereby crosslinking the AuNPs and forming aggregates that appear violet in solution. To accommodate the target molecule, the aptamer adopted a folded structure, thereby displacing the oligo 2 from the linking arm. This resulted in the breakdown of the aggregates and a violet-to-red colour change was observed in solution. This approach relies on a physical change of AuNPs from aggregation state to free dispersion in solution. This to some extent is less sensitive for target detection, since the AuNPs once aggregated are difficult to disaggregate presumably because of Van der Waals forces or steric hindrance restricting the accessibility of biomolecules into the aggregates (Zhao et al. 2007). As stated in this study at least $0.5 \mathrm{mM}$ ATP and $1 \mathrm{mM}$ cocaine are required to break down the AuNP aggregates and result in a noticeable colour change by naked-eye (Liu and Lu 2006).

Alternatively, Zhao et al. developed an aptasensor for ATP detection that utilises the same principle of oligonucleotide conjugation and DNA hybridisation, but relies on saltinduced aggregation of uncross-linked AuNPs (Zhao et al. 2007). The AuNPs used in this aptasensor are functionalised with a short oligonucleotide whose sequence is complementary to part of the aptamer sequence (Figure $5.1 \mathrm{~b}$ ). 
Figure 5.1b: An aptasensor utilising uncross-linked gold nanoparticles

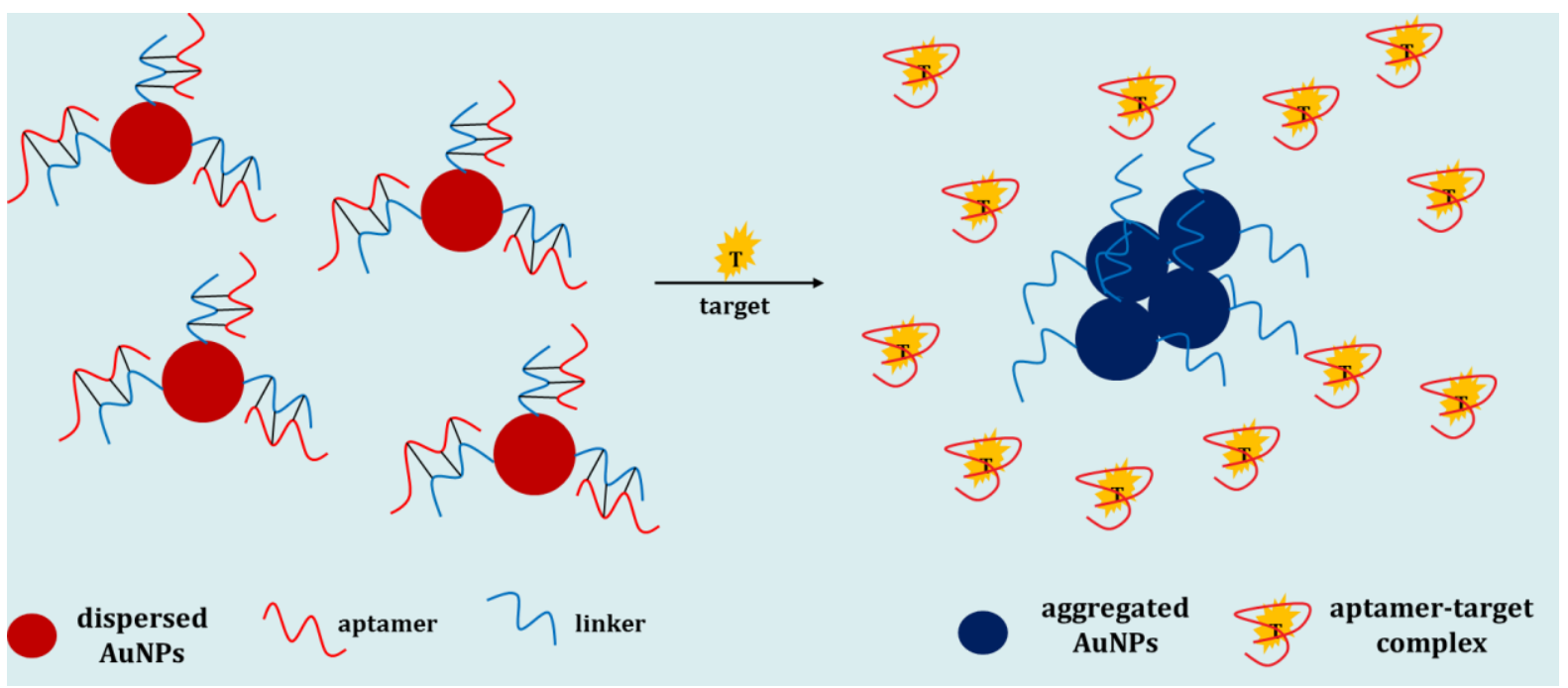

The aptamer sequence is hybridised to the short oligonucleotide in the absence of its target molecule, but quickly dissociate from the oligonucleotide when the target molecule is present. Since un-hybridised AuNPs are less stable at a specific salt concentration compared to their hybridised counterparts, the AuNPs aggregate and result in a red to violet colour change in solution. When applying the same ATP aptamer, the use of this uncross-linked AuNPs showed better sensitivity with a detection limit around $10 \mu \mathrm{M}$, which is approximately 20 times better than what was achieved with the cross-linked aptasensor (Figure 5.1a).

Both the above-mentioned studies involved a chemical conjugation of oligonucleotides to the surface of AuNPs. As mentioned earlier, ssDNA molecules can also electrostatically adsorb onto the surface of AuNPs, thereby stabilising the AuNPs against aggregation at certain salt concentrations ( $\mathrm{Li}$ and Rothberg 2004). The ability of anchoring ssDNA molecules onto the gold surface via non-specific adsorption has inspired many researchers to develop a new type of AuNP-based aptasensor referred to as the aptamerprotected AuNP aptasensor. Alsager et al. reported an ultrasensitive colorimetric detection of $17 \beta$-oestradiol (OE2) based on the use of an OE2 aptamer-protected AuNP aptasensor as demonstrated in Figure 5.1c (Alsager et al. 2015). 
Figure 5.1c: An aptasensor utilising aptamer-protected AuNPs

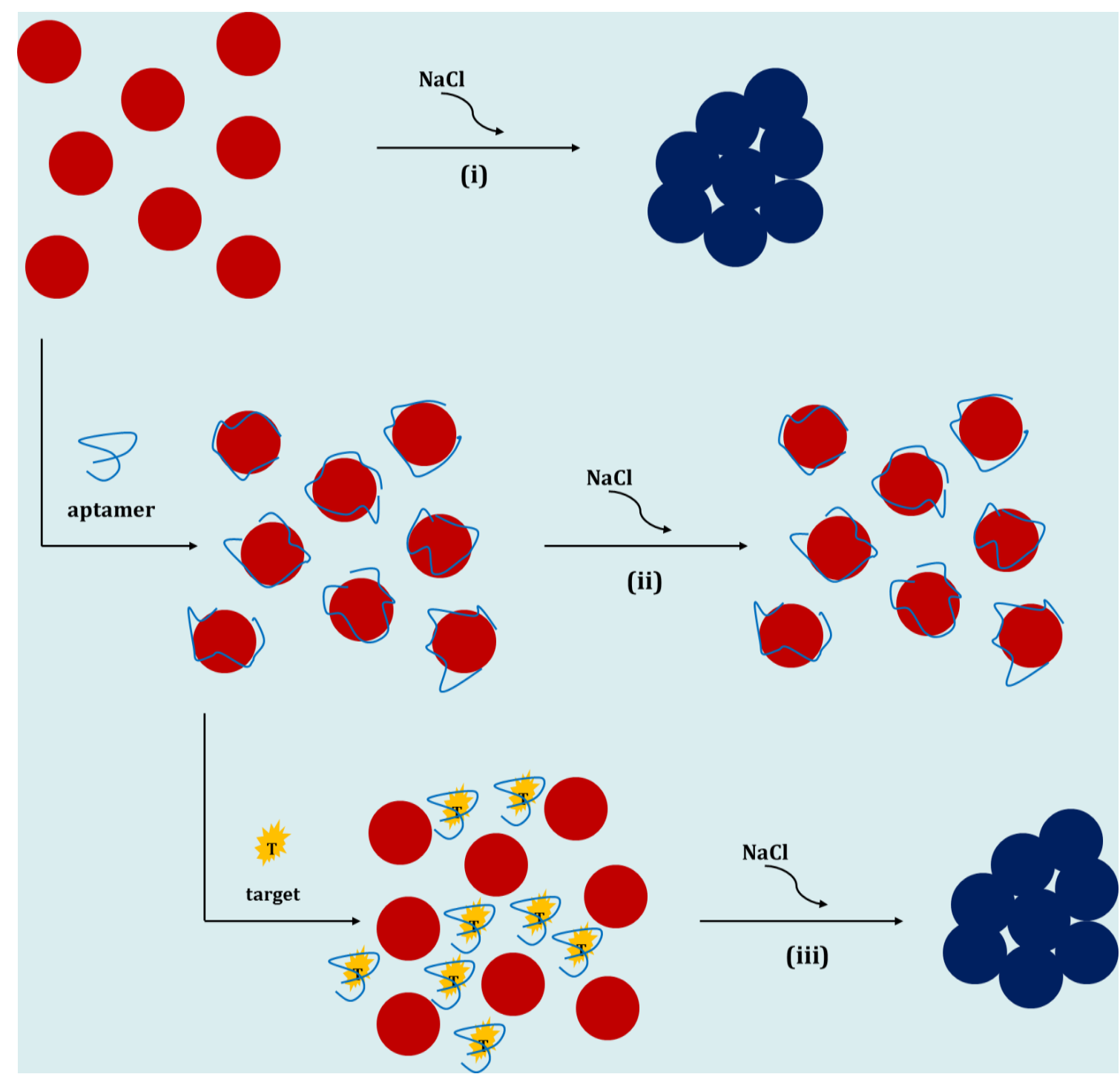

(i) monodispersed (red) AuNPs aggregating (blue) upon the addition of $\mathrm{NaCl}$; (ii) addition of an aptamer leads to the coating of the AuNPs, thereby preventing aggregation and; (iii) addition of the aptamer target molecule leads to the aptamer preferentially binding to the target resulting in the isolated AuNPs aggregating following the addition of $\mathrm{NaCl}$.

This aptasensor utilised non-specifically adsorbed OE2 aptamer on the surface of AuNPs dissociating upon the introduction of OE2. This was expected to occur as once OE2 was specifically bound to the aptamer, it would likely lead to a change in the aptamer conformation from a random flexible structure to a rigid tertiary structure. It was reasoned that an $\mathrm{OE} 2$ aptamer with a random flexible structure would be able to envelop and protect the AuNPs from $\mathrm{NaCl}$-induced aggregation, whereas with a rigid tertiary structure the aptamer could not protect the nanoparticles. 
Following the addition of $\mathrm{NaCl}$, the AuNPs in the presence of OE2 aptamer would remain dispersed, whereas in the presence of OE2, the aptamer would dissociate and AuNPs would aggregate. The OE2 aptasensor was able to detect OE2-spiked rat urine samples with a LOD of 200 pM (Alsager et al. 2015). Other examples of using aptamer-protected AuNPs for sensing include the detection of, oxytetracycline with a $L O D$ of $25 \mathrm{nM}$ (Kim et al. 2010), ochratoxin A with a LOD of $20 \mathrm{nM}$ (Cheng et al. 2011), and kanamycin with a LOD of $25 \mathrm{nM}$ (Song et al. 2011). Furthermore, a multiplex detection system enabling the measurements of sulfadimethoxine, kanamycin, and adenosine at the same time has been developed (Niu et al. 2014).

Overall, the aforementioned studies demonstrate that aptamer-protected AuNP aptasensors are suitable for the detection of small molecules, and the $L O D$ achieved in this approach is much lower than for those approaches using either uncross-linked or crosslinked AuNPs. Inspired by these studies, TCS aptamer H6 generated from the revisedSELEX procedure was tested for its utility in an aptamer-protected AuNP assay system (i.e. an aptasensor). Therefore, the aim of this chapter was to explore the development of an AuNP-based TCS aptasensor with the objectives of: [i] confirming the applicability of TCS aptamer H6 for the construction of an aptasensor; [ii] optimising the working conditions for the constructed aptasensor and; [iii] confirming the sensitivity and specificity of the aptasensor for TCS detection in-solution.

\subsection{Methods}

The work undertaken below using an AuNP-based system was developed with Drs Omar Alsagar and Shalen Kumar (Victoria University of Wellington, NZ). All materials, reagents and suppliers are shown in Appendix I.

\subsubsection{Synthesis and characterisation of AuNPs}

AuNPs used in the present study were chemically synthesised in the laboratory using the classical citrate reduction method (Grabar et al. 1995). Briefly, a $100 \mathrm{~mL}$ chloroauric acid solution ( $1 \mathrm{mM})$ was added to a conical flask and heated to boiling with constant stirring. Subsequently, a $10 \mathrm{~mL}$ sodium citrate solution $(38.8 \mathrm{mM})$ was added, and the resulting reaction mixture stirred for another 15 mins with constant heating. 
During this interval, the reaction mixture underwent colour changes from transparent to deep blue and finally to wine red indicating the successful formation of AuNPs. Thereafter, the synthesised AuNPs were visualised under a JEOL USA Transmission Electron Microscope (TEM) (JEOL Solutions for Innovation, Peabody, Massachusetts, USA), and a TEM image was captured at $20 \mathrm{~nm}$ resolution. Furthermore, an UV-visible absorption spectrum of the AuNPs was recorded from 200 to $800 \mathrm{~nm}$ using an Agilent $8453 \mathrm{UV}$ visible spectrophotometer (Agilent Technologies, Santa Clara, California, USA) with a 10 $\mathrm{mm}$ path length quarts cuvette at RT.

\subsubsection{Optimisation of AuNPs for biosensing}

Before constructing an AuNP biosensor, the AuNPs were investigated with respect to their salt tolerance, target effect and also the optimal amount of aptamer required for protection. The AuNPs used for these optimisation experiments were prewashed to remove excess amount of citrate ions. Specifically, the AuNPs (20 mL) were evenly distributed-into microcentrifuge tubes (each $1 \mathrm{~mL}$ ), and centrifuged at 12,500 x g for 30 mins at RT. Following centrifugation, the supernatant from each tube was discarded and the resulting pellets were combined and restored to the original volume of $20 \mathrm{~mL}$ using $\mathrm{dH}_{2} \mathrm{O}$.

\subsubsection{Salt tolerance}

$\mathrm{NaCl}$-induced aggregation of the unprotected AuNPs was undertaken to determine the optimal $\mathrm{NaCl}$ concentration needed to induce the aggregation. In a typical experiment, the prewashed-AuNPs were pipetted into 9 microcentrifuge tubes (each $100 \mu \mathrm{L}$ ). This was followed by the addition of $12 \mu \mathrm{L}$ of $\mathrm{dH}_{2} \mathrm{O}$ to each tube. Thereafter, a total of 9 different concentrations of $\mathrm{NaCl}$ solutions were added (8 $\mu \mathrm{L}$ per tube) to obtain final concentrations of $\mathrm{NaCl}$ ranging from 0 to $53.3 \mathrm{mM}$. The resulting solutions were then agitated using a wrist-flicking action for $30 \mathrm{sec}$ and then incubated for $15 \mathrm{mins}$ at RT. This step, referred to as the "wristflick-incubation", was used as a standard preparation step for the remaining work. Following the wristflick-incubation, samples were aligned on a lab bench, and a picture was then taken using a Nikon 200 digital camera to visually determine the optimal $\mathrm{NaCl}$ concentration (i.e. the minimal amount of $\mathrm{NaCl}$ used to fully induce the aggregation of the nanoparticles) by naked eye. This photograph and visualisation step was also a standard practice adopted for the following experiments. 


\subsubsection{Target effect}

The effect of TCS on the AuNPs was investigated to eliminate the possibility of TCSinduced aggregation. Briefly, the prewashed-AuNPs were pipetted into 9 microcentrifuge tubes (each $100 \mu \mathrm{L}$ ), followed by the addition of $8 \mu \mathrm{L}$ of $\mathrm{dH}_{2} \mathrm{O}$ to each tube. Thereafter, a total of 9 different concentrations of TCS solution were added ( $12 \mu \mathrm{L}$ per tube), so that the final concentration of TCS in each tube varied from 0 to $2000 \mathrm{nM}$. The resulting solutions then underwent the wristflick-incubation treatment. This was followed by the photograph and visualisation step to check for any formation of AuNP aggregates in each tube.

\subsubsection{Optimisation of aptamer concentration}

After finding the optimal $\mathrm{NaCl}$ concentration and excluding the possibility of target effect, the amount of aptamer required to protect AuNPs from aggregation was optimised. In brief, the prewashed-AuNPs were pipetted into 9 microcentrifuge tubes (each $100 \mu \mathrm{L}$ ). This was followed by the addition of $12 \mu \mathrm{L}$ of TCS H6 aptamer solutions each prepared in $\mathrm{dH}_{2} \mathrm{O}$ and containing different amount of the aptamer (ranging from 0 to $0.5 \mathrm{pmol}$ ). The resulting mixtures were incubated at RT for 60 mins to allow for the formation of aptamer-protected AuNPs. Following incubation, $8 \mu \mathrm{L}$ of $\mathrm{NaCl}$ solution prepared at the optimal concentration was added to each aptamer-protected AuNP solution. The final concentration of the aptamer used for protection varied from 0 to $140 \mathrm{nM}$. Thereafter, the resulting solutions were subjected to the wristflick-incubation treatment, photography, and the visualisation step to determine the optimal aptamer concentration required for protection. The concentration, determined as the minimal amount of an aptamer to fully prevent the $\mathrm{NaCl}$-induced AuNP aggregation, was chosen to construct the aptasensor for TCS detection as described next.

\subsubsection{Detection of TCS using an aptasensor}

In-solution detection of TCS was carried out using the aptamer-protected AuNPs constructed with TCS aptamer H6. Formation of the protected nanoparticles was performed as described in Section 5.2.2.3, except for that only one aptamer concentration (i.e. the optimal concentration) was chosen to protect AuNPs. The resulting AuNP aptasensor was utilised to detect TCS and structurally similar compounds, i.e. TCS, methyl-triclosan (MTS), bisphenol A (BPA) and oestradiol (OE2). 
These compounds were first prepared as stock solutions at $25 \mathrm{mM}$ in $100 \%$ ethanol. For sensing experiments, the stock solutions of the aforementioned compounds were diluted to appropriate working concentrations using $\mathrm{dH}_{2} \mathrm{O}$ containing $5 \%(\mathrm{v} / \mathrm{v}$ ) ethanol.

\subsubsection{In-solution detection of TCS}

Detection of TCS was carried out in six independent experiments. In a typical experiment, aliquots (each $100 \mu \mathrm{L}$ ) of the TCS-H6 aptasensor were added into microcentrifuge tubes that each contained $12 \mu \mathrm{L}$ of different concentrations of the TCS solution $(0-7.5 \mu \mathrm{M})$. The resulting solutions were incubated at RT for 15 mins to allow for dissociation of the aptamer from the surface of AuNPs. Thereafter, $8 \mu \mathrm{L}$ of $\mathrm{NaCl}$ solution at the optimal concentration was added to each tube for inducing the aggregation of AuNPs that were no longer protected by the adsorbed aptamer due to conformational change as a result of the aptamer binding to TCS. Following the addition of $\mathrm{NaCl}$ solution, the resulting solutions were sequentially subjected to the wristflick-incubation, photography and visualisation test. In order to obtain quantitative information for the sensing experiments, these samples were also subjected to UV-visible absorption spectroscopy using a NanoDrop 1000c Scan UV-visible spectrophotometer (Thermofisher Scientific, Waltham, Massachusetts, USA) with a spotting volume of $5 \mu \mathrm{L}$ per sample. Absorption peaks at 520 and $625 \mathrm{~nm}$ were utilised to obtain a standard curve for TCS detection by plotting the ratio of $625 / 520$ versus TCS concentration.

\subsubsection{In-solution detection of TCS structural similar compounds}

Detection of compounds sharing structural similarities with TCS (i.e. MTS, BPA and OE2) was carried out in three independent experiments. Each of these experiments was carried out as described in Section 5.2.3.1. In brief, aliquots (each $100 \mu \mathrm{L}$ ) of the TCS-H6 aptasensor were added into microcentrifuge tubes that each containing either $12 \mu \mathrm{L}$ of TCS solution at $7.5 \mu \mathrm{M}$ (as a reference) or one of the structurally similar compounds at the same concentration as TCS. A control sample containing $12 \mu \mathrm{L}$ of $\mathrm{dH}_{2} \mathrm{O}$ was also included in each experiment and treated the same as the tested samples. Thereafter, $\mathrm{NaCl}$ solution $(8 \mu \mathrm{L})$ was added to each of the control and test sampled. The remaining steps for detecting TCS structural similar compounds were performed the same as the detection of TCS. 


\subsection{Results}

\subsubsection{Spectral characteristics of the synthesised AuNPs}

The synthesised AuNPs were characterised with respect to their optical and physical properties by obtaining UV-visible adsorption spectra (Figure $5.2 \mathrm{~A}$ ) and a TEM image (Figure 5.2 B).

Figure 5.2: Spectral characteristics of the synthesised AuNPs (A) UV-visible adsorption spectra and; (B) a transmission electron microscope image

A.

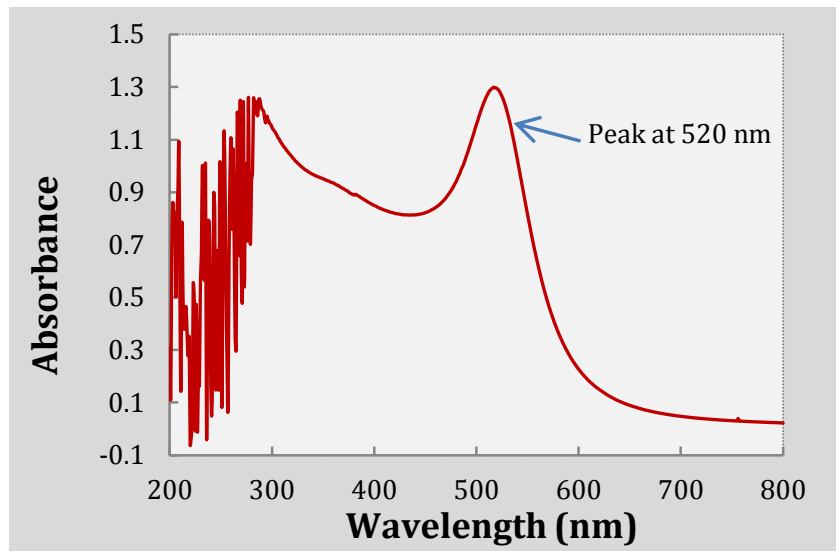

B.

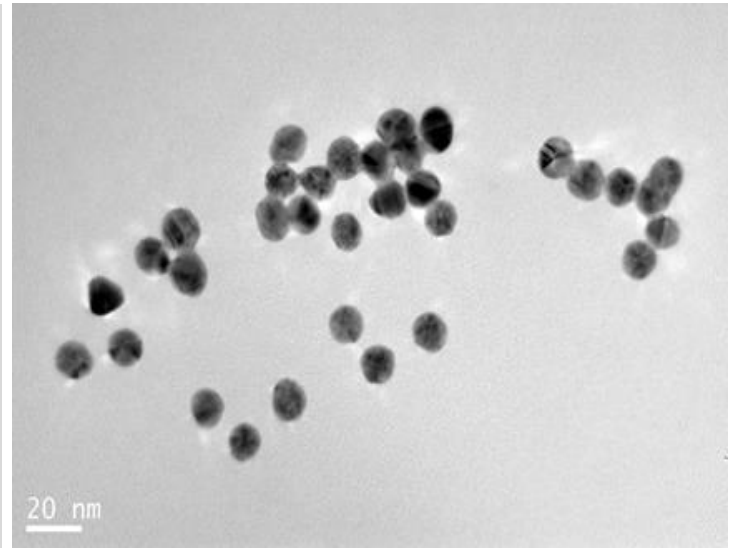

Based on the absorption spectra (Figure 5.2 A), the concentration of the synthesised AuNPs was approximately $13 \mathrm{nM}$ determined according to Beer-Lambert Law by using an absorption peak of 1.3 and the extinction coefficient of $2.01 \times 10^{8} \mathrm{M}^{-1} \mathrm{~cm}^{-1}$ at $520 \mathrm{~nm}$ (Maye et al. 2003). The absorption spectra also indicated that the AuNPs were monodispersed, since no shoulder peaks around $520 \mathrm{~nm}$ were observed. This was also confirmed by visualising the AuNPs under a TEM (Figure 5.2 B), and the diameter of each AuNP were estimated to be approximately $15 \mathrm{~nm}$.

\subsubsection{Optimisation of AuNPs for biosensing}

In this study, the $\mathrm{NaCl}$ tolerance test was performed by exposing the AuNPs to different concentrations of $\mathrm{NaCl}$ solutions (Figure 5.3). 
Figure 5.3: Salt tolerance test of the synthesised AuNPs

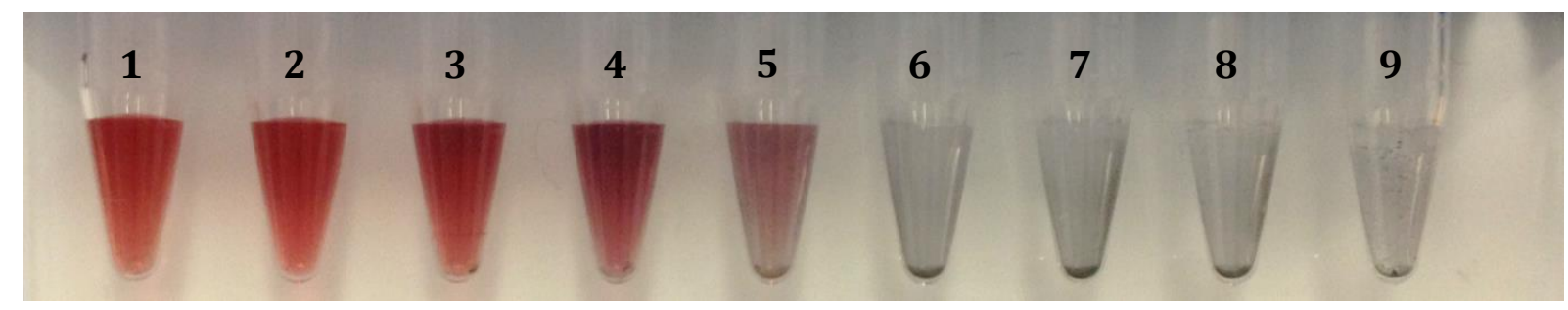

Samples 1 to 9 indicate the AuNPs with increasing $\mathrm{NaCl}$ concentrations of $0,6.7,13.3,20$, 26.7, 33.3, 40, 46.7 and $53.3 \mathrm{mM}$ respectively.

Only $13.3 \mathrm{mM} \mathrm{NaCl}$ was required to induce the AuNP aggregation with a noticeable colour change (Figure 5.3, sample 3), and from $26.7 \mathrm{mM} \mathrm{NaCl}$ onwards, the AuNPs become completely aggregated (Figure 5.3, samples 5 - 9). Therefore, $26.7 \mathrm{mM}$ was chosen as the optimal $\mathrm{NaCl}$ concentration to induce the aggregation.

The possibility of target-induced AuNP aggregation was excluded by exposing the AuNPs with TCS at different concentrations. The results demonstrated that TCS did not induce the aggregation of AuNP up to $2 \mu \mathrm{M}$ (Figure 5.4).

Figure 5.4: The effect of increasing concentrations of TCS in the presence of AuNPs

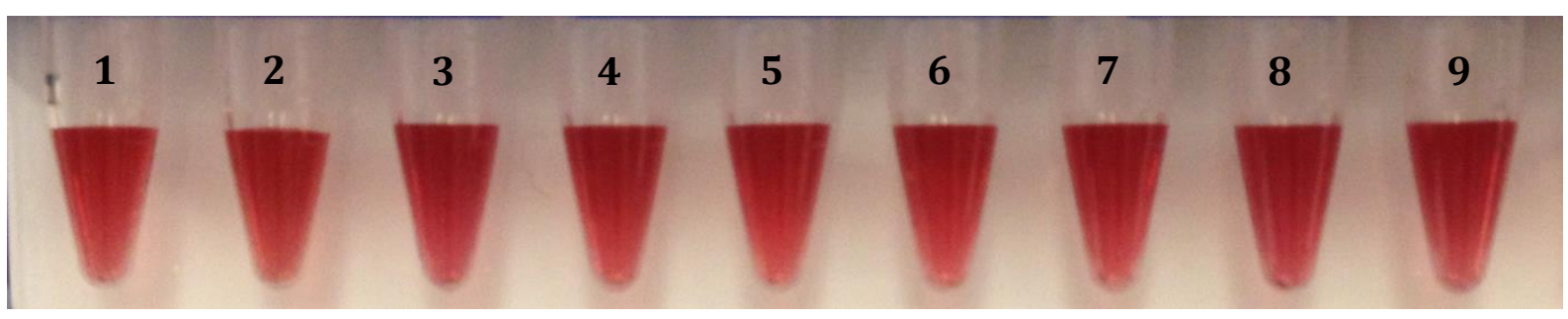

Samples 1-9 indicate the AuNPs with added TCS at 0, 20, 50, 150, 300, 500, 750, 1000 and $2000 \mathrm{nM}$ respectively.

In order to prepare AuNPs for TCS detection, an aptamer need to be coated on the surfaces of AuNPs to form a protective layer to prevent $\mathrm{NaCl}$-induced aggregation. The appropriate amount of the aptamer required for this protection was determined by coating the AuNPs with different amount of TCS aptamer H6, and the resulting aptamer-protected AuNPs were then subjected to $\mathrm{NaCl}$-induced aggregation at the optimal $\mathrm{NaCl}$ concentration (Figure 5.5). 
Figure 5.5: Optimisation of the amount of the H6 aptamer required for protecting AuNPs against $26.7 \mathrm{mM}$ salt-induced aggregation

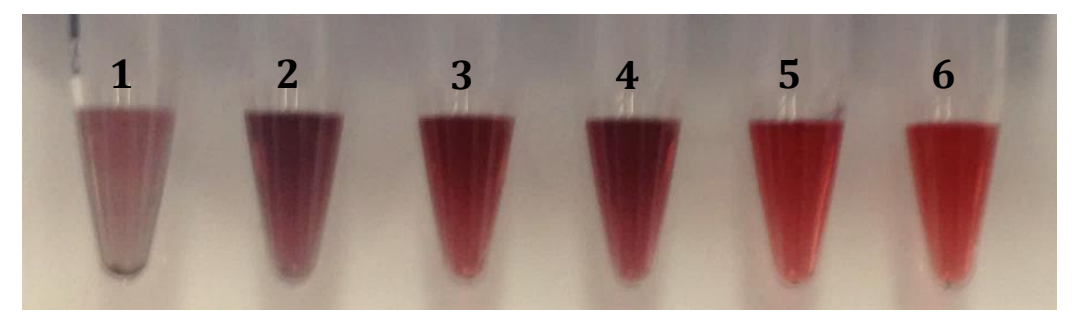

Samples 1 to 6 indicate the AuNPs are protected by increasing concentrations of the TCSH6 aptamer from 0, 28, 56, 84, 112 and $140 \mathrm{nM}$ respectively.

The use of $26.7 \mathrm{mM} \mathrm{NaCl}$ caused complete aggregation of AuNPs when no aptamer was present to protect the AuNPs (Figure 5.5, sample 1). Partial aggregation was observed at 28, 56, and $84 \mathrm{nM}$ aptamer concentrations (Figure 5.5, samples 2-4). No visible aggregation was observed at 112 and $140 \mathrm{nM}$ aptamer concentrations (Figure 5.5, samples 5 and 6). Therefore, $112 \mathrm{nM}$ was chosen as the optimal aptamer concentration.

\subsubsection{Detection of TCS and structurally similar compounds}

After optimising the various components (i.e. salt tolerance, target effect and aptamer concentration), the TCS aptasensor was investigated with the respects of sensitivity and specificity to TCS and structurally similar compounds.

\subsubsection{Testing the sensitivity of TCS aptasensor}

TCS aptasensor was applied for in-solution detection of TCS at final concentrations ranging from 0 to $750 \mathrm{nM}$. Qualitative analysis (observing the visual colour change by naked-eye) was performed and an image was captured (Figure 5.6a).

Figure 5.6a: Qualitative detection of TCS by observing colour changes due to the formation of AuNP aggregates

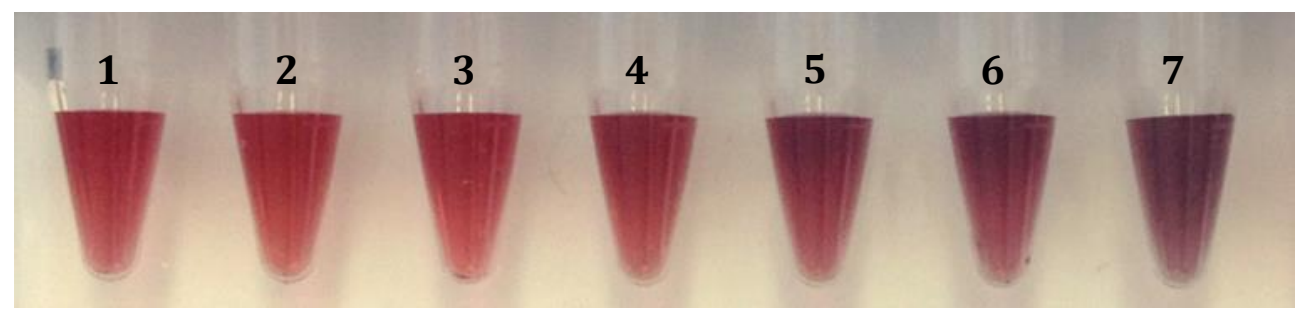

Samples 1-7 indicate the TCS-H6 aptasensor with added TCS at 0, 20, 50, 150, 300, 500, and $750 \mathrm{nM}$ respectively. 
A noticeable colour change was evident from 150 nM TCS onwards (Figure 5.6a, sample 4). Quantitative analysis (measuring the absorption spectra using a nanodrop spectrophotometer) was undertaken to confirm the visual observations (Figure 5.6b).

Figure 5.6b: Quantitative UV-visible spectrophotometric detection of TCS in solution using the H6 aptamer-protected AuNPs

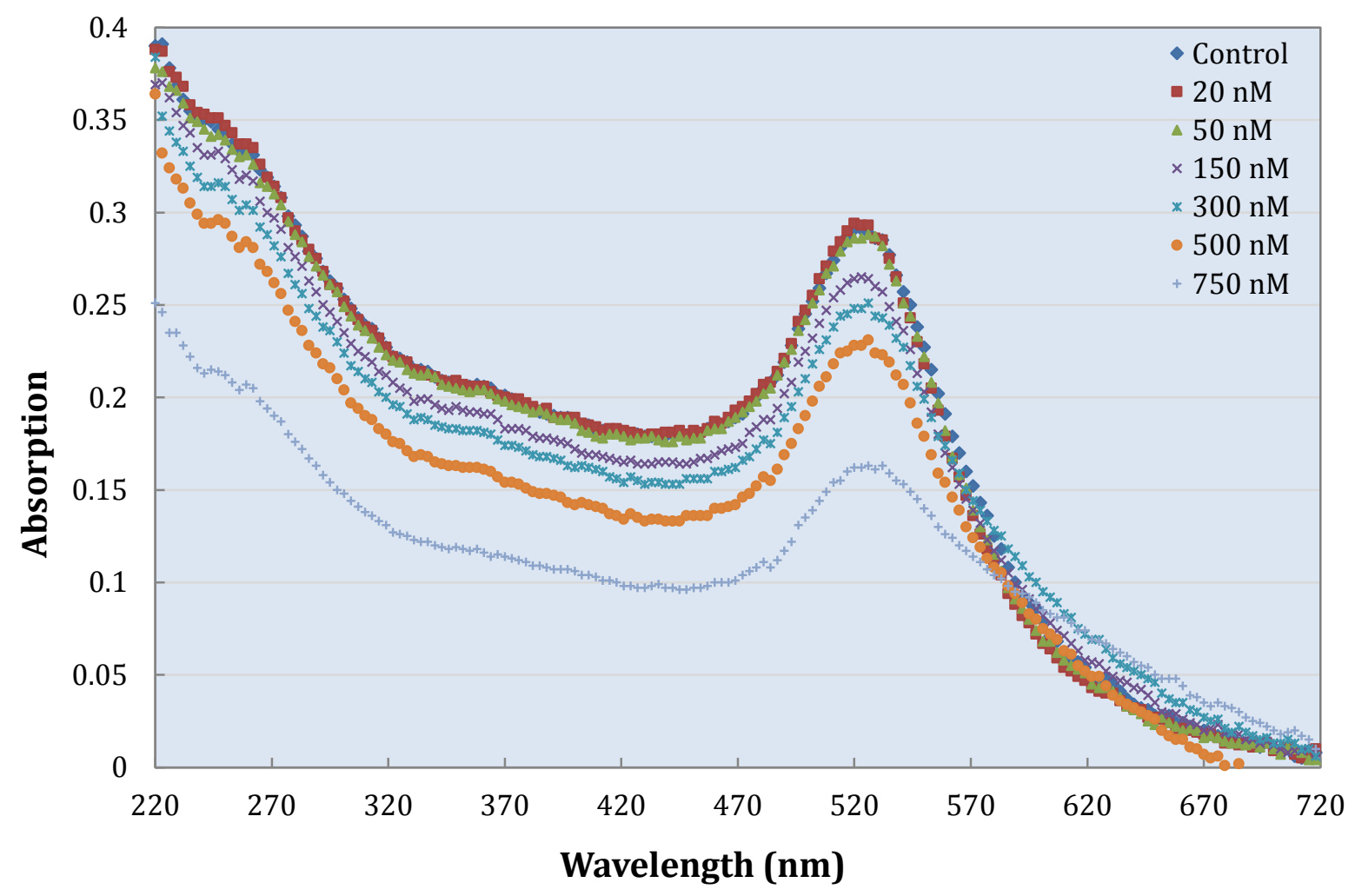

The seven spectra are for the control (no TCS) and six concentrations of TCS (20-750 nM).

The absorption peaks at $520 \mathrm{~nm}$ wavelength decreased with increasing concentrations of TCS (Figure 5.6b) which is in agreement with the visual observation (Figure 5.6a).

The quantitative detection capability of the TCS aptasensor was further characterised by determining the relationship between absorption ratios (A625nm/A520nm) over the range of TCS concentrations tested (Figure 5.6c). 
Figure 5.6c: Correlation between the absorption ratio and the concentration of TCS (20$750 \mathrm{nM})$

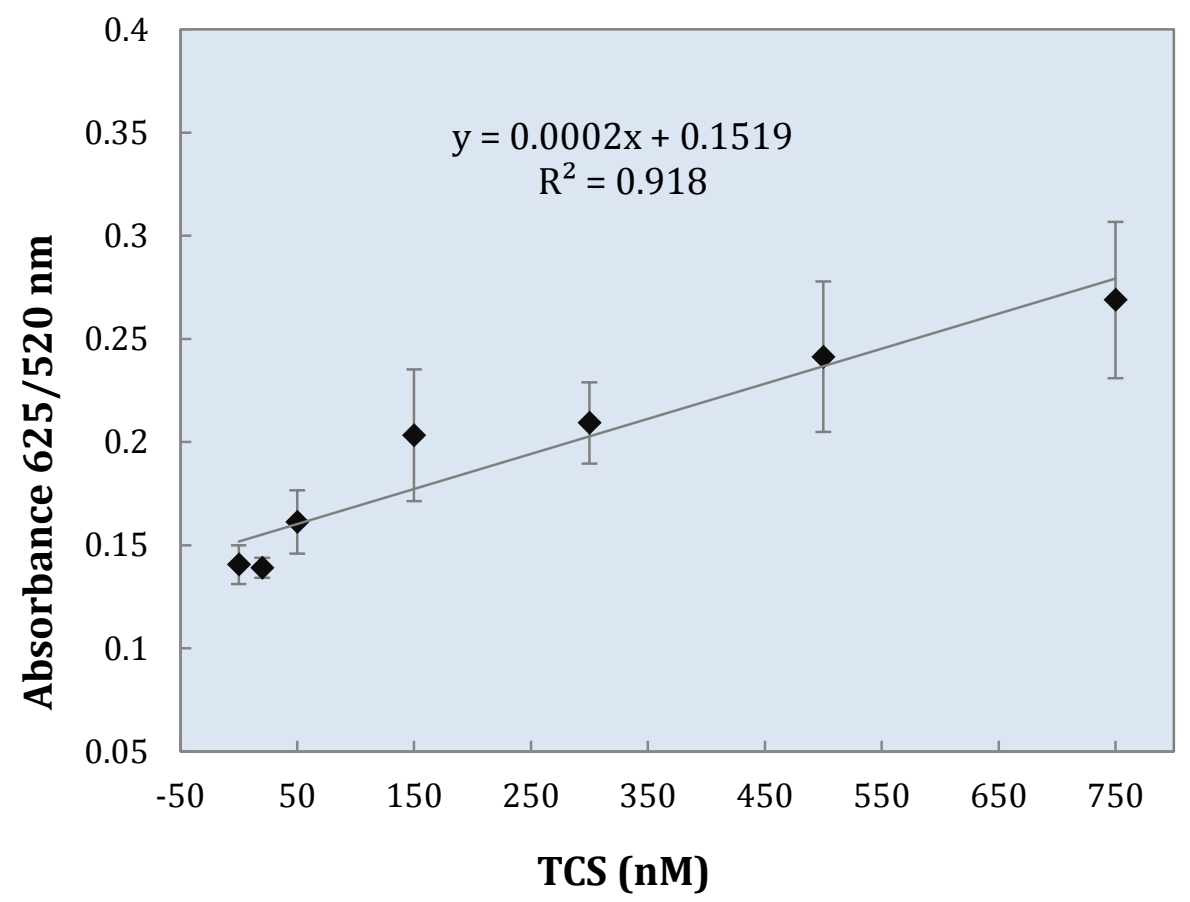

The error bars represent the standard deviation of the mean absorbance ratio from six independent sensing experiments.

A linear correlation between the absorption ratio and TCS concentration was observed with an $\mathrm{R}^{2}$ value of 0.918 (Figure 5.6c). However, the error bars appear large, analysis of the variation in the calculated slopes indicates the sensitivity of this assay is quite reproducible and therefore validating the applicability of the aptasensor to measure TCS from 20 to $750 \mathrm{nM}$.

\subsubsection{Testing the specificity of TCS aptasensor}

The specificity of the TCS aptasensor was tested by using samples spiked with MTS, BPA or OE2 to obtain a final concentration of $750 \mathrm{nM}$. Visual analysis was undertaken and the image was captured (Figure 5.7a). 
Figure 5.7a: Qualitative determination of the specificity of TCS aptasensor

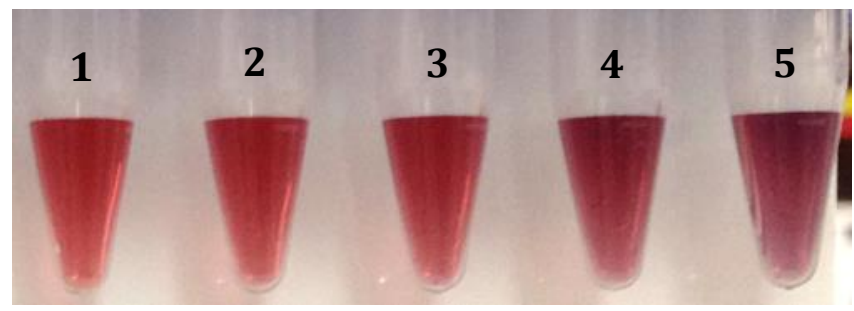

The samples 1-5 indicate $750 \mathrm{nM}$ solution of the control, OE2, BPA, MTS and TCS respectively.

Visual analysis (Figure 5.7a) showed no significant changes in colour when the OE2 (sample 2) and BPA (sample 3) were introduced compared to the control (sample 1). Whereas a slight change in colour was observed when tested with MTS (sample 4). The aptasensor showed significant changes in colour when TCS (sample 5) was introduced as a positive control. Spectral analysis of the samples were undertaken using nandrop spectrophotometer (Figure 5.7b).

Figure 5.7b: Quantitative UV-visible spectrophotometric detection of the specificity of TCS aptasensor

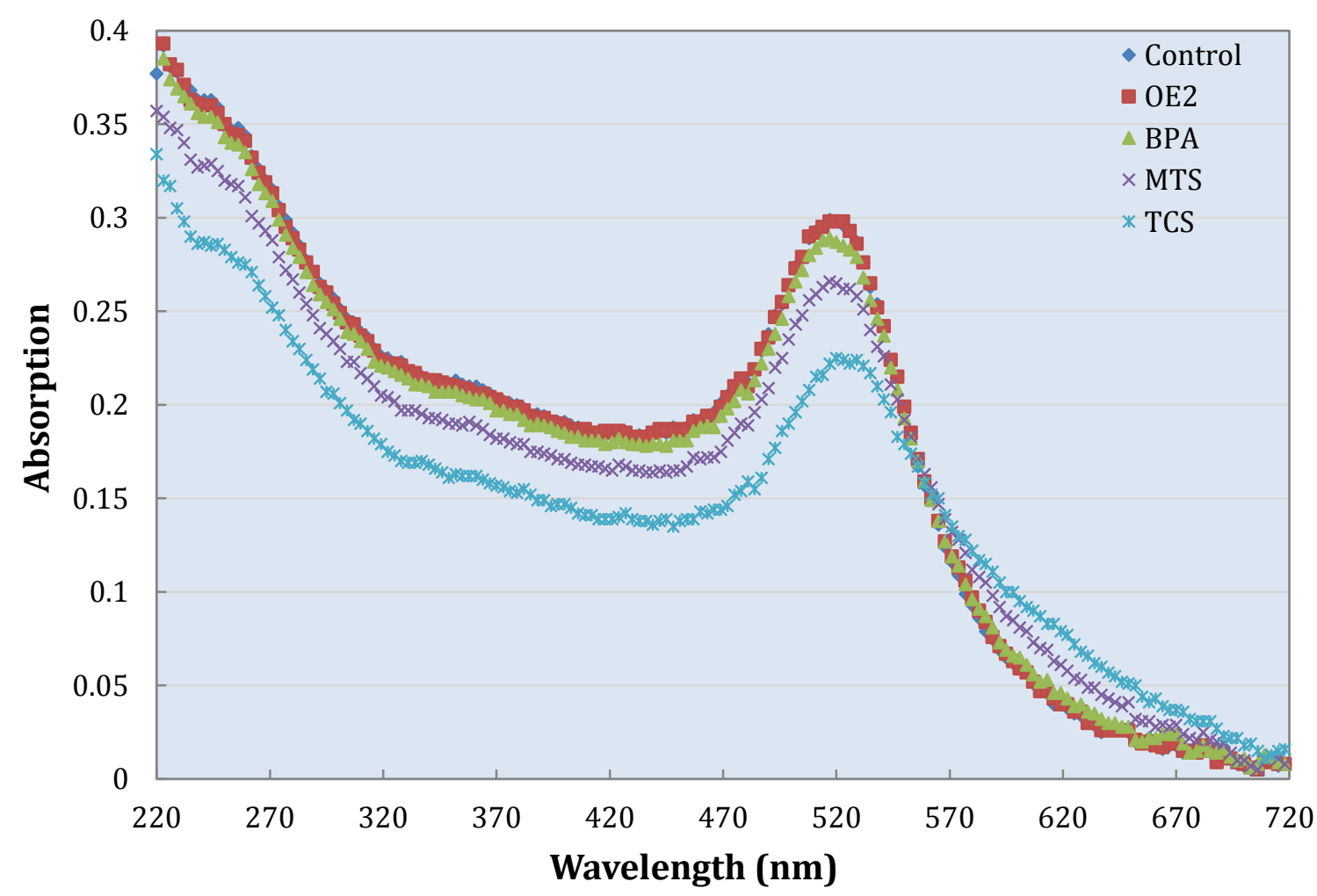

The five absorbance spectra represent the control and four compounds tested (i.e. TCS, MTS, BPA and OE2) at $750 \mathrm{nM}$. 
The UV-visible absorption spectra confirmed the visual observations (Figure 5.7b). The absorption spectra of OE2 and BPA showed a similar height as the control sample at $520 \mathrm{~nm}$, whereas, a reduced height observed on MTS when compared with the control sample demonstrating some non-specific interaction of the H6 aptamer with MTS.

The specificity data of the TCS aptasensor was also illustrated by plotting a relative response graph (Figure 5.7c).

Figure 5.7c: Non-specific responses of TCS aptasensor to compounds (MTS, BPA and OE2) that are structurally similar to TCS

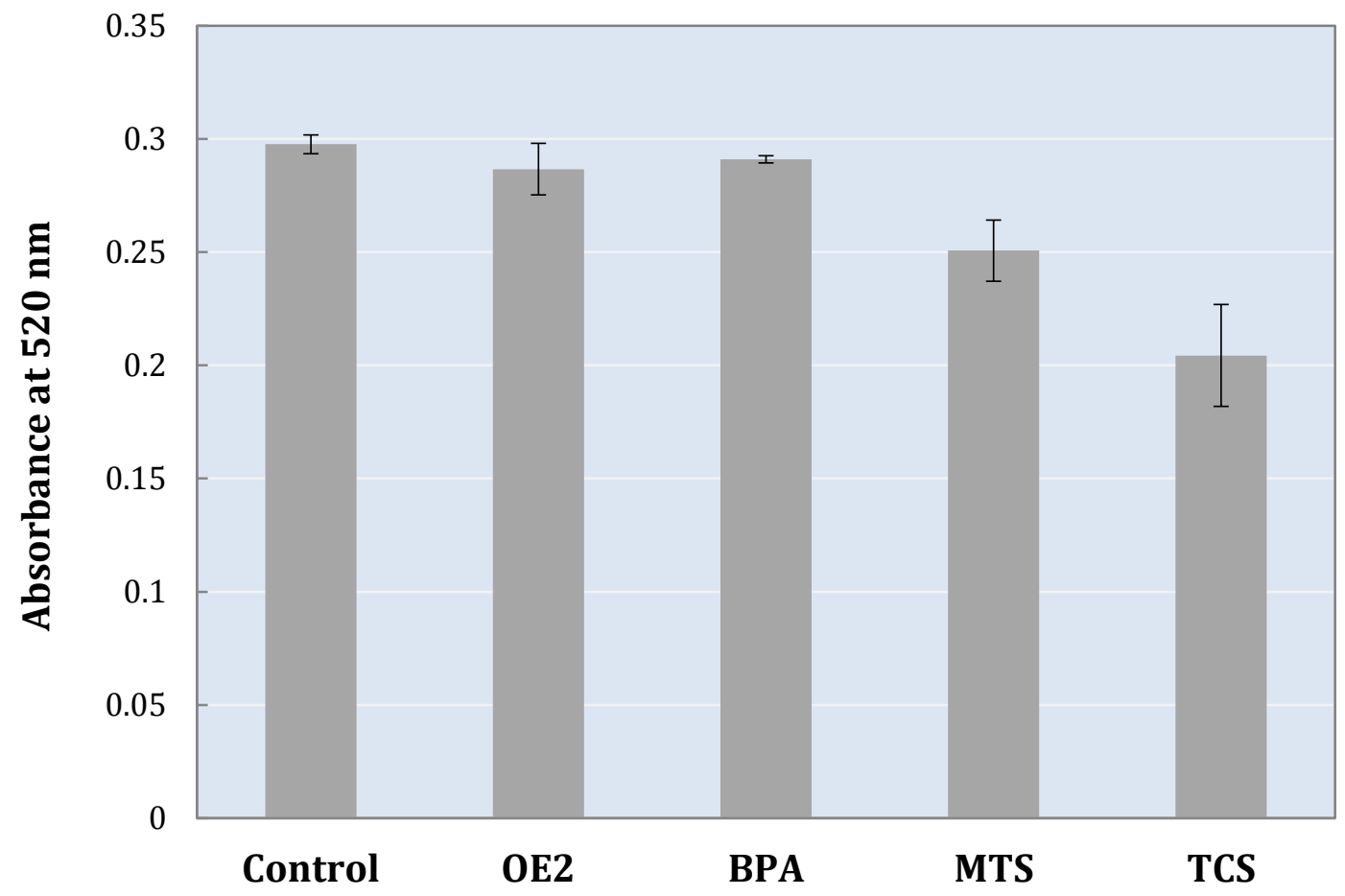

The error bars represent the standard deviation of the reduced absorption from three independent sensing experiments.

The TCS aptasensor showed no non-specific interactions with OE2 and BPA, but some interaction with MTS, for which the response was about half of that observed with TCS. 


\subsection{Discussion}

In this chapter, a novel AuNP-based aptasensor was developed to detect TCS in-solution using the $\mathrm{H} 6$ aptamer generated in this dissertation. The synthesised AuNPs were first characterised using UV-visible spectrophotometer and TEM.The results showed that the AuNPs were monodispersed (Figure 5.2). This was considered to be a critical factor, as monodispersed-AuNPs ensure maximum homogeneity of aptamer adsorption, thereby allowing a uniform surface plasmon coupling and quantitative analysis of the AuNPs using UV-visible spectroscopy.

During the development of the aptasensor, the optimisation steps (i.e. salt and aptamer concentrations etc) validated the application of this system for detecting TCS. Distinguishable colour changes in TCS containing samples enabled the qualitative determinations of TCS with a LOD of $150 \mathrm{nM}$ by naked-eye using the TCS aptasensor. UVvisible absorption peaks at 520 and $625 \mathrm{~nm}$ also enabled the quantitative determination of the TCS molecule in solution. Analyses of the absorption spectra revealed a linear correlation $\left(R^{2}=0.918\right)$ at a concentration range of TCS from 20-750 nM (Figure 5.6c). These results are comparable to those obtained from the study by Alsagar et al. (Alsager et al. 2015). In their study, the use of a full length 75-mer OE2 aptamer-protected AuNPs demonstrated colorimetric sensitivity for OE2 in the nanomolar range from 5 to 400 with acceptable linearity $\left(R^{2}=0.92\right)$ and naked-eye detection around $100 \mathrm{nM}$. In addition, they also demonstrated that the length of the OE2 aptamer used for protection is often important, based on the fact that two specifically truncated OE2 aptamers ( 35 and 22 mer) showed greater sensitivity down to picomolar range compared with the full length version. They reasoned that the full length aptamer might not be able to fully dissociate from the AuNP surface, and part of the sequences were still adhered to the surface. Based on this discovery, the 75-mer $\mathrm{H} 6$ aptamer was also subjected to truncation by simply omitting the primer regions. Unfortunately, the small versions of the $\mathrm{H} 6$ aptamer obtained showed significant reduction in their binding affinity to TCS (Figure 4.11b, Chapter 4), and therefore were not suitable for the sensor development. However, it is reasonable to assume that the detection range of the TCS aptasensor may be improved if the H6 aptamer can be specifically truncated to leave only the TCS binding pocket. 
The specificity of the TCS aptasensor is dependent on the capability of the H6 aptamer to selectively bind to TCS with minimal cross-reactivity to structurally similar compounds such as OE2, BPA and MTS. The results from the binding specificity experiments showed that the H6 aptamer showed little interaction with OE2 and BPA at $750 \mathrm{nM}$. However, the aptamer did display some binding affinity to MTS. This is reasonable as MTS is only one methyl group difference compared with TCS, and the aptamer H6 was unable to differentiate such a small difference between the two molecules. It's worth noting that aptamer H6 was selected without any counter selection against MTS. These results are comparable to those from the competitive binding studies (Figure 4.10b, Chapter 4) indicating that the H6 aptamer is specific to TCS.

In summary, initial sensing results obtained from the TCS aptasensor suggest that the H6 aptamer is suitable for the development of an aptasensor, and that the aptasensor can detect TCS in the nanomolar range with some specificity against related organic compounds (e.g. MTS). These assured the future applicability of the TCS aptasensor for detecting TCS in a free solution environment.

\subsection{Conclusion}

The work presented here introduced a simple and reliable colorimetric aptasensor for the detection of TCS using the H6 aptamer as the recognition element and the AuNPs as the signal transducer, with a visual detection limit of $150 \mathrm{nM}$ and with some proven discrimination against structurally similar molecules. The developed aptasensor is capable of providing both qualitative and quantitative detection of TCS. The conformational change of the aptamer upon binding to the target could be reflected in the colour change of AuNPs, and thus the detection of TCS could be realised by naked eye. Furthermore, it is suggested that by finding the target binding pocket of the H6 aptamer, specifically truncated H6 aptamers could further enhance the sensitivity and specificity of the TCS aptasensor. Therefore, it is concluded that the AuNP-based aptasensors could be used for the detection of small molecules such as TCS, and further the use of the aptasensor could provide a simple diagnostic tool for future on-site screening of EDCs. 


\section{CHAPTER 6}

\section{GENERAL DISCUSSION AND FUTURE PERSPECTIVES}

Aptamers have been proven to be highly versatile reagents for biological and diagnostic applications ever since the concept was developed in 1990. A multitude of analytical and therapeutic usages of aptamers have been reported, and the number of aptasensors reported in the literature have increased exponentially in recent years. In this dissertation, novel methodologies were developed and utilised in the SELEX technology to produce the first known aptamers capable of binding to TCS, and an AuNP-based aptasensor was developed for detecting TCS in spiked-water samples. The following provides a general discussion for each method used in the SELEX procedure. Also discussed is the application of in silico methods for identifying regions of interest within an aptamer sequence that maybe responsible for the association with its target.

\subsection{Affinity matrix}

Preparation of target-immobilised affinity matrices is essential for selecting aptamers against small moleculars. In this study, to allow for easy separation of target-bound aptamers from unbound oligonucleotides, TCS was chemically conjugated onto the surface of sepharose beads using linker molecules with different length in their linker arms (Figure 3.2, Chapter 3). As a result, the proximity of TCS from the bead moieties increased, thereby allowing for the maximum exposure of the target to associate with random oligonucleotides during selection. Results from Chapter 3 (Figure 3.5 and 3.11) demonstrated that the long-arm TCS D matrix was more suitable than the short-arm TCS E matrix for aptamer selection. This finding indicated that the length of a linker molecule used for target immobilisation might influence the efficiency of a SELEX experiment. Therefore, it is concluded that determining the appropriate length of a linker molecule used for conjugation is an important factor to be considered in future SELEX experiments for selecting aptamers capable of binding small molecules. During the preparation of affinity matrices, the remaining epoxy groups after TCS immobilisation were blocked using ethanolamine. Blocking these groups is necessary as they are potentially reactive with either oligonucleotides or proteins presented in the selection buffers. 
However, the blocking step may introduce alternative recognition sites for association with aptamers. Therefore, the reagent used for blocking needs to be as small as possible to minimise the non-specific interaction with oligonucleotides during selection. Conjugations of TCS onto the surface of a matrix were confirmed by the presence of the absorption peaks on the conjugated matrices (Figure 3.3, Chapter 3). UV-visible spectroscopy was utilised to obtain the absorption spectra. The method can provide qualitative measure (i.e. a successful conjugation), as well as quantitative determination of the amount of immobilised TCS molecules provided the extinction coefficient value is known. Determination of the extinction coefficient value was not performed in the present study. However, it is recommended in future selection work, as knowing the exact amount of immobilised-target can reduce the inconsistency during each cycle of selection and increase the accuracy for aptamer characterisation.

\subsection{SELEX selection}

The present study demonstrated the use of both basic- and revised-SELEX procedures for selecting TCS-specific aptamers. The basic-SELEX procedure is the most commonly used approach for selecting aptamers binding to small molecules. Aptamers (i.e. D and E clones) with considerable binding affinities to TCS were identified from this approach. However, these aptamers demonstrated significant levels of non-specific binding to the matrix, and some of the aptamers also showed evidence of interacting with structurally similar compounds (Figure 3.10 and 3.11, Chapter 3). Therefore, it was concluded that more than eight rounds of selection were required to generate aptamers with sufficient sensitivity to TCS when using the basic-SELEX methodology. However, the selection process was in practice restricted to 8 rounds, as further rounds of selection led to the formation of nonspecific PCR by-products (Figure 3.4, Chapter 3). This led to a revised-SELEX procedure being developed, and the resulting aptamers (H1 - H10) from the revised-procedure were found to have improved binding affinity and specificity (Figure 4.8b and 4.10b, Chapter 4), indicating that the introduced modifications (i.e. tRNA for matrix blocking, negative and counter selections, and real-time amplification) were effective in minimising the non-specific adsorption between oligonucleotides and the matrix, eliminating non-specific oligonucleotide sequences binding to structurally similar molecules and reducing the formation of non-specific PCR by-products. 
Therefore, the revised-SELEX cycle can be repeated beyond 8 rounds of selection, if necessary. The ability to carry out further rounds of selection would enable in the refinement of aptamers to attain desired target-binding characteristics. As a result, these modifications were considered to be suitable strategies for future SELEX experiments. Table 6.1 summarises the criteria from the revised-SELEX procedure which are suggested to be effective for selecting aptamers to small molecules.

Table 6.1: Summary of methods and conditions used for aptamer selection

\begin{tabular}{|c|c|c|}
\hline SELEX step & Recommend & Alternative \\
\hline Affinity matrix & Sepharose beads & $\begin{array}{l}\text { Agarose beads } \\
\text { Magnetic beads }\end{array}$ \\
\hline $\begin{array}{l}\text { Affinity matrix } \\
\text { characterisation }\end{array}$ & UV-visible characterisation & \\
\hline Aptamer library & 75-mer with 40 random region & \\
\hline Design primers & $\begin{array}{l}\text { Oligo Analyzer Software } \\
-\quad 18 \text {-mer forward primer } \\
-\quad \text { 17-mer reverse primer }\end{array}$ & \\
\hline SELEX buffers & $\begin{array}{l}\text { 1X BWB } \\
-\quad 100 \mathrm{mM} \mathrm{NaCl}, 5 \mathrm{mM} \mathrm{KCl}, 2 \mathrm{mM} \mathrm{MgCl} \text {, } \\
\\
\quad 1 \mathrm{mM} \mathrm{CaCl}, 20 \mathrm{mM} \text { Tris HCl, and } 0.1 \% \\
\text { (v/v) IGEPAL CA } 630 \text { (pH 7.5) } \\
\text { Blotto } \\
-\quad 10 \mathrm{mg} / \mathrm{mL} \text { yeast tRNA } \\
-\quad 5 \%(\mathrm{v} / \mathrm{v}) \text { skim milk power }\end{array}$ & \\
\hline SELEX preparation & $\begin{array}{l}\text { Matrix blocking } \\
-\quad 2 \text { hrs blocking at RT } \\
\text { Aptamer library denaturation } \\
-\quad 90^{\circ} \mathrm{C} \text { for } 10 \text { mins, } 4^{\circ} \mathrm{C} \text { for } 15 \text { mins, and } \\
10 \text { mins at RT }\end{array}$ & \\
\hline Selection strategy & $\begin{array}{l}\text { Negative selection } \\
-\quad \text { after two rounds of positive selection } \\
\text { Counter selection } \\
-\quad \text { after negative selection }\end{array}$ & \\
\hline $\begin{array}{l}\text { Recover bound } \\
\text { aptamers }\end{array}$ & Not necessary & $\begin{array}{l}\text { Affinity based elution } \\
\text { Heat-denaturing }\end{array}$ \\
\hline Amplification & Real-time PCR amplification & $\begin{array}{l}\text { Emulsion PCR } \\
\text { Digital PCR }\end{array}$ \\
\hline $\begin{array}{l}\text { Purification of PCR } \\
\text { products }\end{array}$ & $\begin{array}{l}\text { Agarose gel electrophoresis } \\
-\quad 3 \%(\mathrm{w} / \mathrm{v})\end{array}$ & PAGE \\
\hline Strand separation & $\begin{array}{l}\text { Alkaline denaturation } \\
-\quad \text { biotin-streptavidin }\end{array}$ & $\begin{array}{l}\text { Asymmetric PCR } \\
\text { Lambda exonuclease }\end{array}$ \\
\hline SELEX progression & Fluorescent imaging assay & $\begin{array}{l}\text { Dot blot assay } \\
\text { Affinity column elution }\end{array}$ \\
\hline $\begin{array}{l}\text { Cloning and } \\
\text { sequencing }\end{array}$ & At least 10 clones to be sequenced & \\
\hline
\end{tabular}

PAGE = polyacrylamide gel electrophoresis; UV-visible = ultraviolet-visible; $\mathrm{BWB}=$ binding and washing buffer; Blotto = blocking buffer; $\mathrm{PCR}=$ polymerase chain reaction and; $\mathrm{RT}=$ room temperature. 


\subsection{Aptamer binding characterisation}

A significant outcome from this study was the development of a novel fluorescent imaging assay to determine the fluorescent intensity of the HEX-labelled aptamer-bound matrices. The developed mehod was used for monitoring SELEX progression, as well as determining aptamer binding affinity ( $K_{d}$ values) and specificity. In addition, the applicability of the assay was compared with the dot blot and fluorescent binding assays, and results from all the assays were comparable with each other, thereby validating the use of the fluorescent imaging assay for aptamer characterisation (see Chapter 4). Table 6.2 summarises the results of using both the fluorescent imaging and binding assays for aptamer $K_{d}$ determination.

Table 6.2: Summary of $K_{d}$ values demonstrated by TCS-specific aptamers

\begin{tabular}{ccc}
\hline Aptamer & $\begin{array}{c}\text { Fluorescent imaging assay } \\
\text { Mean } \pm \text { SD }(\mathbf{n M})\end{array}$ & $\begin{array}{c}\text { Fluorescent binding assay } \\
\text { Mean } \pm \text { SD (nM) }\end{array}$ \\
\hline H2 & $1448 \pm 50$ & $>1000$ \\
H5 & $604 \pm 85$ & $544 \pm 107$ \\
H6 & $378 \pm 50$ & $369 \pm 82$ \\
D5 & $584 \pm 251$ & $700 \pm 174$ \\
E6 & n/a & $>1000$ \\
\hline
\end{tabular}

$\mathrm{SD}=$ standard deviation; H2, H5 and H6 are aptamers from the revised-SELEX and; D5 and E6 are aptamers from the basic-SELEX.

The major advantages of the fluorescent imaging assay include: [i] no requirement to elute aptamers once bound to the target-conjugated matrices, thereby eliminating the uncertainty of not achieving a complete elution; [ii] simple-to-use, as it requires fewer preparation steps when compared with the dot blot and florescent binding assays; [iii] reproducibility, as the present study showed that after replicating the $K_{d}$ experiments five times, the standard errors were low (Table 6.2) and; [iv] the simple application of visual detection of the fluorescent images. It is also worth noting that the fluorescent imaging assay is not restricted for characterising aptamers for small molecules. Aptamers capable of binding to protein targets can also be characterised using this method, since proteins can easily be immobilised onto the surface of a matrix. The only drawback for the imaging method is the analytical procedure. 
Currently, ROI for each image are manually selected. This can be time-consuming, and open to potential errors when selecting different ROI for analysis. However, with the current computer technologies, an automated image analysis process would not be difficult to develop. Therefore, the fluorescent imaging assay has great potential for becoming a standard procedure for aptamer development.

\subsection{Aptamer in silico characterisation}

In silico characterisation of aptamers can be undertaken using a range of techniques, enabling the investigator to identify specific regions of interest within each aptamer sequence. The selective conditions being applied during SELEX, generally enriches the nucleotide pool with aptamers having an improved affinity and/or specificity towards the target molecule. Therefore, analysis of the nucleotide sequences within the random region of an aptamer with respect to nucleotide composition, phylogenetic relationship, sequence alignment, and secondary structure prediction, can potentially provide insights into the evolutionary process and identify key nucleotides that may involve in the target binding site.

\subsubsection{Nucleotide composition}

TCS R8 aptamers generated from the basic-SELEX procedure were composed of a relatively high degree of $\mathrm{G}$ and $\mathrm{T}$ nucleotides with $\mathrm{T}$ being the most abundant occupying over $30 \%$ of the N40 region (Table 3.2, Chapter 3). TCS R10 aptamers generated using the revised-SELEX procedure also showed an enrichment of $\mathrm{G}$ and $\mathrm{T}$ nucleotides, but with $\mathrm{G}$ being the most abundant nucleotides occupying over $40 \%$ of the $\mathrm{N} 40$ region (Table 4.4 , Chapter 4). This change in the nucleotide composition within the random region could be attributed to the fact that more selection rounds were performed in the revised-SELEX procedure, and therefore more evolutionary pressure was applied in the nucleotide pool to direct the selection of more stable nucleotide combinations when compared with the basic-SELEX procedure. In contrast to the double hydrogen bonding between $\mathrm{A}$ and $\mathrm{T}$ nucleotides, the $\mathrm{G}$ and $\mathrm{C}$ complementary pairing offers a triple bonding alternative which requires higher energy to dissociate (Kang et al. 2005). Thus, an increase in the number of G over T nucleotides in the R10 aptamers is likely to favor the formation of more stable aptamer 3D structures. 
The generation of G-rich aptamers from the revised-procedure is similar to other reports demonstrating G-rich aptamers for small molecules such as the DNA aptamers for OTA (47\% of G) (Cruz-Aguado and Penner 2008), ATP (45 \% of G) (Huizenga and Szostak 1995), and cocaine (33 \% of G) (Stojanovic et al. 2001).

\subsubsection{Phylogenetic relationship}

The evolutionary pathways of TCS R8 and R10 aptamers were analysed from their phylogenetic trees. It was evident that the TCS D aptamers (i.e. D1, D2, D4, D5 and D6) were not closely related to the E aptamers (i.e. E1, E2, E3, E5 and E6), despite being subjected to identical selection pressures (Figure 3.6, Chapter 3). This was reasonable, as different selection matrices (i.e. TCS D and E matrices) were used for generating these aptamers. An interesting observation was the distribution of individual aptamers within each lineage (Figure 3.6, Chapter 3). For example, aptamers generated using the long-arm matrix were spread over a considerable distance from one another indicating diversity within the identified aptamers. In contrast, aptamers generated using the short-arm matrix clustered more closely within each other. Similarly, when using the long-arm matrix to select aptamers from the revised-procedure. The resulting aptamers (H1-H10) showed variable evolutionary distance within the phylogenetic tree (Figure 4.4, Chapter 4). These observations indicated that aptamers produced using a long-arm matrix had a relatively higher degree of diversity compared with the short-arm matrix thereby increasing the chance of identifying aptamers with different binding sites, whereas the use of short-arm matrix restricted the recognition of the target molecule by aptamers possessing different binding characteristics. This finding provides another insight in favour the use of long-arm matrix for aptamer selection. In addition, the analysis of phylogenetic trees indicated aptamers with desired binding properties to TCS (i.e. aptamers D5 and E6 from the basic-procedure and; aptamers H2, H5 and H6 from the revised-procedure) are generally evolved further than those aptamers with poor binding properties (Figure 3.6, Chapter 3; Figure 4.4, Chapter 4). Furthermore, aptamers with similar nucleotide compositions were found to be more closely related to each other, such as aptamers E1 \& E6 (Table 3.2 and Figure 3.6, Chapter 3), H4 \& H7 and H1 \& H5 (Table 4.4 and Figure 4.4, Chapter 4). 
In summary, analyses of the nucleotide composition and phylogenetic trees discussed above is informative in helping to understand the impact of different SELEX strategies on a pool of aptamers selected for a particular target.

\subsubsection{Sequence alignment and secondary structure prediction}

Characterisation of aptamer sequences based on the use of sequence alignment and secondary structure prediction can lead to the identification of conserved nucleotide domains (Niazi et al. 2008), and sometimes assist in identifying the target binding pocket (Lin and McNatty 2009).

In the present study, random regions of the two selected aptamer families (i.e TCS R8 and R10 aptamers) were individually aligned to search for regions of homology. In the case of aligning TCS R8 aptamers, there were no obvious conserved regions identified within each sequence (Figure 3.7, Chapter 3), but duplicate, triplicate and even quadruplet copies of T and G nucleotides, as well as duplicates and triplicates of A nucleotides were common features. Since only eight rounds of selection were performed in generating the R8 aptamers, it was not surprising that no conserved sequences were found during sequence alignment. In contrast, when aligning TCS R10 aptamers for the same purpose (Figure 4.5, Chapter 4), some conserved sequences were identified. These included: "GGTTX" (where X can be either A, T, C or G), "XGGGX" (where X can be both T, both A or C and T), "GGGGX" (where X can be either A or T"), and "TGGGGGX" (where X can be either $\mathrm{C}$ or A). At this stage, it is inconclusive as to whether these conserved sequences are part of the target binding site without confirming: [i] whether these regions of interest can contribute to the formation of either stems, loops or bulges, which are common features found in aptamer 3D structures and; [ii] aptamers containing these conserved sequences can actually bind to the target molecule. Therefore, the next step of in silico characterisation is to predict 3D structures of aptamers.

It is well established that aptamers bind to their target molecules by forming specific 3D structures such as hairpins (i.e. a combination of stems, loops or bulges), pseudoknots, or G-quadruplexes. However, in silico analysis of aptamer 3D structures has not yet been developed. A major factor limiting the development of such an in silico tool is the flexibility of ssDNA molecules which complicates the calculation for algorithms for computational modelling. 
Since the formation of aptamer 3D structures are dependent on aptamer primary sequences (Takemura et al. 2006), it was proposed that, using aptamer 2D structures, an accurate assessment of regions of interest within each aptamer and their potential role in binding to the target molecule might be elucidated. It also should be mentioned that: [i] the molecular structure of TCS is much smaller than an aptamer, thus only limited regions of a TCS-specific aptamer are likely to be involved in binding to TCS and; [ii] the mFold software is able to predict 2D secondary structures of ssDNA molecules containing multiple stem-loops (SL) in their lowest energy states. Therefore, it could be argued that the SL regions might assist in forming the most energetically stable structures necessary for an aptamer binding to its target. Based on these rationales, secondary structures of TCS R8 and R10 aptamers are each predicted and summarised in Appendix II and IV respectively. Nucleotides involved in the formation of SL structures were investigated.

Initial analysis of these secondary structures indicated a variety of stem-loop configurations ranging from one linear stem-loop structure (e.g. D1 and H7) up to four stem-loop structure (e.g. D4 and H5). Amongst these structures, aptamers D5 and E6 from the basic-SELEX selection, as well as H2, H5 and H6 from the revised-SELEX selection showed desirable binding properties to TCS. Therefore, these candidates were chosen for further analysis aiming at identifying the target binding site. Generally, it is accepted that the random region of the aptamer (N40 in this study) is the main contributor to aptamertarget interaction over iterative rounds of selection and the role of primer regions is of less importance (Bock et al. 1992; Marshall and Ellington 2000). Thus, for each aptamer, folding of the random region should ideally involve the primer regions as little as possible. As shown in Figure 3.8 (Chapter 3), folding of aptamer D5 showed a three-SL structure. The forward primer region (i.e nucleotides 1 to 18) folds into SL1, and removing this region should have no effect on the folding of the N40 region. Similarly, the reverse primer region (i.e. nucleotides 58 to 75 ) did not indicate any significant secondary structures, and removing this region should also not affecting the folding of the N40 region. In addition, the $\mathrm{N} 40$ region of the D5 composed of two SLs which are adjacent to each other by just one nucleotide. This is an interesting structure, as both primer regions are not involved in the folding with the N40 region. Thus, it is postulated that the target binding site of the aptamer D5 is potentially located in the N40 region, and either of the SL2 or the SL3, or both of them were responsible for the binding to TCS. 
In the secondary structure of aptamer E6 (Figure 3.8, Chapter 3), the reverse primer region (i.e nucleotides 58 to 75 ) interacted with part of the $\mathrm{N} 40$ region and formed the SL3, thus it was reasoned that removing this region should have a significant impact to the secondary structure of the N40 region. Whereas the nucleotide 18 in the forward primer region contributed to the formation of the SL2, thus removing the forward region should also have an impact on the N40 region (might be to some extent less than the reverse region). Overall, both primer regions were interacted with the $\mathrm{N} 40$ region of aptamer E6, and removing either regions should therefore affect its secondary structure. Once again, based on the assumption that the $\mathrm{N} 40$ region is the main contributor to the target binding site, either of SL2 or SL3 aptamer E6 should contribute to the binding to TCS.

In the case of TCS R10 aptamers (Figure 4.6, Chapter 4), the secondary structure of aptamer $\mathrm{H} 2$ showed that the forward primer region (i.e. nucleotides 1 to 18 ) interacted with part of the N40 region to form SL2, and the remaining N40 region as well as the reverse primer region did not fold into any significant secondary structures. Therefore, it is difficult to postulate where the target binding site would be located. The secondary structure of aptamer $\mathrm{H} 6$ is another interesting structure similar to the aptamer D5 mentioned before. As shown in Figure 4.6 (Chapter 4), only a couple of nucleotides (i.e. nucleotides 58 and 59) in the reverse primer region interacted with the N40 region, and the remaining reverse region as well as the forward region (i.e. nucleotides 1 to 18) had little interaction with the N40 region. Therefore, it can be postulated that the target binding site should be located in either SL2 or SL3 of the H6 aptamer, and also removal of either primer region should not significantly affect the secondary structure of this aptamer (see Appendix IX). The last secondary structure to be analysed was aptamer H5. As shown in Figure 4.6 (see Chapter 4), both the forward and reverse primer regions interacted with the N40 region and contributed to the formation of SL2 and SL4 respectively. The remaining N40 region also demonstrated a stem-loop structure (i.e. SL1). Thus, either individually or a combination of the three stem-loops, i.e. the SL1, SL2 and SL4, should perhaps be responsible for the binding to TCS. 
After identifying the regions of interest (i.e. stem-loops within each chosen aptamer), the next step of the structural analysis was to investigate whether those conserved sequences (i.e. "GGTTX", "XGGGX", "GGGGX", and "TGGGGGX") were involved in the formation of these regions. Since only the TCS R10 aptamers showed conserved sequences, the following analysis focused on the secondary structures of aptamers H2, H5 and H6 (Figure 4.6, Chapter 4). [i] The N40 region of aptamer H2 contained the conserved sequences "GGGGX", "XGGGX" and "TGGGGGX". Among them, the "GGGGX" and "XGGGX" contributed to the formation of SL2, whereas the "TGGGGGX" located region did not fold into any secondary structures. [ii] The N40 region of aptamer $\mathrm{H} 6$ contained the conserved sequences "XGGGX", "GGGGX" and "GGTTX". The first two sequences involved in the formation of SL3, whilst the last sequence contributed to the folding of SL2. [iii] The N40 region of aptamer H5 contained the conserved sequences of "GGTTX" which appeared twice, as well as "XGGGX" and "TGGGGGX". Among them, "GGTTX" contributed to the formation of SL1 and SL2, and the remaining sequences showed little effect on the formation of stem-loops.

In summary, the conserved sequences "XGGGX", "GGGGX" and "GGTTX" contributed to the formation of stem-loops (either alone or a combination) to the three best binding aptamers (H2, H5 and H6). Interesting, aptamer $\mathrm{H} 6 \mathrm{had}$ all of these conserved sequences, and also demonstrated the best binding capability to TCS $\left(K_{d}=378 \mathrm{nM}\right)$. Aptamers $\mathrm{H} 2$ containing two of the conserved sequences (i.e. "XGGGX" and "GGGGX") and H5 containing one of the conserved sequences (i.e. "GGTTX) but this appeared twice, also showed appreciable binding to TCS ( $K_{d}=1448$ and $584 \mathrm{nM}$ respectively). Therefore, it is safe to assume that these conserved sequences (either alone or a combination) could be at least part of the target binding site. The finding also suggested that aptamer H6 could be truncated by removing both of its primer regions without significantly altering the folding of the target binding site.

Overall, sequence alignment and secondary structure prediction revealed some conserved sequences that in principle might be associated with the target binding site. Therefore, it is reasonable to speculate that the aforementioned structural analytical process can assist in identifying regions of interest involved in aptamer binding to their molecular targets. However, further experimental work, such as those involving truncation of aptamers, is needed to validate this possibility. 


\subsection{Aptamer truncation study}

Aptamer truncation studies were performed with the aim of locating target binding domain, and to elucidate the role of primer and random regions for binding to the target molecule. Results from the truncation studies showed that the removal of both primer regions completely abolished the affinity of the H6 aptamer to TCS (Figure 4.11b, Chapter 4). Similarly, the binding affinity of the H6 aptamer was severly reduced after the removal of the forward primer region. In addition, truncation of the reverse primer region showed a reduced binding affinity to TCS by approximately two-fold than the parent aptamer (Figure 4.11b, Chapter 4). Therefore, it was concluded that both primer regions contributed to the binding of the H6 aptamer to TCS, and the role of the forward primer region was more significant than the reverse primer region.

These observations were unexpected, as the in silico characterisations described in Section 6.5 suggested that deletion of either primer region should have little effect on the target binding site, and thus the truncated aptamers should still retain their binding capability to the target molecule. Nevertheless, these results indicated that truncation by simply removing primer regions is not an appropriate approach for locating the target binding pocket, and a specific truncation strategy needs to be developed in order to confirm whether the identified conserved sequences are part of the target binding site.

\subsection{Aptasensor}

In this study, a TCS aptasensor utilising the non-specific absorptive property of ssDNA aptamer onto the surface of AuNPs was developed for detecting TCS in spiked-water samples. The major advantages of this aptasensor includes simple to use, rapid, label-free, and avoiding the use of expensive equipment. Therefore, in principle it is well suited for monitoring EDCs. During the development of the aptasensor, a few optimisation steps are necessary for the aptasensor to achieve high sensitivity, stability and reproducibility. These included: [i] the determination of salt tolerance of AuNPs, as a high salt concentration can improve the sensitivity, but may also decrease the stability and reproducibility of an aptasensor; [ii] the optimisation of the amount of aptamers required, 
as too much aptamers in the sensing system would sacrifice the sensitivity, whereas too little would decrease the stability and reproducibility and; [iii] the investigation of the aptamer target-induced aggregation, as some aptamer targets are charged and therefore can neutralise the charge on the surface of AuNPs, thereby inducing aggregation.

Initial studies demonstrated that the developed TCS aptasensor was capable of detecting TCS in the nanomolar range with evidence of weak non-specific interaction to structurally similar compounds (i.e. BPA and OE2). The non-specific interaction to MTS by the H6 aptamer compromised its specificity. However, given the fact that MTS is a metabolite of TCS, affinity towards either of the molecules is a useful indication for the presence of TCS or its derivatives. In addition, the TCS aptasensor demonstrated a visual LOD of $150 \mathrm{nM}$ : this is comparable to the $L O D$ of the OE2-aptasensor (i.e.100 nM by naked-eye) when using the full length 75 mer OE2-aptamer for protection, despite the fact that the H6 aptamer possessed a much smaller conformational change upon binding to TCS (Figure 4.9 D, E and F, Chapter 4) compared with the OE2-aptamer (Alsager et al. 2015). These results suggested that the $L O D$ of an aptasensor was not necessarily relied on an aptamer possessing a high level of conformational change, as similar results were also observed with a BPA aptasensor developed by Dr Shalen Kumar. In his study, the BPA aptasensor showed a visual $L O D$ of $200 \mathrm{nM}$, but the 75 mer BPA-aptamer used in the aptasensor did not show a significant conformational change upon target binding as well (Kumar 2014). Moreover, it is postulated that the $L O D$ of the TCS aptasensor could be improved further by subjecting the TCS aptamer H6 to specific truncations, thereby generating a smaller version of the aptamer which may dissociate from the surface of AuNPs more easily than the full sequence.

Furthermore, it is worth noting that most aptasensors, such as the ELISA-type, acoustic, electrochemical, and quantum dot-based aptasensors, were operated using specific buffering conditions which, in general, are similar to the SELEX buffers used for aptamer selection to ensure the formation of appropriate aptamer 3D structures. However, the aptasensor developed herein allows for the target validation without the dependency of a specific buffer system, thereby demonstrating the utility of the developed aptasensor for on-field sensing applications. 


\subsection{Future perspectives}

\subsubsection{Selection using a doped aptamer library}

The investigation of various selection strategies, such as negative and counter selections, have provided new insights into the critical steps in generating aptamers binding to small molecules. Utilising these strategies have also led to considerable improvement in the binding affinity of the selected aptamers. However, the $K_{d}$ values for the best binding aptamers generated are still at around $500 \mathrm{nM}$. Ideally these values should be below 100 $\mathrm{nM}$ to be considered as high affinity aptamers. To further improve aptamer binding affinity, it is suggested that future selection work could include a new selection strategy involving a partial randomised (doped) aptamer library. Since the currently selected aptamers have already shown some affinity and specificity to the target molecule, and based on their critical motifs that are responsible for binding with the target molecule, a doped aptamer library can be synthesised to reselect optimal aptamers: this can usually improve their affinity to the target. For example, high-affinity aptamers against HIV-1 aspartyl protease were obtained from a doped aptamer library based on the structural information of two previously obtained aptamers: in this instance binding affinities expressed as the $K_{d}$ values of the new aptamers, increased from 92-140 nM to 2-22 nM (Duclair et al. 2015). Therefore, using a dope aptamer library for selection, it is expected to improve the binding affinity of the current aptamers.

\subsubsection{Identify the target binding site}

In silico characterisation of the selected aptamers has enhanced our theoretical understanding of the evolutionary process in generating aptamers. The predicted aptamer secondary structures and the identified conserved sequences have demonstrated some possible locations that could be responsible for binding with the target molecule. However, there are no experimental data to confirm these regions are actually within the target binding sites, and the initial aptamer truncation studies did not provide good evidence to support this either. Therefore, the exact target binding pocket still needs to be discovered. 
General methods utilised for investigating aptamer-target binding sites include DNA footprinting (Zianni et al. 2006), x-ray crystallography (Ruigrok et al. 2012) and nuclear magnetic resonance (Kelly et al. 1996). However, these methods have been used mainly with aptamers interacting with proteins, and methods for aptamers binding to small molecules are limited. One possible approach to locate the target binding pocket is to use a novel enzymatic digestion assay developed by Dr Shalen Kumar. This method utilises the enzymatic activity of exonuclease to specifically cleave the unbound regions of a target-bound aptamer, and the method is dependent upon the capability of an aptamer to form a stable secondary structure once co-incubated with the target molecule (IP protected work). Experimental work towards the use of this novel method to identify the target binding site is ongoing.

\subsubsection{Further use of the developed aptasensor}

The development of an AuNP aptasensor for TCS detection is a significant achievement from this thesis. However, the developed aptasensor has only been tested in the targetspiked water samples. Therefore, testing the utility of the aptasensor in real samples, such as the environmental samples from wastewater treatment plants, or biological samples from animals or humans, is another important future perspective. Furthermore, as mentioned earlier the $L O D$ of the aptasensor does need to be improved to expand its versatility, and this can be achieved through the use of refined aptamers from the doped selection or shortened-aptamers by specific truncation. 


\section{LIST OF REFERENCES}

Ahn KC, Zhao B, Chen J, Cherednichenko G, Sanmarti E, Denison MS, Lasley B, Pessah IN, Kultz D, Chang DPY, Gee SJ and Hammock BD (2008). "In vitro biologic activities of the antimicrobials triclocarban, its analogs, and triclosan in bioassay screens: Receptor-based bioassay screens." Environmental Health Perspectives 116(9): 1203-1210.

Alsager OA, Kumar S, Zhu B, Travas-Sejdic J, McNatty KP and Hodgkiss JM (2015). "Ultrasensitive colorimetric detection of 17 beta-estradiol: the effect of shortening DNA aptamer sequences." Analytical Chemistry 87(8): 4201-4209.

Aldous E, Rockett L and Johnson I (2009). "Proposed EQS for Water Framework Directive Annex VIII substances: Triclosan (for consultation)." Water Framework Directive - United Kingdom Technical Advisory Group.

Amaral Mendes JJ (2002). "The endocrine disrupters: a major medical challenge." Food and chemical toxicology : An international journal published for the British Industrial Biological Research Association 40(6): 781-788.

Amaya-Gonzalez S, de-los-Santos-Alvarez N, Miranda-Ordieres AJ and Jesus LoboCastanon M (2013). "Aptamer-based analysis: a promising alternative for food safety control." Sensors 13(12): 16292-16311.

Aufartova J, Mahugo-Santana C, Sosa-Ferrera Z, Juan Santana-Rodriguez J, Novakova L and Solich P (2011). "Determination of steroid hormones in biological and environmental samples using green microextraction techniques: An overview." Analytica Chimica Acta 704(1-2): 33-46.

Avci-Adali M, Paul A, Wilhelm N, Ziemer G and Wendel HP (2010). "Upgrading SELEX technology by using lambda exonuclease digestion for single-stranded DNA generation." Molecules 15(1): 1-11.

Baaske P, Wienken CJ, Reineck P, Duhr S and Braun D (2010). "Optical thermophoresis for quantifying the buffer dependence of aptamer binding." Angewandte ChemieInternational Edition 49(12): 2238-2241.

Baker BR, Lai RY, Wood MS, Doctor EH, Heeger AJ and Plaxco KW (2006). "An electronic, aptamer-based small-molecule sensor for the rapid, label-free detection of cocaine in adulterated samples and biological fluids." Journal of the American Chemical Society 128(10): 3138-3139. 
Balagurumoorthy P, Brahmachari SK, Mohanty D, Bansal M and Sasisekharan V (1992). "Hairpin and parallel quartetstructures for telomeric sequences." Nucleic Acids Research 20(15): 4061-4067.

Barthelmebs L, Hayat A, Limiadi AW, Marty JL and Noguer T (2011). "Electrochemical DNA aptamer-based biosensor for OTA detection, using superparamagnetic nanoparticles." Sensors and Actuators B-Chemical 156(2): 932-937.

Bianchini M, Radrizzani M, Brocardo MG, Reyes GB, Solveyra CG and Santa-Coloma TA (2001). "Specific oligobodies against ERK-2 that recognize both the native and the denatured state of the protein." Journal of Immunological Methods 252(1-2): 191197.

Bing T, Chang TJ, Yang XJ, Mei, HC, Liu, XJ and Shangguan DH (2011). "G-quadruplex DNA aptamers generated for systemin." Bioorganic \& Medicinal Chemistry 19(14): 4211-4219.

Bock LC, Griffin LC, Latham JA, Vermaas EH and Toole JJ (1992). "Selection of singlestrand-DNA molecules that bind and inhibit human thrombin." Nature 355(6360): 564-566.

Brausch JM and Rand GM (2011). "A review of personal care products in the aquatic environment: Environmental concentrations and toxicity." Chemosphere 82(11): 1518-1532.

Bridonneau P, Chang YF, Buvoli VB, O'Connell D and Parma D (1999). "Site-directed selection of oligonucleotide antagonists by competitive elution." Antisense \& Nucleic Acid Drug Development 9(1): 1-11.

Brion F, Tyler CR, Palazzi X, Laillet B, Porcher JM, Garric J and Flammarion P (2004). "Impacts of 17 beta-estradiol, including environmentally relevant concentrations, on reproduction after exposure during embryo-larval-, juvenile- and adult-life stages in zebrafish (Danio rerio)." Aquatic Toxicology 68(3): 193-217.

Brun EM, Bonet E, Puchades R and Maquieira A (2008). "Selective enzyme-linked immunosorbent assay for triclosan. Application to wastewater treatment plant effluents." Environmental Science \& Technology 42(5): 1665-1672.

Bruno JG and Kiel JL (2002). "Use of magnetic beads in selection and detection of biotoxin aptamers by electrochemiluminescence and enzymatic methods." Biotechniques 32(1): 178-183. 
Calafat AM, Ye X, Wong LY, Reidy JA and Needham LL (2008). "Urinary concentrations of triclosan in the US population: 2003-2004." Environmental Health Perspectives 116(3): 303-307.

Campbell CG, Borglin SE, Green FB, Grayson A, Wozei E and Stringfellow WT (2006). "Biologically directed environmental monitoring, fate, and transport of estrogenic endocrine disrupting compounds in water: A review." Chemosphere 65(8): 12651280.

Cao Z, Tong R, Mishra A, Xu W, Wong GCL, Cheng J and Lu Y (2009). "Reversible cellSpecific drug delivery with aptamer-functionalized liposomes." Angewandte Chemie-International Edition 48(35): 6494-6498.

Carothers JM, Goler JA, Kapoor Y, Lara L and Keasling JD (2010). "Selecting RNA aptamers for synthetic biology: investigating magnesium dependence and predicting binding affinity." Nucleic Acids Research 38(8): 2736-2747.

Centi S, Tombelli S, Minunni M and Mascini M (2007). "Aptamer-based detection of plasma proteins by an electrochemical assay coupled to magnetic beads." Analytical Chemistry 79(4): 1466-1473.

Cha MJ, Lee HJ, Kim JS and Kim EY (2012). "Environmental assessment of estrogenic pollutants in Nam River of Korea using indirect competitive ELISA and e-screen assay." Toxicology and Environmental Health Sciences 4(4): 262-268.

Chan WCW, Maxwell DJ, Gao XH, Bailey RE, Han MY and Nie SM (2002). "Luminescent quantum dots for multiplexed biological detection and imaging." Current Opinion in Biotechnology 13(1): 40-46.

Chang CC and Huang SD (2010). "Determination of the steroid hormone levels in water samples by dispersive liquid-liquid microextraction with solidification of a floating organic drop followed by high-performance liquid chromatography." Analytica Chimica Acta 662(1): 39-43.

Chen J, Ahn KC, Gee NA, Gee SJ, Hammock BD and Lasley BL (2007). "Antiandrogenic properties of parabens and other phenolic containing small molecules in personal care products." Toxicology and Applied Pharmacology 221(3): 278-284.

Cheng PC (2006). "The contrast formation in optical microscopy." Springer US, Handbook of Biological Confocal Microscopy: 162-206. 
Cheng Y, Yong W, Marty JL and Xiurong Y (2011). "Aptamer-based colorimetric biosensing of Ochratoxin A using unmodified gold nanoparticles indicator." Biosensors \& Bioelectronics 26(5): 2724-2727.

Cho M, Oh SS, Nie J, Stewart R, Eisenstein M, Chambers J, Marth JD, Walker F, Thomson JA and Soh HT (2013). "Quantitative selection and parallel characterisation of aptamers." Proceedings of the National Academy of Sciences of the United States of America 110(46): 18460-18465.

Cho M, Xiao Y, Nie J, Stewart R, Csordas AT, Oh SS, Thomson JA and Soh HT (2010). "Quantitative selection of DNA aptamers through microfluidic selection and highthroughput sequencing." Proceedings of the National Academy of Sciences of the United States of America 107(35): 15373-15378.

Choi JH, Chen KH and Strano MS (2006). "Aptamer-capped nanocrystal quantum dots: A new method for label-free protein detection." Journal of the American Chemical Society 128(49): 15584-15585.

Choi JS, Kim SG, Lahousse M, Park HY, Park HC, Jeong B, Kim J, Kim SK and Yoon MY (2011). "Screening and characterisation of high-affinity ssDNA aptamers against anthrax protective antigen." Journal of Biomolecular Screening 16(2): 266-271.

Chu SG, Haffner GD and Letcher RJ (2005). "Simultaneous determination of tetrabromobisphenol A, tetrachlorobisphenol A, bisphenol $\mathrm{A}$ and other halogenated analogues in sediment and sludge by high performance liquid chromatography-electrospray tandem mass spectrometry." Journal of Chromatography A 1097(1-2): 25-32.

Ciesiolka J, Gorski J and Yarus M (1995). "Selection of an RNA domain that binds $\mathrm{Zn}^{2+}$." RNA-a Publication of the RNA Society 1(5): 538-550.

Commonwealth of Australia (2003). "National industrial chemicals notification and assessment scheme." Gazette Chemical (No. C5): 43-46.

Covaci A, Harrad S, Abdallah MAE, Ali N, Law RJ, Herzke D and de Wit CA (2011). "Novel brominated flame retardants: A review of their analysis, environmental fate and behaviour." Environment International 37(2): 532-556.

Crisp TM, Clegg ED, Cooper RL, Wood WP, Anderson DG, Baetcke KP, Hoffmann JL, Morrow MS, Rodier DJ, Schaeffer JE, Touart LW, Zeeman MG and Patel YM (1998). "Environmental endocrine disruption: An effects assessment and analysis." Environmental Health Perspectives 106: 11-56. 
Cruz-Aguado JA and Penner G (2008). "Determination of ochratoxin A with a DNA aptamer." Journal of Agricultural and Food Chemistry 56(22): 10456-10461.

Daniels DA, Chen H, Hicke BJ, Swiderek KM and Gold L (2003). "A tenascin-C aptamer identified by tumor cell SELEX: Systematic evolution of ligands by exponential enrichment." Proceedings of the National Academy of Sciences of the United States of America 100(26): 15416-15421.

Dapic V, Abdomerovic V, Marrington R, Peberdy J, Rodger A, Trent JO and Bates PJ (2003). "Biophysical and biological properties of quadruplex oligodeoxyribonucleotides." Nucleic Acids Research 31(8): 2097-2107.

Daughton CG (2004). "Non-regulated water contaminants: emerging research." Environmental Impact Assessment Review 24(7-8): 711-732.

Daughton CG and Ternes TA (1999). "Pharmaceuticals and personal care products in the environment: Agents of subtle change?" Environmental Health Perspectives 107: 907-938.

Davis JH and Szostak JW (2002). "Isolation of high-affinity GTP aptamers from partially structured RNA libraries." Proceedings of the National Academy of Sciences of the United States of America 99(18): 11616-11621.

Dayan AD (2007). "Risk assessment of triclosan [Irgasan ${ }^{\circledR}$ ] in human breast milk." Food and Chemical Toxicology 45(1): 125-129.

de Soultrait VR, Lozach PY, Altmeyer R, Tarrago-Litvak L, Litvak S and Andreola ML (2002). "DNA aptamers derived from HIV-1 RNase H inhibitors are strong antiintegrase agents." Journal of Molecular Biology 324(2): 195-203.

Deceuninck Y, Bichon E, Marchand P, Boquien CY, Legrand A, Boscher C, Antignac JP and Le Bizec B (2015). "Determination of bisphenol A and related substitutes/analogues in human breast milk using gas chromatography-tandem mass spectrometry." Analytical and Bioanalytical Chemistry 407(9): 2485-2497.

Di Primo C, Dausse E and Toulme JJ (2011). Surface plasmon resonance investigation of RNA aptamer-RNA ligand interactions. Therapeutic Oligonucleotides: Methods and Protocols. J. Goodchild. 764: 279-300.

Dias R, Mel'nikov S, Lindman B and Miguel MG (2000). "DNA phase behavior in the presence of oppositely charged surfactants." Langmuir 16(24): 9577-9583. 
Dias R, Rosa M, Pais AC, Miguel M and Lindman B (2004). "DNA-surfactant interactions, compaction, condensation, decompaction and phase separation." Journal of the Chinese Chemical Society 51(3): 447-469.

Dobbelstein M and Shenk T (1995). "In-vitro selection of RNA ligands for the ribosomall L22 protein associated with Epstein-Barr virus-expressed RNA by using randomised and cDNA-derived RNA libraries." Journal of Virology 69(12): 80278034.

Dominy BN (2008). "Molecular recognition and binding free energy calculations in drug development." Current Pharmaceutical Biotechnology 9(2): 87-95.

Drabovich A, Berezovski M and Krylov SN (2005). "Selection of smart aptamers by equilibrium capillary electrophoresis of equilibrium mixtures (ECEEM)." Journal of the American Chemical Society 127(32): 11224-11225.

Drolet DW, MoonMcDermott L and Romig TS (1996). "An enzyme-linked oligonucleotide assay." Nature Biotechnology 14(8): 1021-1025.

Duclair S, Gautam A, Ellington A and Prasad VR (2015). "High-affinity RNA aptamers against the HIV-1 protease inhibit both in vitro protease activity and late events of viral replication." Molecular Therapy-Nucleic Acids (4): 2162-2531.

Ebrahimi M (2007). "Vitellogenin assay by enzyme-linked immunosorbant assay as a biomarker of endocrine disruptor chemicals pollution." Pakistan journal of biological sciences 10(18): 3109-3114.

Eissa S, Ng A, Siaj M, Tavares AC and Zourob M (2013). "Selection and identification of DNA aptamers against okadaic acid for biosensing application." Analytical Chemistry 85(24): 11794-11801.

Elghanian R, Storhoff JJ, Mucic RC, Letsinger RL and Mirkin CA (1997). "Selective colorimetric detection of polynucleotides based on the distance-dependent optical properties of gold nanoparticles." Science 277(5329): 1078-1081.

Ellington AD and Szostak JW (1990). "Invitro selection pf RNA molecules that bind specific ligands." Nature 346(6287): 818-822.

Ellington AD and Szostak JW (1992). "Selection invitro of single strand-DNA molecules that fold specific ligand-binding structures." Nature 355(6363): 850-852.

Engvall E and Perlmann P (1971). "Enzyme-linked immunosorbent assay (ELISA) quantitative assay of immunoglobulin-G." Immunochemistry 8(9): 871-874. 
Eulberg D, Buchner K, Maasch C and Klussmann S (2005). "Development of an automated in vitro selection protocol to obtain RNA-based aptamers: identification of a biostable substance P antagonist." Nucleic Acids Research 33(4).

Eulberg D and Klussmann S (2003). "Spiegelmers: Biostable aptamers." Chembiochem 4(10): 979-983.

Fabrizio EF, Nadim A and Sterling JD (2003). "Resolution of multiple ssDNA structures in free solution electrophoresis." Analytical Chemistry 75(19): 5012-5021.

Farokhzad OC, Cheng JJ, Teply BA, Sherifi I, Jon S, Kantoff PW, Richie JP and Langer R (2006). "Targeted nanoparticle-aptamer bioconjugates for cancer chemotherapy in vivo." Proceedings of the National Academy of Sciences of the United States of America 103(16): 6315-6320.

Fasman GD (1996). "Circular Dichroism and the Conformational Analysis of Biomolecules." Plenum Press, New York, USA: 635-652

Feigon J, Dieckmann T and Smith FW (1996). "Aptamer structures from A to zeta." Chemistry \& Biology 3(8): 611-617.

Ferguson PL and Brownawell BJ (2003). "Degradation of nonylphenol ethoxylates in estuarine sediment under aerobic and anaerobic conditions." Environmental Toxicology and Chemistry 22(6): 1189-1199.

Fitter S and James R (2005). "Deconvolution of a complex target using DNA aptamers." Journal of Biological Chemistry 280(40): 34193-34201.

Foerster Ute, Weigand JE, Trojanowski P, Suess B and Wachtveitl J (2012). "Conformational dynamics of the tetracycline-binding aptamer." Nucleic Acids Research 40(4): 1807-1817.

Fredriksson S, Gullberg M, Jarvius J, Olsson C, Pietras K, Gustafsdottir SM, Ostman A and Landegren U (2002). "Protein detection using proximity-dependent DNA ligation assays." Nature Biotechnology 20(5): 473-477.

Freeman R, Girsh J and Willner I (2013). "Nucleic acid/quantum dots (QDs) hybrid systems for optical and photoelectrochemical sensing." ACS Applied Materials \& Interfaces 5(8): 2815-2834.

Fukusaki E, Hasunuma T, Kajiyama S, Okazawa A, Itoh TJ and Kobayashi A (2001). "SELEX for tubulin affords specific T-rich DNA aptamers." Bioorganic \& Medicinal Chemistry Letters 11(22): 2927-2930. 
Gafni J, Wong PW and Pessah IN (2004). "Non-coplanar 2,2 ',3,5 ',6-pentachlorobiphenyl (PCB 95) amplifies ionotropic glutamate receptor signaling in embryonic cerebellar granule neurons by a mechanism involving ryanodine receptors." Toxicological Sciences 77(1): 72-82.

Gebhardt K, Shokraei A, Babaie E and Lindqvist BH (2000). "RNA aptamers to Sadenosylhomocysteine: Kinetic properties, divalent cation dependency, and comparison with anti-S-adenosylhomocysteine antibody." Biochemistry 39(24): 7255-7265.

Geiger A, Burgstaller P, vonderEltz H, Roeder A and Famulok M (1996). "RNA aptamers that bind L-arginine with sub-micromolar dissociation constants and high enantioselectivity." Nucleic Acids Research 24(6): 1029-1036.

Gold L, Ayers D, Bertino J, Bock C, Bock A, Brody EN, Carter J, Dalby AB, Eaton BE, Fitzwater T, Flather D, Forbes A, Foreman T, Fowler C, Gawande B, Goss M, Gunn M, Gupta S, Halladay D, Heil J, Heilig J, Hicke B, Husar G, Janjic N, Jarvis T, Jennings S, Katilius E, Keeney TR, Kim N, Koch TH, Kraemer S, Kroiss L, Le N, Levine D, Lindsey W, Lollo B, Mayfield W, Mehan M, Mehler R, Nelson SK, Nelson M, Nieuwlandt D, Nikrad M, Ochsner U, Ostroff RM, Otis M, Parker T, Pietrasiewicz S, Resnicow DI, Rohloff J, Sanders G, Sattin S, Schneider D, Singer B, Stanton M, Sterkel A, Stewart A, Stratford S, Vaught JD, Vrkljan M, Walker JJ, Watrobka M, Waugh S, Weiss A, Wilcox SK, Wolfson A, Wolk SK, Zhang C and Zichi D (2010). "Aptamer-Based Multiplexed Proteomic Technology for Biomarker Discovery." Plos One 5(12).

Gomez JF, Brioso MA, Machado JD, Sanchez JL and Borges R (2002). New approaches for analysis of amperometrical recordings. Chromaffin Cell: Transmitter Biosynthesis, Storage, Release, Actions, and Informatics. D. T. Oconnor and L. E. Eiden. 971: 647654.

Gopinath SCB (2007). "Methods developed for SELEX." Analytical and Bioanalytical Chemistry 387(1): 171-182.

Gopinath SCB, Misono TS, Kawasaki K, Mizuno T, Imai M, Odagiri T and Kumar PKR (2006). "An RNA aptamer that distinguishes between closely related human influenza viruses and inhibits haemagglutinin-mediated membrane fusion." Journal of General Virology 87: 479-487. 
Grabar KC, Freeman RG, Hommer MB and Natan MJ (1995). "Preparation and characterisation of Au colloid monolayers." Analytical Chemistry 67(4): 735-743.

Gregoraszczuk EL, Rak A, Ludewig G and Gasinska A (2008). "Effects of estradiol, PCB3, and their hydroxylated metabolites on proliferation, cell cycle, and apoptosis of human breast cancer cells." Environmental Toxicology and Pharmacology 25(2): 227-233.

Halden RU and Paull DH (2005). "Co-occurrence of triclocarban and triclosan in US water resources." Environmental Science \& Technology 39(6): 1420-1426.

Hamula CLA, Guthrie JW, Zhang H, Li X, and Le XC (2006). "Selection and analytical applications of aptamers." Trac-Trends in Analytical Chemistry 25(7): 681-691.

Hayat A, Barthelmebs L, Sassolas A and Marty JL (2011). "An electrochemical immunosensor based on covalent immobilization of okadaic acid onto screen printed carbon electrode via diazotization-coupling reaction." Talanta 85(1): 513518.

Hayat A and Marty JL (2014). "Aptamer based electrochemical sensors for emerging environmental pollutants." Frontiers in chemistry 2: 41-41.

Hayat A, Yang C, Rhouati A and Marty JL (2013). "Recent advances and achievements in nanomaterial-based, and structure switchable aptasensing platforms for ochratoxin A Detection." Sensors 13(11): 15187-15208.

Henikoff S and Ahmad K (2005). Assembly of variant histones into chromatin. Annual Review of Cell and Developmental Biology. 21: 133-153.

Hermann T and Patel DJ (2000). "Biochemistry - Adaptive recognition by nucleic acid aptamers." Science 287(5454): 820-825.

Hianik T, Ostatna V, Sonlajtnerova M and Grman I (2007). "Influence of ionic strength, pH and aptamer configuration for binding affinity to thrombin." Bioelectrochemistry 70(1): 127-133.

Ho SP, Britton DHO, Stone BA, Behrens DL, Leffet LM, Hobbs FW, Miller JA and Trainor G L (1996). "Potent antisense oligonucleotides to the human multidrug resistance-1 mRNA are rationally selected by mapping RNA-accessible sites with oligonucleotide libraries." Nucleic Acids Research 24(10): 1901-1907.

Huang X, Li L, Qian H, Dong C and Ren J (2006). "A resonance energy transfer between chemiluminescent donors and luminescent quantum-dots as acceptors (CRET)." Angewandte Chemie-International Edition 45(31): 5140-5143. 
Huenniger T, Wessels H, Fischer C, Paschke-Kratzin A and Fischer M (2014). "Just in timeselection: A rapid semiautomated SELEX of DNA aptamers using magnetic separation and BEAMing." Analytical Chemistry 86(21): 10940-10947.

Huizenga DE, Sassanfar M and Szostak J (1993). "The invitro selection of ATP specific DNA aptamers." Journal of Cellular Biochemistry: 207-207.

Huizenga DE and Szostak JW (1995). "A DNA aptamer that binds adenosin and ATP." Biochemistry 34(2): 656-665.

Hutter HP, Wallner P, Hartl W, Uhl M, Lorbeer G, Gminski R, Mersch-Sundermann V and Kundi M (2010). "Higher blood concentrations of synthetic musks in women above fifty years than in younger women." International Journal of Hygiene and Environmental Health 213(2): 124-130.

Ireson CR and Kelland LR (2006). "Discovery and development of anticancer aptamers." Molecular Cancer Therapeutics 5(12): 2957-2962.

Jayasena SD (1999). "Aptamers: An emerging class of molecules that rival antibodies in diagnostics." Clinical Chemistry 45(9): 1628-1650.

Jenison RD, Gill SC, Pardi A and Polisky B (1994). "High-resolution molecular discrimination by RNA." Science 263(5152): 1425-1429.

Jo M, Ahn JY, Lee J, Lee S, Hong SW, Yoo JW, Kang J, Dua P, Lee D, Hong S and Kim S (2011). "Development of single-stranded DNA aptamers for specific bisphenol A detection." Oligonucleotides 21(2): 85-91.

Jobling S, Williams R, Johnson A, Taylor A, Gross-Sorokin M, Nolan M, Tyler CR, van Aerle R, Santos E and Brighty G (2006). "Predicted exposures to steroid estrogens in UK rivers correlate with widespread sexual disruption in wild fish populations." Environmental Health Perspectives 114: 32-39.

Joeng CB, Niazi JH, Lee SJ and Gu MB (2009). "ssDNA aptamers that recognize diclofenac and 2-anilinophenylacetic acid." Bioorganic \& Medicinal Chemistry 17(15): 53805387.

Kadioglu O, Malczyk AH, Greten HJ and Efferth T (2015). "Aptamers as a novel tool for diagnostics and therapy." Investigational New Drugs 33(2): 513-520.

Kang J, Lee MS and Gorenstein DG (2005). "The enhancement of PCR amplification of a random sequence DNA library by DMSO and betaine: Application to in vitro combinatorial selection of aptamers." Journal of Biochemical and Biophysical Methods 64(2): 147-151. 
Kannan K, Keith TL, Naylor CG, Staples CA, Snyder SA and Giesy JP (2003). "Nonylphenol and nonylphenol ethoxylates in fish, sediment, and water from the Kalamazoo River, Michigan." Archives of Environmental Contamination and Toxicology 44(1): 77-82.

Kearse M, Moir R, Wilson A, Stones-Havas S, Cheung M, Sturrock S, Buxton S, Cooper A, Markowitz S, Duran C, Thierer T, Ashton B, Meintjes P and Drummond A (2012). "Geneious Basic: An integrated and extendable desktop software platform for the organization and analysis of sequence data." Bioinformatics 28(12): 1647-1649.

Keefe AD, Pai S, Ellington A (2010). "Aptamers as therapeutics." Nature Reviews Drug Discovery 9(8): 537-550.

Kelly JA, Feigon J and Yeates TO (1996). "Reconciliation of the X-ray and NMR structures of the thrombin-binding aptamer d(GGTTGGTGTGGTTGG)." Journal of Molecular Biology 256(3): 417-422.

Kim JK, Choi KJ, Lee M, Jo M and Kim S (2012). "Molecular imaging of a cancer-targeting theragnostics probe using a nucleolin aptamer- and microRNA-221 molecular beacon-conjugated nanoparticle." Biomaterials 33(1): 207-217.

Kim YS and Gu MB (2014). Advances in aptamer screening and small molecule aptasensors. Biosensors Based on Aptamers and Enzymes. 140: 29-67.

Kim YS, Jung HS, Matsuura T, Lee HY, Kawai T and Gu MB (2007). "Electrochemical detection of 17 beta-estradiol using DNA aptamer immobilized gold electrode chip." Biosensors \& Bioelectronics 22(11): 2525-2531.

Kim YS, Kim JH, Kim IA, Lee SJ, Jurng J and Gu MB (2010). "A novel colorimetric aptasensor using gold nanoparticle for a highly sensitive and specific detection of oxytetracycline." Biosensors \& Bioelectronics 26(4): 1644-1649.

King SC and Hassell KL (2008). "Preliminaryassessment of an indirect ELISA and western blot techniques to screen vitellogenin in barramundi as a potential biomarker of exposure to EDCs in Australian tropical waters." Australasian Journal of Ecotoxicology 14(2-3, Sp. Iss. SI): 109-116.

Knop K, Hoogenboom R, Fischer D and Schubert US (2010). "Poly(ethylene glycol) in drug delivery: Pros and cons as well as potential alternatives." Angewandte ChemieInternational Edition 49(36): 6288-6308. 
Koh YKK, Lester JN and Scrimshaw MD (2005). "Fate and behavior of alkylphenols and their polyethoxylates in an activated sludge plant." Bulletin of Environmental Contamination and Toxicology 75(6): 1098-1106.

Koizumi M and Breaker RR (2000). "Molecular recognition of cAMP by an RNA aptamer." Biochemistry 39(30): 8983-8992.

Kuipers OP (1996). Random mutagenesis by using mixtures of dNTP and dITP in PCR. Methods in Molecular Biology; In vitro mutagenesis protocols. M. K. Trower. 57: 351-356.

Kumar A and Xagoraraki I (2010). "Pharmaceuticals, personal care products and endocrine-disrupting chemicals in U.S. surface and finished drinking waters: A proposed ranking system." Science of the Total Environment 408(23): 5972-5989.

Kumar S (2014). "Developing aptamers capable of binding oestradiol or bisphenol A." PhD Thesis, Victoria University of Wellington, New Zealand.

Kumar V, Balomajumder C and Roy P (2008). "Disruption of LH-induced testosterone biosynthesis in testicular Leydig cells by triclosan: Probable mechanism of action." Toxicology 250(2-3): 124-131.

Kumar V, Chakraborty A, Kural MR and Roy P (2009). "Alteration of testicular steroidogenesis and histopathology of reproductive system in male rats treated with triclosan." Reproductive Toxicology 27(2): 177-185.

Kwon M, Chun SM, Jeong S and Yu J (2001). "In vitro selection of RNA against kanamycin B." Molecules and Cells 11(3): 303-311.

Lao YH, Chiang HY, Yang DK, Peck K and Chen LC (2014). "Selection of aptamers targeting the sialic acid receptor of hemagglutinin by epitope-specific SELEX." Chemical Communications 50(63): 8719-8722.

Lao YH, Phua KKL and Leong KW (2015). "Aptamer nanomedicine for cancer therapeutics: Barriers and potential for translation." ACS Nanoletters 9(3): 2235-2254.

Larkin MA, Blackshields G, Brown NP, Chenna R, McGettigan PA, McWilliam H, Valentin F, Wallace IM, Wilm A, Lopez R, Thompson JD, Gibson TJ and Higgins DG (2007). "Clustal W and clustal X version 2.0." Bioinformatics 23(21): 2947-2948.

Larsson DGJ, Adolfsson-Erici M, Parkkonen J, Pettersson M, Berg AH, Olsson PE and Forlin L (1999). "Ethinyloestradiol - an undesired fish contraceptive?" Aquatic Toxicology 45(2-3): 91-97. 
Lato SM, Boles AR and Ellington AD (1995). "In-vitro selection of RNA lectins - using combinatorial chemistry to interpret ribozyme evolution." Chemistry \& Biology 2(5): 291-303.

Jeong S, Eom TY, Kim SJ, Lee SW and Yu J (2008). "ssDNA aptamer-based surface plasmon resonance biosensor for the detection of retinol binding protein 4 for the early diagnosis of type 2 diabetes." Analytical Chemistry 80(8): 2867-2873.

Legler J, Zeinstra LM, Schuitemaker F, Lanser PH, Bogerd J, Brouwer A, Vethaak AD, De Voogt P, Murk AJ and Van der Burg B (2002). "Comparison of in vivo and in vitro reporter gene assays for short-term screening of estrogenic activity." Environmental Science \& Technology 36(20): 4410-4415.

Leijs MM, Koppe JG, Olie K, van Aalderen WMC, de Voogt P and ten Tusscher GW (2009). "Effects of dioxins, PCBs, and PBDEs on immunology and hematology in adolescents." Environmental Science \& Technology 43(20): 7946-7951.

Lennarz S, Alich TC, Kelly T, Blind M, Beck H and Mayer G (2015). "Selective aptamerbased control of intraneuronal signaling." Angewandte Chemie-International Edition 54(18): 5369-5373.

Levy M, Cater SF and Ellington AD (2005). "Quantum-dot aptamer beacons for the detection of proteins." Chembiochem 6(12): 2163-2166.

Li F, Li J, Wang C, Zhang J, Li XF and Le XC (2011) "Competitive Protection of AptamerFunctionalized Gold Nanoparticles by Controlling the DNA Assembly." Analytical Chemistry 83(17): 6464-6467.

Li HX and Rothberg LJ (2004). "Label-free colorimetric detection of specific sequences in genomic DNA amplified by the polymerase chain reaction." Journal of the American Chemical Society 126(35): 10958-10961.

Li Y, Lee HJ and Corn RM (2007) "Detection of protein biomarkers using RNA aptamer microarrays and enzymatically amplified surface plasmon resonance imaging." Analytical Chemistry 79(3): 1082-1088.

Li YF, Geyer CR and Sen D (1996). "Recognition of anionic porphyrins by DNA aptamers." Biochemistry 35(21): 6911-6922.

Lin JS and McNatty KP (2009). "Aptamer-based regionally protected PCR for protein detection." Clinical Chemistry 55(9): 1686-1693. 
Liu JJ and Stormo GD (2005). "Combining SELEX with quantitative assays to rapidly obtain accurate models of protein-DNA interactions." Nucleic Acids Research $33(17)$.

Liu JW and Lu Y (2006). "Fast colorimetric sensing of adenosine and cocaine based on a general sensor design involving aptamers and nanoparticles." Angewandte Chemie-International Edition 45(1): 90-94.

Liu KC, Lin BS and Lan XP (2013). "Aptamers: A promising tool for cancer imaging, diagnosis, and therapy." Journal of Cellular Biochemistry 114(2): 250-255.

Liu XQ, Freeman R, Golub E and Willner I (2011). "Chemiluminescence and chemiluminescence resonance energy transfer (CRET) aptamer sensors using catalytic hemin/G-quadruplexes." ACS Nanoletters 5(9): 7648-7655.

Loyo-Rosales JE, Rice CP and Torrents A (2007). "Octyl and nonylphenol ethoxylates and carboxylates in wastewater and sediments by liquid chromatography/tandem mass spectrometry." Chemosphere 68(11): 2118-2127.

Lu J, Jin Q, He Y, Wu J, Zhang W and Zhao J (2008). "Anaerobic degradation behavior of nonylphenol polyethoxylates in sludge." Chemosphere 71(2): 345-351.

Luo XM, McKeague M, Pitre S, Dumontier M, Green J, Golshani A, Derosa MC AND Dehne F (2010). "Computational approaches toward the design of pools for the in vitro selection of complex aptamers." Rna-a Publication of the Rna Society 16(11): 2252-2262.

Ma HT, Liu JP, Ali MM, Mahmood MAI, Labanieh L, Lu MR, Iqbal SM, Zhang Q, Zhao WA and Wan Y (2015). "Nucleic acid aptamers in cancer research, diagnosis and therapy." Chemical Society Reviews 44(5): 1240-1256.

Mallikaratchy P, Tang ZW, Kwame S, Meng L, Shangguan DH and Tan WH (2007). "Aptamer directly evolved from live cells recognizes membrane bound immunoglobin heavy mu chain in Burkitt's lymphoma cells." Molecular \& Cellular Proteomics 6(12): 2230-2238.

Mann D, Reinemann C, Stoltenburg R and Strehlitz B (2005). "In vitro selection of DNA aptamers binding ethanolamine." Biochemical and Biophysical Research Communications 338(4): 1928-1934.

Mannironi C, DiNardo A, Fruscoloni P and TocchiniValentini GP (1997). "In vitro selection of dopamine RNA ligands." Biochemistry 36(32): 9726-9734. 
Marshall KA and Ellington AD (2000). "In vitro selection of RNA aptamers." Rna-Ligand Interactions, Part B 318: 193-214.

Martin JA, Phillips JA, Parekh P, Sefah K and Tan WH (2011). "Capturing cancer cells using aptamer-immobilized square capillary channels." Molecular Biosystems 7(5): 1720-1727.

Mascolo G, Locaputo V and Mininni G (2010). "New perspective on the determination of flame retardants in sewage sludge by using ultrahigh pressure liquid chromatography-tandem mass spectrometry with different ion sources." Journal of Chromatography A 1217(27): 4601-4611.

Maye MM, Han L, Kariuki NN, Ly NK, Chan WB, Luo J and Zhong CJ (2003). "Gold and alloy nanoparticles in solution and thin film assembly: Spectrophotometric determination of molar absorptivity." Analytica Chimica Acta 496(1-2): 17-27.

McKeague M, Bradley CR, De Girolamo A, Visconti A, Miller JD, DeRosa MC (2010). "Screening and initial binding assessment of fumonisin B-1 aptamers." International Journal of Molecular Sciences 11(12): 4864-4881.

Medintz IL, Uyeda HT, Goldman ER and Mattoussi H (2005). "Quantum dot bioconjugates for imaging, labelling and sensing." Nature Materials 4(6): 435-446.

Mehta J, Rouah-Martin E, Van Dorst B, Maes B, Herrebout W, Scippo ML, Dardenne F, Blust R and Robbens J (2012). "Selection and Characterisation of PCB-Binding DNA Aptamers." Analytical Chemistry 84(3): 1669-1676.

Mehta J, Van Dorst B, Rouah-Martin E, Herrebout W, Scippo ML, Blust R and Robbens J (2011). "In vitro selection and characterisation of DNA aptamers recognizing chloramphenicol." Journal of Biotechnology 155(4): 361-369.

Mei ZL, Chu HQ, Chen W, Xue F, Liu J, Xu HN, Zhang R and Zheng L (2013). "Ultrasensitive one-step rapid visual detection of bisphenol A in water samples by label-free aptasensor." Biosensors \& Bioelectronics 39(1): 26-30.

Mendonsa SD and Bowser MT (2004). "In vitro selection of high-affinity DNA ligands for human IgE using capillary electrophoresis." Analytical Chemistry 76(18): 53875392.

Mendonsa SD and Bowser MT (2005). "In vitro selection of aptamers with affinity for neuropeptide Y using capillary electrophoresis." Journal of the American Chemical Society 127(26): 9382-9383. 
Michaud M, Jourdan E, Villet A, Ravel A, Grosset C and Peyrin E (2003). "A DNA aptamer as a new target-specific chiral selector for HPLC." Journal of the American Chemical Society 125(28): 8672-8679.

Mills LJ and Chichester C (2005). "Review of evidence: Are endocrine-disrupting chemicals in the aquatic environment impacting fish populations?" Science of the Total Environment 343(1-3): 1-34.

Murphy MB, Fuller ST, Richardson PM and Doyle SA (2003). "An improved method for the in vitro evolution of aptamers and applications in protein detection and purification." Nucleic Acids Research 31(18).

Nakano M, Moody EM, Liang J and Bevilacqua PC (2002). "Selection for thermodynamically stable DNA tetraloops using temperature gradient gel electrophoresis reveals four motifs: d(cGNNAg), d(cGNABg), d(cCNNGg), and d(gCNNGc)." Biochemistry 41(48): 14281-14292.

Navani NK, Mok WK and Li YF (2009). In Vitro Selection of protein-binding DNA aptamers as ligands for biosensing applications. Methods in Molecular Biology. A. Rasooly and K. E. Herold. 504: 399-415.

Ng EWM, Shima DT, Calias P, Cunningham ET, Guyer DR and Adamis AP (2006). "Pegaptanib, a targeted anti-VEGF aptamer for ocular vascular disease." Nature Reviews Drug Discovery 5(2): 123-132.

Niazi JH, Lee SJ and Gu MB (2008). "Single-stranded DNA aptamers specific for antibiotics tetracyclines." Bioorganic \& Medicinal Chemistry 16(15): 7245-7253.

Nieto A, Borrull F, Maria MR and Pocurull E (2009). "Determination of personal care products in sewage sludge by pressurized liquid extraction and ultra high performance liquid chromatography-tandem mass spectrometry." Journal of Chromatography A 1216(30): 5619-5625.

Nieto A, Borrull F, Pocurull E and Marce RM (2008). "Determination of natural and synthetic estrogens and their conjugates in sewage sludge by pressurized liquid extraction and liquid chromatography-tandem mass spectrometry." Journal of Chromatography A 1213(2): 224-230.

Niu SC, Lv ZZ, Liu JC, Bai WH, Yang SM and Chen AL (2014). "Colorimetric aptasensor using unmodified gold nanoparticles for homogeneous multiplex detection." Plos One $9(10)$. 
Okazawa A, Maeda H, Fukusaki E, Katakura Y and Kobayashi A (2000). "In vitro selection of hematoporphyrin binding DNA aptamers." Bioorganic \& Medicinal Chemistry Letters 10(23): 2653-2656.

Ortiz de Garcia S, Pinto Pinto G, Garcia Encina P and Irusta Mata R (2013). "Consumption and occurrence of pharmaceutical and personal care products in the aquatic environment in Spain." Science of the Total Environment 444: 451-465.

Ostroff RM, Bigbee WL, Franklin W, Gold L, Mehan M, Miller YE, Pass HI, Rom WN, Siegfried JM, Stewart A, Walker JJ, Weissfeld JL, Williams S, Zichi D and Brody EN (2010). "Unlocking biomarker discovery: large scale application of aptamer proteomic technology for early detection of lung cancer." PloS one 5(12): e15003e15003.

Ouellet E, Foley JH, Conway EM and Haynes C (2015). "Hi-Fi SELEX: A high-fidelity digitalPCR based therapeutic aptamer discovery platform." Biotechnology and Bioengineering 112(8): 1506-1522.

Paborsky LR, McCurdy SN, Griffin LC, Toole JJ and Leung LLK (1993). "The singlestranded-DNA aptamer-binding site of human thrombin." Journal of Biological Chemistry 268(28): 20808-20811.

Paramasivan S, Rujan I and Bolton PH (2007). "Circular dichroism of quadruplex DNAs: Applications to structure, cation effects and ligand binding." Methods 43(4): 324331.

Pedrouzo M, Borrull F, Maria MR and Pocurull E (2009). "Ultra-high-performance liquid chromatography-tandem mass spectrometry for determining the presence of eleven personal care products in surface and wastewaters." Journal of Chromatography A 1216(42): 6994-7000.

Pedrouzo M, Borrull F, Maria Marce R and Pocurull E (2001). "National and regional assessment of the antibacterial soap market: A step toward determining the impact of prevalent antibacterial soaps." American Journal of Infection Control 29(5): 281-283.

Piganeau N and Schroeder R (2003). "Aptamer structures: A preview into regulatory pathways?" Chemistry \& Biology 10(2): 103-104.

Porath J and Fornsted N (1970). "Group fractionation of plasma proteins on dipolar ion exchangers. " Journal of Chromatography 51(3): 479-489. 
Proske D, Blank M, Buhmann R and Resch A (2005). "Aptamers - basic research, drug development, and clinical applications." Applied Microbiology and Biotechnology 69(4): 367-374.

Purdom CE, Hardiman PA, Bye VJ, Eno NC, Tyler CR and Sumpter JP (1994). "Estrogenic effects of effluents from sewage treatment works." Chemistry and Ecology 8(4): 275-285.

Qian JR, Lou XH, Zhang YT, Xiao Y, Soh HT (2009). "Generation of Highly Specific Aptamers via Micromagnetic Selection." Analytical Chemistry 81(13): 5490-5495.

Rhie A, Kirby L, Sayer N, Wellesley R, Disterer P, Sylvester I, Gill A, Hope J, James W and Tahiri-Alaoui A (2003). "Characterisation of 2 '-fluoro-RNA aptamers that bind preferentially to disease-associated conformations of prion protein and inhibit conversion." Journal of Biological Chemistry 278(41): 39697-39705.

Richardson SD and Ternes TA (2011). "Water analysis: Emerging contaminants and current issues." Analytical Chemistry 83(12): 4614-4648.

Romig TS, Bell C and Drolet DW (1999). "Aptamer affinity chromatography: combinatorial chemistry applied to protein purification." Journal of Chromatography B 731(2): 275-284.

Ruigrok VJB, Levisson M, Hekelaar J, Smidt H, Dijkstra BW and van der Oost J (2012). "Characterisation of aptamer-protein complexes by X-ray crystallography and alternative approaches." International Journal of Molecular Sciences 13(8): 10537-10552.

Rusconi CP, Scardino E, Layzer J, Pitoc GA, Ortel TL, Monroe D and Sullenger BA (2002). "RNA aptamers as reversible antagonists of coagulation factor IXa." Nature 419(6902): 90-94.

Saito K, Yagi K, Ishizaki A and Kataoka H (2010). "Determination of anabolic steroids in human urine by automated in-tube solid-phase microextraction coupled with liquid chromatography-mass spectrometry." Journal of Pharmaceutical and Biomedical Analysis 52(5): 727-733.

Saleh S, Haddadin RNS, Baillie S and Collier PJ (2010). “Triclosan - an update”. Letters in Applied Microbiology 52: 87-95.

Sandborgh-Englund G, Adolfsson-Erici M, Odham G and Ekstrand J (2006). "Pharmacokinetics of triclosan following oral ingestion in humans." Journal of Toxicology and Environmental Health-Part a-Current Issues 69(20): 1861-1873. 
Santosh B and Yadava PK (2014). "Nucleic acid aptamers: Research tools in disease diagnostics and therapeutics." Biomed Research International.

Schlensog MD, Gronewold TMA, Tewes M, Famulok M and Quandt E (2004). "Surface acoustic biosensors using aptamers as ligands." Springer-Verlag Berlin Heidelberg, Berlin. Functional Micro- and Nanosystems Chapter 9: 105-114.

Schoeneberg T, Hofreiter M, Schulz A and Roempler H (2007). "Learning from the past: evolution of GPCR functions." Trends in Pharmacological Sciences 28(3): 117-121.

Schwarzenbach RP, Escher BI, Fenner K, Hofstetter TB, Johnson CA, von Gunten U and Wehrli B (2006). "The challenge of micropollutants in aquatic systems." Science 313(5790): 1072-1077.

Sekiya S, Noda K, Nishikawa F, Yokoyama T, Kumar PKR and Nishikawa S (2006). "Characterisation and application of a novel RNA aptamer against the mouse prion protein." Journal of Biochemistry 139(3): 383-390.

Serganov A, Polonskaia A, Phan AT, Breaker RR and Patel DJ (2006). "Structural basis for gene regulation by a thiamine pyrophosphate-sensing riboswitch." Nature 441(7097): 1167-1171.

Shelver WL, Kamp LM, Church JL and Rubio FM (2007). "Measurement of triclosan in water using a magnetic particle enzyme immunoassay." Journal of Agricultural and Food Chemistry 55(10): 3758-3763.

Shigdar S, Lin J, Yu Y, Pastuovic M, Wei M and Duan W (2011). "RNA aptamer against a cancer stem cell marker epithelial cell adhesion molecule." Cancer Science 102(5): 991-998.

Silverman SK (2009). “Artificial functional nucleic acids: Aptamers, ribozymes, and deoxyribozymes identified by in vitro selection." Springer New York, Functional Nucleic Acids for Analytical Applications Part I: 47-108.

Singer H, Muller S, Tixier C and Pillonel L (2002). "Triclosan: Occurrence and fate of a widely used biocide in the aquatic environment: Field measurements in wastewater treatment plants, surface waters, and lake sediments." Environmental Science \& Technology 36(23): 4998-5004.

Song KM, Cho M, Jo HH, Min K, Jeon SH, Kim T, Han MS, Ku JK, Ban C (2011). "Gold nanoparticle-based colorimetric detection of kanamycin using a DNA aptamer." Analytical Biochemistry 415(2): 175-181. 
Song SP, Wang LH, Li J, Zhao JL, Fan CH (2008). "Aptamer-based biosensors." Trac-Trends in Analytical Chemistry 27(2): 108-117.

Staples C, Mihaich E, Carbone J, Woodburn K and Klecka G (2004). "A weight of evidence analysis of the chronic ecotoxicity of nonylphenol ethoxylates, nonylphenol ether carboxylates, and nonylphenol." Human and Ecological Risk Assessment 10(6): 999-1017.

Stojanovic MN, de Prada P and Landry DW (2001). "Aptamer-based folding fluorescent sensor for cocaine." Journal of the American Chemical Society 123(21): 4928-4931.

Stoltenburg R, Reinemann C and Strehlitz B (2005). "FluMag-SELEX as an advantageous method for DNA aptamer selection." Analytical and Bioanalytical Chemistry 383(1): 83-91.

Subedi B, Du BW, Chambliss CK, Koschorreck J, Ruedel H, Quack M, Brooks BW and Usenko S (2012). "Occurrence of pharmaceuticals and personal care products in german fish tissue: A national study." Environmental Science \& Technology 46(16): 9047-9054.

Sundberg L and Porath J (1974). "Preparation of adsorbents for biospecific affinity chromatography .1. attachment of group-containing ligands to insoluble polymers by means of bifunctional oxiranes." Journal of Chromatography 90(1): 87-98.

Takemura K, Wang P, Vorberg I, Surewicz W, Priola SA, Kanthasamy A, Pottathil R, Chen SG, Sreevatsan S (2006). "DNA aptamers that bind to PrPC and not PrPSc show sequence and structure specificity." Experimental Biology and Medicine 231(4): 485-485.

Tan Y, Shi YS, Wu XD, Liang HY, Gao YB, Li SJ, Zhang XM, Wang F and Gao TM (2013). "DNA aptamers that target human glioblastoma multiforme cells overexpressing epidermal growth factor receptor variant III in vitro." Acta Pharmacologica Sinica 34(12): 1491-1498.

Tang YT, Ge BX, Sen D, Yu HZ (2014). "Functional DNA switches: rational design and electrochemical signaling." Chemical Society Reviews 43(2): 518-529.

Tolle F, Wilke J, Wengel J and Mayer G (2014). "By-Product Formation in Repetitive PCR Amplification of DNA Libraries during SELEX." Plos One 9(12).

Tombelli S, Minunni A, Luzi E and Mascini M (2005). "Aptamer-based biosensors for the detection of HIV-1 Tat protein." Bioelectrochemistry 67(2): 135-141. 
Tombelli S, Minunni M, and Mascini M (2007). "Aptamers-based assays for diagnostics, environmental and food analysis." Biomolecular Engineering 24(2): 191-200.

Travascio P, Bennet AJ, Wang DY and Sen D (1999). "A ribozyme and a catalytic DNA with peroxidase activity: active sites versus cofactor-binding sites." Chemistry \& Biology 6(11): 779-787.

Travascio P, Li YF, Sen D (1998). "DNA-enhanced peroxidase activity of a DNA aptamerhemin complex." Chemistry \& Biology 5(9): 505-517.

Tsai RYL and Reed RR (1998). "Identification of DNA recognition sequences and protein interaction domains of the multiple-Zn-finger protein Roaz." Molecular and Cellular Biology 18(11): 6447-6456.

Tuerk C and Gold L (1990). "Systematic evolution of ligands by exponential enrichment RNA ligands to bacteriophage-T4 DNA-polymerase." Science 249(4968): 505-510.

Turner DJ, Tuytten R, Janssen KPF, Lammertyn J, Wuyts J, Pollet J, Eyckerman S, Brown C and Kas K (2011). "Toward clinical proteomics on a next-generation sequencing platform." Analytical Chemistry 83(3): 666-670.

Vega Morales T, Torres Padron ME, Sosa Ferrera Z, Santana Rodriguez JJ (2009). "Determination of alkylphenol ethoxylates and their degradation products in liquid and solid samples." Trac-Trends in Analytical Chemistry 28(10): 1186-1200.

Vivekananda J and Kiel JL (2006). "Anti-Francisella tularensis DNA aptamers detect tularemia antigen from different subspecies by aptamer-linked immobilized sorbent assay." Laboratory Investigation 86(6): 610-618.

Vonderheide AP (2009). "A review of the challenges in the chemical analysis of the polybrominated diphenyl ethers." Microchemical Journal 92(1): 49-57.

Vulliet E, Wiest L, Baudot R, Grenier-Loustalot MF (2008). "Multi-residue analysis of steroids at sub-ng/L levels in surface and ground-waters using liquid chromatography coupled to tandem mass spectrometry." Journal of Chromatography A 1210(1): 84-91.

Wallis MG, Streicher B, Wank H, vonAhsen U, Clodi E, Wallace ST, Famulok M and Schroeder R (1997). "In vitro selection of a viomycin-binding RNA pseudoknot." Chemistry \& Biology 4(5): 357-366.

Wang HQ, Wu Z, Tang LJ, Yu RQ, Jiang JH (2011). "Fluorescence protection assay: a novel homogeneous assay platform toward development of aptamer sensors for protein detection." Nucleic Acids Research 39(18). 
Wang J and Schnute WC (2010). "Direct analysis of trace level bisphenol A, octylphenols and nonylphenol in bottled water and leached from bottles by ultra-highperformance liquid chromatography/tandem mass spectrometry." Rapid Communications in Mass Spectrometry 24(17): 2605-2610.

Wang J, Wang LH, Liu XF, Liang ZQ, Song SP, Li WX, Li GX and Fan CH (2007). "A gold nanoparticle-based aptamer target binding readout for ATP assay." Advanced Materials 19(22): 3943-3946.

Wang J, Wang L, Liu XF, Liang ZQ, Song SP, Li WX, Li GX and Fan CH (2009). "Computational and experimental analyses converge to reveal a coherent yet malleable aptamer structure that controls chemical reactivity." Journal of the American Chemical Society 131(41): 14747-14755.

Wang XL, Wang XL, Su YH, Zhang C, Zhang LJ, Li F, Li BS, L XB, Xu SL, Li YY and Xu SQ (2005). "A high-sensitive new method to detect proteins based on DNA aptamers and exonuclease I." Zhongguo Shengwu Huaxue yu Fenzi Shengwu Xuebao 21(3): 421-426.

Wang ZW, Wu HB, Mao ZF, Hu XP, Zhang H, Hu ZP and Ren ZL (2014). "In vitro selection and identification of ssDNA aptamers recognizing the Ras protein." Molecular Medicine Reports 10(3): 1481-1488.

Waring RH and Harris RM (2005). "Endocrine disrupters: A human risk?" Molecular and Cellular Endocrinology 244(1-2): 2-9.

Wei H, Li BL, Li J, Wang E and Dong SJ (2007). "Simple and sensitive aptamer-based colorimetric sensing of protein using unmodified gold nanoparticle probes." Chemical Communications(36): 3735-3737.

Welch M, Majerfeld I and Yarus M (1997). "23S rRNA similarity from selection for peptidyl transferase mimicry." Biochemistry 36(22): 6614-6623.

White R, Rusconi C, Scardino E, Wolberg A, Lawson J, Hoffman M and Sullenger B (2001). "Generation of species cross-reactive aptamers using "toggle" SELEX." Molecular Therapy 4(6): 567-574.

Williams KP and Bartel DP (1995). "PCR products with strands of unequal length." Nucleic Acids Research 23(20): 4220-4221. 
Williams KP, Liu XH, Schumacher TNM, Lin HY, Ausiello DA, Kim PS and Bartel DP (1997). "Bioactive and nuclease-resistant L-DNA ligand of vasopressin." Proceedings of the National Academy of Sciences of the United States of America 94(21): 1128511290.

Wilson C and Szostak JW (1998). "Isolation of a fluorophore-specific DNA aptamer with weak redox activity." Chemistry \& Biology 5(11): 609-617.

Win MN, Klein JS and Smolke CD (2006). "Codeine-binding RNA aptamers and rapid determination of their binding constants using a direct coupling surface plasmon resonance assay." Nucleic Acids Research 34(19): 5670-5682.

Wong PW, Garcia EF and Pessah IN (2001). "ortho-Substituted PCB95 alters intracellular calcium signaling and causes cellular acidification in PC12 cells by an immunophilin-dependent mechanism." Journal of Neurochemistry 76(2): 450463.

Woo HM, Kim KS, Lee JM, Shim HS, Cho SJ, Lee WK, Ko HW, Keum YS, Kim SY, Pathinayake P, Kim CJ and Jeong YJ (2013). "Single-stranded DNA aptamer that specifically binds to the influenza virus NS1 protein suppresses interferon antagonism." Antiviral Research 100(2): 337-345.

Wu JJ, Zhu YY, Xue F, Mei ZL, Yao L, Wang X, Zheng L, Liu J, Liu GD, Peng CF and Chen W (2014). "Recent trends in SELEX technique and its application to food safety monitoring." Microchimica Acta 181(5-6): 479-491.

Wu ZS, Lu HX, Liu XP, Hu R, Zhou H, Shen GL and Yu RQ (2010). "Inhibitory effect of target binding on hairpin aptamer sticky-end pairing-induced gold nanoparticle assembly for light-up colorimetric protein assay." Analytical Chemistry 82(9): 3890-3898.

Xiang DX, Shigdar S, Qiao G, Wang T, Kouzani AZ, Zhou SF, Kong LX, Li Y, Pu CW and Duan W (2015). "Nucleic acid aptamer-guided cancer therapeutics and diagnostics: the next generation of cancer medicine." Theranostics 5(1): 23-42.

Xiang Y and Lu Y (2011). "Using personal glucose meters and functional DNA sensors to quantify a variety of analytical targets." Nature Chemistry 3(9): 697-703.

Xiao Y, Lubin AA, Heeger AJ, Plaxco KW (2005). "Label-free electronic detection of thrombin in blood serum by using an aptamer-based sensor." Angewandte Chemie-International Edition 44(34): 5456-5459. 
Xu SM, Yuan H, Chen SP, Xu A, Wang J and Wu LJ (2012). "Selection of DNA aptamers against polychlorinated biphenyls as potential biorecognition elements for environmental analysis." Analytical Biochemistry 423(2): 195-201.

Yan W, Li Y, Zhao LX and Lin JM (2009). "Determination of estrogens and bisphenol A in bovine milk by automated on-line $\mathrm{C}$-30 solid-phase extraction coupled with highperformance liquid chromatography-mass spectrometry." Journal of Chromatography A 1216(44): 7539-7545.

Yang XJ, Bing T, Mei HC, Fang CL, Cao ZH and Shangguan DH (2011). "Characterisation and application of a DNA aptamer binding to L-tryptophan." Analyst 136(3): 577585.

Yu-Ming C, Chen CKM and Ming-Hon H (2012). "Conformational changes in DNA upon ligand binding monitored by circular dichroism." Journal of Molecular Science (International Edition) 13(3): 3394-3413.

Yufa R, Krylova SM, Bruce C, Bagg EA, Schofield CJ, Krylov SN (2015). "Emulsion PCR significantly improves nonequilibrium capillary electrophoresis of equilibrium mixtures-based aptamer selection: Allowing for efficient and rapid selection of aptamer to unmodified ABH2 protein." Analytical Chemistry 87(2): 1411-1419.

Zeng SW, Baillargeat D, Ho HP and Yong KT (2014). "Nanomaterials enhanced surface plasmon resonance for biological and chemical sensing applications." Chemical Society Reviews 43(10): 3426-3452.

Zenker A, Schmutz H and Fent K (2008). "Simultaneous trace determination of nine organic UV-absorbing compounds (UV filters) in environmental samples." Journal of Chromatography A 1202(1): 64-74.

Zhang ZL, Shanmugam M and Rhind SM (2010). "PLE and GC-MS determination of polybrominated diphenyl ethers in soils." Chromatographia 72(5-6): 535-543.

Zhao RS, Wang X, Sun J, Wang SS, Yuan JP and Wang XK (2010). "Trace determination of triclosan and triclocarban in environmental water samples with ionic liquid dispersive liquid-phase microextraction prior to HPLC-ESI-MS-MS." Analytical and Bioanalytical Chemistry 397(4): 1627-1633.

Zhao WA, Chiuman W, Brook MA and Li YF (2007). "Simple and rapid colorimetric biosensors based on DNA aptamer and noncrosslinking gold nanoparticle aggregation." Chembiochem 8(7): 727-731. 
Zhou L, Ou LJ, Chu X, Shen GL and Yu RQ (2007). "Aptamer-based rolling circle amplification: A platform for electrochemical detection of protein." Analytical Chemistry 79(19): 7492-7500.

Zhou SN, Reiner EJ, Marvin C, Kolic T, Riddell N, Helm P, Dorman F, Misselwitz M and Brindle ID (2010). "Liquid chromatography-atmospheric pressure photoionization tandem mass spectrometry for analysis of 36 halogenated flame retardants in fish." Journal of Chromatography A 1217(5): 633-641.

Zianni M, Tessanne K, Merighi M, Laguna R and Tabita FR (2006). "Identification of the DNA bases of a DNase I footprint by the use of dye primer sequencing on an automated capillary DNA analysis instrument." Journal of Biomolecular Techniques 17(2): 103-113.

Zuker M (2003). "Mfold web server for nucleic acid folding and hybridisation prediction." Nucleic Acids Research 31(13): 3406-3415. 


\section{LIST OF APPENDICES}

\section{APPENDIX I}

Materials, reagents and instruments used in this study

1. Affinity matrix development

\begin{tabular}{|c|c|c|c|}
\hline Reagent/equipment & $\begin{array}{l}\text { Abbreviation/ } \\
\text { common name }\end{array}$ & $\begin{array}{l}\text { Catalogue } \\
\text { number/model }\end{array}$ & $\begin{array}{l}\text { Manufacturer/ } \\
\text { supplier }\end{array}$ \\
\hline $\begin{array}{l}\text { 5-Chloro-2-(2,4- } \\
\text { dichlorophenoxy)phen } \\
\text { ol }\end{array}$ & $\begin{array}{l}\text { Irgasan or } \\
\text { Triclosan (TCS) }\end{array}$ & PHR1338-1G & \multirow{7}{*}{$\begin{array}{l}\text { Sigma-Aldrich, St } \\
\text { Louis, Missouri, } \\
\text { USA }\end{array}$} \\
\hline $\begin{array}{l}\text { 1-Chloro-2,3- } \\
\text { epoxypropane }\end{array}$ & $\begin{array}{l}\text { Epichlorohydrin } \\
(\mathrm{ECH})\end{array}$ & E1055 & \\
\hline $\begin{array}{l}\text { 1,4-butanediol } \\
\text { diglycidyl ether }\end{array}$ & $\mathrm{DE}$ & 220892 & \\
\hline Sepharose ${ }^{\circledR} 6 \mathrm{~B}$ & & 6B100 & \\
\hline Sodium Chloride & $\mathrm{NaCl}$ & $7647-14-5$ & \\
\hline Sodium hydroxide & $\mathrm{NaOH}$ & $1310-73-2$ & \\
\hline $\begin{array}{l}\text { 2,2-Bis(4- } \\
\text { hydroxyphenyl) } \\
\text { propane }\end{array}$ & $\begin{array}{l}\text { Bisphenol A } \\
\text { (BPA) }\end{array}$ & 239658 & \\
\hline $\begin{array}{l}\text { 1, 3, 5(10)-Estratrien-3, } \\
17 \beta \text {-diol } 17 \text { - } \\
\text { hemisuccinate (C22 } \\
\text { H28 05) }\end{array}$ & $\begin{array}{l}\text { 17 } \beta \text {-oestradiol- } \\
17- \\
\text { hemisuccinate }\end{array}$ & E1076-000 & $\begin{array}{l}\text { Steraloids, } \\
\text { Newport, USA }\end{array}$ \\
\hline Hydrochloric Acid $36 \%$ & $\mathrm{HCl}$ & 1367 & $\begin{array}{l}\text { Thermo Fisher } \\
\text { Scientific, } \\
\text { Walthem, USA }\end{array}$ \\
\hline Syringe $(5 \mathrm{~mL})$ & Syringe & 302135 & $\begin{array}{l}\text { Becton, Dickinson } \\
\text { and }\end{array}$ \\
\hline Sintered plastic filters & Plastic filters & $\begin{array}{l}\text { Gifted by Dr Alan } \\
\text { Clark }\end{array}$ & $\begin{array}{l}\text { Sintered plastic } \\
\text { filters }\end{array}$ \\
\hline $\begin{array}{l}\text { UV-visible } \\
\text { spectrophotometer }\end{array}$ & UV-spec & Model: Cary 300 & $\begin{array}{l}\text { Agilent } \\
\text { Technologies, } \\
\text { Santa Clara, USA }\end{array}$ \\
\hline $\begin{array}{l}\text { Quartz SUPRASIL® } \\
\text { 1mm cuvettes }\end{array}$ & Quartz cuvettes & $\begin{array}{l}\text { 100-1-40 Type: } \\
100-Q S\end{array}$ & $\begin{array}{l}\text { Hellma }{ }^{\circledR} \\
\text { Analytics, } \\
\text { Müllheim, } \\
\text { Germany }\end{array}$ \\
\hline
\end{tabular}




\section{Synthetic oligonucleotides}

\begin{tabular}{|c|c|c|c|}
\hline Oligonucleotide & Sequence & Purification & Manufacturer \\
\hline $\begin{array}{l}\text { ssDNA N40 } \\
\text { library }\end{array}$ & $\begin{array}{l}\text { 5'-ATACGAGCTTGTTCAATA- } \\
\text { 40N-TGATAGTAAGAGCAAT } \\
\text { C-3' }\end{array}$ & \multirow[t]{3}{*}{ PAGE } & \multirow{6}{*}{$\begin{array}{l}\text { Life } \\
\text { Technologies, } \\
\text { Carlsbad, USA }\end{array}$} \\
\hline $\begin{array}{l}\text { Primer forward } \\
\text { (Pf40) }\end{array}$ & 5'-ATACGAGCTTGTTCAATA-3' & & \\
\hline $\begin{array}{l}\text { Primer reverse } \\
(\operatorname{Pr} 40)\end{array}$ & 5'-GATTGCTCTTACTATCA -3' & & \\
\hline $\begin{array}{l}\text { Biotin-Primer } \\
\text { forward (Pf40-B) }\end{array}$ & $\begin{array}{l}\text { 5'Biotin- } \\
\text { ATACGAGCTTGTTCAATA-3' }\end{array}$ & \multirow{2}{*}{ HPLC } & \\
\hline $\begin{array}{l}\text { Biotin-Primer } \\
\text { reverse (Pr40B) }\end{array}$ & $\begin{array}{l}\text { 5'Biotin-GATTGCTCTTACTATCA- } \\
\text { 3' }^{\prime}\end{array}$ & & \\
\hline $\begin{array}{l}\text { HEX-primer } \\
\text { forward (HEX-Pf) }\end{array}$ & 5'HEX-GATTGCTCTTACTATCA-3' & HPLC & \\
\hline
\end{tabular}

\section{Systemic Evolution of Ligands by Exponential Enrichment (SELEX)}

\begin{tabular}{|c|c|c|c|}
\hline Reagent/Equipment & $\begin{array}{l}\text { Abbreviation or } \\
\text { common name }\end{array}$ & $\begin{array}{l}\text { Catalogue } \\
\text { number/model }\end{array}$ & $\begin{array}{l}\text { Manufacturer } \\
\text { or supplier }\end{array}$ \\
\hline $\begin{array}{l}\text { Sodium Chloride } \\
\text { Reagent grade, purity } \\
99.5 \%\end{array}$ & $\mathrm{NaCl}$ & $7647-14-5$ & $\begin{array}{l}\text { Scharlau } \\
\text { Chemie, } \\
\text { Barcelona, Spain }\end{array}$ \\
\hline Calcium chloride & $\mathrm{CaCl} 2$ & 208291 & $\begin{array}{l}\text { Merck, } \\
\text { Kenilworth, USA }\end{array}$ \\
\hline $\begin{array}{l}\text { Tris(hydroxymethyl)- } \\
\text { aminomethane } \\
\text { Purity 99.9-100.1\% } \\
\text { (HOCH2)3CNH2 }\end{array}$ & Tris buffer & A1086 & $\begin{array}{l}\text { AppliChem, } \\
\text { Darmstadt } \\
\text { Germany }\end{array}$ \\
\hline Potassium Chloride & $\mathrm{KCl}$ & P9541 & \multirow{4}{*}{$\begin{array}{l}\text { Sigma-Aldrich, } \\
\text { St Louis, } \\
\text { Missouri, USA }\end{array}$} \\
\hline Magnesium Chloride & $\mathrm{MgCl} 2$ & M8266 & \\
\hline $\begin{array}{l}\text { Octylphenoxy } \\
\text { polyethoxyethanol or } \\
\text { Octylphenyl-polyethylene } \\
\text { glycol }\end{array}$ & $\begin{array}{l}\text { IGEPAL®CA-630 } \\
\text { or IGEPAL }\end{array}$ & $\begin{array}{l}9036-19-5 \\
\text { SKU\# I8896 }\end{array}$ & \\
\hline $\begin{array}{l}\text { Ribonucleic acid, transfer } \\
\text { from baker's yeast (S. } \\
\text { cerevisiae) }\end{array}$ & Yeast tRNA & R8759 & \\
\hline $0.1 \%$ fat Skim Milk powder & Skim milk & & $\begin{array}{l}\text { Fonterra } \\
\text { Auckland, New } \\
\text { Zealand }\end{array}$ \\
\hline IKA VXR basic Vibrax@ & Vibramix & 0002819000 & $\begin{array}{l}\text { IKA, Rawang, } \\
\text { Malaysia }\end{array}$ \\
\hline
\end{tabular}


4. Polymerase chain reaction and gel electrophoresis

\begin{tabular}{|c|c|c|c|}
\hline Reagent/Equipment & $\begin{array}{l}\text { Abbreviation/ } \\
\text { common name }\end{array}$ & $\begin{array}{l}\text { Catalogue } \\
\text { number/model }\end{array}$ & $\begin{array}{l}\text { Manufacturer/ } \\
\text { supplier }\end{array}$ \\
\hline $\begin{array}{l}\text { HotMaster }{ }^{\mathrm{TM}} \mathrm{Taq} \\
\text { polymerase kit }\end{array}$ & PCR kit & 2200310 & $\begin{array}{l}5 \text { Prime, Hamburg, } \\
\text { Germany }\end{array}$ \\
\hline $\begin{array}{l}\text { Nucleotides (dATP, dCTP, } \\
\text { dGTP and dTTP) }\end{array}$ & dNTPs & 10297-018 & \multirow{3}{*}{$\begin{array}{l}\text { Life Technologies, } \\
\text { Carlsbad, USA }\end{array}$} \\
\hline $\begin{array}{l}\text { SYBR Green I DNA staining } \\
\text { dye }\end{array}$ & SYBR green & S-7567 & \\
\hline $\begin{array}{l}\text { UltraPureTM distilled } \\
\text { water }\end{array}$ & $\begin{array}{l}\text { DNase free } \\
\text { water }\end{array}$ & 10977 & \\
\hline $\begin{array}{l}\text { Rotor-Gene Q 2plex HRM } \\
\text { Platform }\end{array}$ & $\begin{array}{l}\text { Real-time PCR } \\
\text { machine }\end{array}$ & 9001560 & \multirow[t]{2}{*}{$\begin{array}{l}\text { Qiagen, Hilden, } \\
\text { Germany }\end{array}$} \\
\hline Magnesium ions & $\mathrm{Mg}^{2+}$ ions & 201203 & \\
\hline $\begin{array}{l}\text { Quanta Biotech PCR } \\
\text { machine }\end{array}$ & PCR machine & Model QB 24 & $\begin{array}{l}\text { Quanta Biotech, } \\
\text { London, UK }\end{array}$ \\
\hline $\begin{array}{l}\text { Eppendorf Mastercycler® } \\
\text { Pro }\end{array}$ & PCR machine & $\begin{array}{l}\text { Mastercycler@ } \\
\text { Pro }\end{array}$ & $\begin{array}{l}\text { Eppendorf, } \\
\text { Hamburg, Germany }\end{array}$ \\
\hline SeaKem $®$ Agarose & Agarose gel & 50004 & $\begin{array}{l}\text { Lonza, Allendale, } \\
\text { New Jersey, USA }\end{array}$ \\
\hline $\begin{array}{l}\text { Horizontal electrophoresis } \\
\text { tank }\end{array}$ & Gel tank & $\begin{array}{l}\text { Sub-Cell } \\
\text { Model 96-170- } \\
4502\end{array}$ & $\begin{array}{l}\text { BioRad, Hercules, } \\
\text { California, USA }\end{array}$ \\
\hline 6X DNA loading dye & $\begin{array}{l}\text { DNA loading } \\
\text { dye }\end{array}$ & R0611 & \multirow{2}{*}{$\begin{array}{l}\text { Thermo Fisher } \\
\text { Scientific, } \\
\text { Walthem, USA }\end{array}$} \\
\hline Low range DNA ladder & DNA ladder & SM-1191 & \\
\hline $\begin{array}{l}\text { Ethylenediaminetetraaceti } \\
\text { c acid }\end{array}$ & EDTA & A1104 & $\begin{array}{l}\text { AppliChem. } \\
\text { Darmstadf, } \\
\text { Germany }\end{array}$ \\
\hline $\begin{array}{l}\text { 1,2,3-Propanetriol or } \\
\text { Glycerin }\end{array}$ & Glycerol & G5516 & $\begin{array}{l}\text { Sigma-Aldrich, St } \\
\text { Louis, Missouri, } \\
\text { USA }\end{array}$ \\
\hline
\end{tabular}

5. Gel extraction and strand separation

\begin{tabular}{|c|c|c|c|}
\hline Reagent/Equipment & $\begin{array}{l}\text { Abbreviation/ } \\
\text { common name }\end{array}$ & $\begin{array}{l}\text { Catalogue } \\
\text { number }\end{array}$ & $\begin{array}{l}\text { Manufacturer/ } \\
\text { supplier }\end{array}$ \\
\hline Qiagen Mini elute Kit & Gel extraction kit & 28006 & $\begin{array}{l}\text { Qiagen, Hilden, } \\
\text { Germany }\end{array}$ \\
\hline $\begin{array}{l}\text { Streptavidin coated } \\
\text { magnetic } \\
\text { beads }(4 \mathrm{mg} / \mathrm{mL})\end{array}$ & SMB & S1420S & $\begin{array}{l}\text { New England } \\
\text { BioLabs, Ipswich, } \\
\text { USA }\end{array}$ \\
\hline $\begin{array}{l}\text { PickPen } ® 1-\mathrm{M} \text { and } \\
\text { silicone tips }\end{array}$ & PickPen $®$ & 34096 & $\begin{array}{l}\text { Bio Noble, Zurich, } \\
\text { Switzerland }\end{array}$ \\
\hline
\end{tabular}




\section{Dot blotting experiments}

\begin{tabular}{|c|c|c|c|}
\hline Reagent/Equipment & $\begin{array}{l}\text { Abbreviation/ } \\
\text { common name }\end{array}$ & $\begin{array}{l}\text { Catalogue } \\
\text { number/model }\end{array}$ & $\begin{array}{l}\text { Manufacturer/ } \\
\text { supplier }\end{array}$ \\
\hline $\begin{array}{l}\text { HybondTM-C Extra } \\
\text { nitrocellulose } \\
\text { membrane }\end{array}$ & $\begin{array}{l}\text { Nitrocellulose } \\
\text { membrane }\end{array}$ & RPN303E & $\begin{array}{l}\text { Amersham GE } \\
\text { Biosciences, Little } \\
\text { Chalfont, UK }\end{array}$ \\
\hline $\begin{array}{l}\text { Strepavidin-Peroxidase } \\
\text { Polymer, Ultrasensitive }\end{array}$ & ST-HRP & S2438 & \multirow{4}{*}{$\begin{array}{l}\text { Sigma-Aldrich, St } \\
\text { Louis, Missouri, USA }\end{array}$} \\
\hline $\begin{array}{l}\text { Luminol } \\
\text { Assay } \geq 98 \% \text { (HPLC) }\end{array}$ & Luminol & C9008 & \\
\hline $\begin{array}{l}\text { 5-Chloro-2-(2,4- } \\
\text { dichlorophenoxy)anisol } \\
\text { e }\end{array}$ & Methyl triclosan & 34228 & \\
\hline $\begin{array}{l}\text { 2-[(2,6-Dichlorophenyl) } \\
\text { amino]benzeneacetic } \\
\text { acid sodium salt }\end{array}$ & $\begin{array}{l}\text { Diclofenac } \\
\text { sodium salt } \\
(\mathrm{DCF})\end{array}$ & D6899 & \\
\hline $\begin{array}{l}\text { p-Coumaric acid } \\
\geq 98.0 \% \text { (HPLC) }\end{array}$ & p-Coumaric acid & C9008 & $\begin{array}{l}\text { Scharlau Chemie, } \\
\text { Adelaide, Australia }\end{array}$ \\
\hline Kodak BioMax XAR Film & X-ray films & 1651454 & \multirow{4}{*}{$\begin{array}{l}\text { Kodak, Rochester, } \\
\text { New Jersey, USA }\end{array}$} \\
\hline Developing solution & Developer & 4037180 & \\
\hline Fixing solution & Fixer & 4037214 & \\
\hline Kodak X-Omatic cassette & X-ray cassette & KP 69040C & \\
\hline Stuart@ see-saw rocker & See-saw rocker & Model: SSL4 & Bibby Scientific Ltd, \\
\hline Lab Net Orbital LS & Orbital shaker & Orbital LS & Staffordshire, UK \\
\hline
\end{tabular}

\section{DNA ligation and transformation}

\begin{tabular}{|c|c|c|c|}
\hline Reagent/Equipment & $\begin{array}{l}\text { Abbreviation/ } \\
\text { common name }\end{array}$ & $\begin{array}{l}\text { Catalogue } \\
\text { number/model }\end{array}$ & $\begin{array}{l}\text { Manufacturer/ } \\
\text { supplier }\end{array}$ \\
\hline $\begin{array}{l}\text { pGEM®-T Easy Vector } \\
\text { Systems }\end{array}$ & Ligation kit & $\mathrm{A} 1360$ & $\begin{array}{l}\text { Promega, Madison, } \\
\text { Wisconsin, USA }\end{array}$ \\
\hline $\begin{array}{l}\text { Subcloning Efficiency } \\
\text { DH5 } 5 \alpha^{\mathrm{TM}} \text { Competent } \\
\text { Cells }\end{array}$ & $\begin{array}{l}\text { Competent E. coli } \\
\text { DH5- } \alpha \text { cells }\end{array}$ & 18265-017 & $\begin{array}{l}\text { Life Technologies, } \\
\text { Carlsbad, USA }\end{array}$ \\
\hline $\begin{array}{l}\text { Ampicillin anhydrous, } \\
96.0-100.5 \%\end{array}$ & Amp & $69-53-4$ & $\begin{array}{l}\text { Sigma-Aldrich, St } \\
\text { Louis, Missouri, USA }\end{array}$ \\
\hline BactoTM Yeast Extract & Yeast extract & 212750 & \multirow{3}{*}{$\begin{array}{l}\text { Becton, } \\
\text { Franklin, } \\
\text { Germany }\end{array}$} \\
\hline BactoTM Tryptone & Tryptone & 211705 & \\
\hline BactoTM Agar & Agar & 214010 & \\
\hline $\begin{array}{l}\text { EcoR I restriction } \\
\text { enzyme }\end{array}$ & $\begin{array}{l}\text { Restriction } \\
\text { enzyme }\end{array}$ & 10703737001 & \multirow{2}{*}{$\begin{array}{l}\text { RocheApplied } \\
\text { Science, } \\
\text { Basel, } \\
\text { Switzerland } \\
\end{array}$} \\
\hline $\begin{array}{l}\text { SuRE/Cut Buffer } \mathrm{H} \text { for } \\
\text { restriction digest }\end{array}$ & Buffer H & 11417991001 & \\
\hline $\begin{array}{l}\text { WPA Biowave II UV } \\
\text { spectrophotometer }\end{array}$ & UV spec & Biowave II & $\begin{array}{l}\text { Biochrom Ltd, } \\
\text { Cambridge, , UK }\end{array}$ \\
\hline
\end{tabular}




\section{Equipment used for aptamer characterisation}

\begin{tabular}{|l|l|l|l|}
\hline Equipment & Common name & Model & Manufacturer \\
\hline $\begin{array}{l}\text { ChirascanTM CD } \\
\text { Spectrometer }\end{array}$ & CD Spectrometer & TM-Plus & $\begin{array}{l}\text { Applied } \\
\text { Photophysics, } \\
\text { Cambridge, UK }\end{array}$ \\
\hline $\begin{array}{l}\text { UVsolo TS stand-alone gel } \\
\text { documentation system }\end{array}$ & Gel Doc System & TS stand-alone & $\begin{array}{l}\text { CORE Life Sciences, } \\
\text { Laguna Niguel, USA }\end{array}$ \\
\hline $\begin{array}{l}\text { Leica DM6000 FS fixed } \\
\text { stage fluorescent } \\
\text { microscope }\end{array}$ & $\begin{array}{l}\text { Fluorescent } \\
\text { microscope }\end{array}$ & DM-6000 FS & $\begin{array}{l}\text { Leica Microsystems, } \\
\text { Buffalo, USA }\end{array}$ \\
\hline
\end{tabular}

\section{Synthesis of gold nanoparticles (AuNPs)}

\begin{tabular}{|l|l|l|l|}
\hline Reagent/Equipment & $\begin{array}{l}\text { Abbreviation/ } \\
\text { common name }\end{array}$ & $\begin{array}{l}\text { Catalogue } \\
\text { number/model }\end{array}$ & $\begin{array}{l}\text { Manufacturer/ } \\
\text { supplier }\end{array}$ \\
\hline $\begin{array}{l}\text { Hydrogen tetrachloroaurate } \\
\text { hydrate }\end{array}$ & Gold chloride & 254169 & $\begin{array}{l}\text { Sigma-Aldrich, St } \\
\text { Louis, Missouri, } \\
\text { USA }\end{array}$ \\
\hline $\begin{array}{l}\text { Sodium citrate tribasic } \\
\text { dihydrate }\end{array}$ & Sodium citrate & 1613859 & $\begin{array}{l}\text { JEOL Solutions } \\
\text { Peabody, USA }\end{array}$ \\
\hline $\begin{array}{l}\text { JEOL USA Transmission } \\
\text { Electron Microscope }\end{array}$ & TEM & & $\begin{array}{l}\text { Agilent } \\
\text { Technologies, } \\
\text { Santa Clara, USA }\end{array}$ \\
\hline $\begin{array}{l}\text { Agilent 8453 UV-visible } \\
\text { spectrophotometer }\end{array}$ & UV-visible spec & 8453 & \\
\hline
\end{tabular}

\section{General solvents}

\begin{tabular}{|l|l|l|l|}
\hline Reagent & $\begin{array}{l}\text { Abbreviation or } \\
\text { common name }\end{array}$ & $\begin{array}{l}\text { Catalogue } \\
\text { number }\end{array}$ & $\begin{array}{l}\text { Manufacturer or } \\
\text { supplier }\end{array}$ \\
\hline $\begin{array}{l}\text { Absolute ethanol } \\
\text { (Analytical grade) }\end{array}$ & Ethanol (EtOH) & ET0016 & $\begin{array}{l}\text { Scharlau Chemie, } \\
\text { Adelaide, Australia }\end{array}$ \\
\hline $\begin{array}{l}\text { Propan-2-ol } \\
\text { (Analytical grade) }\end{array}$ & Iso propanol & AL0323 & \\
\hline
\end{tabular}

\section{General equipment}

\begin{tabular}{|l|l|l|l|}
\hline Equipment & common name & Model & $\begin{array}{l}\text { Manufacturer or } \\
\text { supplier }\end{array}$ \\
\hline $\begin{array}{l}\text { Still for HQ-distilled } \\
\text { water }\end{array}$ & $\begin{array}{l}\text { Water purifier } \\
\text { system }\end{array}$ & High-Q 103S & $\begin{array}{l}\text { High-Q, Wilmette, } \\
\text { USA }\end{array}$ \\
\hline Autoclave & & Systec DX 65 & $\begin{array}{l}\text { Systec, Wattenberg, } \\
\text { Germany }\end{array}$ \\
\hline $\begin{array}{l}\text { Sanyo Biomedical } \\
\text { Incubator }\end{array}$ & Incubator & MCO-5AC & $\begin{array}{l}\text { Sanyo/Panasonic, } \\
\text { Moriguchi, Japan }\end{array}$ \\
\hline
\end{tabular}




\section{APPENDIX II}

Secondary structures of triclosan round 8 aptamers D1 (TR8D1), D2 (TR8D2), D4 (TR8D4), D6 (TR8D6), E1 (TR8E1), E2 (TR8E2), E2 (TR8E3) and E5 (TR8E5) from the basic-SELEX procedure. Three aptamers (i.e. D1, E2, and E3) were folded into linear stemloop structures. Two aptamers (i.e. D6 and E5) showed two stem-loop structures, another two aptamers (i.e. D2 and E1) showed three stem-loop structures, and aptamer D4 showed a four stem-loop structure.

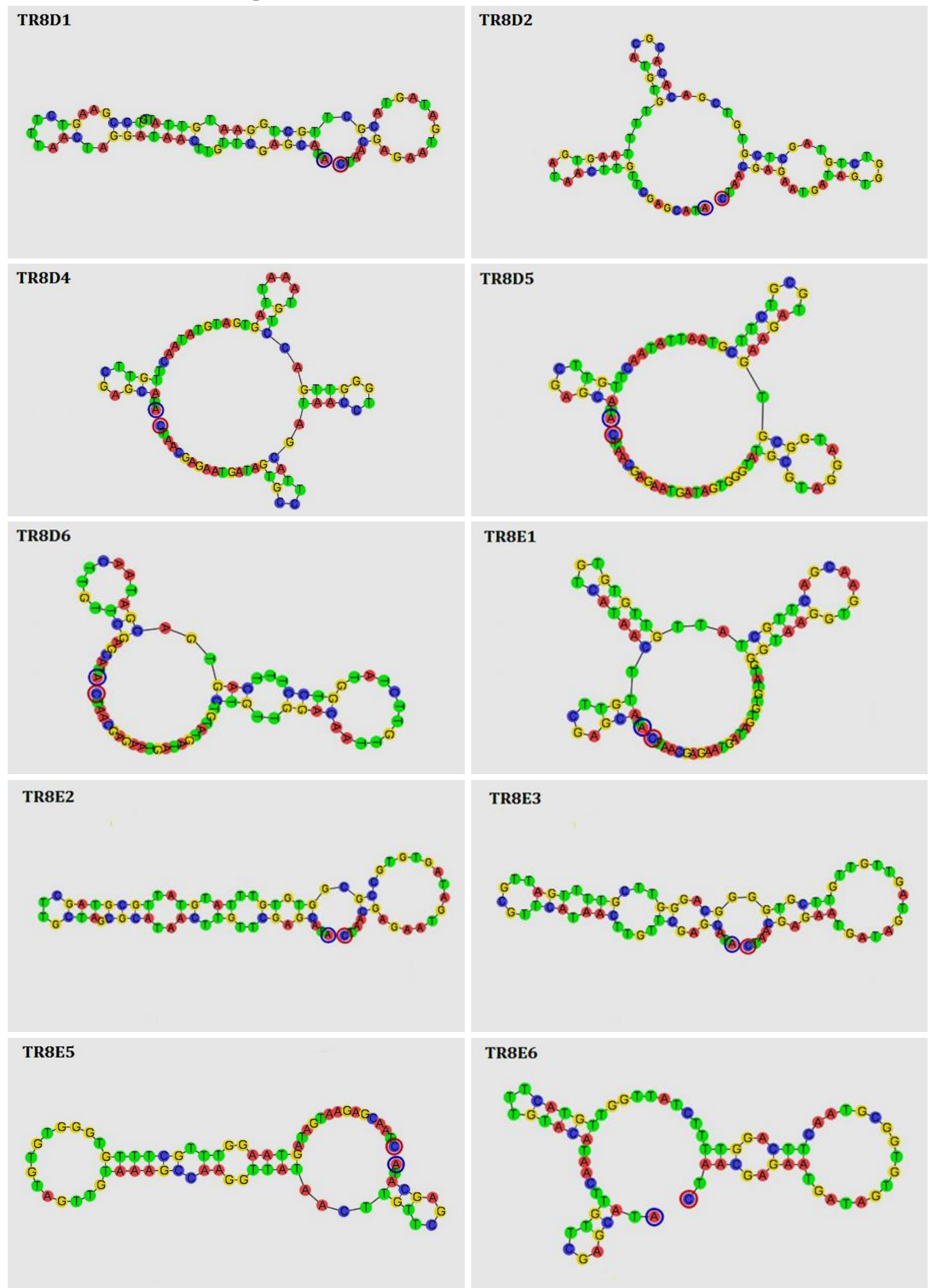




\section{APPENDIX III}

Control experiments for the affinity screening of TCS round 8 aptamers (i.e. D and E clones). The concentration range of the positive control NHS-biotin spots was from 16 to 2000 pmol: this was used to assess the consistency of the dot blot assays. The concentration of the negative control triclosan spots was at $600 \mathrm{nmol}$. This was used to assess non-specific interactions between triclosan and streptavidin-horse radish peroxidase.

\begin{tabular}{|c|c|c|c|c|c|c|}
\hline \multirow{2}{*}{$\begin{array}{r}(+\mathrm{ve}) \\
\text { control }\end{array}$} & \multicolumn{5}{|c|}{ NHS-biotin dot blots (pmol) } & \multirow{2}{*}{$\begin{array}{l}\text { (-ve) } \\
\text { control }\end{array}$} \\
\hline & 2000 & 400 & 80 & 16 & 0 & \\
\hline D1 and E1 & & & & & & \\
\hline D2 and E2 & & & & & & \\
\hline D4 and E3 & & & ค & ค & & \\
\hline D5 and E5 & & & ? & 0 & & \\
\hline D6 and E6 & & & & & & \\
\hline TCS & - & - & - & - & - & + \\
\hline Aptamer & - & - & - & - & - & - \\
\hline ST-HRP & + & + & + & + & + & + \\
\hline
\end{tabular}

TCS = triclosan; NHS = N-hydroxysuccinimide; ST-HRP = streptavidin-horseradish peroxidase; "+" means the presence of and; "-" means not present. 


\section{APPENDIX IV}

Secondary structures of triclosan round 10 aptamers H1 (TR10H1), H3 (TR10H3), H4 (TR10H4), H7 (TR10H7), H8 (TR10H8), H9 (TR10H9), and H10 (TR10H10) from the revised-SELEX procedure. Two variants ( $\mathrm{H} 7$ and $\mathrm{H} 9$ ) were folded into similar linear hairpin loop-stem structures. Another two variants (H4 and H10) displayed two stemloop structures. Aptamers H1 H3, and H8 displayed three stem-loop structures.

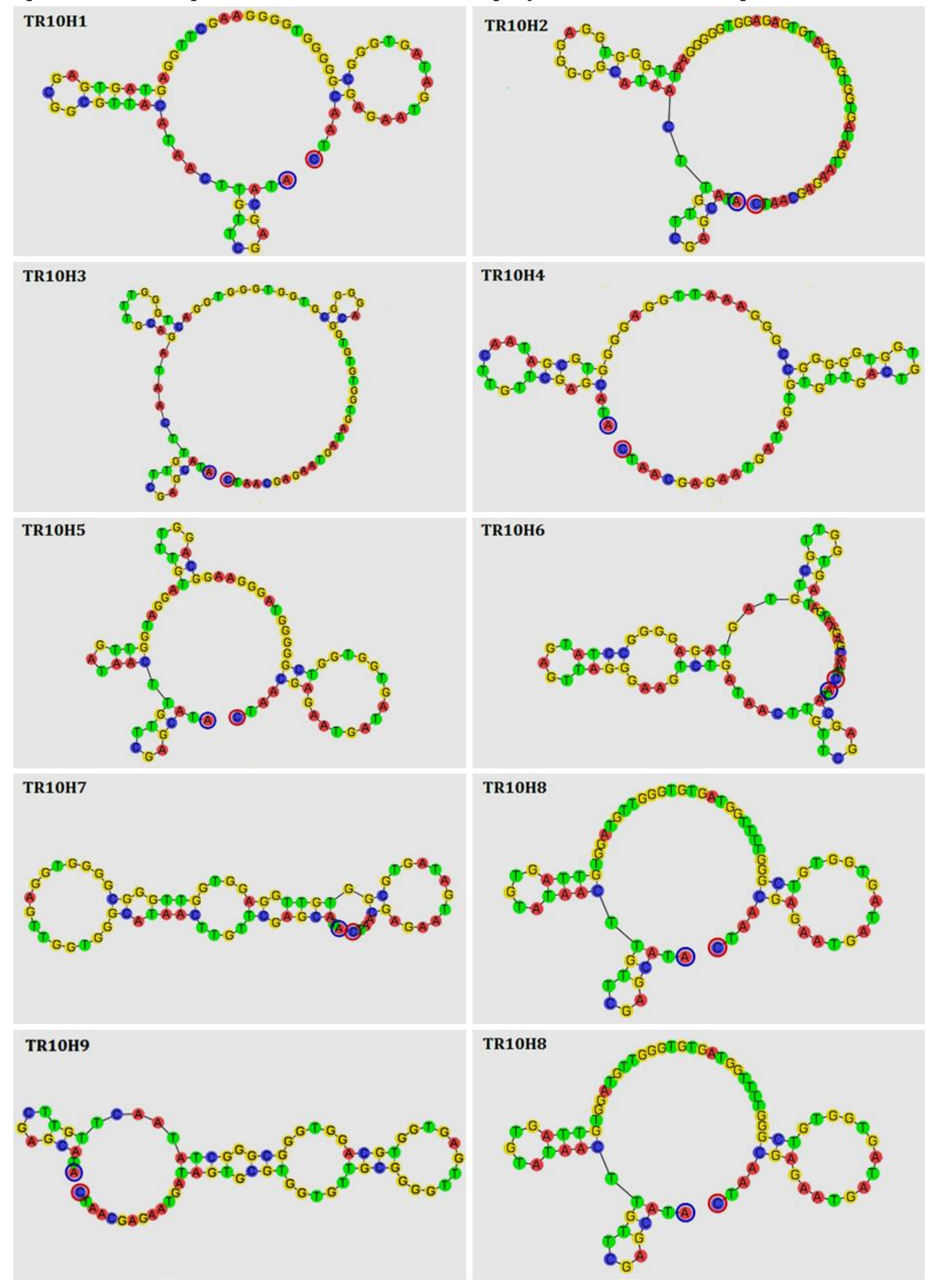




\section{APPENDIX V}

Fluorescent images of the HEX-labelled aptamers (i) H2, (ii) H5 and (iii) H6, as well as (iv) the random sequence (Rand) binding to control matrix at $750 \mathrm{nM}$ and binding to triclosan (TCS) conjugated matrix at 6 concentrations ranging from $20 \mathrm{nM}$ to $750 \mathrm{nM}$. A to E represents 5 independent repeats of the same experiment.

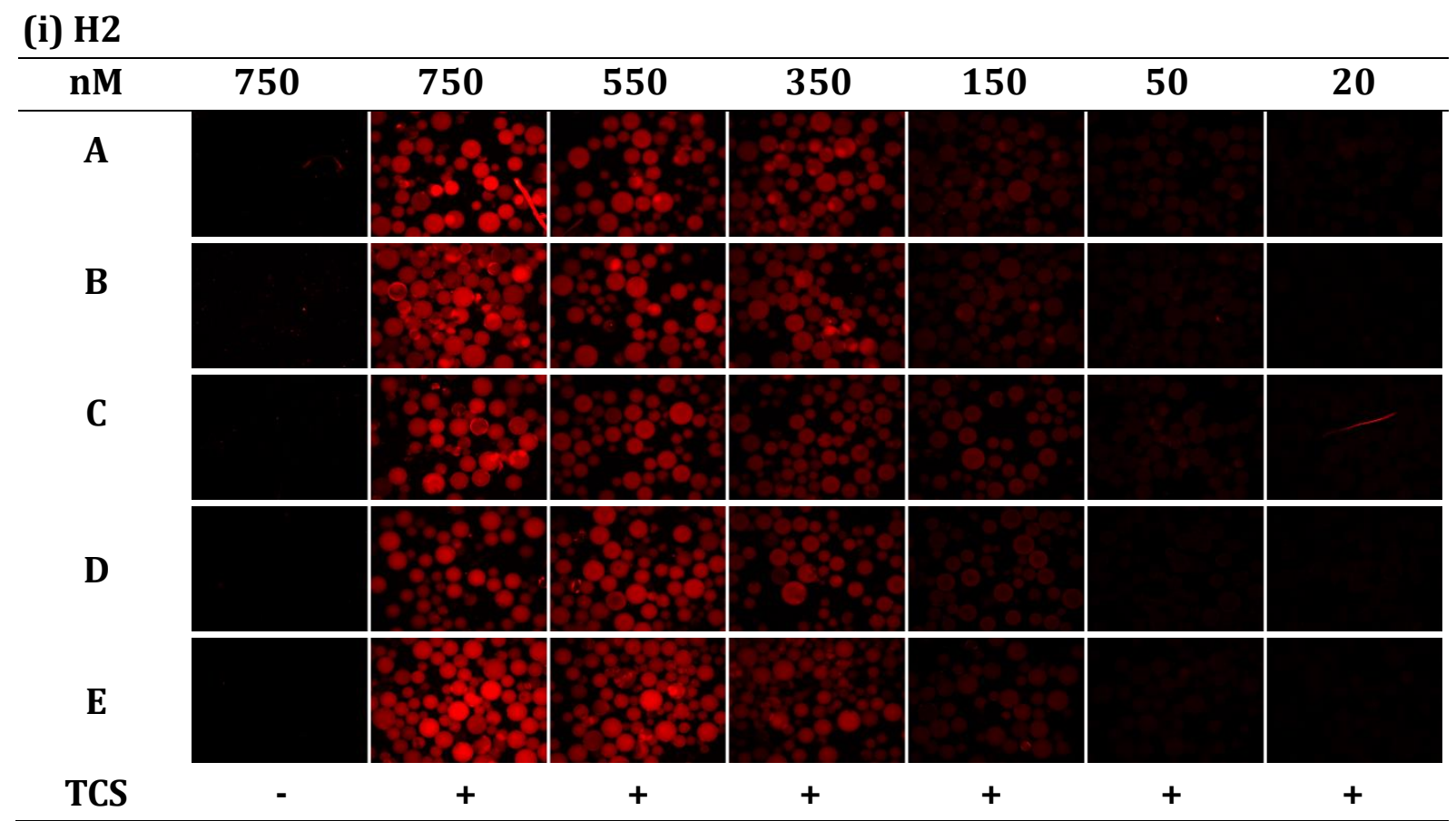

\begin{tabular}{|c|c|c|c|c|c|c|c|}
\hline \multicolumn{8}{|c|}{ (ii) H5 } \\
\hline $\mathbf{n M}$ & 750 & 750 & 550 & 350 & 150 & 50 & 20 \\
\hline \multicolumn{8}{|l|}{$\mathbf{A}$} \\
\hline \multicolumn{8}{|l|}{ B } \\
\hline \multicolumn{8}{|l|}{ C } \\
\hline \multicolumn{8}{|l|}{ D } \\
\hline \multicolumn{8}{|l|}{$\mathbf{E}$} \\
\hline TCS & - & + & + & + & + & + & + \\
\hline
\end{tabular}


(iii) $\mathrm{H6}$

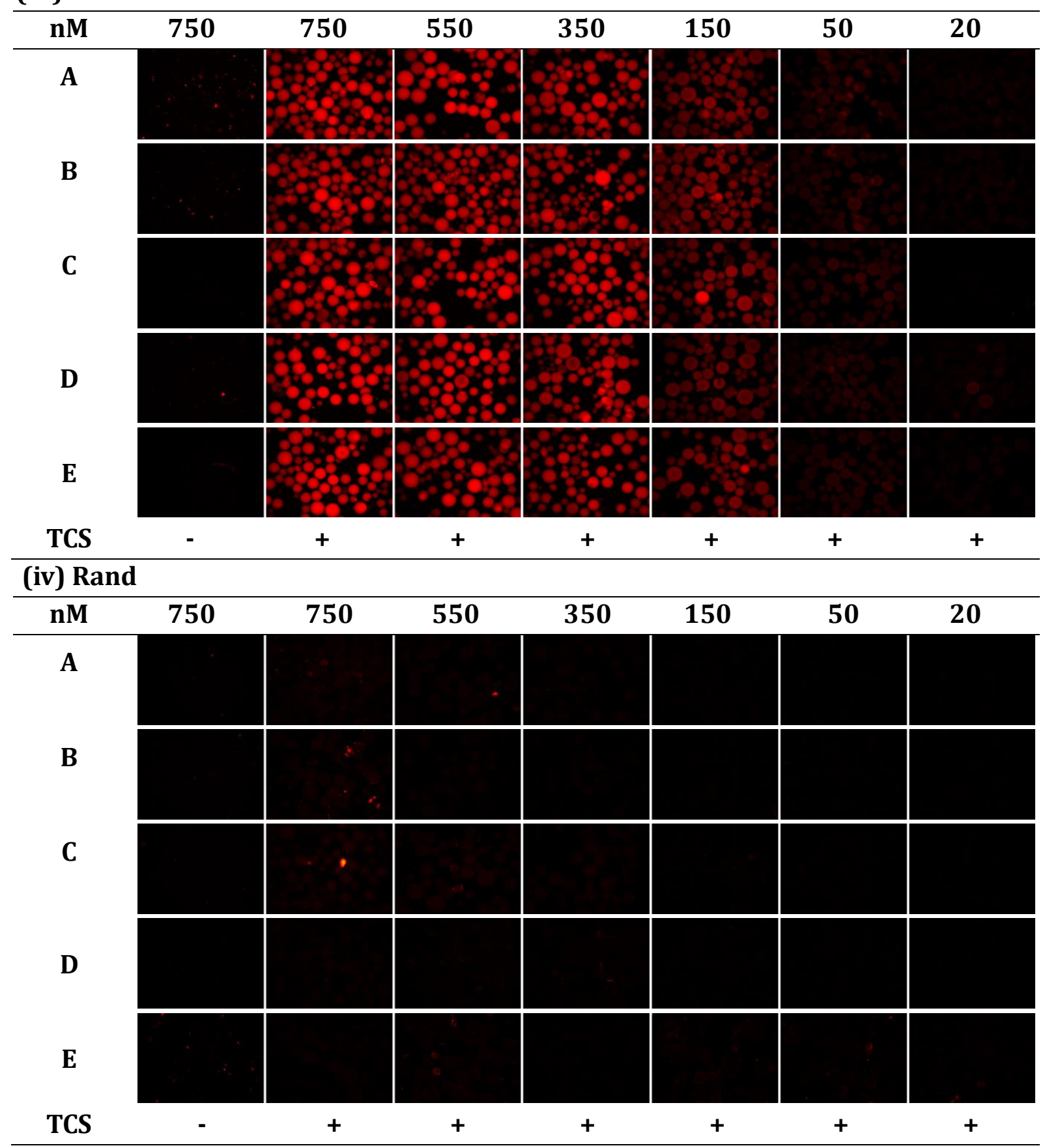

Each image was captured under the same conditions with 1 sec of exposure; "-" = control matrix and "+" = TCS-conjugated matrix. 


\section{APPENDIX VI}

Image analysis of the HEX-labelled aptamers (i) H2, (ii) $\mathrm{H} 5$ and (iii) H6, as well as (iv) the random sequence (Rand) binding to triclosan (TCS) conjugated matrix at 6 concentrations ranging from 20 to $750 \mathrm{nM}$. A to D represent fluorescent image, black and white image, selection of bead regions and selection of background regions, respectively.

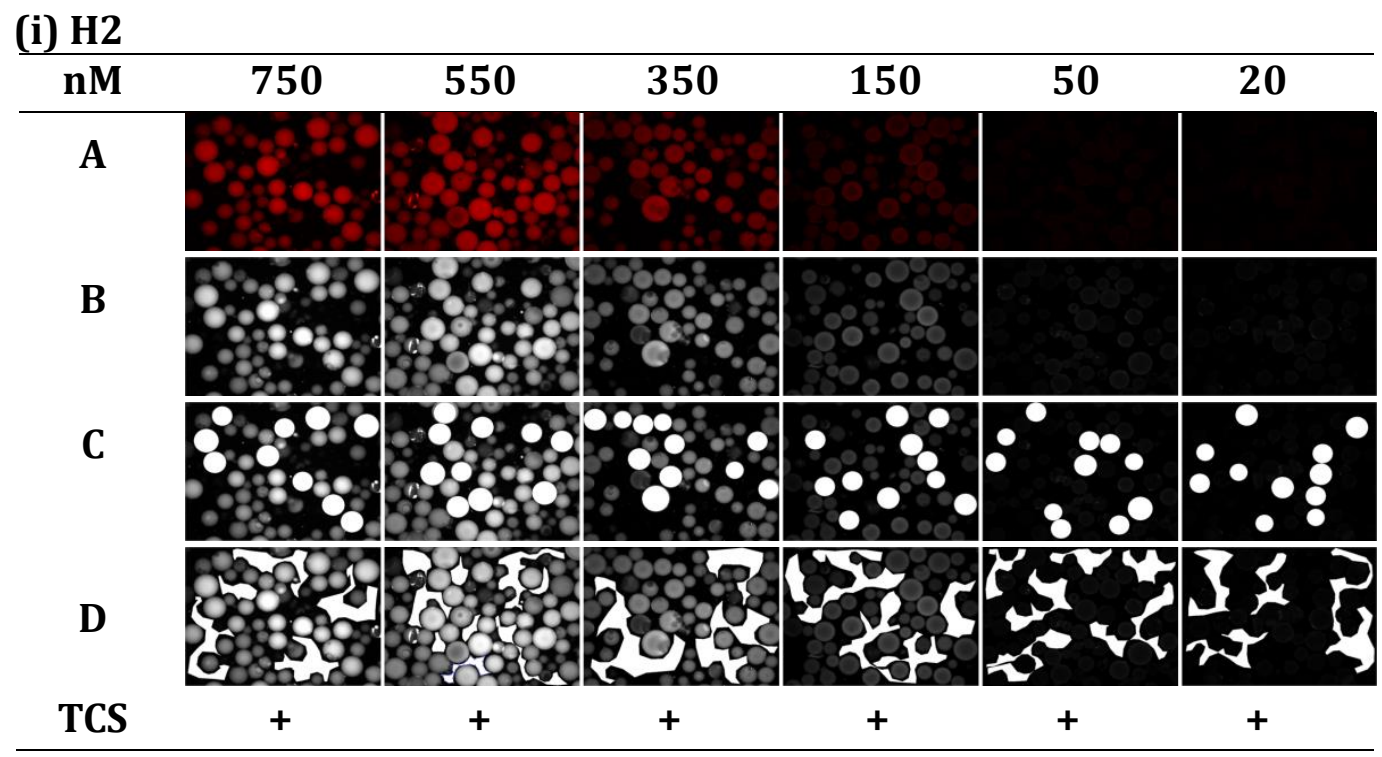

(ii) $\mathrm{H5}$

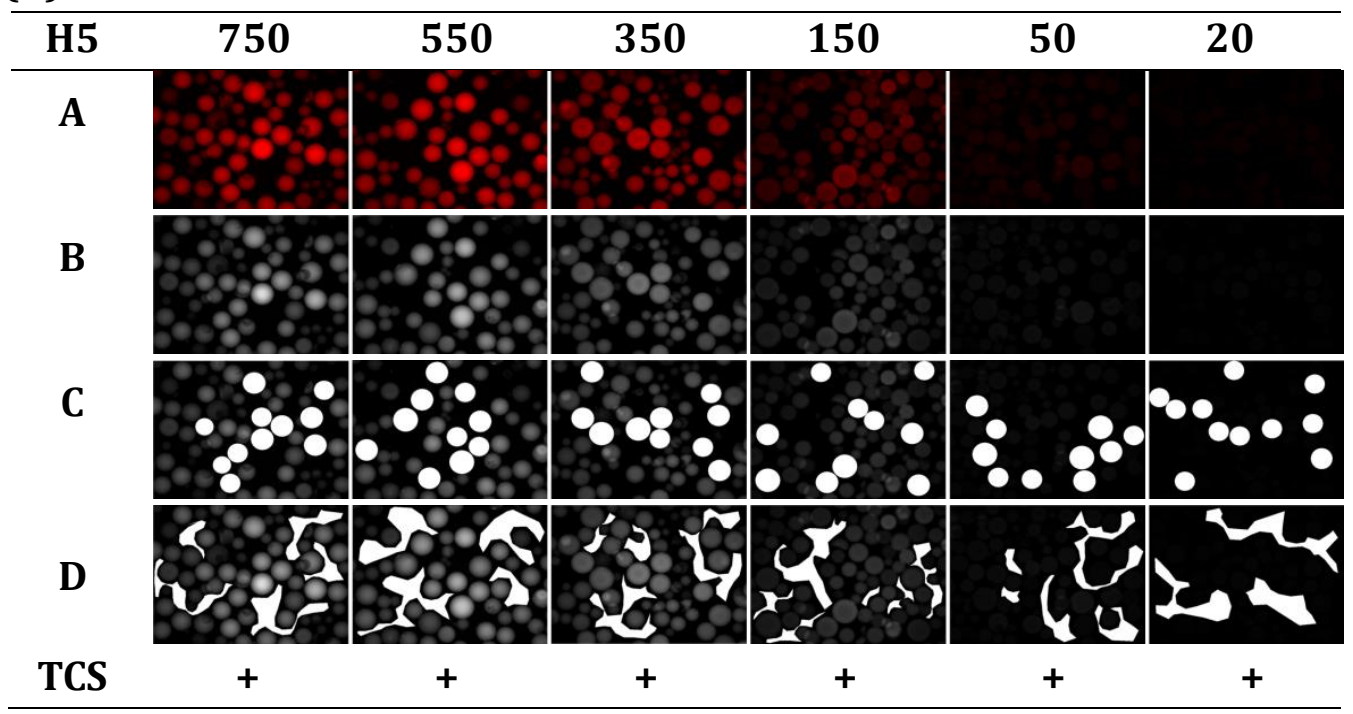




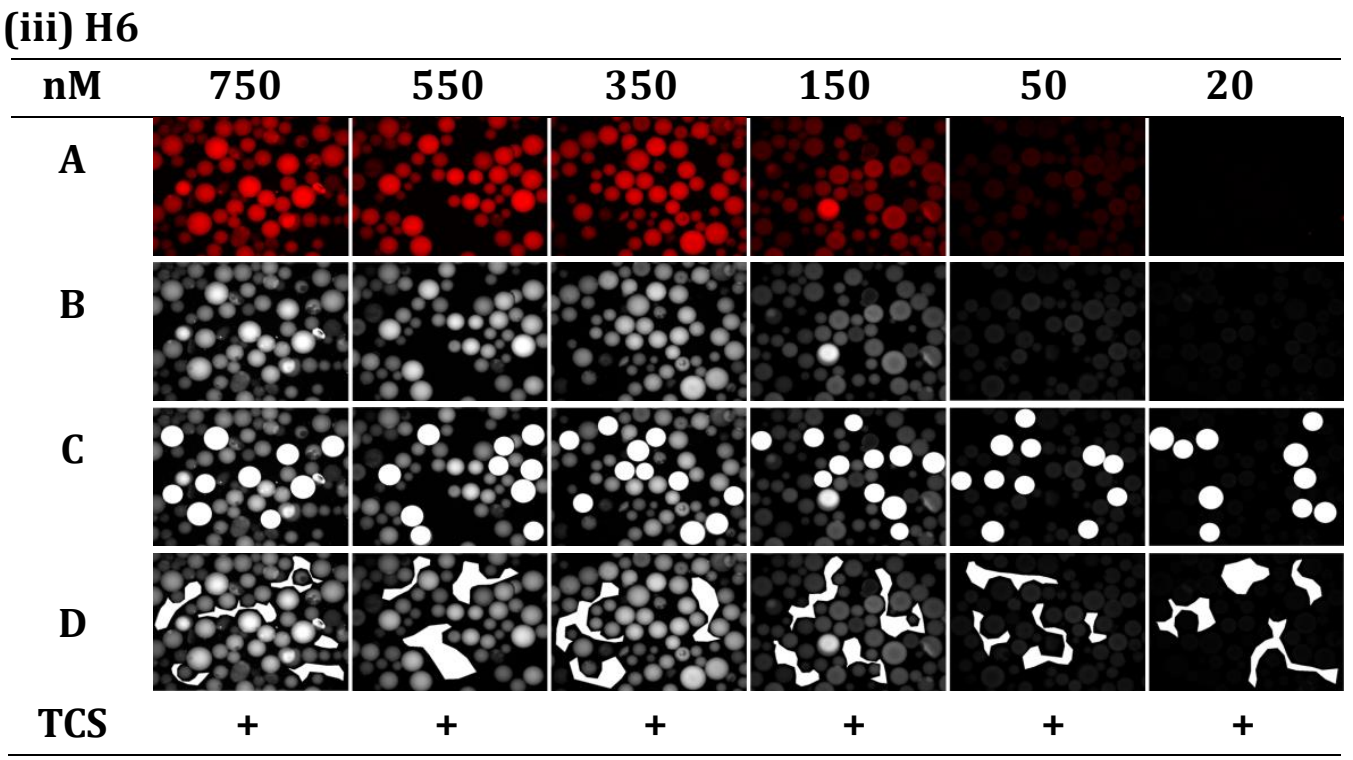

(iv) Rand

\begin{tabular}{|c|c|c|c|c|c|c|}
\hline $\mathbf{n M}$ & 750 & 550 & 350 & 150 & 50 & 20 \\
\hline A & & & & & & \\
\hline B & & & & & 9 & \\
\hline C & & & & & $0^{\circ}$ & \\
\hline D & & & & & & \\
\hline TCS & + & + & + & + & + & + \\
\hline
\end{tabular}

Each image was captured under the same conditions with 1 sec of exposure; "-" = control matrix and "+" = TCS-conjugated matrix. 


\section{APPENDIX VII}

Fluorescent images of the HEX-labelled aptamers (i) 57H6F, (ii) 58H6R and (iii) 40H6B binding to control matrix at $750 \mathrm{nM}$ and binding to triclosan (TCS) conjugated matrix at 6 concentrations ranging from $20 \mathrm{nM}$ to $750 \mathrm{nM}$. A to E represents 5 independent repeats of the same experiment.

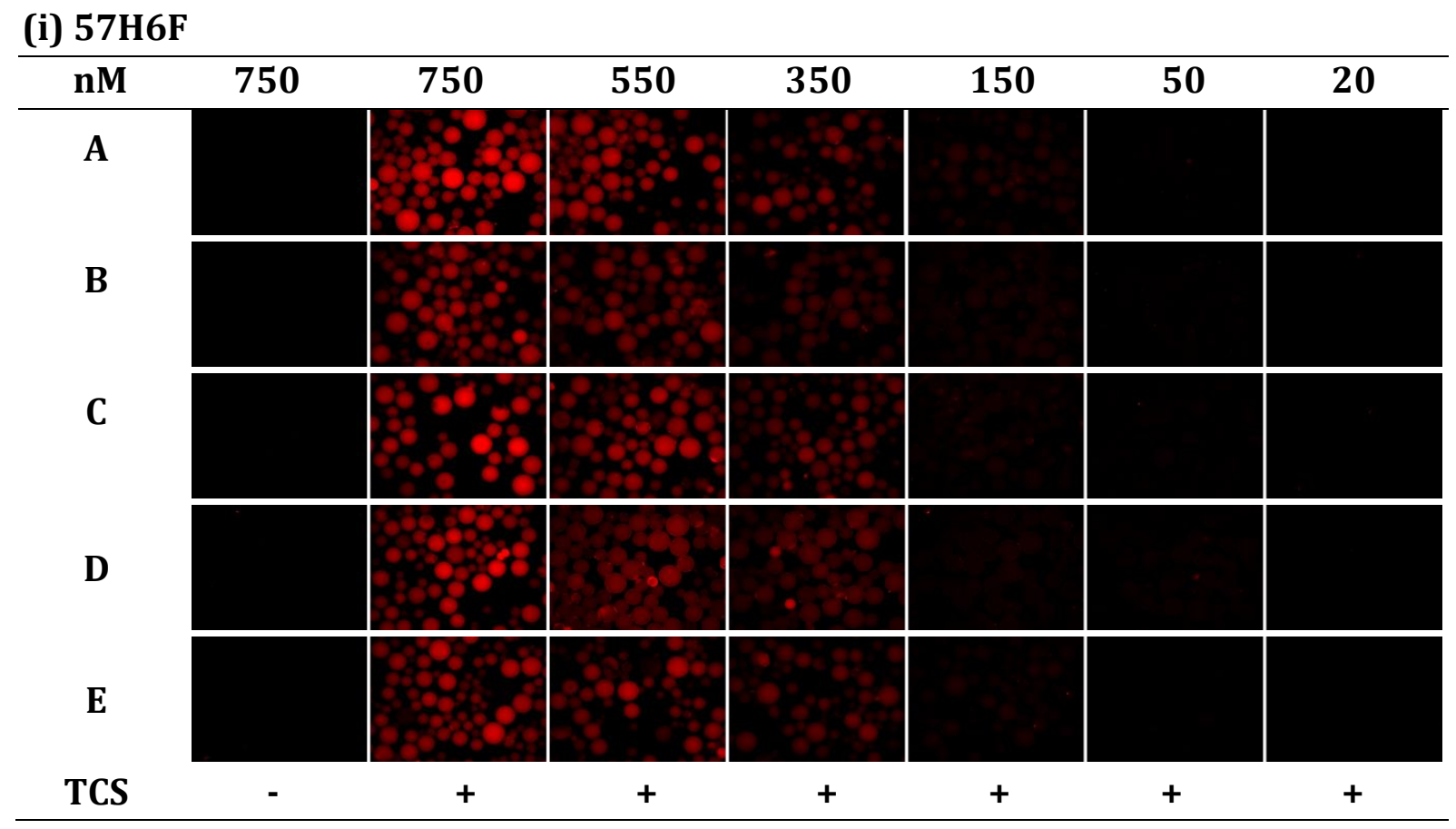

(ii) 58H6R

\begin{tabular}{|c|c|c|c|c|c|c|c|}
\hline $\mathbf{n M}$ & 750 & 750 & 550 & 350 & 150 & 50 & 20 \\
\hline A & & & & & & 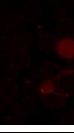 & \\
\hline B & & & & & & 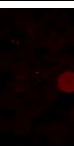 & \\
\hline C & & & & & & & \\
\hline D & & & & & & & \\
\hline $\mathbf{E}$ & & & & & 0 & & \\
\hline TCS & - & + & + & + & + & + & + \\
\hline
\end{tabular}


(iii) 40H6B

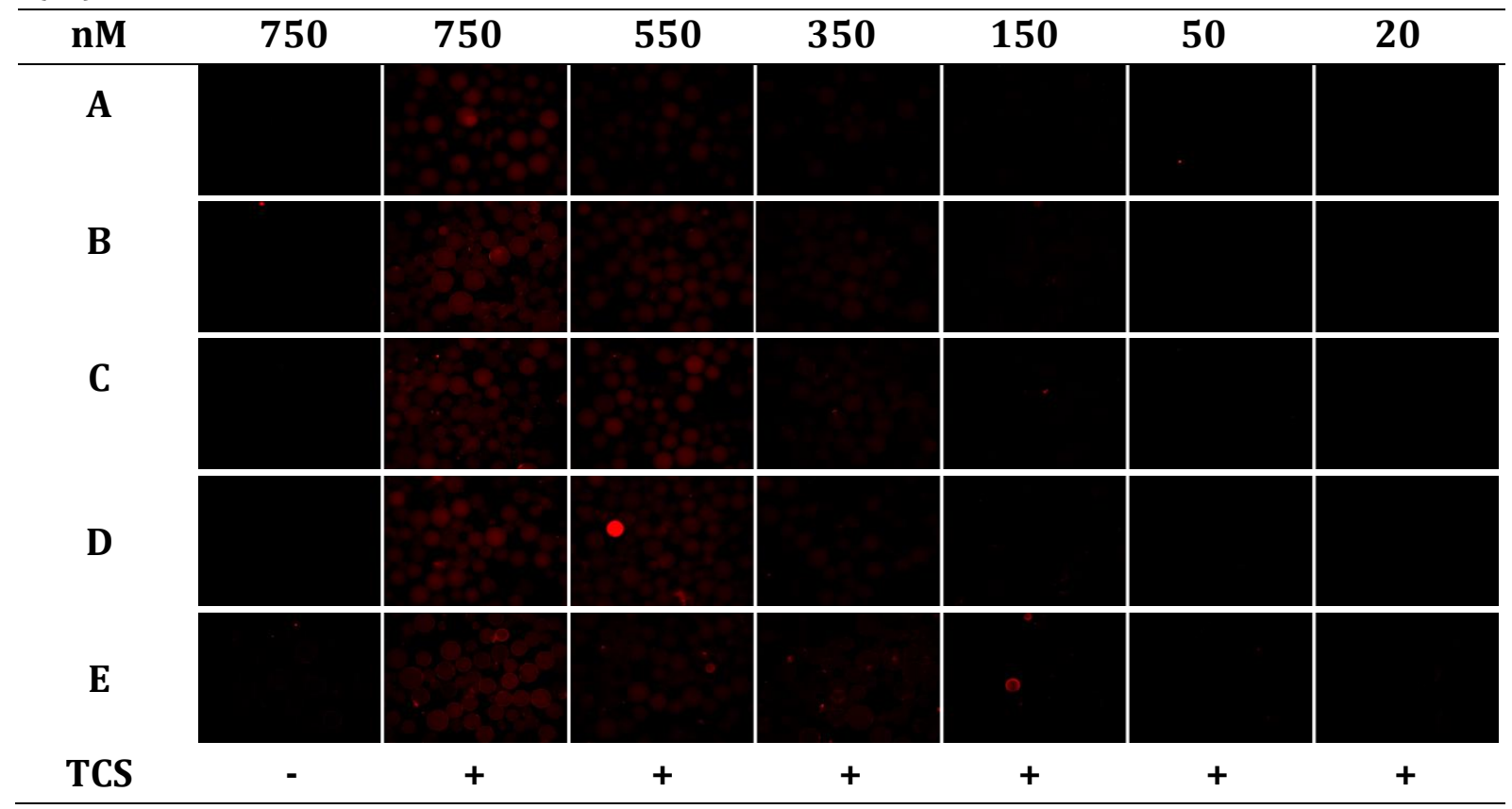

Each image was captured under the same conditions with 1 sec of exposure; “-” = control matrix and " + " = TCS-conjugated matrix. 


\section{APPENDIX VIII}

The following diagram demonstrats the results from the experiments using yeast tRNA for blocking both the control and TCS D matrices. An HEX-labelled random sequence (Rand) was chosen to assess the levels of nonspecific binding between the random sequence and the aforementioned matrices, and the concentration of tRNA used for blocking these matrices was varied from 5 to $20 \mathrm{mg} / \mathrm{mL}$.

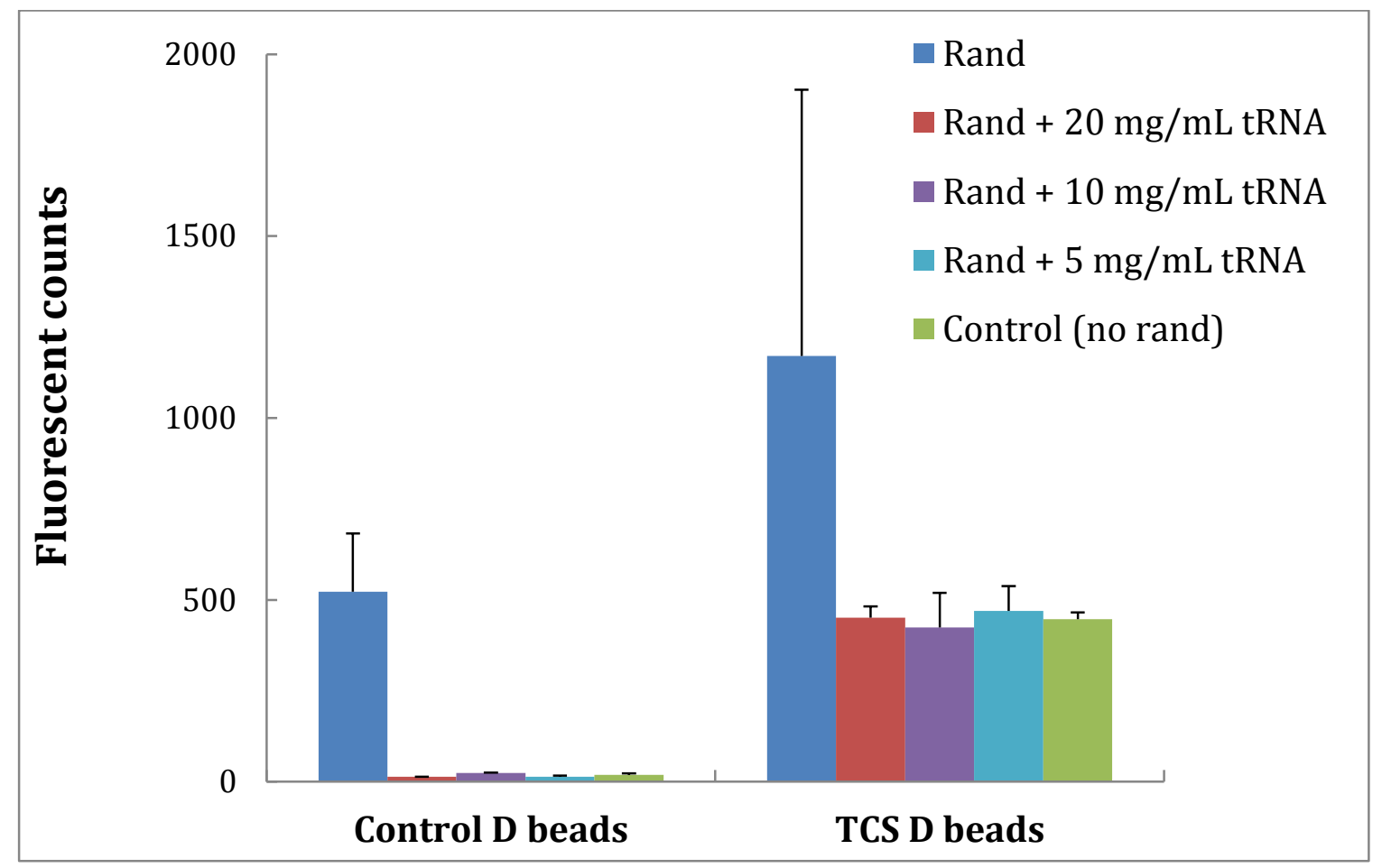

As shown in the diagram above, the random sequence showed considerable binding affinity to both the control and TCS D matrices without the presence of the tRNA. In contrast, only $5 \mathrm{mg} / \mathrm{mL}$ of the tRNA was required to completely restrict the binding of the random sequence to both matrices. As a precaution, $10 \mathrm{mg} / \mathrm{mL}$ of the tRNA was chosen in this study to block the affinity matrices used in the revised-SELEX procedure. 


\section{APENDIX IX}

Secondary structures of the H6 (TR10H6) and truncated H6 aptamers 57H6F, 58H6R and 40H6B showing minimal effect of truncation on the folding of the N40 region to form the stem-loop 3 (SL3).
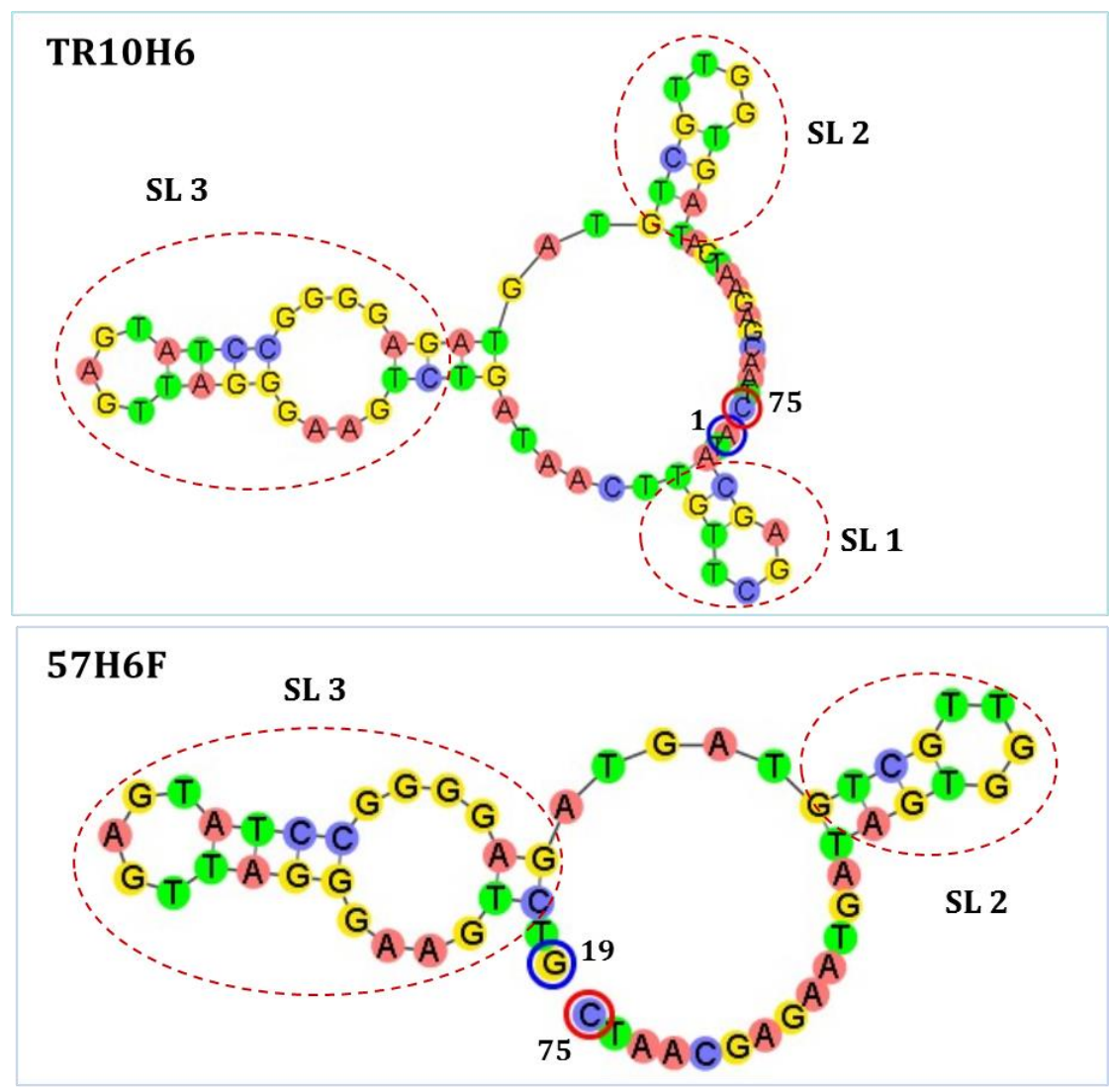

\section{H6B}

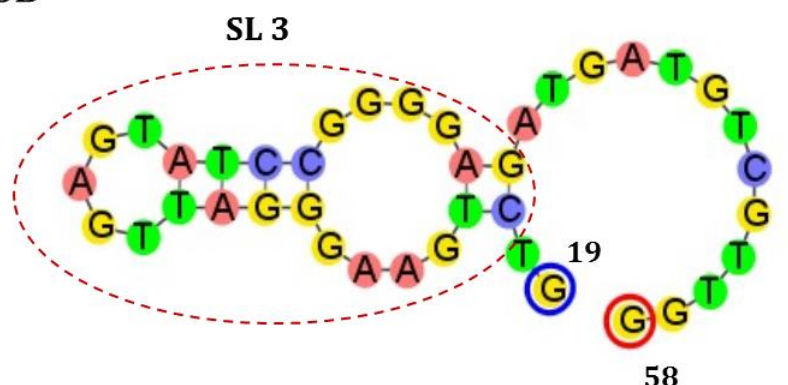

58

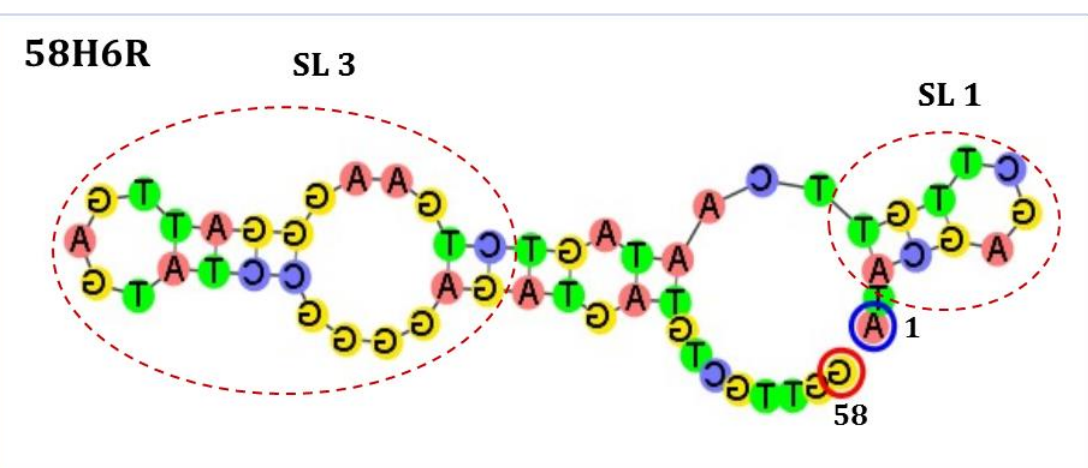

